SPECIES BOUNDARIES, REPRODUCTION AND

CONNECTIVITY PATTERNS FOR SYMPATRIC TETHYA

SPECIES ON NEW ZEALAND TEMPERATE REEFS

MEGAN RYAN SHAFFER

A thesis submitted to the Victoria University of Wellington In fulfilment of the requirements for the degree of Doctor of Philosophy

VICTORIA UNIVERSITY OF WELLINGTON

Te Whare Wānanga o te Ūpoko o te Ika a Māui 2019

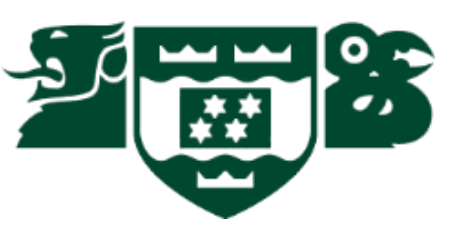



This thesis was conducted under the supervision of:

\author{
Professor James J. Bell \\ (Primary supervisor) \\ $\&$
}

Professor Simon K. Davy

(Co-supervisor)

Victoria University of Wellington

Wellington, New Zealand 



\begin{abstract}
Understanding the evolutionary forces that shape populations in the marine environment is critical for predicting population dynamics and dispersal patterns for marine organisms. For organisms with complex reproductive strategies, this remains a challenge. Sponges fulfil many functional roles and are important components of benthic environments in tropical, temperate and polar oceans. They have evolved diverse reproductive strategies, reproducing both sexually and asexually, and thus provide an opportunity to investigate complicated evolutionary questions. This $\mathrm{PhD}$ thesis examines sexual and asexual reproduction in two common golf-ball sponges in central New Zealand (Tethya bergquistae and T. burtoni), with particular focus on how the environment influeunces these modes of reproduction, and further, how they shape species delineations and connectivity patterns. New Zealand waters are projected to experience increases in temperature and decreases in nutrients over the next century, and therefore these species may be experience changes in basic organismal processes like reproduction due to climate change, requiring adaptation to local environments. Therefore, this work has important implications when considering how reproductive phenology, genetic diversity and population structure of marine populations may change with shifts in climate.
\end{abstract}

In my first data chapter, I highlight the difficulty in delineating sponge species by investigating the evolutionary relationship of Tethya spp. in central New Zealand using both morphological and molecular methods. Phylogenetic reconstructions based on two mitochondrial markers (rnl, COI-ext) and one nuclear marker (18S) revealed three genetic clades, with one clade representing $T$. bergquistae and two clades belonging to what was a priori thought to be a single species, T. burtoni. Morphological analysis based on spicule characteristics allowed $T$. bergquistae to be distinguished from $T$. burtoni, but revealed no apparent differences between the $T$. burtoni clades. These results indicate hidden genetic diversity within T. burtoni, which likely represents a group consisting of incipient species that have undergone speciation but have yet to express clear morphological differences. This chapter supports the notion that cryptic speciation in sponges may go undetected and diversity underestimated when using only morphology-based taxonomy, a result which has implications for conservation and management of marine systems. 
In my second data chapter, I characterize the reproductive biology for both species of Tethya in relation to potential environmental drivers, including sea surface temperature, chlorophyll$a$ concentration and rainfall. Using histological methods for sponges collected monthly over two years, Tethya spp. were found to be gonochoristic and oviparous sexual reproducers, with one annual reproductive event occurring in the austral summer from January to March. Differences in oocyte density and reproductive output between both species and sites highlighted both species-specific adaptive responses and environmental influences on reproduction. Temperature and rainfall were found to be correlated with instances of sexual reproduction, and the summer reproductive event occurred each year following the spring bloom of chlorophyll- $a$. These findings indicate that seasonal fluctuations in the environment may be important for triggering gametogenesis for these species. With shifts in temperature, productivity, and timing of seasons projected for New Zealand, there is a potential for reproductive phenology to become mismatched with the surrounding environment under future climate change scenarios, which has consequences for the frequency, duration and overall output of sexual reproduction for these sponges.

My third data chapter characterizes asexual reproduction in both species of Tethya, exploring relationships between reproductive traits and potential environmental drivers that may influence asexual budding events. Two sponge populations, one for each species of Tethya, were monitored over two years by both monthly sampling and periodic in situ observations. Data revealed that budding occurred continuously throughout the year, but had a cyclic pattern where instances of budding and densities of buds were higher during the austral spring and summer. Asexual reproduction coincided with sexual reproduction, and some individuals were found to simultaneously reproduce using both modes. Instances of asexual reproduction were positively associated with temperature and rainfall, but distinct differences between species were difficult to identify. As temperature proved important, an experiment looking at bud production in relation to thermal stress was conducted, where sponges were subjected to stable temperatures treatments of $17^{\circ} \mathrm{C}$ (control), $19^{\circ} \mathrm{C}$ and $21^{\circ} \mathrm{C}$. No instances of budding were observed under any temperature treatment, and high mortality occurred in the $21^{\circ} \mathrm{C}$ treatment. These results suggest that temperature changes (i.e., heterogeneous environments) may be more important than temperature alone in driving asexual reproduction, and further, indicate thermal stress will result in increased sponge mortality. Correlations to potential environmental drivers indicate that future shifts in climate may affect instances of asexual reproduction and 
thus sponge abundance, which has the potential to alter the genetic structure and overall diversity of these populations.

In the final data chapter, I developed novel microsatellite markers for Tethya burtoni to characterize the genetic connectivity patterns among four populations in central New Zealand, with particular interest in the roles that sexual and asexual reproduction play in connectivity. I sampled three sites within $10 \mathrm{~km}$ of each other in the Wellington Region (WR), and another site on an island (Kapiti Island) approximately $50 \mathrm{~km}$ north of the WR. At one of the WR sample sites, I monitored a T. burtoni population over two years to examine the dispersal range of asexually reproduced buds and the ability of clones to sexually reproduce. The WR and Kapiti Island populations were strongly genetically differentiated, but within the WR region, two populations were genetically similar, indicative of high connectivity. For the monitored population, asexual bud dispersal was restricted to no greater than $1 \mathrm{~m}$ and clonal individuals had reduced sexual reproductive ability. Asexual reproduction did not appear to play an important role in interpopulation connectivity nor gene flow, as buds had low dispersal ability and rarely reproduced. Population structure and connectivity for T. burtoni appear to be largely driven by sexual reproduction, and asexual reproduction instead aids genotype survivorship and population maintenance. These findings highlight that different reproductive modes can differentially contribute to population dynamics in sessile marine organisms, suggesting that predictions about future population viability under changing environments may be difficult to make.

In summary, this $\mathrm{PhD}$ thesis uses a combination of genetic, histological, field-based and experimental methods to examine species boundaries, reproduction and connectivity for Tethya spp. on rocky reefs of New Zealand. The sympatric nature, complex reproductive ecology and connectivity patterns observed likely shape the complex evolutionary processes occurring in these sponges, including introgressive hybridization and cryptic species. Individuals that showed evidence of possible introgressive events occurred mainly in populations with more restricted gene flow, while the presence of both cryptic species were more prevalent in well connected populations. Such a trend allows for discussion of under what circumstances both of these processes occur. Furthermore, environmental correlates to both sexual and asexual reproduction indicate that both of these modes of reproduction have the potential to be altered 
with future changes in the environment. As both modes were found to play different roles in gene flow within and between populations, future shifts in climate are also expected to alter population structure and connectivity for these sponges. Such shifts in gene flow will also likely result in changes to species boundaries and thus the overall diversity of this genus. Many other sessile, benthic marine organisms present reproductive traits and behaviours similar to those of Tethya spp., and therefore these results can aid in the interpretation of results for other marine taxa. Overall, this thesis describes the population dynamics of Tethya spp., which are abundant and ecologically important on New Zealand reefs, and provides insight on how temperate sponge populations may fare with climate change, which has important implications for management and conservation efforts. 
This thesis is dedicated to my loving and supportive parents, Jim and Peggy Shaffer. 



\section{ACKNOWLEDGEMENTS}

To begin, I firstly need to thank James for his excellent support and supervision throughout my thesis. I was privileged to be supervised by a perceptive, hard-working and tenacious scientist, and am grateful to have had such an impressive role model during my $\mathrm{PhD}$. I particularly appreciate the tremendous opportunities that James provided me, and am fortunate that my thesis journey is one that goes well beyond what is encompassed in this document. I owe another thanks to my co-supervisor, Simon, who offered invaluable insight and constructive comments while reading my manuscripts and chapters. Lastly, a special thanks to Manuel Maldonado (Centre for Advanced Studies of Blanes, CEAB-CSIC), who took the time to thoughtfully review my histological images and genetic data, and to assist in the interpretation of my sometimes confusing results.

I am grateful for the contributions made by many people to various chapters of my thesis. For Chapter 2, a special thanks to Michael Gardner and Tessa Bradford (Flinders University) for their help with microsatellite development, and to Daniel Leduc (National Institute of Water and Atmospheric Research) for training and use of the SEM. I am also grateful to Matt Pinkerton (National Institute of Water and Atmospheric Research), who provided time series satellite data for sea surface temperature and chlorophyll- $a$ concentration used in both Chapters 3 and 4. A thanks to Lisa Woods (Victoria University of Wellington) for her assistance with statistical analyses for Chapters 3 and 4, and to Balam for guidance with genetic analyses in Chapters 1 and 2. Lastly, a whole-hearted thanks to the technicians at the Victoria University Coastal Ecology Lab (Daniel 'Snout' McNaughtan, John van der Sman, Dan Crossett), skipper Don Nelson, and to Becky and Albi, who were integral in assisting with field and lab work for all chapters of this thesis. A further thanks to dive buddies: Tracey, Cliff, Emily, Andrea and Phoebe. Lastly, I would like to acknowledge Victoria University for providing me funding (Doctoral and FSRG Scholarships) that supported this research and allowed for attendance of conferences and presentation of this work.

I was lucky to work in such a collaborative and enthusiastic lab group, and owe a thanks to members (new and old) of the Bell Lab, or 'Sponge Club'. A special thanks to Joe and Emily for dragging me to Hoga and showing me the creative side of fieldwork. And to Emily 
especially, a thanks for the constant encouragement and cathartic chats throughout my $\mathrm{PhD}$. I was also privileged to be able to work in a beautiful work space (above and below water) thanks to VUCEL, and owe a special thanks to the VUCEL community for their constant support over the past years. In particular, I would like to acknowledge fellow VUCEL-ers Bucky and Snout, who made this $\mathrm{PhD}$ experience a fun one. To Bucky, a friend I was lucky enough to have alongside me (quite literally) throughout this entire journey, I am so grateful for both the time you gave to all of my field/lab work and the time you gave to our day-long desk cackles, which kept me sane throughout this process (at the expense of everyone else in our office). A special thanks to you (and Mike) for picking me up (again, quite literally) during the end. To Snout, a thanks for the invaluable dive training provided over the past four years and a thanks for putting up with Bucky's and my shenanigans.

Lastly, I would like to thank my family and friends, for without their love and encouragement, this $\mathrm{PhD}$ thesis would not have been at all possible. To my parents, who let me put this thesis before all other things multiple times, and who have always supported and fostered my love for this field from early days: the work put into this $\mathrm{PhD}$ thesis pales in comparison to the hard work and sacrifices you've made that have allowed me to travel across the world and pursue this career. I could never have done this without you, and I could never thank you enough. To Jessica (+ Odie), Kelsey, Christian, Nick - the five that I've missed the most these past years: thanks for cheering me on, inspiring me and always lending an ear, especially during the tougher bits at the end. And finally, to my partner Albi, the best thing to come from this adventure of a $\mathrm{PhD}$ : I am beyond grateful for the constant love and support during this process, and thank you for keeping me motivated, keeping me balanced, and most importantly, keeping me laughing. 


\section{TABLE OF CONTENTS}

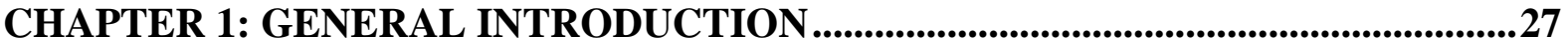

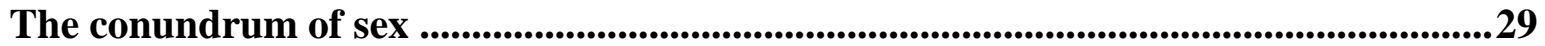

Connectivity and species boundaries in the marine realm .............................................31

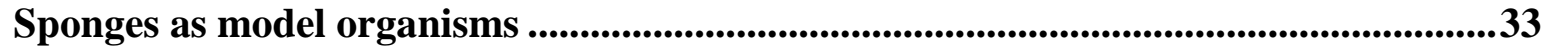

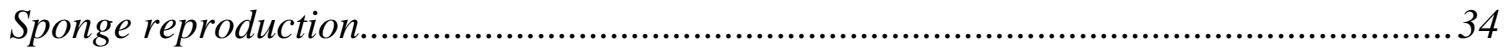

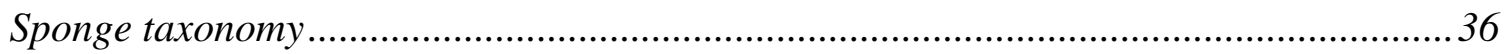

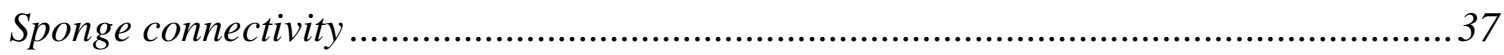

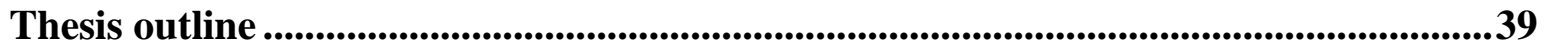

CHAPTER 2: HIDDEN DIVERSITY IN THE GENUS TETHYA: COMPARING MOLECULAR AND MORPHOLOGICAL TECHNIQUES FOR SPECIES IDENTIFICATION .........................................................................................................................4

Introduction ....................................................................................................................................... 43

Materials and Methods .............................................................................................................................45

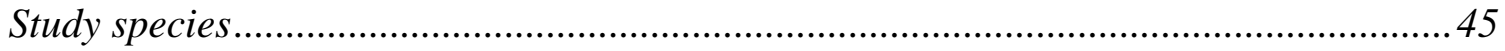

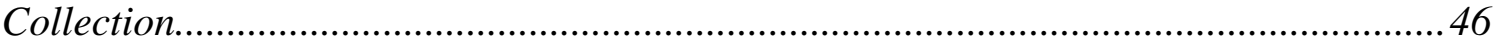

DNA extraction, PCR amplification, and sequencing ….............................................. 48

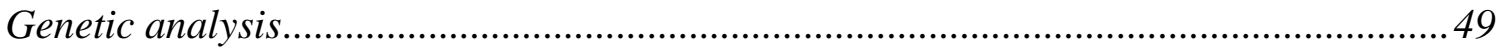

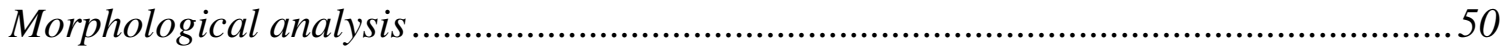

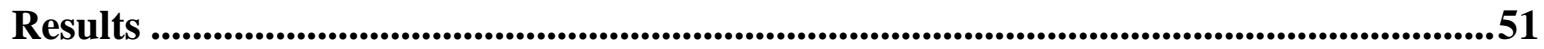

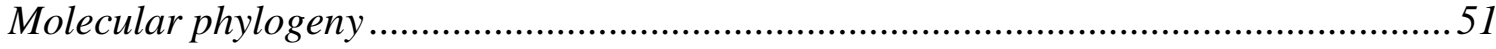

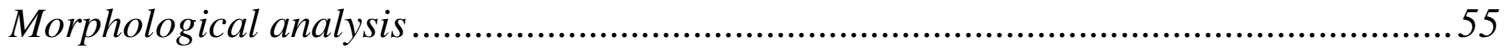

Discussion ....................................................................................................................................59

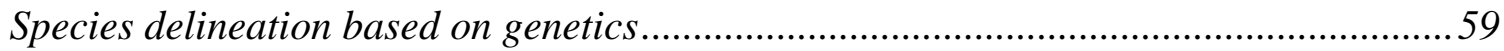

Discordance between molecular and morphological characteristics ............................ 61

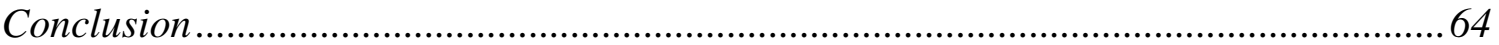

CHAPTER 3: SEASONAL SEXUAL REPRODUCTION IN TETHYA SPP. ON NEW ZEALAND TEMPERATE REEFS................................................................................65

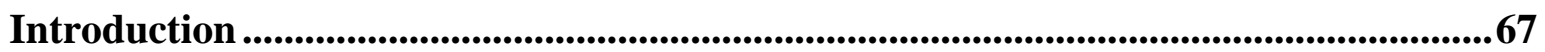

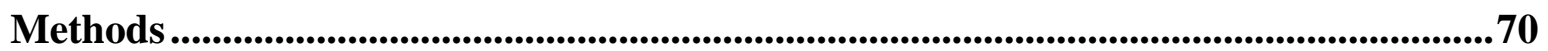

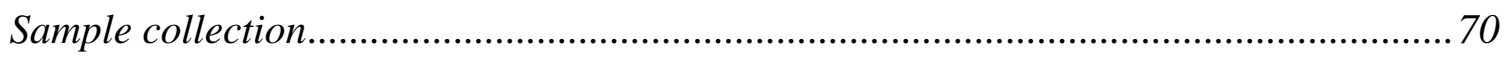

Histological processing and quantification of reproductive propagules .......................... 70

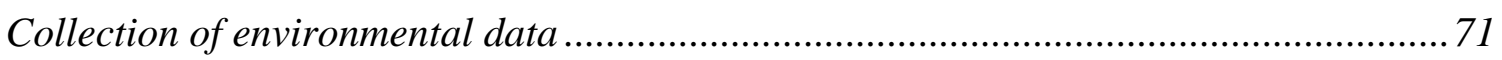

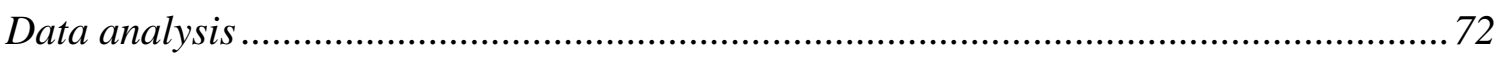

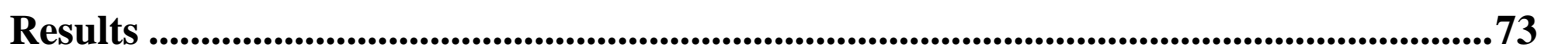




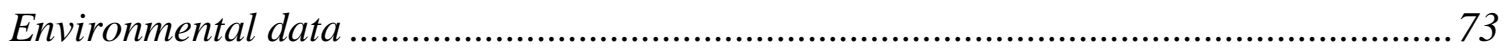

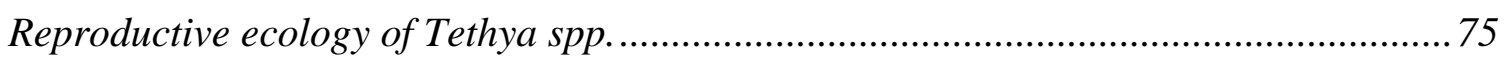

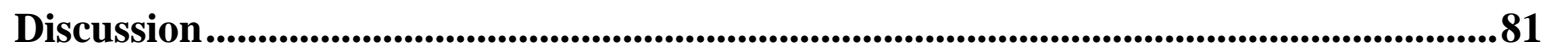

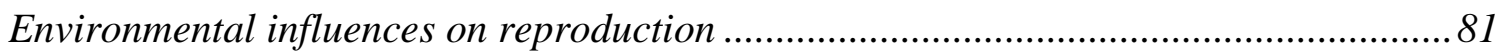

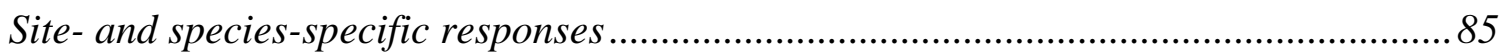

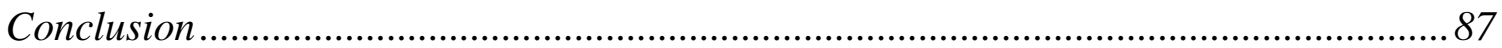

CHAPTER 4: POTENTIAL ENVIRONMENTAL DRIVERS OF ASEXUAL

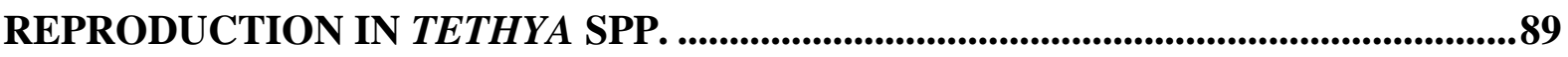

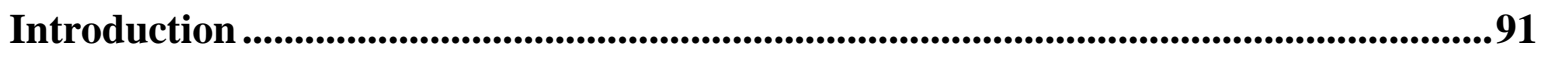

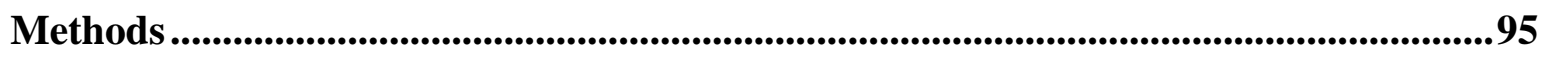

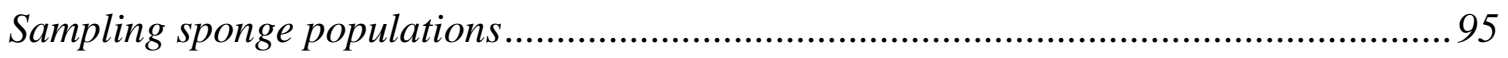

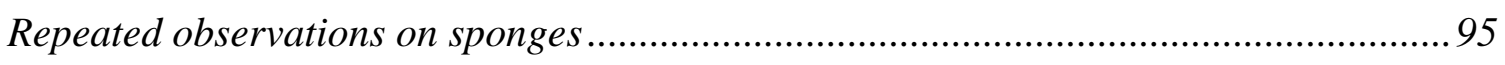

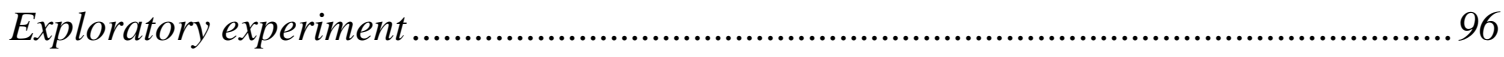

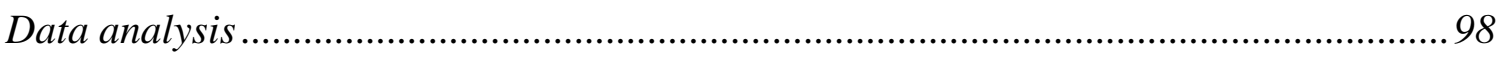

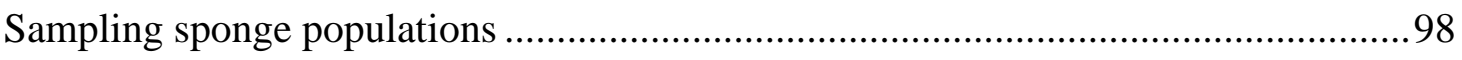

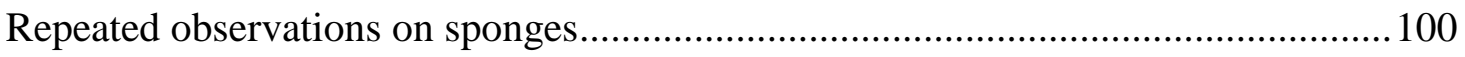

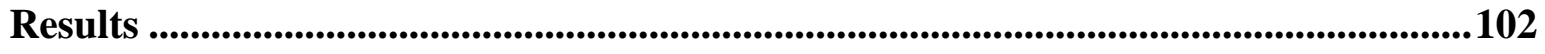

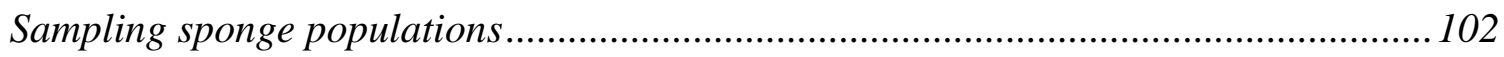

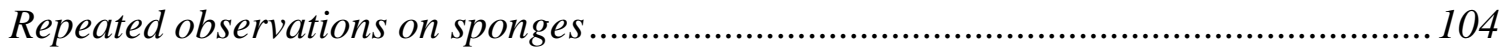

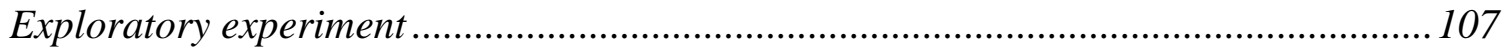

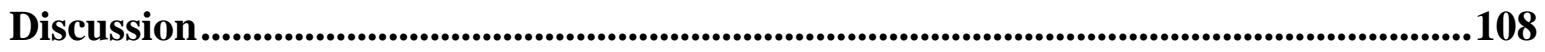

Environmental influences on asexual reproduction ................................................... 109

Differences between species: adaptive significance ................................................... 111

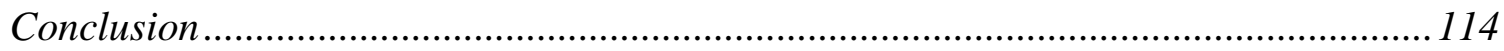

CHAPTER 5: DIFFERENTIAL ROLES OF ASEXUAL AND SEXUAL REPRODUCTION IN GENOTYPE SURVIVORSHIP AND CONNECTIVITY IN THE NEW ZEALAND SPONGE TETHYA BURTONI ................................................... 115

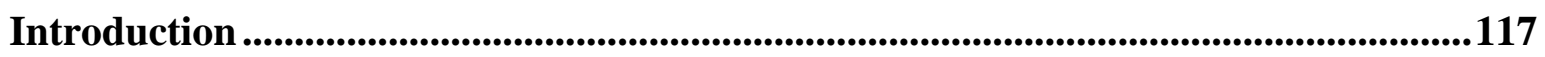

Materials and methods......................................................................................................120

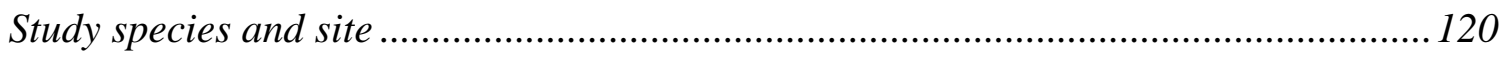

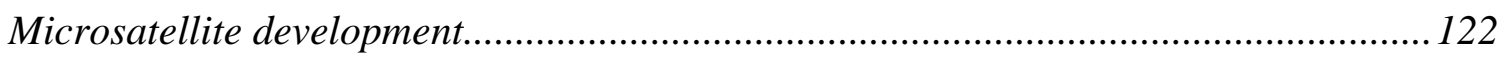

Sample collection, DNA extraction and PCR amplification......................................... 123

Population genetic analyses: basic genetic diversity indices........................................ 124

Population genetic analyses: population differentiation............................................ 125

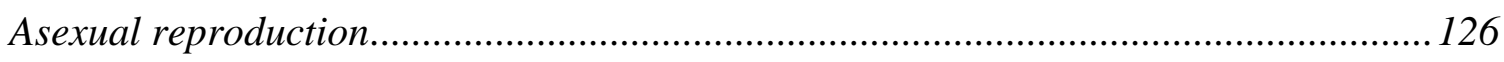

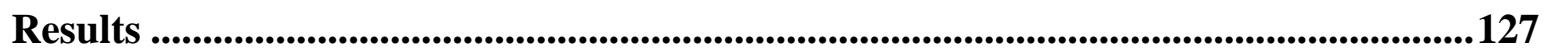


Genetic diversity indices.

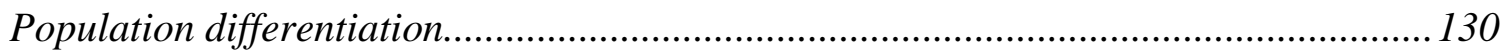

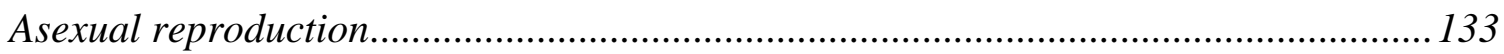

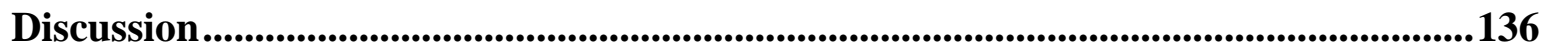

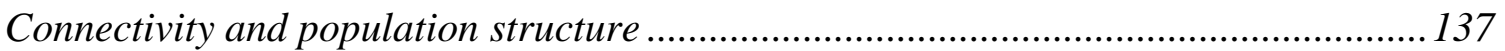

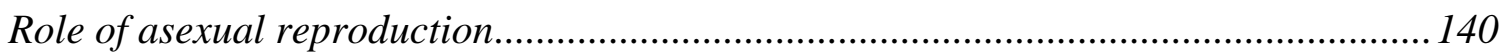

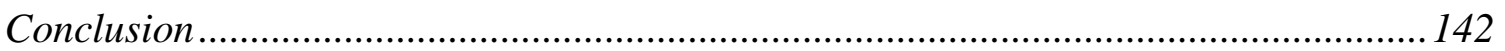

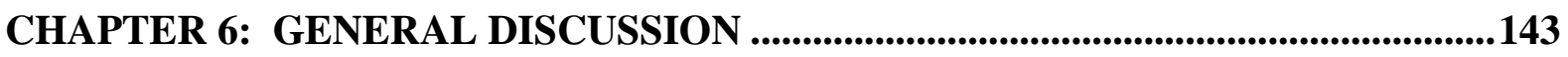

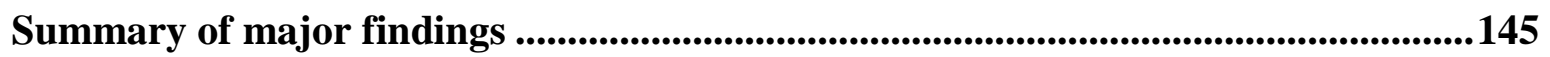

Comparing methods for species delineations.......................................................................146

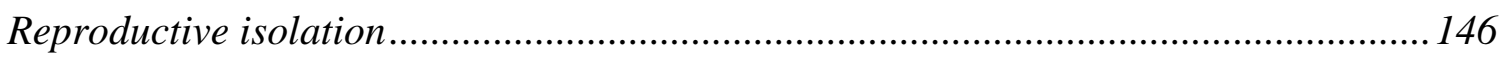

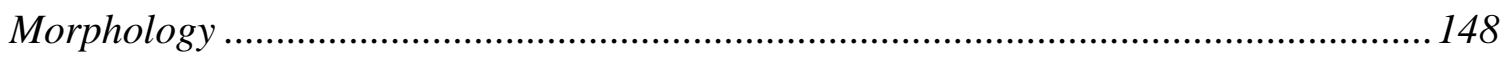

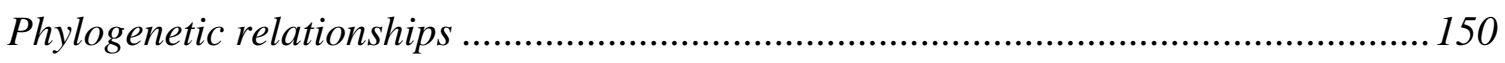

Evolutionary forces shaping Tethya populations: connectivity, cryptic speciation and introgression ............................................................................................................................152

Species-specific responses ..................................................................................................156

Comparison of sexual reproduction in Tethya versus other sponges from the temperate

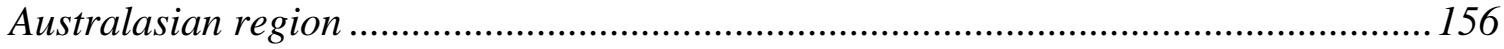

Tethya bergquistae as a more thermally tolerant sponge than T. burtoni .................... 159

Changes in population dynamics as a result of climate change .....................................161

Future directions ...............................................................................................................164

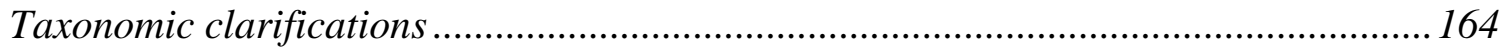

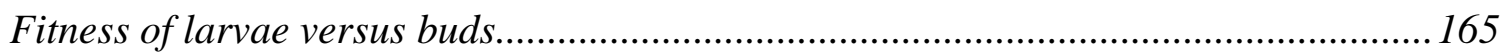

Parentage analyses, hybridization, introgression and chimerism: microsatellites as a

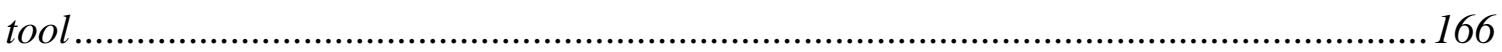

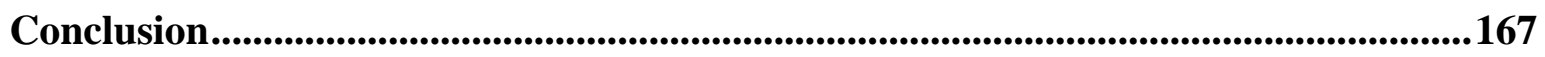

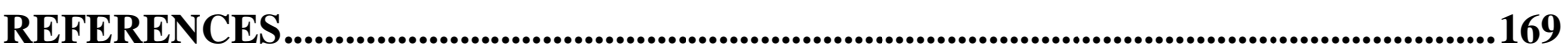

APPENDIX 1: INFORMATION FOR SAMPLES USED IN PHYLOGENETIC ANALYSES ............................................................................................................................2203

APPENDIX 2: DEFINITION OF SPICULE TYPES ........................................................207

APPENDIX 3: CORRELATIONS WITH ENVIRONMENTAL COVARIATES........209

APPENDIX 4: DIFFERENCES IN ROI BETWEEN SITE AND SPECIES.................211

APPENDIX 5: SUMMARIES OF RESULTS FROM ANOVAS AND LR $\chi^{2}$ TESTS FROM CHAPTERS 3 AND 4

APPENDIX 6: IN SITU OBSERVATIONS OF BUDDING EVENTS RECORDED FOR MONITORED SPONGES .........................................................................................................217 
APPENDIX 7: INFORMATION ON CLONAL GROUPS.

.225

APPENDIX 8: LINKAGE DISEQUILIBRIUM BETWEEN PAIRS OF MICROSATELLITE LOCI ................................................................................227

APPENDIX 9: PUBLICATION OF CHAPTER 2............................................229 


\section{LIST OF FIGURES}

Figure 1.1 Regeneration of sponges used in this thesis over a two-year study period

Figure 2.1. Examples of sponges collected belonging to clades identified in Figure 3, to allow comparison of macromorphological features. For each clade, cross sections that encompass the ectosome and choanosome are included for a subsample $(n=3)$ of genetically identical individuals. Clade 1 (top) are examples of Tethya bergquistae, while Clades 2 and 3 (middle and bottom, respectively) are examples of $T$. burtoni. Scale bars in each photo $=10 \mathrm{~mm} . . . .47$

Figure 2.2. Phylograms showing the relationship between Tethya bergquistae (Clade 1) and T. burtoni (Clades 2 and 3) based on individual mitochondrial markers (rnl and COI-ext). Topology generated from Maximum Likelihood (ML) analysis. ML bootstrap confidence values/Bayesian Inference (BI) posterior probabilities given on each branch. Tethya actinia was selected as an outgroup. Inconsistencies in the COI-ext tree versus rnl are highlighted by having sponges retain the colour of the rnl placement and by bolding individuals. Scale bar = substitutions per site.

Figure 2.3. Phylograms showing the relationship between Tethya bergquistae (Clade 1) and T. burtoni (Clades $2 \mathrm{~A}+\mathrm{B}$ and 3) based on nuclear DNA (18S) and mitochondrial DNA (concatenated COI-ext $+\mathrm{rnl}$ ). Topology generated from Maximum Likelihood (ML) analysis. ML bootstrap confidence values/Bayesian Inference (BI) posterior probabilities given on each branch. T. actinia was selected as an outgroup. Scale bar $=$ substitutions per site.

Figure 2.4. Nonparametric-multidimensional (nMDS) scaling ordination of spicule sizes for Tethya bergquistae (pink squares, Clade 1) and T. burtoni (orange circles, Clade 2; and blue triangles, Clade 3). Clades are identified in Figures 3. Spicules sizes are based on measurements from strongyloxea, megasters and micrasters $(n=30$ per sponge $)$. Codes above each sample refer to individual sample ID (refer to Appendix 1 for more information on individual samples). A subset of all samples is presented in the nMDS, based on a Euclidean distance matrix.....55

Figure 2.5. Scanning electron micrographs of spicules belonging to Tethya bergquistae (Clade 1) and Tethya burtoni (Clade $2+3$ ), from sponges belonging to the three genetic clades of Tethya identified in Figure 3. Clade 1: A-C = spherasters; $\mathrm{D}=$ spined oxyaster; $\mathrm{E}-\mathrm{H}=$ tylasters; $\mathrm{G}=$ oxyaster. Clade 2 and $3: \mathrm{A}-\mathrm{B}=$ spherasters $\mathrm{C}-\mathrm{D}=$ tylasters $; \mathrm{E}=$ oxyaster. Scale bars are located in the bottom right of each micrograph and represent $10 \mu \mathrm{m}$. .57

Figure 2.6. Cross sections from small pieces of tissue (20 $\mu \mathrm{m}$ thick) from Tethya spp., where Clade 1 is T. bergquistae and Clades 2 and 3 are T. burtoni. Arrows are pointing to the line that divides the outer cortex and the inner choanosomal tissue. Black scale bars represent $500 \mu \mathrm{m}$.

Figure 3.1. Environmental data from the Wellington south coast from January 2015 to February 2017. Monthly means given for sea surface temperature (SST, in ${ }^{\circ} \mathrm{C} ; \mathrm{A}$ ) and chlorophyll- $a$ 
concentration (chl- $a$, in $\mathrm{mg} \cdot \mathrm{m}^{-3} ; \mathrm{B}$ ), whereas monthly rainfall (in $\mathrm{mm}$; C) corresponds to total monthly volumes.

Figure 3.2. Examples of tissue sections containing gametes (oocytes, o; and spermatic cysts, sc). For oocytes, tissues of both Tethya burtoni (A) and T. bergquistae (B) are shown. Spermatic cysts in tissue belonging to T. burtoni (C) .75

Figure 3.3. Monthly proportion of sexually reproductive sponges from Feb 2015-2017 for Tethya bergquistae (black) and T. burtoni (grey). Monthly means for sea surface temperature $\left(\mathrm{SST},{ }^{\circ} \mathrm{C}\right)$ overlaid on top graph and total monthly rainfall $(\mathrm{mm})$ on bottom.

Figure 3.4. Environmental effects on the probability of sexual reproduction by Tethya spp. Environmental effects include: sea surface temperature (A), rainfall (B) and chlorophyll- $a$ concentration (C). Bottom ticks across the $\mathrm{x}$-axes represent an observed response from a sponge, and shaded area around lines represent $95 \%$ confidence intervals.

Figure 3.5. The effects of site (top) and species (bottom) on oocyte density for Tethya bergquistae (purple) and T. burtoni (green) from both Breaker Bay (blue) and Princess Bay (orange). Plots on left are means of the $\log$ (oocyte density) between site (A) and species (C), and bars are $95 \%$ confidence intervals. Plots of right are differences in oocyte density between sites (B) and species (D) presented by month, where the entire reproductive event captured is highlighted in grey. Sample sizes for species collected from each site are presented in Table 3.1. Error bars on plots on right correspond to standard error. 78

Figure 3.6. Monthly average oocyte sizes (surface area, SA; in square microns) for both Tethya bergquistae and $T$. burtoni across all sites. The entire reproductive event captured is highlighted in grey, and error bars on plots on right correspond to standard error.

Figure 3.7. Box plot representing differences in sponge size (diameter, $\mathrm{mm}$ ) between Tethya bergquistae (purple) and T. burtoni (orange) from two sites

Figure 3.8. The effect of sponge size (diameter, in $\mathrm{mm}$ ) on the probability of sexually reproducing (A), oocyte density (B), oocyte size (C) and ROI (D) for Tethya bergquistae and T. burtoni. ROI, or the reproductive output index, is the percent of tissue occupied by gametes. Bottom ticks across the $\mathrm{x}$-axes represent an observed response from a sponge, and shaded area around lines represent $95 \%$ confidence intervals.

Figure 4.1. Examples of budding in Tethya bergquistae (A) and T. burtoni (B). .95

Figure 4.2. Temperature readings from deployed temperature logger at Breaker Bay from October 2015-May 2018 (A), with highlighted area enlarged in plot B. Grey bars on plot B highlight a change in temperature greater than $1^{\circ} \mathrm{C}$ experienced within $24 \mathrm{~h}$. 
Figure 4.3. Differences in the probability of budding for Tethya bergquistae (purple) and $T$. burtoni (green) at different sites, where error bars represent $95 \%$ confidence intervals

Figure 4.4. The effect of sponge size (A) and chlorophyll-a concentrations (B) on Tethya bergquistae and T. burtoni. Shaded regions represent $95 \%$ confidence intervals.

Figure 4.5. Budding events in relation to sea surface temperature (SST). Top plot presents the proportion of budding sponges collected each month, whereas bottom plot shows the effect of SST with increasing concentrations of chlorophyll- $a$ (chl- $a$ ) for both species pooled (as there was no significant interaction between species $\times$ chl-a $\times$ SST). Shaded regions on chl- $a$ plots represent $95 \%$ confidence interval

Figure 4.6. Bud density in relation to sea surface temperature $(A, B)$ and rainfall $(C, D)$. Monthly means of bud density for each species given (B, D), with error bars representing standard error. Shaded regions surrounding regression lines (A, C) represent $95 \%$ confidence intervals 106

Figure 4.7. Bud density in relation to sponge size for both species, where shaded regions represent $95 \%$ confidence intervals. .107

Figure 4.8. Sea surface temperature readings versus temperature readings from deployed HOBO logger. .108

Figure 4.9. Fusion event between two Tethya bergquistae. 108

Figure 5.1. Tethya burtoni (top sponge) asexually reproducing, with buds (B) labelled. .....121

Figure 5.2. Map of study sites in central New Zealand used for this PhD thesis. Sites for population structure (Chapter 5) are indicated by coloured circles, and include Kapiti Island (KP), and three sites within the Wellington region (WR): Breaker Bay (BB), the South Coast (SC), and Matiu-Somes Island (SI). Red Rocks (sampled in Chapter 2) is the same site SC. Princess Bay (PB, sampled in Chapters 3 and 4) indicated by an X. Scale bars given in the bottom right.

Figure 5.3. Isolation by distance for populations of Tethya burtoni, where pairwise $F_{\mathrm{ST}} /(1-$ $\left.F_{\mathrm{ST}}\right)$ between populations is plotted against the logarithm of geographical distances $(\mathrm{km})$ between populations. P-value determined by a Mantel test

Figure 5.4. Population differentiation between four populations (Breaker Bay, BB; Kapiti Island, KP; South Coast, SC; Matiu-Somes Island, SI) of Tethya burtoni in central New Zealand. Plots labelled ' $A$ ' correspond to the analysis run excluding clones, where ' $\mathrm{B}$ ' includes clones for the Breaker Bay population. Top: DAPC based on microsatellite allele frequencies, where each point represents a $T$. burtoni genotype and populations (BB, KP, SC, SI) are labelled within $67 \%$ of their inertia ellipses. Bottom: STRUCTURE plots, where each vertical 
line represents a sponge and the coloration corresponds to the estimated percent chance that an individual belongs to each of the genetic clusters (K). $K=3$ for STRUCTURE plot ' $A$ ' and $K$ $=4$ for plot ' $\mathrm{B}$ '. Clone groups labelled in ' $\mathrm{B}$ ' plots $(1,2,3$; see Appendix 7 for more information on clone groups)

Figure 5.5. Clone groups of Tethya burtoni found at Breaker Bay. Numbers (1-4) and labels (BB\#) correspond to clone group and sponge IDs, respectively (see Appendix 7, Table A7) for more information on clone groups). Sponge IDs written below the sponge that it corresponds to, unless otherwise indicated by an arrow. Newly fallen buds that settled and grew over the monitoring period labelled. White lines in bottom right corner of each picture represent scale bar $=20 \mathrm{~mm}$. Also pictured but not relevant to this study are T. bergquistae, which is an orange or pink golf ball sponge.

Figure 5.6. Images of sections from sexually reproductive specimens Tethya burtoni. Left: Female sponge containing oocytes (o). Right: Male sponge containing spermatic cysts (sc).

Figure 5.7. Size frequency distribution of Tethya burtoni. Size classes broken by every mm. Grey bars correspond to non-reproductive sponges, and black bars represent reproductive sponges. Each symbol above the bars (either asterisk or number) corresponds to a clone sampled from clone groups, with the sampled clone placed above its size class (see Appendix 7, Table A7 for information on clone groups). An asterisk means that the clone was nonreproductive, whereas a ' 1 ' means that the clone contained gametes (either oocytes or spermatic cysts).

Figure 6.1. Examples of images of Tethya burtoni found in the literature and guidebooks. Citations: A = Battershill et al. (2010); B = from NIWA's identification guide by Kelly and Herr (2015); C = Pritchard et al. (1984); D = an image of the original description of T. burtoni in Sarà and Sarà (2004)

Figure 6.2. Examples of images of Tethya burtoni and T. bergquistae observed in this study. Sponge in A \& B are T. burtoni, while sponges in D are T. bergquistae (based on genetic analyses in Chapter 2). Figure $\mathrm{C}$ shows a group of both $T$. bergquistae and $T$. burtoni, where $T$. bergquistae are identified using the number 1 and the rest (unlabelled) are T. burtoni.... 149

Figure 6.3. Genetic division between the Tethya burtoni complex, where population codes are: $\mathrm{BB}=$ Breaker Bay, SC = South Coast, SI = Matiu-Somes Island, KP = Kapiti Island. Those populations with a ' 1 ' belonged to Clade 2 produced by $\mathrm{rnl} / 18 \mathrm{~S}$ and those with a ' 2 ' belonged to Clade 3 produced by $\mathrm{rnl} / 18 \mathrm{~S}$ (see Chapter 2, Figures $2.2 \& 2.3$ ). Each point in the discriminant principal coordinate analysis represents a sponge genotype which are encompassed within $67 \%$ of their population inertia ellipse. 151

Figure 6.4. STRUCTURE plots comparing population structure of only one species of $T$. burtoni (A, plot from Chapter 5, Figure 5.4) to the T. burtoni complex (B \& C, new plots). Each vertical line corresponds to a sponge, and the proportion of colour is the probability of 
assignment into that colour's genetic cluster $(K)$. For plot $A, K=3$. For plot $B, K=2$ and for plot $C, K=6$. For plots $B+C$, the plots contain the same individuals in the same order, and plots are aligned so that the same individual can be read from $\mathrm{B}$ directly down to $\mathrm{C}$. 153

Figure 6.5. Percent survival for Tethya bergquistae and T. burtoni at different temperature treatments (A-B) and associated Kaplan Meier survival curves (C-D). The control temperature was the $17^{\circ} \mathrm{C}$ treatment, indicated by the symbol '(C)'. .160

Figure 6.6. Observations over two days of a sea star (S) preying on Tethya burtoni, where the picture on the right shows remains of digested sponge tissue.

Figure A3. Pairwise correlations between monthly means of sea surface temperature (temp), total monthly rainfall (rain), and monthly means of chlorophyll- $a$ concentration (chla)

Figure A4. The effects of site (top) and species (bottom) on ROI for Tethya bergquistae (purple) and T. burtoni (green) from both Breaker Bay (blue) and Princess Bay (orange). ROI, or the reproductive output index, corresponds to the percent of tissue containing oocytes. Plots on left are means of the $\log (\mathrm{ROI})$ between site (A) and species (C), and bars are $95 \%$ confidence intervals. Plots on right are differences in ROI between sites (B) and species (D) presented by month, where the entire reproductive event captured is highlighted in grey. Sample sizes for species collected from each site are presented in Table 3.1. Error bars on plots on right correspond to standard error.

Figure A6a. Number of buds over time for each monitored Tethya bergquistae (sponge ID indicated by tag number in legend) for tagged sites. Each number (1-6) represents a site, each graph represents a tag, and each line represents a tagged sponge.

Figure A6b. Number of buds over time for each monitored Tethya burtoni (sponge ID indicated by tag number in legend) for tagged sites. Each number (1-6) represents a site, each graph represents a tag, and each line represents a tagged sponge. 



\section{LIST OF TABLES}

Table 2.1. Primer information for markers used (18S, rnl, and COI-ext). Forward and reverse sequences presented $5^{\prime} \rightarrow 3^{\prime}$. Product sizes are in base pairs. Accession numbers refer to distinct haplotypes and genotypes that were deposited to GenBank for each marker.

Table 2.2. Genetic divergence (p-distances, \%) for Tethya spp. for all gene markers. Intraclade p-distances are reported for individuals within clades, while interclade values are pairwise comparisons between all clades.

Table 2.3. Mean lengths for spicules in Tethya bergquistae (Clade 1) and T. burtoni (Clades 2 +3 ) for three genetic clades identified by $18 \mathrm{~S}$ and rnl (Figures 2 and 3) and for all samples combined (overall). Strongyloxea are presented as length $\times$ width. R:C refers to ray length:diameter-to-centre ratios. All sizes are in microns $(\mu \mathrm{m})$.

Table 2.4. Summary of genetic divergence (p-distances, \%) for various sponge species. Ranges given for intraclade and interclade $\mathrm{p}$-distances.

Table 3.1. Summary information of Tethya spp. collected from Feb 2015-2017. Site refers to Breaker Bay (BB) or Princess Bay (PB). Number of sponges collected (N), number containing oocytes (N-O), and number containing spermatic cysts (N-SC) given for each site, each species, and overall. Mean sponge size refers to the diameter of the sponge $(\mathrm{mm})$. Mean oocyte density (counts per $\mathrm{mm}^{3}$ ) and mean oocyte size (SA of oocyte, $\mu \mathrm{m}^{2}$ ) are averaged across all months. The average reproductive output index (ROI) is given as the $\%$ of tissue sampled with reproductive entities. Standard deviations given for all values.

Table 4.1. Summary information of collected Tethya spp. from Feb 2015-2017. Site refers to Breaker Bay (BB) or Princess Bay (PB). Number of sponges collected (N) and number of sponges containing buds (N-B) given for each site, species, and overall. Mean bud density in counts per $\mathrm{cm}^{2} \pm \mathrm{SD}$ averages for all sponges containing buds across all months.

Table 5.1. Microsatellite information with locus name, repeat motif, forward and reverse primer sequences (presented $5^{\prime} \rightarrow 3^{\prime}$ ), number of alleles $\left(\mathrm{N}_{\mathrm{A}}\right)$, size range of products (in base pairs), annealing temperature $\left(\mathrm{T}_{\mathrm{A}}\right.$, in $\left.{ }^{\circ} \mathrm{C}\right)$.

Table 5.2. Basic genetic diversity indices for all loci for each population of Tethya burtoni, with sample size given after each population name. For each locus, the number of alleles $(\mathrm{Na})$, observed heterozygosity (Ho), expected heterozygosity (He), inbreeding coefficient ( $F_{\text {IS }}$ ), and the P-value for deviations from Hardy-Weinberg equilibrium (P-HWE) are given. Values in parentheses are for the Breaker Bay population including clones. Bolded and starred P-values indicate significance. All P-values adjusted using standard Bonferroni correction.

Table 5.3. Basic genetic diversity indices for each population, which includes: the number of samples per population $(\mathrm{N})$, the average number of alleles in each population (mean NA), the average number of alleles after rarefaction to account for small sample size (mean NA after 
rarefaction), the number of private alleles (NPA), inbreeding coefficients $\left(F_{\text {IS }}\right)$, significance for inbreeding (P-F $F_{\mathrm{IS}}$ ), observed heterozygosity (Ho), expected heterozygosity (He), and significance for deviations from Hardy-Weinberg equilibrium (HWE). NS indicates not significant, and $* *$ indicates significance $(P<0.0001)$. Populations are as follows: Breaker Bay excluding clones $(\mathrm{BB}-\mathrm{C})$ and including clones $(\mathrm{BB}+\mathrm{C})$, Kapiti Island $(\mathrm{KP})$, Matiu-Somes Island (SI), and South Coast (SC). Numbers without parentheses are for analyses including all 11 microsatellite loci, and numbers in parentheses are for analyses including only loci in HWE.

Table 5.4. Population pairwise $F_{\mathrm{ST}}$ values, where bolded values in grey indicate significant differentiation $(P<0.0001)$. Populations are as follows: Breaker Bay excluding clones $(\mathrm{BB}-$ $\mathrm{C})$, Breaker Bay including clones $(\mathrm{BB}+\mathrm{C})$, Kapiti Island (KP), Matiu-Somes Island (SI), and South Coast (SC). Two tables represent analysis excluding clones (A) versus analysis including clones at Breaker Bay (B).

Table 5.5. Results from an analysis of molecular variance (AMOVA), where regions refer to the differences between the Kapiti Island population and the Wellington region (WR) populations. Populations in the WR include: Breaker Bay (BB), Matiu-Somes Island (SI), and the South Coast (SC). Two tables represent analysis excluding clones (A) versus analysis including clones at Breaker Bay (B).

Table 6.1. Information on reproduction for sponge species from temperate Australasia. Time refers to reproductive timing, which is either discrete/seasonal (S) or continuous (C). Gamete production (prod) refers to either gonochorism $(\mathrm{G})$ or hermaphroditism $(\mathrm{H})$, and gamete development (dev) refers to either ovipary (O) or vivipary (V). Reproductive output index (ROI) refers to the proportion of sponge occupied by gametes (given as \%). Densities given in counts (oocytes, embryos, larvae or spermatic cysts) per $\mathrm{mm}^{2}$, and all data were adjusted to be presented in $\mathrm{mm}^{2}$. Ranges given for each numerical column, except when only one number presented, which is the maximum value observed for that sponge. NF refers to gametes not being found, and - refers to information not available. For location, NZ = New Zealand and $\mathrm{W}-\mathrm{AU}=$ Western Australia.

Table A1. Information on collected specimen for Chapter 2. Colour refers to colour of sponge, where $\mathrm{P}=$ pink and $\mathrm{Y}=$ yellow. All sampling sites (Breaker Bay, BB: 41 ${ }^{\circ} 19^{\prime} 53.3^{\prime \prime} \mathrm{S}$ 174²9'52.6"E; Kapiti Island, KP: 4053'23.6"S 174²'40.3"E; Matiu-Somes Island, SI: $41^{\circ} 15^{\prime} 36.9^{\prime \prime S} 174^{\circ} 43^{\prime} 54.4^{\prime \prime E}$; Red Rocks, RR: 41 $\left.21^{\prime} 04.5^{\prime \prime S} 174^{\circ} 43^{\prime} 54.4^{\prime \prime E}\right)$ are in New Zealand. All specimens were collected from 5-10 m depth. Phylogenetic clade refers to clades on individual gene phylogenetic tree. GenBank Accession numbers are given in parentheses after haplotype ID.

Table A5a. Results of likelihood ratio chi-square tests, testing the effects of site, sponge size, species, sea surface temperature, rainfall and chlorophyll- $a$ concentration on the probability of a sponge containing gametes (Chapter 3 ). On the left, temperature as a continuous variable; on the right, temperature as a categorical variable. 
Table A5b. Results of an ANOVA testing effects of site, sponge size and species on oocyte density, ROI (or a reproductive output index, or the $\%$ of tissue occupied by gametes) and oocyte size (Chapter 3).

Table A5c. Results of likelihood ratio chi-square tests, testing the main effects of site, sponge size, species, sea surface temperature, rainfall and chlorophyll- $a$ concentrations on the probability of a sponge containing buds (Chapter 4). On the left, temperature as a continuous variable; on the right, temperature as a categorical variable.

Table A5d. Results of likelihood ratio chi-square for effects from the best fit model for the probability of budding. Species refers to either Tethya bergquistae or T. burtoni, site refers to either Breaker Bay or Princess Bay (Chapter 4). Sea surface temperature (SST) in ${ }^{\circ} \mathrm{C}$ and chlorophyll- $a$ concentrations in $\mathrm{mg} / \mathrm{m}^{3}$.

Table A5e. Results from a Type-III ANOVA, testing the main effects of site, sponge size, species, sea surface temperature, rainfall and chlorophyll- $a$ concentrations on bud density (numbers per $\mathrm{cm}^{2}$; Chapter 4).

Table A5f. Results from a Type-III ANOVA for effects from the best fit model for bud density. Species refers to either Tethya bergquistae or T. burtoni, site refers to either Breaker Bay or Princess Bay. Sea surface temperature (SST) in ${ }^{\circ} \mathrm{C}$ and chlorophyll- $a$ concentrations in $\mathrm{mg} / \mathrm{m}^{3}$ (Chapter 4).

Table A7. Clone groups of Tethya burtoni found at Breaker Bay, with information for clone group ID (1-4); sample ID (i.e. BB\#); gametes observed ( 0 = no gametes, 1 = gametes present, $\mathrm{O}=$ oocytes, $\mathrm{SC}=$ spermatic cysts); ROI (or reproductive output index, which is a measure of reproductive effort and is calculated as the percent of tissue occupied by gametes over the total tissue area); budding observed ( $0=$ no budding, $1=$ buds present); and budding period (time of budding events). Duration of the monitoring period, as well as times monitored (X mon), for each clone group are also given. Square area containing all clones is given in $\mathrm{cm}^{2}$, along with radial area (radial area around the sponge containing gametes to the point where all other clones are encompassed). Sponges in grey were mortalities over the monitoring period.....225

Table A8. Linkage disequilibrium observed across populations of Tethya burtoni for analysis excluding clones (without clones) and including clones (with clones), for all microsatellite loci and for only those loci in HWE. 

CHAPTER 1:

GENERAL INTRODUCTION 



\section{The conundrum of sex}

Sex is one of the greatest conundrums for evolutionary ecologists. The persistent selection of sexual reproduction remains puzzling because it often fails to follow the rule of natural selection, which defined by Darwin in 1859 is "the principle by which each slight variation, if useful, is preserved" (from On the Origin of Species). Organisms have two main ways to reproduce, sexual and asexual reproduction. Sexual reproduction involves recombination and results in genetically distinct offspring, whereas asexual reproduction results in identical clones. Of the two modes of reproduction, sexual reproduction is costlier, yet despite this, still remains frequently and constantly employed by most organisms (Doncaster et al., 2000; Lehtonen et al., 2012; Roze, 2012). There is an intrinsic two-fold cost of sex, described first by Maynard Smith $(1971,1978)$, which encompasses both the cost of males (i.e., where males cannot produce offspring), and the cost of meiosis (i.e., where only half of the parental genetic material is passed to the next generation). This gives asexual organisms a two-fold advantage over sexual ones because by contrast, all asexual organisms have the ability to produce offspring and pass on all of their genes to the next generation. Sex can also be unfavourable because recombination, which occurs when chromosomes cross over during meiosis, has the potential to break up beneficial genetic combinations that would otherwise allow offspring a better chance of survival (Uecker and Hermisson, 2015). However, during asexual reproduction, all alleles are linked and inherited together, which would allow 'fit' genotypes to persist. Sexual reproduction also results in costs associated with the mating process, as these increase the chances of predation, parasite transmission and injury, which would also reduce future reproductive success (Daly, 1978; Charlesworth, 1980). Further, gametes are costly because additional resources are needed for various stages of gametic production and development (Hayward and Gillooly, 2011). Asexual reproduction does not have these disadvantages associated with the mating process, which would further make the selection of this mode of reproduction more logical from an evolutionary perspective. Despite these seemingly unfavourable characteristics of sexual reproduction, it nonetheless has been persistently selected for across many different organisms and is more frequently observed in nature (Andersson, 1994).

While there are potential negative consequences of recombination (i.e., the dissociation of beneficial gene combinations), sexual reproduction is thought to be selected for in nature for 
the sole reason of recombination, which also acts to generate greater genetic variation than point mutations alone (Uecker and Hermisson, 2015). When pieces of chromosomes are exchanged during recombination, new alleles are formed. This genetic diversity provides a higher adaptability potential for 'fitter' genotypes in an array of environments (Lande and Shannon, 1996; Barton and Charlesworth, 1998). Beneficial mutations can occur during asexual reproduction (i.e., through base pair changes during DNA replication), but the rate at which this occurs is very slow and further, asexual mutants must compete with other asexuals whose genotypes are already adapted to the environment (Gerrish and Lenski, 1998). In addition, while recombination can break up beneficial gene combinations, it can also act to break up disadvantageous ones. Asexual reproduction has no mechanism for regulating deleterious mutations, and as such, mutations accumulate exponentially and in an irreversible manner in clonal populations. This idea, coined the 'ratchet effect' by Muller (1932), has been deemed as one of the most important reasons why sex persists in populations. Furthermore, the lack of genetic diversity associated with asexual reproduction can lead to inbreeding depression (Muirhead and Lande, 1997), which would make populations more prone to bottlenecks or population collapses. While it is generally thought that asexual reproduction results in lower genetic diversity (e.g., Ayre, 1984; Burdon and Roelfs, 1985), there are organisms that asexually reproduce that challenge this hypothesis. For instance, there are studies showing that asexual populations can show considerable diversity and sometimes are actually more diverse than strictly sexual populations (e.g., Infante et al., 2003; Grapputo et al., 2005; Shreve et al., 2011). Many models have been proposed to explain this apparent paradox, focusing on how each mode contributes to genetic diversity for populations, and how this in turn may influence population dynamics. For instance, Bengtsson (2003) examined populations that reproduce both sexually and asexually, and hypothesized that variation in clonal populations is generated by the number of sexual events. Under neutral selection, this model predicts the number of sexual events needed in clonal populations to generate the same allelic and genotypic diversity as strictly sexually reproduction populations. Similarly, Barbuti et al. (2012) proposed that sexual reproduction serves to generate 'fit' genotypes, whereas asexual reproduction serves to amplify such genotypes. Understanding how both modes shape the genetic diversity of a population in nature, however, remains challenging.

The environment is often the driving force that influences which reproductive mode is employed by an organism, as ultimately dictated by natural selection. Asexual reproduction is 
commonly associated with more stable environments because homogeneous environments do not require an array of adaptable genotypes to various conditions (Bürger, 1999). In contrast, sexual reproduction is thought to be important for populations living in constantly changing environments, where genetic variation (via recombination) is important for allowing the population to adapt and persist, especially to new environments (Crow, 1992; Becks and Agrawal, 2012). Thus, asexual reproduction and sexual reproduction are generally associated with stable and heterogeneous environments, respectively (e.g., observed in populations of sea anemones, Actinia tenebrosa (Ayre, 1984); and populations of marine sponges, Chondrilla sp. (Zilberberg et al., 2006)). Despite this, instances of asexual reproduction occurring in heterogeneous environments and instances of sexual reproduction occurring in stable environments have been reported. In highly disturbed areas, high incidences of asexual reproduction have been reported (e.g., Sherman et al., 2006), where the exponential amplification of clones proves beneficial for recolonization following disturbance (Rasheed, 2004; Silvertown, 2008). By contrast, in extremely stable environments, repetitive and frequent asexual reproduction may more quickly lead to Muller's ratchet and therefore sex is eventually required to prevent population extinction by the ratchet effect (Doncaster et al., 2000). Overall, there are no general rules for either sexual or asexual reproduction in terms of which mode is more advantageous, how each mode contributes to genetic diversity, or in what environment each mode is likely to be employed. It is instead specific to a particular organism living in a certain environment at a particular time, and it is this transient nature of the science that drives the great debate surrounding sex in evolutionary ecology.

\section{Connectivity and species boundaries in the marine realm}

Connectivity, broadly defined, is the exchange of individuals between populations (Cowen and Sponaugle, 2009). More specifically, connectivity can be thought of in terms of both demographic connectivity and genetic connectivity, as reviewed in Lowe and Alldendorf (2010). As defined by these authors, demographic connectivity assesses population growth in terms of the relative contribution of both net immigration and local recruitment. By contrast, genetic connectivity measures the total number of organisms that disperse between populations, but fails to capture the rate at which this occurs and thus cannot give an indication of population growth without necessary demographic data. For organisms living in the marine environment, connectivity patterns are a product of both biotic and abiotic forces (i.e., 
reproductive strategies, larval duration/dispersal; Cowen et al., 2007; Pineda et al., 2007). These processes are often unknown or are difficult to study in the ocean, making predicting connectivity challenging. For instance, it was previously thought that marine populations were connected across larges distances (i.e., open populations), but more recent data are beginning to reveal that populations are often more closed off, with restricted gene flow and greater structure between populations (Cowen et al., 2000; Warner and Cowen, 2002). A key factor that shapes a species' or a population's connectivity patterns and associated genetic structure is their reproductive ecology (Cowen and Sponaugle, 2009; Treml et al., 2012). For example, organisms that use mixed reproductive strategies may rely on asexual reproduction as a repeated seeding strategy, resulting in clonal patches of individuals that are restricted to a small spatial scale (Ellstrand and Roose, 1987; Alberto et al., 2005). Additionally, sexual reproduction combined with a low dispersal of offspring may result in local recruitment, causing inbred populations (Keller and Waller, 2002). For those organisms that have complex reproductive strategies with both sexual and asexual reproduction, the contribution of each mode to connectivity is often unknown, which impairs our understanding of how such populations are structured. In addition to reproductive behaviour, connectivity is also greatly influenced by the surrounding environment, where oceanographic currents, tides, and topological barriers operate to either promote or restrict connectivity (Galarza et al., 2009; Paris et al., 2007). Determining both temporal and spatial connectivity patterns is fundamental for not only our understanding of the population dynamics and evolutionary ecology of an organism, but it is also valuable for effective management and conservation of marine systems.

When predicting connectivity patterns for an organism, the identification of the species of interest is generally the first step in a study, and this step is assumed to have been done correctly. However, while seemingly basic, identifying species boundaries in ecology is not trivial and remains one of the greatest challenges for marine species (Wheeler and Meier, 2000). For the marine environment in particular, barriers to reproduction are not obvious, and intraspecific plasticity in combination with interspecific cryptic diversity makes delineating species difficult. As such, the use of integrative taxonomy which uses more than only morphological analyses is recommended in order to better understand the evolutionary history of an organism (Padial et al., 2010). Recent molecular studies have begun to uncover many cryptic species in the ocean (Knowlton, 2000). Cryptic species are defined as two or more species that have been classified as one species on the basis of morphology, despite being in 
fact evolutionarily distinct species (Bickford et al., 2007). While the identification of cryptic species is most usually by genetic tools (Knowlton, 2000), species may also be deemed cryptic on the basis of reproductive isolation (Amato et al., 2007), as well as differences in chemical defence systems and endosymbionts (Mcgovern and Hellberg, 2003). However, such differences are sometimes subtle and as a result, the definition of species can often be subjective, which has led to intense debates on species delineations in taxonomic fields (Hauser, 1987; Wheeler and Meier, 2000; Will and Rubinoff, 2004). It is difficult to determine at what point strongly differentiated populations are considered different species, which can confuse our understanding of populations, subpopulations, sibling species and even species. Failure to correctly identify the former has consequences in connectivity studies, as misinterpretation of species boundaries can lead to erroneous estimates of gene flow (Pante et $a l ., 2015)$. Sympatric cryptic species perhaps have the most elusive evolutionary histories, as it is often difficult to identify barriers to reproduction between species occupying the same niche with the seemingly same functional role (Knowlton, 1993; Rundell and Price, 2009). In addition, for species living in sympatry, boundaries can be semipermeable, where processes including introgression and hybridization confound and blur species delineations (Harrison and Larson, 2014). Introgression, or the process by which genes from one species are transferred to another species through hybridization and backcrossing, has recently been shown to occur more frequently in marine systems than previously thought (Roques et al., 2001; Soliva and Widmer, 2003; Nydam and Harrison, 2011). The basis of any evolutionary study is the identification of the correct study species, and because this is a seemingly simple task, it is often overlooked in many studies.

\section{Sponges as model organisms}

Sponges, which belong to the phylum Porifera, can serve as useful models for evolutionary studies. They are among the most ancient multicellular animals, with an evolutionary history dating to the Pre-Cambrian period, approximately 600 mya (Li et al., 1998; Yin et al., 2015), and they continue to thrive today. They are an incredibly diverse group with over 9,000 species identified to date, with new species continually being discovered (Van Soest et al., 2012; Van Soest et al., 2018). This group exhibits an array of both morphological types (with regard to skeletal composition, growth forms) and life history strategies, with wide variations in growth, reproduction, and feeding (Frost, 1976; Bergquist, 1978; Hooper and Van Soest, 2002; 
Maldonado and Riesgo, 2008). Despite the high levels of diversity within the group, all sponges serve largely to filter water, and their simple body plan reflects this (Bergquist, 1978). In general, filtration occurs as water is drawn into the sponge, circulated and expelled (Leys et al., 2011). Despite the simplicity, sponges have persisted for millions of years and serve a number of ecologically important roles in the benthos of both freshwater and marine environments, across tropical, temperate and polar oceans (Van Soest et al., 2012). Sponges have been shown to play important functional roles in reef/habitat creation, bioerosion, trophic webs and nutrient cycles; furthermore, sponges support primary production, produce chemical defences and provide shelter for a range of organisms (Bell, 2008). For studies requiring fieldwork, sponges are ideal study species as they are sessile and have fast regeneration rates (Wulff, 2010; Figure 1.1). Of the most interesting characteristics for evolutionary studies, however, is the diverse way in which sponges reproduce.
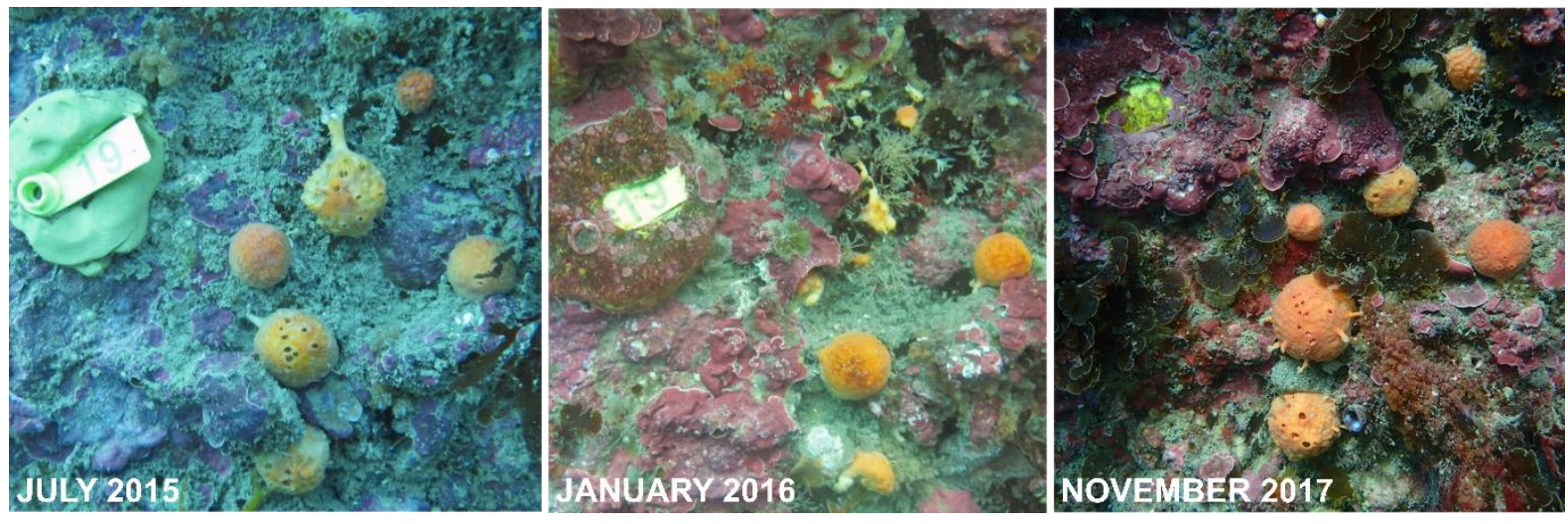

Figure 1.1 Regeneration of sponges used in this thesis over a two-year study period.

\section{Sponge reproduction}

Sponges reproduce using both sexual and asexual reproduction (as recently reviewed in Maldonado and Riesgo, 2008). During sexual reproduction, sponges can be gonochoristic (i.e., separate sexes) or hermaphroditic (i.e., produce both male and female gametes). Hermaphroditism can be simultaneous, where both gametes are present at the same time, or sequential, where one sex develops into the other (Sara, 1974; Gilbert and Simpson, 1976). In terms of gamete development, sponges either release eggs into the water column where they are fertilized and develop into larvae (i.e., ovipary, as in Polymastia sp.; Ayling, 1980) or internally brooded larvae (i.e., vivipary, as in Haliclona sp.; Fromont, 1994). Sponges have three main ways of asexual reproduction: budding, fragmentation or gemmulation (Simpson 
and Gilbert, 1973; Maldonado and Uriz, 1999; Cardone et al., 2010). Budding occurs when sponges release small cell masses, or buds, from their external surface. Buds are composed of fibrils, totipotent cells (called archeocytes), energy stores and spicules to allow longevity in the water column as well as settlement to substrate (Simpson, 2012). Fragmentation occurs when a small piece of tissue is detached from the sponge (via water movement, or interactions from other organisms) and resettles, growing into a new sponge (Maldonado and Uriz, 1999; Wulff, 1991). Because many cells that make up a sponge are pluripotent, or can develop into all cell types, only small pieces of sponge fragment are necessary to allow regeneration of an entire sponge (Simpson, 2012). The third mode of asexual reproduction, gemmulation, occurs when sponges release gemmules, which are small dormant cell masses located at the based of the sponge surrounded by a protected envelope (Rasmont, 1962). Gemmules are usually associated with extreme tissue damage and disturbance events, as the thick protective envelope allows resistance to desiccation events and even digestion by other animals (Simpson and Gilbert, 1973). Gemmulation is generally more frequent in freshwater and brackish habitats, and is a mechanism by which populations can be restored after mass mortalities from freezing and drought events (Simpson and Fell, 1974). Sponges have evolved to use combinations of these sexual and asexual strategies. For example, some sponges are oviparous gonochorites that bud (e.g., Tethya, Bergquist and Kelly-Borges, 1991), while others are viviparous hermaphrodites that undergo fragmentation (e.g., Haliclona, Wulff, 1986; Stephens et al., 2013). The high diversity of sexual and asexual reproduction provides an excellent opportunity to identify potential roles of different reproductive strategies in population dynamics and in the overall evolutionary history of an organism.

Both sexual and asexual reproduction in sponges are greatly influenced by the environment. Multiple studies have shown that various environmental factors (including temperature, nutrient/food availability, salinity, rainfall, oxygen, sediment/turbidity, light) correlate to the reproductive timing, frequency and overall output in sponges. Of potential environmental drivers, temperature is the most studied, and both increases and decreases in temperature have been shown to coincide with the onset of reproduction. For example, gamete production and budding has been shown to occur during periods of warmer temperatures for a number of sponges, including: Carteriospongia foliascens (Abdul Wahab et al., 2014), Xestospongia spp. (Fromont and Bergquist, 1994), Geoida cydonium (Mercurio et al., 2007), Halichondria panacea (Witte et al., 1994) and Tethya citrina (Cardone et al., 2010). Conversely, decreasing 
temperatures are correlated with reproductive and budding events for other sponges, as observed in Axinella damicornis (Riesgo and Maldonado, 2008) and Mycale contrarenii (Corriero et al., 1998). Light and lunar/tidal cycles are also important and often trigger spawning events for sponges (Amano, 1988; Nozawa et al., 2016). Nutrient levels are often used as a proxy for food, which is important for gamete production, and thus nutrients and food availability have been found to be positively correlated with reproductive output (i.e., gamete density). By contrast, gamete production is negatively affected by decreases in salinity and oxygen (Witte, 1996; Roberts et al., 2006; Gaino et al., 2010; Di Camillo et al., 2012). Furthermore, stress from sedimentation may result in the allocation of energy to other survival strategies, leaving less energy available for reproduction, which generally negatively affects reproduction in sponges (Whalan et al., 2007b). For example, Roberts et al. (2006) reported a decrease in the number of gametes produced by Cymbastela concentrica under increased sediment and low light conditions. Understanding how sponges respond to the surrounding environment is imperative for understanding population dynamics, including connectivity, as these processes are driven by reproduction. Furthermore, with predicted shifts in our climate, understanding environmental drivers of sponge reproduction is imperative in order to predict how these populations will respond to changing oceans and how to effectively manage them.

\section{Sponge taxonomy}

Knowledge of an organism's reproductive ecology can be informative in setting species boundaries, as species are often defined as being reproductively isolated from one another. Traditionally, taxonomy of sponges has been on the basis of comparative morphology, focusing on differences in the skeletal and spicule composition between species (Hooper and Van Soest, 2002). However, due to the simplicity of sponges, there may sometimes be insufficient morphological characters available to distinguish species from each other. Furthermore, conspecific sponges often exhibit high degrees of phenotypic plasticity and can appear very different, when they are in fact the same species (Gaino et al., 1995). Due to these challenges, the field of sponge taxonomy is known for being particularly difficult, even for experts (KellyBorges and Pomponi, 1992; Hooper and Van Soest, 2002; Wörheide and Erpenbeck, 2007). Increasingly, molecular analyses have begun to uncover cryptic species throughout the phylum, as the high diversity of sponges that lack defining traits coupled with their phenotypic plasticity, make the potential for cryptic species very likely. For example, De Paula et al. (2012) 
discovered four species within what was previously Cliona celata in South America using genetic markers, even though spicule analysis failed to differentiate species in this complex. Similarly, discordance between molecular and morphological species boundaries was recorded in Callyspongia in the Caribbean (DeBiasse and Hellberg, 2015). Genetic evidence has also revealed cryptic species within calcareous sponge species (Solé-Cava et al., 1991; Wörheide et al., 2008), Placospongia (Nichols and Barnes, 2005), Haliclona caerula (Knapp et al., 2015), and Hemimycale (Uriz et al., 2017). Hybridization, introgression and chimerism between species have also been recorded in the phylum Porifera (e.g., Maldonado, 1998; Riesgo et al., 2016), making the interpretation of genetic data difficult. While genetic data are beginning to reveal evolutionary relationships between sponges, it is still important to take a holistic approach to sponge taxonomy as genetic variability between groups may merely be a product of subpopulations or intraspecific diversity. As such, taxonomically diagnostic characters, such as those relating to morphology and reproductive ecology, in combination with genetic analyses may provide a more rounded view of species' evolutionary histories. For example, Muricy et al. (1996) found cryptic speciation within Plakina trilopha on the basis of genetic evidence, reproductive isolation and morphology. Delineations of species remains a difficult task, especially for sponges, and the dynamic field of sponge taxonomy reflects this, where new studies are constantly highlighting hidden diversity within the phylum.

\section{Sponge connectivity}

There are a number of studies that have examined connectivity patterns of sponges. Larval duration and behaviour can provide clues as to how populations are connected; for instance, long-lived, swimming larvae are better able to connect populations at greater distances than immobile, short lived larvae (Maldonado, 2006). This has been shown to be the case for Scopalina lophyropoda, where philopatric larval dispersal resulted in genetically differentiated populations across both small and large scales (Blanquer and Uriz, 2010). Another technique that has been used to examine the extent of gene flow is graft acceptance or rejection (Neigel and Avise, 1983). For instance, this technique has been used by Jokiel et al. (1982) for Callyspongia diffusa, and involved taking grafts from one population, putting them into contact from individuals of another population, and calculating the frequency of acceptance and rejection. These authors found that graft acceptance was negatively correlated with distance from the source of the graft, allowing the extent of gene flow and the level of clonality to be interpreted. Most connectivity patterns in sponges, however, have been determined using 
genetic markers, which includes the use of allozymes, mitochondrial/nuclear universal markers, multilocus microsatellite markers, and single nucleotide polymorphisms (SNPs; Van Oppen et al., 2000). Allozymes and mitochondrial/nuclear markers have been used extensively in multiple studies looking at population structure (Klautau et al., 1999; Duran et al., 2004a; DeBiasse et al., 2010; Lopez-Legentil and Pawlik, 2009), but can sometimes fail to capture genetic differentiation between sponges from different locations, due to the conservative nature of these markers (Bucklin et al., 2011). Microsatellite markers and SNPs are both advantageous in that they capture fine scale intraspecific relationships of sponges, allowing for a better indication of population structure. Duran et al. (2004b) was the first study to develop microsatellite markers for a sponge (Crambe crambe), and since a number of studies have emerged using this genetic tool to determine connectivity patterns. These studies have revealed a common pattern for sponges, where populations are highly differentiated across short distances and have more restricted gene flow than originally expected (Perez-Portela et al., 2014; Blanquer and Uriz, 2010; Duran et al., 2004b). Such restricted gene flow and local recruitment has been attributed to oceanographic barriers and fronts (e.g., Riesgo et al., 2016) as well as short larval duration times (e.g., DeBiasse et al., 2010). Some populations are connected across larger distances than expected, as in the case of some populations of Dendrilla antarctica in Antarctica (Carlos et al., 2017). For such open populations, oceanographic currents can drive populations to be connected across larger distances (e.g., Lazoski et al., 2001). While information surrounding gene flow in sponges is growing, connectivity patterns can be hard to disentangle in this phylum, especially because cryptic speciation has the potential to confound such gene flow scenarios and lead to misinterpretation of data.

Connectivity patterns can change, demonstrating that environmental influences are important in determining connectivity patterns. Examining genetic structure and differentiation over a short time scale can reveal such temporal components to connectivity patterns. For example, the population structure (i.e., genetic structure and clonality) of the sponge Paraleucilla magna was determined for three consecutive years and showed great change over the three years (Guardiola et al., 2011). For the first and third sampling years, there were three genetically distinct populations; however, for the second year, genetic differentiation decreased and only two distinct populations were detected. This study highlighted that connectivity patterns can be affected by both time and the surrounding environment. Therefore, determining how reproductive modes are influenced by the environment is key to predicting such changes in 
connectivity. For instance, Zilberberg et al. (2006) correlated the extent of clonality to surrounding environmental conditions, where clonality was higher in more stable environments. As such, asexual reproduction was attributed to shaping the genetic structure of this population, whereas sexual reproduction was greater in heterogeneous environments. The reliance on asexual reproduction by sponge population varies. Asexual reproduction can play a minor role in sponge populations, such as those of Scopalina lophyropoda in the Mediterranean Sea (Blanquer and Uriz, 2010); or can instead play a key role in population persistence, as for some Caribbean sponges (Wulff, 1991). Understanding how the environment influences both sexual and asexual reproduction is imperative to predicting connectivity patterns, because gene flow is driven by reproduction. While reproduction and potential environmental drivers have been recorded for many sponges, how this interaction drives connectivity patterns and shapes the evolutionary history of these species remains poorly understood.

\section{Thesis outline}

The overall aim of this $\mathrm{PhD}$ thesis is to better understand how the environment influences complex reproductive strategies in the marine environment, and to determine how different modes of reproduction contribute to evolutionary processes like speciation and connectivity. For this study, I used temperate sponges belonging to the genus Tethya in New Zealand as a model, with the following objectives:

(1) To determine species boundaries between Tethya spp. in central New Zealand. To fulfil this aim, I constructed phylogenetic relationships between Tethya spp. from one nuclear and two mitochondrial markers. I also assessed the use of morphological features as a method to identify species by comparing spicule sizes and types between different species.

(2) To characterize sexual reproduction in Tethya spp. in central New Zealand; to determine any differences between species; and to identify potential environmental drivers of sexual reproduction. To achieve this, I performed histological analysis on sponges collected monthly over two years at two different sites to characterize gametic production and development, as well as sexual reproductive frequency, duration and output. I then compared this data to an environmental dataset, comprised of sea surface 
temperature (SST), rainfall and chlorophyll- $a$ concentration (chl- $a$ ) to determine potential correlates.

(3) To characterize asexual reproduction in Tethya spp. in central New Zealand; to determine any differences between species; and to identify potential environmental drivers of asexual reproduction. I did this by using three different methods. I first measured budding events and bud density for sponges collected monthly over two years at two sites, to which I compared to an environmental data set (SST, rainfall, chl-a). I then also monitored a population of sponges in situ and measured both budding events and bud numbers for the same sponges over two years. I compared these data to temperature data from a logger I deployed in the field. Lastly, I conducted a preliminary experiment looking at budding events in relation to temperature.

(4) To examine the roles of sexual and asexual reproduction in population connectivity for Tethya burtoni across four sites in central New Zealand. To achieve this aim, I developed a set of microsatellite markers to characterize connectivity patterns in this sponge. I also monitored a population of $T$. burtoni monthly, over two years, to investigate the role of asexual reproduction in population dynamics. I did this by measuring the dispersal of asexual buds from genotyped sponges and by using histological methods to determine the sexual ability of clonal individuals.

In the final chapter (General Discussion), I discuss the results from all data chapters together and provide commentary on broader scale implications of this research. I compare the multiple methods employed for species delineation throughout this thesis, including: universal markers and comparative morphology (Chapter 2); sexual reproductive isolation (Chapter 3); asexual reproductive isolation (Chapter 4); and microsatellite markers (Chapter 5). Further, I discuss conditions that may favour evolutionary processes including introgressive hybridization and cryptic speciation. This thesis highlights both speciesspecific and site-speicific responses of sexual and asexual reproduction, and the implications of these results is discussed in relation to climate change. Lastly, this work provides baseline data and tools to be used for further studies, and many future directions exist that can expand on this $\mathrm{PhD}$ thesis. 
CHAPTER 2:

HIDDEN DIVERSITY IN THE GENUS TETHYA: COMPARING MOLECULAR AND MORPHOLOGICAL TECHNIQUES FOR SPECIES IDENTIFICATION

Shaffer MR, Davy SK, Bell JJ (2019) Hidden diversity in the genus Tethya: comparing molecular and morphological techniques for species identification. Heredity, 122, 354-369. 



\section{Introduction}

Correctly identifying species units is critical for estimating biodiversity, defining species boundaries and measuring connectivity patterns (Hey et al., 2003; Frankham et al., 2012). Methods for species identification have been so hotly debated in the literature that the phrase 'species problem' has emerged to describe the conundrum that biologists face when defining a species (Mayden, 1997; De Queiroz, 2007). Mayr (1942) was the first to address this problem by conceiving the biological species concept, where he defined a species as a group of interbreeding individuals which are reproductively isolated from other groups. Since then, other concepts have emerged based on: an organism's functional role/ecological niche (ecological species concept, Van Valen, 1976); historical ancestry lineages (evolutionary species concept, Simpson, 1951); and the sharing of common ancestors/alleles (phylogenetic species concept, Hennig, 1966; Rosen, 1979; Baum and Shaw, 1995; reviewed in De Queiroz, 2007). While many concepts exist, the most traditional, widespread and consistently employed method for taxonomy is based on comparative morphology (Padial et al., 2010).

The recent advancement and accessibility of molecular tools has begun to reveal conflicting evolutionary histories for many species previously defined by morphological characteristics (Wendel and Doyle, 1998; Knowlton, 2000; Lecompte et al., 2005; Lihová et al., 2007; Wallis et al., 2017). Distinguishing between intraspecific plasticity and interspecific cryptic diversity can be problematic when only using morphology-based taxonomic methods (Knowlton, 1993; Vrijenhoek, 2009; Capa et al., 2013, Czarna et al., 2016). Additionally, congeneric species that live in sympatry often challenge the notion of species boundaries because introgression (i.e., where alleles from one species enter the gene pool of another species via hybridization and backcrossing) is common (Harrison and Larson, 2014). Failure to correctly identify species boundaries has consequences for implementing effective conservation and management strategies and for our overall understanding of important evolutionary processes like speciation (Gómez et al., 2002), bioinvasions (Knapp et al., 2015) and hybridization (Kauserud et al., 2006).

Organisms that have only a few defining traits available for comparison are particularly prone to identification problems (Knowlton, 1993; Bickford et al., 2007; DeBiasse and Hellberg, 2015). In such groups, cryptic species often emerge during molecular studies, as sequences 
provide more characteristics (bases) with which to differentiate organisms, which allows a finer resolution of relatedness between individuals to be determined (Hillis, 1987; Hebet et al., 2004; Bickford et al., 2007). Sponges have relatively few morphological traits, yet a rich taxonomic literature exists delineating species boundaries within sponges based on phenotypic characteristics (Hooper and Van Soest, 2002). Skeletal composition, arrangement and spicule morphology are key features used to classify and identify sponges (Hooper and Van Soest, 2002), despite the fact that structural components of sponges can be highly dynamic (Bond, 1992; Nakayama et al., 2015) and spicules can exhibit variability under different environmental conditions (Bavestrello et al., 1993; Bell et al., 2002; McDonald et al., 2002). Furthermore, recent studies on sponge taxonomy have highlighted problems with using morphology as a sole defining trait and have used phenotypic traits combined with molecular analyses to resolve evolutionary relationships (Alvarez et al., 2000; Erwin and Thacker, 2007; Morrow and Cárdenas, 2015; Plotkin et al., 2017). As a result, phylogenetic analyses for many sponges have begun to reveal cryptic speciation throughout the phylum, suggesting that diversity within the phylum is even higher than previously thought (see Table 1 in Xavier et al. (2010) for reported cases of cryptic speciation in sponges, plus Andreakis et al., 2012; De Paula et al., 2012; DeBiasse and Hellberg, 2015; Knapp et al., 2015; Uriz et al., 2017).

In this study, I used 'golf ball sponges' belonging to the genus Tethya in New Zealand as a model to investigate cryptic speciation and to better understand how evolutionary processes occur for species living in sympatry. Tethya is a ubiquitous genus, with 92 described species worldwide (Van Soest, 2008), and has a particularly high diversity (31 species) recorded in coastal waters of Australia and New Zealand (Bergquist and Kelly-Borges, 1991; Sarà, 1998; Sarà and Sarà, 2004; Heim et al., 2007). While members of the genus are easily distinguishable by their globular form, identifying Tethya to the species level can be difficult because they exhibit a high degree of morphological plasticity, and have only a few defining traits available for species differentiation. The challenge in species identification is reflected in the dynamic taxonomic literature, where new species are constantly being described (Corriero et al., 2015), and previously described species are regularly reconsidered and reclassified (Sarà, 1987; Bergquist and Kelly-Borges, 1991; Hooper and Wiedenmayer, 1994; Sarà and Sarà, 2004; Heim et al., 2007). In New Zealand, taxonomy has been based solely on morphology with no genetic phylogenies constructed to date (Bergquist and Kelly-Borges, 1991; Sarà and Sarà, 2004). Here, I used two common species of Tethya in New Zealand (T. bergquistae and T. 
burtoni) as models to investigate evolutionary relationships and cryptic speciation using both genetic (COI-ext, rnl, 18S) and morphological traits (spicules) to understand the advantages and limitations of both methods in determining species boundaries.

\section{Materials and Methods}

Study species

Tethya bergquistae and T. burtoni are two of the most common species of Tethya found in the waters of central New Zealand, and both occur in the waters around Wellington, New Zealand (Figure 2.1). Tethya bergquistae is described as rose-pink in colour, firm to the touch, and with an irregular surface with grouped oscula (Bergquist and Kelly-Borges, 1991; Hooper and Wiedenmayer, 1994; Battershill et al., 2010). Tethya burtoni is bright orange to yellow and is described as having a warty surface with an inflated, soft texture (Sarà and Sarà, 2004; Battershill et al., 2010). Tethya bergquistae is generally found in well-lit areas with moderate flow, whereas T. burtoni tends to prefer more shaded, sedimented areas (Battershill et al., 2010). Spicules for both include megascleres, which are strongyloxeas; and microscleres, which are megasters and micrasters of similar sizes (Bergquist and Kelly-Borges, 1991; Sarà and Sarà, 2004). A key distinguishing feature between both species relies on their spicular composition with respect to the location/density of megasters, as T. burtoni contains fewer megasters, which are mainly found in the cortex (Bergquist and Kelly-Borges, 1991; Sarà and Sarà, 2004).

Tracking the description of both species through the literature reveals a complex taxonomic classification history within New Zealand, highlighting the difficulty in species identification common to Porifera. For instance, $T$. bergquistae was incorrectly identified as $T$. ingalli by Pritchard et al. (1984) and formerly described as T. australis by Bergquist and Kelly-Borges (1991), and T. burtoni has been incorrectly referred to as T. aurantium by Pritchard et al. (1984). Overall in New Zealand, there are ten species of Tethya recorded, but two of these are also thought to exist in Australian waters (Ledenfield, 1888; Bergquist, 1961; Bergquist and Kelly-Borges, 1991; Sarà and Sarà, 2004). Many of these ten species are thought to be endemic to certain regions (e.g., T. compacta to the Chatham Islands, T. fastigata to the Poor Knight Islands), but this has been based on observations from only one to a few sponges (e.g., $T$. 
compacta), or on descriptions dating from the $19^{\text {th }}$ Century (e.g., T. multistella). In the literature, pictures identifying New Zealand Tethya are often inconsistent and classification by comparative morphology is therefore challenging. Descriptions of species within Tethya are based on cross sections and spicule compliments, but differences can be often subtle and difficult to detect, especially for non-specialists. Around New Zealand, sympatric Tethya spp. display a wide range of colours and textures, making them difficult to confidently match to descriptions in the current taxonomic literature. Tethya spp. also appear to undergo seasonal morphological changes (M. Shaffer, personal observations from monthly monitoring for $2+$ years). For example, the same sponge's appearance may go from porous and soft to very firm over a period of a month. In addition, during the summer a sponge may be covered more extensively by crustose coralline algae (CCA), rendering it more reminiscent of T. amplexa (Bergquist and Kelly-Borges, 1991), but in the winter be completely free of CCA. Because morphology can be very variable and temporally dependent, assessing species based solely on morphology has the potential to lead to erroneous conclusions. To date, molecular differences have allowed at least one other instance of sibling species within the genus Tethya to be uncovered. Sarà et al. (1993) reported cryptic speciation in two populations of T. robusta, which were morphologically similar but genetically different. The taxonomic history, phenotypic plasticity and previous report of a species complex within the Tethya genus makes other occurrences of cryptic speciation within this group likely.

\section{Collection}

Sponges which were a priori thought to be $T$. bergquistae and $T$. burtoni were collected using SCUBA from two different locations in waters around Wellington, New Zealand (Breaker Bay: 4119'53.3"S 17449'52.6"E; Kapiti Island: 4053'23.6"S 17452'40.3"E). Additional specimens of $T$. burtoni were collected from two further locations (Matiu-Somes Island:

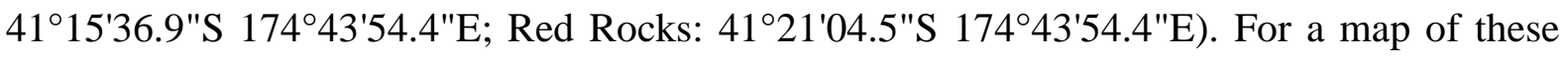
study sites, refer to Chapter 5, Figure 5.2 Sponges were collected 3-5 m apart to avoid collecting clones, at depths from 5-10m. Three sponges of unknown identity were collected from Breaker Bay $(\mathrm{n}=2)$ and Kapiti Island $(\mathrm{n}=1)$, which were texturally similar to $T$. bergquistae but the same colour as T. burtoni. A picture of each specimen was taken to aid in later identification. Tissue was extracted for both genetic and spicule analysis, and the rest of the specimen was preserved in ethanol. In total, seventy-one sponges of various morphologies were collected for phylogenetic and morphological analyses. For those sponges assumed to be 

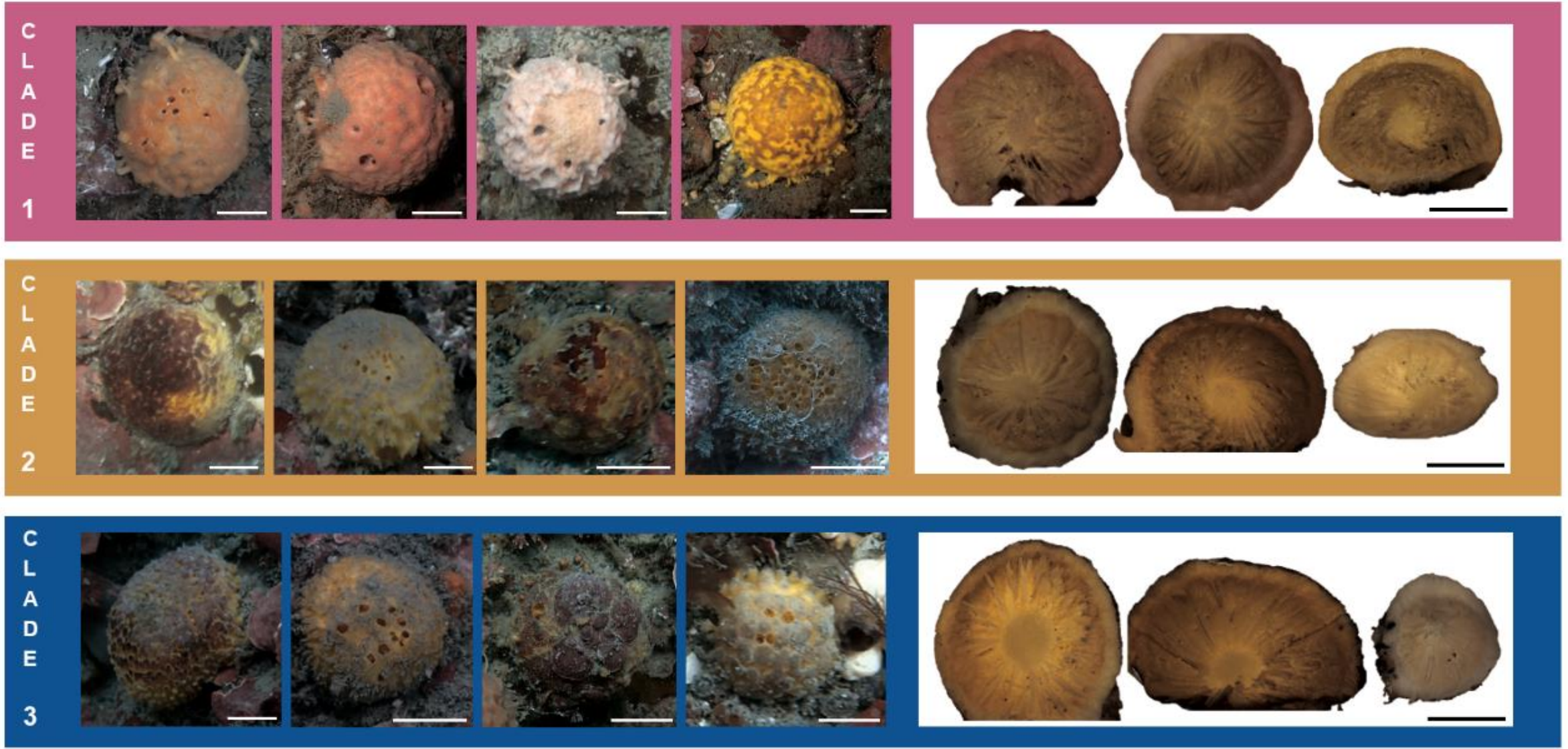

Figure 2.1. Examples of sponges collected belonging to clades identified in Figure 3, to allow comparison of macromorphological features. For each clade, cross sections that encompass the ectosome and choanosome are included for a subsample $(\mathrm{n}=3)$ of genetically identical individuals. Clade 1 (top) are examples of Tethya bergquistae, while Clades 2 and 3 (middle and bottom, respectively) are examples of $T$. burtoni. Scale bars in each photo $=10 \mathrm{~mm}$. 
T. bergquistae, samples were collected from Breaker Bay $(\mathrm{n}=10)$ and Kapiti Island $(\mathrm{n}=5)$. For those sponges assumed to be T. burtoni, samples were collected from Breaker Bay ( $\mathrm{n}=$ 17), Kapiti Island ( $n=20)$, Red Rocks $(n=5)$, Matiu-Somes Island $(n=12)$. For information on specimens collected for this study see Appendix 1, Table A1.

\section{DNA extraction, PCR amplification, and sequencing}

Tissue used for genetic analysis was taken from the inside (choanosome) of the sponge and rinsed thoroughly with distilled water to reduce the risk of contamination from epibionts or other associated organisms. DNA was extracted using a DNeasy Blood \& Tissue Kit (Qiagen), following the protocol of the manufacturer. I tried five primer sets, but two regions failed to consistently give readable sequences across all samples (ITS, primer set: ITS2.2/ITSRA2, as in Adlard and Lester (1995); and COI, primer set: dgLCO1490/dgHCO2198, as in Folmer et al. (1994)), so were discarded for further analysis. Three markers were amplified in total: 18S, $\mathrm{rnl}$ and COI-ext. 18S is a nuclear gene that codes ribosomal DNA (rDNA), and rnl is a mitochondrial partition that codes the large ribosomal subunit RNA (Boore, 1999). COI-ext includes a region downstream of COI, which is a mitochondrial coding region for the cytochrome $c$ oxidase subunit used in cellular metabolism (Folmer et al., 1994). This extended region is thought to be more informative for closely related species because its substitution pattern (mainly consisting of transversions) reveals a more progressive stage of character evolution (Erpenbeck et al., 2006a). As such, this region exhibits variation during early stages of species divergence compared to the traditionally conserved COI region (Folmer et al., 1994) that is commonly used (Erpenbeck et al., 2006a). Primer information and product sizes are shown in Table 2.1 .

Reaction and cycling conditions were similar for all three markers. Reactions were carried out in $30 \mu \mathrm{l}$ volumes consisting of: $15 \mu \mathrm{l}$ MyTaq Red Mix (Bioline), $0.6 \mu 1$ of each primer $(10 \mu \mathrm{M})$, 25 ng template DNA and a volume of distilled water to reach $30 \mu$ l. Amplification profiles were as follows: an initial denaturation at $94{ }^{\circ} \mathrm{C}$ for $5 \mathrm{~min} ; 35$ cycles of $94{ }^{\circ} \mathrm{C}$ for $30 \mathrm{~s}, \mathrm{~T}_{\mathrm{a}}$ for $55 \mathrm{~s}\left(\mathrm{~T}_{\mathrm{a}}=50{ }^{\circ} \mathrm{C}\right.$ for $18 \mathrm{~S} ; \mathrm{T}_{\mathrm{a}}=58{ }^{\circ} \mathrm{C}$ for $\mathrm{COI}$-ext and $\left.\mathrm{rnl}\right), 72{ }^{\circ} \mathrm{C}$ for $45 \mathrm{~s}$; followed by a final extension at $72{ }^{\circ} \mathrm{C}$ for $7 \mathrm{~min}$. Products were visually checked on a $1.5 \%$ agarose gel stained with RedSafe Nucleic Acid Staining Solution (20,000×) and sequenced using automated DNA sequencing services provided by Macrogen Inc. (Seoul, South Korea). Sequences were 
deposited into GenBank, with accession numbers (MH180010-MH180023) for haplotypes of all individuals provided in Appendix 1, Table A1.

Table 2.1. Primer information for markers used (18S, rnl, and COI-ext). Forward and reverse sequences presented $5^{\prime} \rightarrow 3^{\prime}$. Product sizes are in base pairs. Accession numbers refer to distinct haplotypes and genotypes that were deposited to GenBank for each marker.

\begin{tabular}{|c|c|c|c|c|}
\hline Region & Primers & $\begin{array}{l}\text { Size } \\
\text { (bp) }\end{array}$ & Ref & $\begin{array}{c}\text { Accession } \\
\text { number of } \\
\text { products }\end{array}$ \\
\hline $18 \mathrm{~S}$ & $\begin{array}{c}18 \mathrm{~S} \_1-600 \_\mathrm{F}: \\
\text { GCCAGTAGTCATATGCTTGTCTCA } \\
\text { 18S_1-600_R: } \\
\text { GACTTGCCCTCCAATTGTTC }\end{array}$ & 425 & $\begin{array}{l}\text { Knapp } \\
\text { et al. } \\
2015\end{array}$ & $\begin{array}{l}\text { MH180010 } \\
\text { MH180012 }\end{array}$ \\
\hline $\mathrm{rnl}$ & $\begin{array}{c}\text { diplo-rnl-f1: } \\
\text { TCGACTGTTTACCAAAAACATAGC } \\
\text { diplo-rnl-r1: } \\
\text { AATTCAACATCGAGGTSGGAAAC }\end{array}$ & 570 & $\begin{array}{l}\text { Lavrov } \\
\text { et al. } \\
2008\end{array}$ & $\begin{array}{r}\text { MH180013 } \\
\text { MH180019 }\end{array}$ \\
\hline $\begin{array}{c}\text { COI- } \\
\text { ext }\end{array}$ & $\begin{array}{c}\text { COX1-R1: } \\
\text { TGTTGRGGGAAAAARGTTAAATT } \\
\text { COX1-D2: } \\
\text { AATACTGCTTTTTTTGATCCTGCCGG }\end{array}$ & 425 & $\begin{array}{l}\text { Rot } \\
\text { et } a l . \\
2006\end{array}$ & $\begin{array}{l}\text { MH180020 } \\
\text { MH180023 }\end{array}$ \\
\hline
\end{tabular}

Genetic analysis

All sequences were checked for contaminants and verified as being poriferan using BLAST (http://blast.ncbi.nlm.nih.gov/Blast.cgi). Sequences were edited in Geneious 11.0.2 (Kearse $e t$ al., 2012) and aligned using CLUSTALW2.0 (Larkin et al., 2007), as implemented in Geneious 11.0.2 (Kearse et al., 2012). Phylogenetic analyses were applied to 18S, rnl and COI-ext separately, as well as to the mitochondrial partitions concatenated (COI-ext $+\mathrm{rnl})$, creating four datasets. MrModeltest v2.3 (Nylander, 2004) was used to select the best-fitting model of nucleotide substitution for each dataset under the Akaike information criterion (AIC), and it was determined that the SYM+G model was the best fitting model for all datasets. The closely related $T$. actinia was chosen as an outgroup because both the $18 \mathrm{~S}$ region and its complete mitochondrial genome have been sequenced and made available on GenBank, making it an ideal candidate to root all three markers (accession numbers: AY320033 for rnl and COI-ext; AY878079 for 18S). 
Phylogenetic trees were constructed using both Maximum Likelihood (ML) and Bayesian Inference $(\mathrm{BI})$ methods for each data set. ML constructions were performed in PAUP* 4.0 (Swofford, 2002) and BI constructions were performed in MrBayes 3.2.6 (Ronquist et al., 2012), using the model criterion generated from MrModeltest v2.3 (Nylander, 2004). For ML analyses, a bootstrap analysis using a tree-bisection-reconnection (TBR) heuristic search with 1,000 replicates was performed to determine topological confidence of the tree. For BI analyses, posterior probability of trees was estimated using Monte Carlo Markov Chain (MCMC) analyses, where one million generations were run, sampling four chains every 100 generations, with a burn-in of 0.25 . For each marker, p-distances were calculated within and between clade as a measure of divergence using MEGA 7 (Kumar et al., 2015). In addition, pdistances were calculated between and within T. bergquistae (Clade 1) and T. burtoni (Clades $2+3$ combined).

\section{Morphological analysis}

To determine if morphological characteristics were consistent with the observed genetic clades, a subsample of six sponges from each clade was selected for spicule analysis. I chose genetically identical individuals that fell consistently into the same clade for all three markers. Samples were collected from the same location and at the same time in order to reduce potential environmental and seasonal variation in spicule morphology (Bell et al., 2002). Each sponge was cut in half to photograph its cross section. Further, a small piece of tissue was embedded in paraffin wax and sectioned to $20 \mu \mathrm{m}$ using a rotary microtome (Leica Biosystems RM2235) to examine the location, arrangement and density of spicules across the ectosome and choanosome. A $\sim 5 \mathrm{~mm}^{3}$ tissue sample was then removed from each specimen and dissolved in bleach at $50^{\circ} \mathrm{C}$ for $48 \mathrm{~h}$ and the remaining spicules were then washed with distilled water and rinsed 3x with absolute ethanol, following the recommendations of Hooper (2003). One millilitre of the remaining spicule solution was mounted onto a slide using DPX and examined at a magnification of 100-200×, using a compound light microscope (Leica Microsystems DM LB) with attached digital camera (Canon EOS 70D). Spicule type (see Appendix 2 for definitions of spicule types) and location (defined as region of tissue in the sponge - cortex versus choanosome) was recorded for a qualitative analysis. For a quantitative comparison, the sizes of 30 of each spicule type which were present in all sponges (strongyloxea, megasters, micrasters) were recorded per sponge. For megascleres (strongyloxea), the length and width 
were recorded for both thicker and thinner auxiliary types (stronglyoxea and styles, respectively). For both megasters and micrasters, the diameter and $\mathrm{R}: \mathrm{C}$ ratio (ray length to centre length ratio) were recorded, following Bergquist and Kelly-Borges (1991) and Sàra and Sàra (2004). Size measurements for all megasters (spherasters and oxyspherasters) were grouped, as well as micrasters (oxyasters, chiasters, stronglyasters and tylasters), because they could not be differentiated using light microscopy. Sizes were measured using ImageJ (Schneider et al., 2012). Non-parametric multidimensional scaling (nMDS) ordinations were generated using Euclidean distance on Primer v7 (Clarke, 1993) to determine any clustering based on average, minimum and maximum sizes. Spicules from all 18 sponges, which were subsampled for spicule analysis, were also observed using Scanning Electron Microscopy (SEM) to visualise the megasters and micrasters at higher resolution and magnification. Spicules preserved in ethanol were mounted on stubs, air dried and coated with a gold/palladium alloy using a sputter coater. Scanning electron micrographs were taken with the Hitachi TM3000 Benchtop Scanning Electron Microscope in high vacuum mode.

\section{Results}

\section{Molecular phylogeny}

In total, $1420 \mathrm{bp}$ (425 bp for $18 \mathrm{~S}, 570 \mathrm{bp}$ for rnl, $425 \mathrm{bp}$ for COI-ext) were sequenced for 71 Tethya individuals. For all trees, branch confidence (via bootstrap/posterior probability values) ranged from 64-100\%/0.91-1 for divergences between the main clades (Figures 2.2 and 2.3). $18 \mathrm{~S}$ resolved the clades above 86\%/0.96 and rnl above 74\%/0.99, but COI-ext exhibited slightly lower confidence from $63 \% / 0.91$. The nuclear $18 \mathrm{~S}$ region grouped sponges into three genotypes, and for the mitochondrial rnl and COI-ext partitions there were seven and four haplotypes present, respectively. The concatenated dataset for the mitochondrial markers revealed 15 different haplotypes (Figure 2.3). For each individual locus, phylogenetic reconstructions revealed three divergent clades that each contained samples from all sampling locations; in other words, none of the clades contained individuals from strictly one location. For $\mathrm{rnl}$ and $18 \mathrm{~S}$, those sponges that were thought to be $T$. bergquistae all grouped together into one clade (Clade 1), but for COI-ext, one individual that was thought to be $T$. bergquistae grouped with Clade 2 (T. burtoni clade). For T. burtoni, rnl and $18 \mathrm{~S}$ generated congruent placement of sponges into two separate groups. However, COI-ext placed sponges differently, where some sponges that belonged to Clade 2 for rnl and $18 \mathrm{~S}$ were placed into Clade 3 ; and in 
addition, two sponges which were thought to be $T$. burtoni were placed into Clade 1 ( $T$. bergquistae clade). The three uncertain yellow sponges (Figure 2.1) grouped with $T$. bergquistae for all three markers.

Divergence was calculated between and within all genetic clades using p-distances (Table 2.2). Intraclade $\mathrm{p}$-distance across all three markers ranged from $0-0.13 \%$ (average $=0.04 \%$, stdev $=$ $0.06 \%$ ), whereas interclade p-distances for all pairwise clade comparisons across all markers ranged from $0.5-11.2 \%$ (average $=5.29 \%$, stdev $=3.83 \%$ ). For all pairwise clade comparisons, interclade p-distances for $18 \mathrm{~S}(0.5-1.2 \%)$ were lower than for the mt-markers rnl and COI-ext (4.8-8.6\%, 6.4-11.2\%, respectively). For T. bergquistae (Clade 1), the intraclade p-distance for all markers was zero. For T. burtoni (Clades $2+3$, combined), intraclade p-distances were: zero for $18 \mathrm{~S}, 2.1 \%$ for $\mathrm{rnl}$, and $4.2 \%$ for COI-ext. The divergence between $T$. bergquistae and T. burtoni was: $0.8 \%$ for $18 \mathrm{~S}, 6.6 \%$ for $\mathrm{rnl}$, and $9.0 \%$ for COI-ext.

Table 2.2. Genetic divergence (p-distances, \%) for Tethya spp. for all gene markers. Intraclade p-distances are reported for individuals within clades, while interclade values are pairwise comparisons between all clades.

\begin{tabular}{|c|c|c|c|c|c|}
\hline \multicolumn{6}{|l|}{$18 S$} \\
\hline \multicolumn{2}{|c|}{ Intraclade } & \multicolumn{4}{|c|}{ Interclade } \\
\hline & & & Clade 1 & Clade 2 & Clade 3 \\
\hline Clade 1 & 0 & Clade 1 & 0 & & \\
\hline Clade 2 & 0 & Clade 2 & $0.7 \%$ & 0 & \\
\hline Clade 3 & 0 & Clade 3 & $1.2 \%$ & $0.5 \%$ & 0 \\
\hline \multicolumn{6}{|l|}{ rnl } \\
\hline \multicolumn{2}{|c|}{ Intraclade } & \multicolumn{4}{|c|}{ Interclade } \\
\hline Clade 1 & 0 & Clade 1 & Clade 1 & & Clade 3 \\
\hline Clade 2 & 0 & Clade 2 & $5.9 \%$ & 0 & \\
\hline Clade 3 & $0.13 \%$ & Clade 3 & $8.6 \%$ & $4.8 \%$ & 0 \\
\hline \multicolumn{6}{|c|}{ COI-ext } \\
\hline \multicolumn{2}{|c|}{ Intraclade } & \multicolumn{4}{|c|}{ Interclade } \\
\hline & & & Clade 1 & Clade 2 & Clade 3 \\
\hline Clade 1 & 0 & Clade 1 & 0 & & \\
\hline Clade 2 & $0.10 \%$ & Clade 2 & $6.4 \%$ & 0 & \\
\hline Clade 3 & $0.13 \%$ & Clade 3 & $11.2 \%$ & $8.3 \%$ & 0 \\
\hline
\end{tabular}




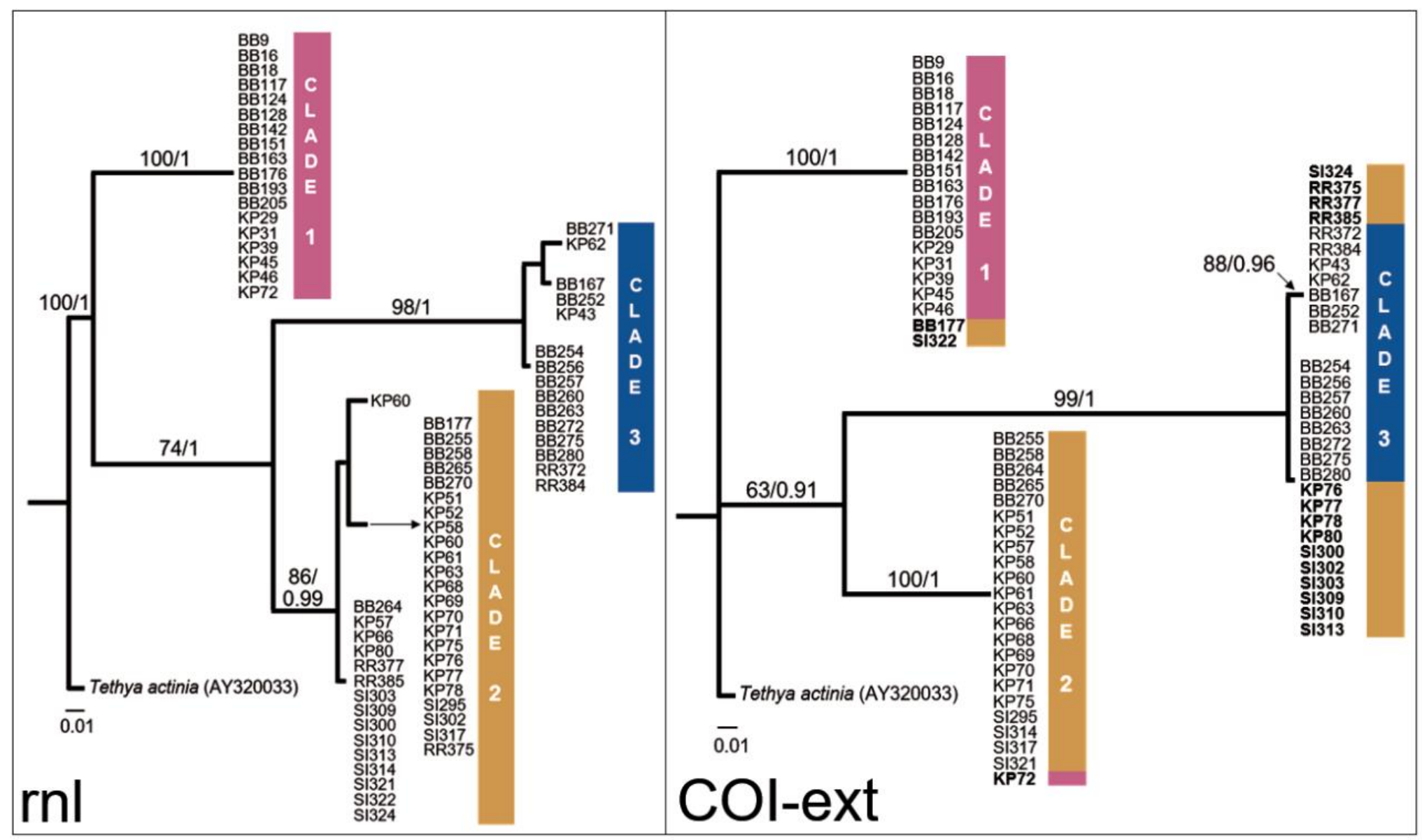

Figure 2.2. Phylograms showing the relationship between Tethya bergquistae (Clade 1) and T. burtoni (Clades 2 and 3) based on individual mitochondrial markers ( $r n l$ and COI-ext). Topology generated from Maximum Likelihood (ML) analysis. ML bootstrap confidence values/Bayesian Inference (BI) posterior probabilities given on each branch. Tethya actinia was selected as an outgroup. Inconsistencies in the COI-ext tree versus rnl are highlighted by having sponges retain the colour of the rnl placement and by bolding individuals. Scale bar $=$ substitutions per site. 


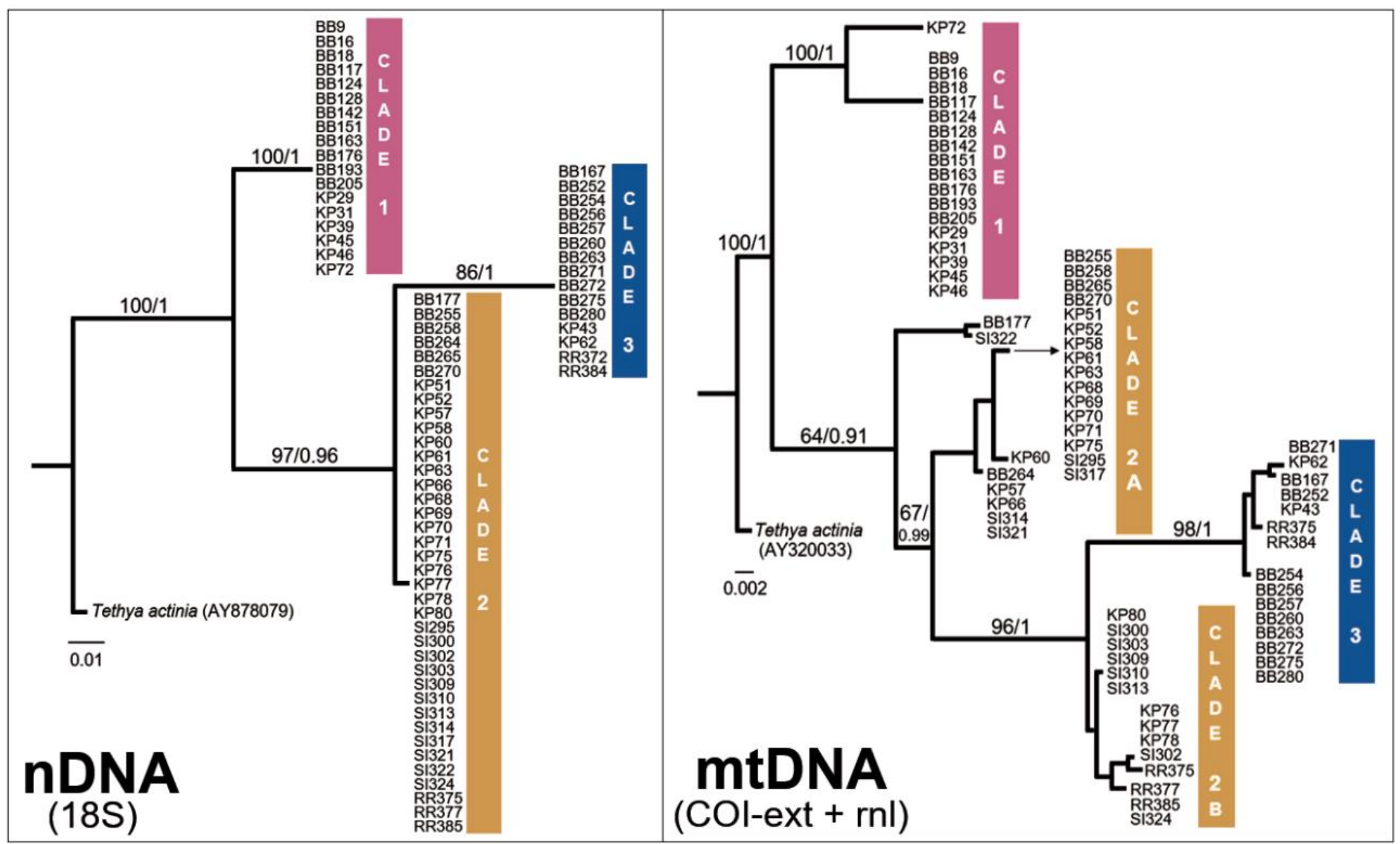

Figure 2.3. Phylograms showing the relationship between Tethya bergquistae (Clade 1) and T. burtoni (Clades $2 \mathrm{~A}+\mathrm{B}$ and 3) based on nuclear DNA (18S) and mitochondrial DNA (concatenated COI-ext + rnl). Topology generated from Maximum Likelihood (ML) analysis. ML bootstrap confidence values/Bayesian Inference (BI) posterior probabilities given on each branch. T. actinia was selected as an outgroup. Scale bar = substitutions per site. 


\section{Morphological analysis}

All sponges contained megascleres that were strongyloxea and anisostronglyes, along with smaller, thinner auxiliary styles. Megasters were spherasters and oxyspherasters, and micrasters were tylasters, chiasters, stronglyasters and oxyasters. Spicule sizes are summarized in Table 3. Average strongyloxea and auxiliary style lengths $\times$ widths were $1061 \pm 275 \mu \mathrm{m} \times$ $21 \pm 6 \mu \mathrm{m}$ and $399 \pm 116 \mu \mathrm{m} \times 13 \pm 4 \mu \mathrm{m}$, respectively. Respective megaster and micraster diameters were $44 \pm 10 \mu \mathrm{m}$ and $14 \pm 3 \mu \mathrm{m}$, and the average $\mathrm{R}: \mathrm{C}$ ratio was $\sim 0.8 \pm 0.2$ for all asters. n-MDS plots for spicule size (average, minimum and maximum sizes) revealed no evident clustering (Figure 2.4). Qualitative differences were observed between the location and density of megasters within the different genetic clades. Those sponges belonging to Clade 1 contained megasters throughout both the cortex and choanosome, whereas those sponges in Clades 2 and 3 had megasters in the cortex, but little to none in the choanosome. Within the $T$. burtoni clades (Clades 2 and 3), there were no differences in spicule composition detectable by light microscopy.

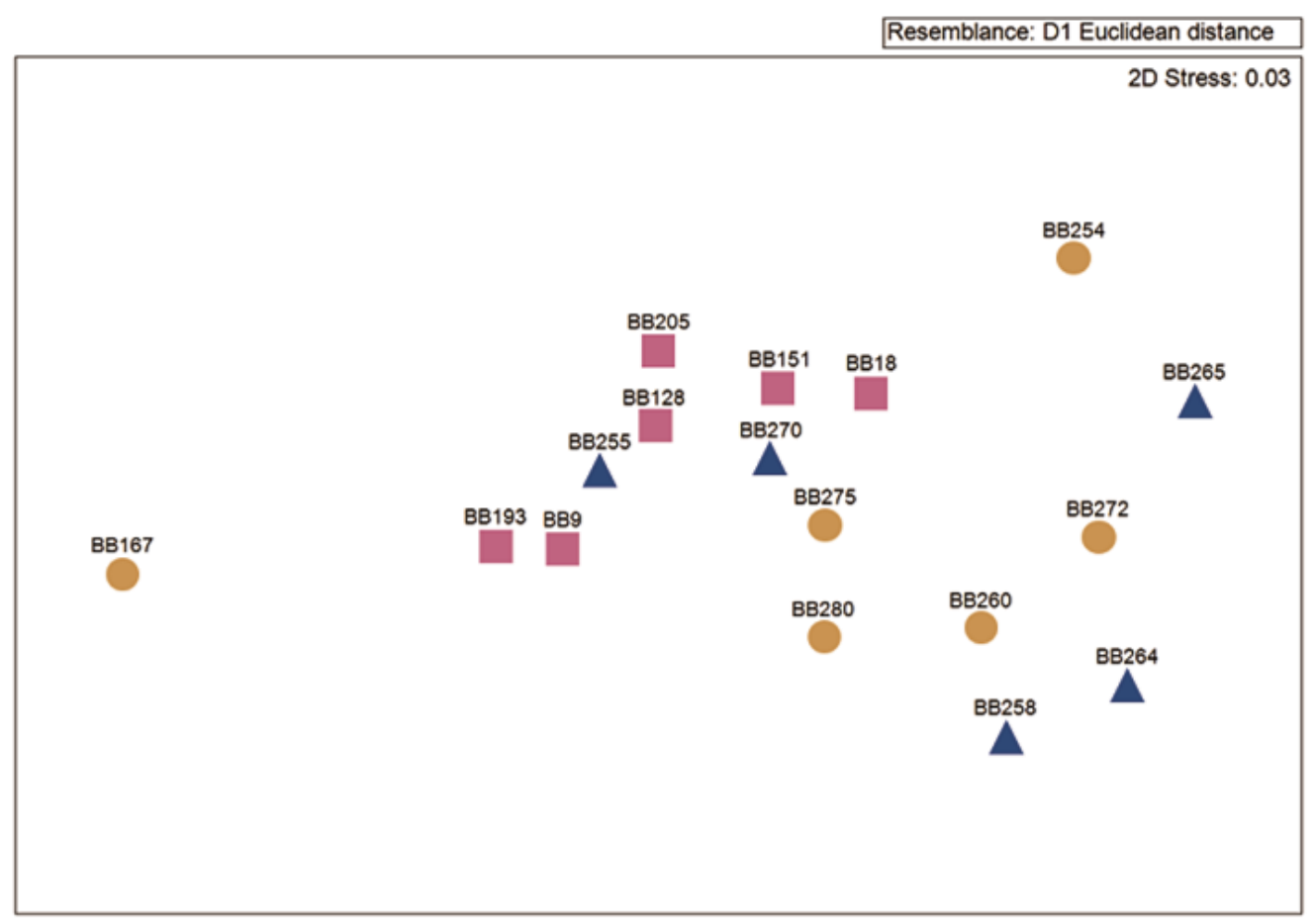

Figure 2.4. Nonparametric-multidimensional (nMDS) scaling ordination of spicule sizes for Tethya bergquistae (pink squares, Clade 1) and T. burtoni (orange circles, Clade 2; and blue triangles, Clade 3). Clades are identified in Figures 3. Spicules sizes are based on measurements from strongyloxea, megasters and micrasters $(n=30$ per sponge $)$. Codes above each sample refer to individual sample ID (refer to Appendix 1 for more information on individual samples). A subset of all samples is presented in the nMDS, based on a Euclidean distance matrix. 
Scanning electron micrographs of spicules from the three genetic clades also revealed no visible differences in megastar or micraster morphology (Figure 2.5). However, sponges belonging to the $T$. bergquistae clade contained an additional spined oxyaster, which was absent in the T. burtoni clades. A qualitative assessment of cross sections from this subsample of sponges revealed high plasticity within each genetic clade, and no clear differences between clades (Figure 2.1). Further, $20 \mu \mathrm{m}$ cross sections examining the spicule composition between the choanosome and ectosome revealed that $T$. bergquistae contained more asters in the choanosome than T. burtoni (Figure 2.6). However, within T. burtoni, there were no evident differences between Clades 2 and 3. While some members of Clade 2 appeared to contain a slightly higher density of asters in the cortex, this was not consistent for all individuals within that clade.

Table 2.3. Mean lengths for spicules in Tethya bergquistae (Clade 1) and T. burtoni (Clades 2 +3 ) for three genetic clades identified by $18 \mathrm{~S}$ and rnl (Figures 2 and 3) and for all samples combined (overall). Strongyloxea are presented as length $\times$ width. R:C refers to ray length:diameter-to-centre ratios. All sizes are in microns $(\mu \mathrm{m})$.

\begin{tabular}{|c|c|c|c|c|c|c|}
\hline & \multicolumn{2}{|c|}{ Megascleres } & \multicolumn{2}{|c|}{ Megasters } & \multicolumn{2}{|c|}{ Micrasters } \\
\hline & $\begin{array}{l}\text { Stronglyoxea } \\
\quad(1 \times w)\end{array}$ & $\begin{array}{c}\text { Style } \\
(\mathbf{l} \times \mathbf{w})\end{array}$ & d & $\mathbf{R : C}$ & d & $\mathbf{R}: \mathbf{C}$ \\
\hline $\begin{array}{c}\text { Clade } \\
1\end{array}$ & $\begin{array}{c}1032.0 \pm 219.4 \\
\times \\
22.7 \pm 5.7\end{array}$ & $\begin{array}{c}356.4 \pm 103.6 \\
\times \\
14.4 \pm 3.3\end{array}$ & $\begin{array}{c}42.9 \\
\pm \\
12.5\end{array}$ & $\begin{array}{c}0.75 \\
\pm \\
0.15\end{array}$ & $\begin{array}{c}13.7 \\
\pm \\
3.2\end{array}$ & $\begin{array}{c}0.81 \\
\pm \\
0.19\end{array}$ \\
\hline $\begin{array}{c}\text { Clade } \\
2\end{array}$ & $\begin{array}{c}1054.7 \pm 320.4 \\
\times \\
21.6 \pm 4.6\end{array}$ & $\begin{array}{c}424.1 \pm 108.1 \\
\times \\
13.6 \pm 3.8\end{array}$ & $\begin{array}{c}44.7 \\
\pm \\
6.4\end{array}$ & $\begin{array}{c}0.73 \\
\pm \\
0.15\end{array}$ & $\begin{array}{c}15.2 \\
\pm \\
1.4\end{array}$ & $\begin{array}{c}0.79 \\
\pm \\
0.16\end{array}$ \\
\hline $\begin{array}{c}\text { Clade } \\
3\end{array}$ & $\begin{array}{c}1104.0 \pm 273.5 \\
\times \\
16.7 \pm 5.3\end{array}$ & $\begin{array}{c}419.7 \pm 124.7 \\
\times \\
9.9 \pm 2.9\end{array}$ & $\begin{array}{c}44.1 \\
\pm \\
9.0\end{array}$ & $\begin{array}{c}0.86 \\
\pm \\
0.15\end{array}$ & $\begin{array}{c}12.4 \\
\pm \\
2.0\end{array}$ & $\begin{array}{c}1.07 \\
\pm \\
0.25\end{array}$ \\
\hline Overall & $\begin{array}{c}1061.2 \pm 275.3 \\
\times \\
20.6 \pm 5.8\end{array}$ & $\begin{array}{c}399.0 \pm 115.9 \\
\times \\
12.8 \pm 3.9\end{array}$ & $\begin{array}{c}43.9 \\
\pm \\
9.7\end{array}$ & $\begin{array}{c}0.77 \\
\pm \\
0.16\end{array}$ & $\begin{array}{c}13.8 \\
\pm \\
2.6\end{array}$ & $\begin{array}{c}0.88 \\
\pm \\
0.23\end{array}$ \\
\hline
\end{tabular}



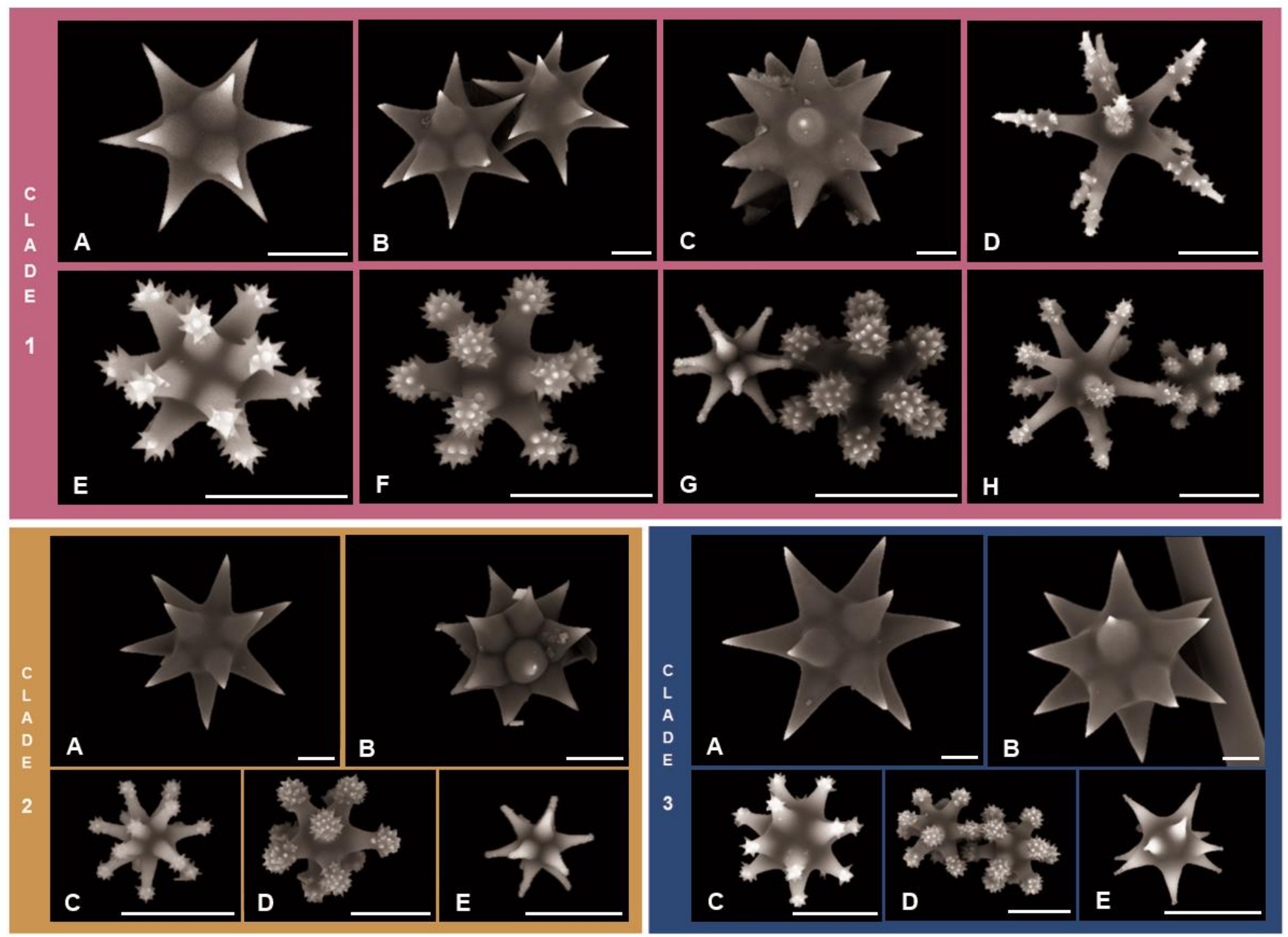

Figure 2.5. Scanning electron micrographs of spicules belonging to Tethya bergquistae (Clade 1) and Tethya burtoni (Clade $2+3$ ), from sponges belonging to the three genetic clades of Tethya identified in Figure 3. Clade 1: A-C = spherasters; $\mathrm{D}=$ spined oxyaster; $\mathrm{E}-\mathrm{H}=$ tylasters; $\mathrm{G}=$ oxyaster. Clade 2 and $3: \mathrm{A}-\mathrm{B}=$ spherasters; $\mathrm{C}-\mathrm{D}=$ tylasters; $\mathrm{E}$ $=$ oxyaster. Scale bars are located in the bottom right of each micrograph and represent $10 \mu \mathrm{m}$. 

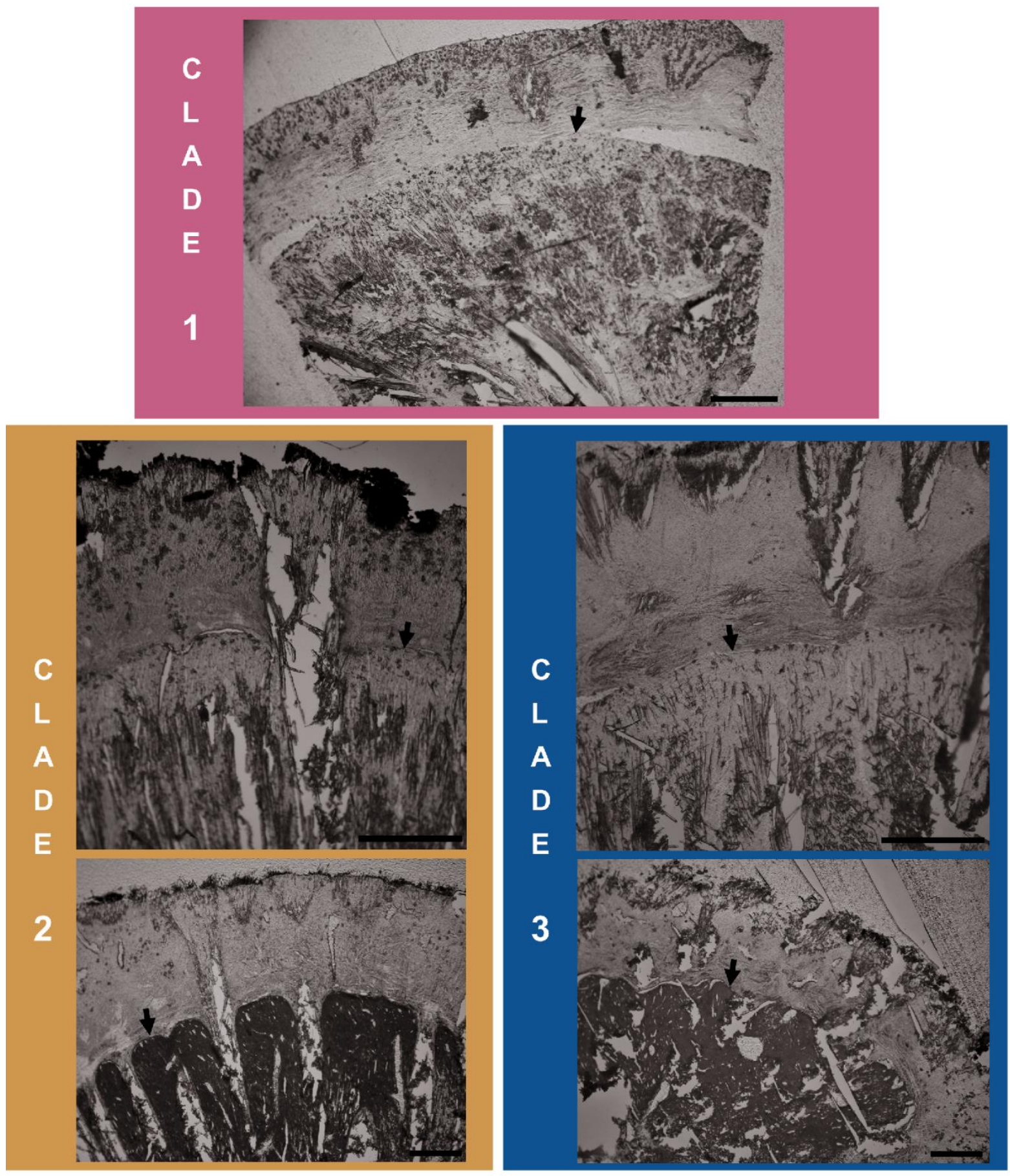

Figure 2.6. Cross sections from small pieces of tissue (20 $\mu \mathrm{m}$ thick) from Tethya spp., where Clade 1 is T. bergquistae and Clades 2 and 3 are T. burtoni. Arrows are pointing to the line that divides the outer cortex and the inner choanosomal tissue. Black scale bars represent $500 \mu \mathrm{m}$. 


\section{Discussion}

Here, I document discordance between molecular and morphological descriptions of Tethya spp. in central New Zealand and highlight the potential for cryptic speciation to go undetected based on morphological features alone. Phylogenetic reconstructions based on 18S, rnl and COI-ext revealed three genetic clades, with a speciation event likely occurring in the group described as T. burtoni. Morphological analyses based on spicule size and composition allowed T. bergquistae to be differentiated from T. burtoni, but failed to reveal differences between the T. burtoni clades. To my knowledge, this is only the second report of cryptic species on the basis of molecular differences within the group Tethya (with the first being T. robusta, by Sarà et al. (1993)). Understanding how organisms evolve is central to ecology and evolution, but can be particularly complex for phenotypically similar organisms living in sympatry with no obvious barriers to reproduction, reflected in Tethya spp. examined here. Overall, my study highlights the need to use more than one method to define sponge species boundaries in a reliable way.

\section{Species delineation based on genetics}

The phylogenetic relationship of Tethya spp. was not congruent for all three markers, as COIext placed sponges into different clades with most discrepancies occurring in the T. burtoni group. The use of COI to delineate species has had differential success among sponges (Erpenbeck et al., 2006b; Wörheide and Erpenbeck, 2007; Erpenbeck et al., 2007; Poppe et al., 2010; Belinky et al., 2012), as well as other organisms (Shearer and Coffroth, 2008; Derycke et al., 2010), resulting in debate around its use as an informative marker (Waugh, 2007). The COI region often has low variability (Bucklin et al., 2011), and this conservativeness can fail to capture evolutionary relationships between closely related species. I instead employed COIext to avoid this drawback, as this region downstream of COI is substitutes earlier during species divergence (Erpenbeck et al., 2006a). While variation between T. bergquistae and $T$. burtoni, as well as within T. burtoni, was captured, the relationship produced from COI-ext was incongruent with that produced from $18 \mathrm{~S}$ and $\mathrm{rnl}$. For species living in sympatry, introgression can be common, resulting in semipermeable species boundaries (Rüber et al., 2001, Harrison and Larson, 2014). Genes can introgress at different rates (called differential introgression), which results in phylogenies from multiple markers that are in disagreement (Harrison and Larson, 2014). It is possible that the inconsistency in the COI-ext phylogeny is 
a product of this, and if true, gives evidence of introgression between $T$. bergquistae and $T$. burtoni. For instance, one sponge (KP72) that was thought to be T. bergquistae (as evidenced by its placement into Clade 1 for $18 \mathrm{~S}$ and $\mathrm{rnl}$ ) was placed into the T. burtoni clade (Clade 2) for COI-ext. In addition, two sponges (BB177 and SI322), which were thought to be T. burtoni (from their placement into Clade 2 by $18 \mathrm{~S}$ and $\mathrm{rnl}$ ), were placed into the $T$. bergquistae clade (Clade 1) for COI-ext. Within T. burtoni, there is more disagreement between COI-ext and the other markers within the clades. For example, those sponges belonging to Clade 2B of the mitochondrial concatenated dataset (Figure 2.3) were placed into Clade 2 by $18 \mathrm{~S}$ and rnl, but Clade 3 for COI-ext. It is possible that this group of sponges, which possess genes common to two different putative species (discussed below), have also undergone introgressive hybridization. Some corals have also been found to exhibit high degrees of sympatry, cryptic speciation and introgression (Ladner and Palumbi, 2012), and perhaps shared life-history traits between corals and sponges (i.e., sessile, sexual and asexual reproductive ecology) shape the evolutionary history of these populations. While additional sequencing work (particularly using diploid markers) would provide clarity on this topic, it appears highly probable that the species boundaries between $T$. bergquistae and T. burtoni, as well as between the putative $T$. burtoni cryptic species, are actually semipermeable.

The definition of a species continues to be debated, and when complex evolutionary processes like introgression can shape species, understanding species delineations becomes more complicated. The topology produced from $18 \mathrm{~S}$ clearly differentiates the clades in this study. $18 \mathrm{~S}$ is a slowly evolving gene region (Berntson, 2001), and as such often captures interspecific variation rather than intraspecific variation. Three separate clades, despite the slow evolving nature of this gene, provide strong evidence of three different species. Most interestingly, two clades exist within $T$. burtoni and suggest cryptic speciation within the group. Other measures exist (e.g., p-distances) to more definitively set a quantitative value to define species divisions. Comparing the p-distances obtained in this study to other observed divergence values for sponges (Table 2.4), both within- and between-clade distances give further evidence for species-level relatedness. The intraclade p-distances here were about $\sim 5-50$ times less than the interclade values for all pairwise comparisons of clades, which is consistent with intraspecific values for other sponge taxa. These intraclade distances suggest that sponges belonging to the same clade are conspecific. When comparing the individual markers used here to the same markers (or similarly evolving markers) in other studies, the interclade p-distances calculated 
for all pairwise clade comparisons in this study compare to interclade p-distances for other sponge species (Table 2.4). This further reveals that clades do in fact correspond to species. For example, Scopalina lophyropoda was reported as being comprised of a cryptic species complex (Blanquer and Uriz, 2007), and the interclade p-distances for 18S (0.3-2\%) were on the same scale as for those clades I found within T. burtoni ( $0.5 \%)$. Considering $T$. bergquistae (Clade 1) versus $T$. burtoni (Clades $2+3$, combined), divergence between the two species was: $0.8 \%$ for $18 \mathrm{~S}, 6.6 \%$ for $\mathrm{rnl}$ and $9 \%$ for COI-ext. Divergence estimates between the T. burtoni clades (Clade 2 versus Clade 3 ) were on the same order: $0.5 \%$ for $18 \mathrm{~S}, 4.8 \%$ for $\mathrm{rnl}$ and $8.3 \%$ for COI-ext. Divergence within T. burtoni that is on the same scale as between T. burtoni and $T$. bergquistae gives further evidence that the two $T$. burtoni clades correspond to different species. Interestingly, differentiation between both $T$. burtoni clades was not a product of location (population structure of T. burtoni discussed in Chapter 5). Instead, sponges that lived sympatrically were strongly genetically different, suggesting reproductive isolation for sponges in the same location. Cryptic speciation has been recorded throughout the Porifera phylum (Solé-Cava et al., 1991; Muricy et al., 1996; Nichols and Barnes, 2005; Wörheide et al., 2008; Xavier et al., 2010; DeBiasse and Hellberg, 2015; Knapp et al., 2015; Uriz et al., 2017), and is also likely for what is known as T. burtoni. This genetic data set combined gives strong evidence that $T$. burtoni is in fact comprised of a species complex, being more genetically diverse than previously thought based on morphology alone.

\section{Discordance between molecular and morphological characteristics}

My analysis of spicule size/type, in conjunction with the qualitative assessment of macromorphological features (colour and cross sections), was inconsistent with the molecular phylogeny that was generated. Macromorphological features allowed sponges belonging to the T. bergquistae clade to be distinguished from sponges belonging to the T. burtoni clades; however, I was not able to identify sponges belonging to separate genetic clades within $T$. burtoni from morphology alone. The three uncertain, bright yellow specimens grouped together with those sponges belonging to $T$. bergquistae, which has not previously been described as yellow (Bergquist and Kelly-Borges, 1991; Hooper and Wiedenmayer, 1994; Battershill et al., 2010), indicating that colour is not a reliable distinguishing feature in this case. Qualitative observations revealed no obvious differences in cross sections between the T. burtoni clades, and instead were highly variable within each clade. While generally cross sections are a key 
Table 2.4. Summary of genetic divergence (p-distances, \%) for various sponge species. Ranges given for intraclade and interclade p-distances.

\begin{tabular}{|c|c|c|c|c|}
\hline Species & Marker & $\begin{array}{l}\text { Intraclade } \\
\text { p-distance }\end{array}$ & $\begin{array}{l}\text { Interclade } \\
\text { p-distance }\end{array}$ & Reference \\
\hline \multirow{2}{*}{ Aplysina spp. } & $\mathrm{COI}$ & 0 & - & \multirow{2}{*}{$\begin{array}{c}\text { Cruz-Barraza et } \\
\text { al., } 2012\end{array}$} \\
\hline & ITS1-5.8-ITS2 & 0 & $0.1-2.2$ & \\
\hline Astrosclera willeyana & $\mathrm{COI}$ & - & $0.2-0.4$ & Wörheide, 2006 \\
\hline \multirow{2}{*}{ Cliona spp. } & $\mathrm{COI}$ & - & $2.7-8.7$ & \multirow{4}{*}{$\begin{array}{c}\text { De Paula et al., } \\
2012\end{array}$} \\
\hline & ITS & - & $5.9-14.8$ & \\
\hline \multirow{2}{*}{ Cliona celata* } & $\mathrm{COI}$ & - & $2.7-8.3$ & \\
\hline & ITS & - & $8.1-11.6$ & \\
\hline \multirow{3}{*}{ Cliona celata* } & $\mathrm{COI}$ & $0.1-0.5$ & $6.2-8.4$ & \multirow{3}{*}{$\begin{array}{l}\text { Xavier et al., } \\
2010\end{array}$} \\
\hline & Atp8 & $0-0.5$ & $7.9-16$ & \\
\hline & $28 \mathrm{~S}$ & 0 & $2.8-5.4$ & \\
\hline \multirow{2}{*}{ Haliclona sp. } & ITS1 & $1.7-3.0$ & - & \multirow{2}{*}{$\begin{array}{l}\text { Redmond and } \\
\text { McCormack, } \\
2009\end{array}$} \\
\hline & ITS2 & $0.7-1.8$ & - & \\
\hline \multirow{3}{*}{ Hemimycale spp. } & $\mathrm{COI}$ & - & $8.4-8.7$ & \multirow{3}{*}{ Uriz et al., 2017} \\
\hline & $28 \mathrm{~S}$ & - & $1.8-2.2$ & \\
\hline & $18 \mathrm{~S}$ & - & $1.4-1.9$ & \\
\hline \multirow{2}{*}{ Hexadella spp. } & $\mathrm{COI}$ & $0-3.5$ & $3.9-8.7$ & \multirow{2}{*}{$\begin{array}{c}\text { Reveillaud et al., } \\
2010\end{array}$} \\
\hline & ATPS & $0-6.3$ & $10-28.5$ & \\
\hline Ianthella basta* & $\mathrm{COI}$ & - & $0.5-1.75$ & $\begin{array}{c}\text { Andreakis et al., } \\
2012\end{array}$ \\
\hline \multirow{2}{*}{ Plocamionida spp. } & $\mathrm{COI}$ & 0.5 & $1.3-20$ & \multirow{2}{*}{$\begin{array}{l}\text { Reveillaud et al., } \\
2011\end{array}$} \\
\hline & $28 \mathrm{~S}$ & - & $0.1-3.3$ & \\
\hline \multirow{3}{*}{$\begin{array}{c}\text { Scopalina } \\
\text { lophyropoda* }\end{array}$} & $\mathrm{COI}$ & $0-3$ & $13-22$ & \multirow{3}{*}{$\begin{array}{c}\text { Blanquer and } \\
\text { Uriz, } 2007\end{array}$} \\
\hline & $28 \mathrm{~S}$ & $0-0.8$ & $2-19$ & \\
\hline & $18 \mathrm{~S}$ & 0.1 & $0.3-2$ & \\
\hline \multirow{3}{*}{ Tethya spp. } & COI-ext & $0-0.1$ & $6.4-11.2$ & \multirow{6}{*}{ This study } \\
\hline & rnl & $0-0.1$ & $4.8-8.6$ & \\
\hline & $18 \mathrm{~S}$ & 0 & $0.5-1.2$ & \\
\hline \multirow{3}{*}{ Tethya burtoni* } & COI-ext & 0.1 & 8.3 & \\
\hline & rnl & $0-0.1$ & 4.8 & \\
\hline & $18 \mathrm{~S}$ & 0 & 0.5 & \\
\hline
\end{tabular}

*reported as cryptic species 
defining feature between species of Tethya, they did not provide clear resolution here. There was a marked difference between the spicule composition of $T$. bergquistae and the T. burtoni complex (more asters in the choanosome of the former), but there were no differences evident within the T. burtoni clades. Morphological characteristics therefore provided no clear evidence of speciation within T. burtoni. Because there is a lack of defining characteristics available for Porifera, it is common for genetic diversity (and cryptic speciation) to go undetected, where molecular and morphological relationships are commonly incongruent (McCormack et al., 2002; Erpenbeck et al., 2006b; Xavier et al., 2010). Determining which morphological traits are informative in species differentiation can also be complicated. For example, Erpenbeck et al. (2006b) documented that sponges containing highly divergent skeletal features are actually closely genetically related. In contrast, spicules within the same species may be highly variable due to environmental influences (Bell et al., 2002). I show here that morphological features may not be able to reveal species boundaries, as perhaps skeletal changes following divergence may be delayed. Mutations may accumulate without altering the phenotype when nucleotide changes have no effect on how its product (protein) folds and its function overall (Avise, 2012), which could explain delayed morphological expression in genetically different individuals. While it is possible that subtle morphological differences may exist, they were not able to be detected in this study. The high phenotypic plasticity observed across individuals of $T$. burtoni resulted in a lack of clear diagnostic features for sponges belonging the different genetic clades. Furthermore, the potential introgression events between cryptic species may make detecting morphological differences between such species more difficult. To avoid this potential confounding factor, I used sponges which showed no introgression based on our markers for the morphological analysis presented here. However, I cannot confirm that these sponges have not undergone introgression, as I only surveyed three genes. A survey of other regions of the genome (specifically using diploid markers) may reveal clearer relationships and show the scope of introgressive hybridization between the T. burtoni cryptic species. This may shed more light on differences between plasticity versus distinct morphological features that allow species to be characterized. With such incongruence between morphological and molecular data, it is imperative to use a combination of methods to correctly delineate species in order to fully understand the species evolutionary relationships. 


\section{Conclusion}

This chapter highlights the complexity in delineating sympatric, morphologically similar species when disagreement between morphological and molecular traits exist, and the importance of using multiple taxonomic methods. While traditional morphology-based taxonomy can be the first step in species identification, I show that it can fail to reveal cryptic species. Being able to confidently identify a species is crucial to any ecological or evolutionary study, yet remains a challenge. Organisms with few defining traits, like sponges, present an opportunity to examine potential morphological and molecular discord, and highlight the complexity in defining species boundaries. Failure to detect cryptic species may result in an underestimation of biodiversity and an incorrect interpretation of functional roles of an organism. In addition, it can mislead our understanding of connectivity patterns and evolutionary relationships, if interspecific diversity is confused for intraspecific variation. As such, I strongly recommend the use of integrative taxonomy in species identification. 
CHAPTER 3:

SEASONAL SEXUAL REPRODUCTION IN TETHYA SPP. ON NEW ZEALAND TEMPERATE REEFS 



\section{Introduction}

The environment plays a critical role in regulating reproduction for many marine invertebrates (Yamahira, 2004; Naylor, 2013). Future shifts in climate are predicted to cause increases in water temperature, ocean acidification and storm intensity/frequency over the next century (IPCC, 2014) and beyond, which will likely have severe consequences for organisms that use environmental cues during reproduction (Visser et al., 2004; Przeslawski et al., 2008). Temperature changes have been shown to trigger gametogenesis in organisms including corals, marine worms, scallops, sponges, and urchins (Beauchamp, 1992; Sastry, 1966; Olive et al., 1998; Ettinger-Epstein et al., 2007; Epherra et al., 2015); and lunar cycles have also been linked to spawning activities (Fromont and Bergquist, 1994; Tanner, 1996; Bentley et al., 2001; Mercier et al., 2011). Reproduction requires that an organism has available energy for gamete production and thus, nutrient availability has also been positively correlated with gametic/larval density (Thompson, 1983; Snell, 1986). Many organisms reproduce seasonally and rely on cyclic patterns for annual mass spawning events. Seasonality of reproduction is thought to be driven by limited resources, where during periods of low food availability, organisms must instead allocate energy to other processes including maintenance (i.e., respiration, excretion), defence and growth (Olive, 2011). Therefore, phenology is often driven largely by the cyclic flux of food and other associated environmental changes (e.g., temperature, photoperiod).

An annual mass synchronised event by which all offspring are released at once can be evolutionarily advantageous because a high number of gametes can overwhelm potential predators; further, gametes have a higher chance of interacting (i.e., fertilization), thus increasing the likelihood of outbreeding and overall survival of larvae (Guest et al., 2005). Examining how cyclic patterns, such as the El Niño-Southern Oscillation (ENSO), influence seasonally reproducing marine invertebrates can highlight how changes in the surrounding environment can have marked consequences on such mass release events. For example, global and rapid changes of both temperature and rainfall that occur during ENSO events have been recorded to change biological productivity, resulting in cascading effects on survival and reproduction at higher trophic levels (Glynn, 1988; Richmond, 1990). For instance, sea temperature rises were associated with increased spawning across different phyla in Lough Hyne, Ireland, where increased numbers of larvae were positively associated with temperature 
(Minchin, 1992). Furthermore, changes in currents and salinity associated with ENSO events often change spawning grounds and larval transport pathways, leading to shifts in population abundance and structure (Hsiung et al., 2018). For instance, upwelling events and warm temperatures associated with ENSO events have resulted in increased larval transport from deep-sea to inshore areas for both flounder and halibut (Bailey and Picquelle, 2002). Rapid changes in the environment projected with future climate change are therefore likely to alter reproductive timing, duration, frequency and output for organisms; and such changes to reproduction are likely to have consequences for population dynamics if organisms cannot adapt quickly enough (Kroeker et al., 2010; Byrne, 2011). As reproduction ultimately shapes population growth and connectivity, understanding how the environment influences reproduction is key for predicting future viability of populations and making effective management and conservation decisions.

Sponges play important functional roles in tropical, temperate and polar ecosystems (Bell, 2008), and serve as excellent models for understanding how the environment may influence complex reproductive strategies. Despite lacking specialized reproductive organs like gonads, sponges have evolved numerous strategies to reproduce (see Maldonaldo and Riesgo, 2008a for detailed review). Sponges can reproduce sexually by either gonochorism or hermaphroditism and for gametic development, either release eggs (ovipary) or internally brood larvae (vivipary) (Ilan and Loya, 1990; Baldacconi et al., 2007; Riesgo and Maldonado, 2008; Abdul Wahab et al., 2017). In conjunction with sexual reproduction, sponges also reproduce asexually, where genetically identical clones are produced by budding, fragmentation or gemmulation (Simpson and Gilbert, 1973; Johnson, 1978; Corriero et al., 1998; Teixidó et al., 2006). With respect to seasonality, some sponges sexually/asexually reproduce continuously throughout the year, while others may have one short sexual and/or asexual reproductive event per year (Hoppe, 1988; Riesgo and Maldonado, 2008; Lanna et al., 2014; Nozawa et al., 2016). Such variability in reproduction across the phylum allows an excellent opportunity to explore how the environment influences reproduction and how different strategies may respond differently to changing climates. Numerous studies have highlighted the effects of different environmental factors on reproduction in sponges. Reproductive effort has been shown to be highly correlated with nutrient availability, salinity, rainfall, oxygen and sediment/turbidity (Witte, 1996; Roberts et al., 2006; Whalan et al., 2007b; Gaino et al., 2010; Abdul Wahab et al., 2014). Light, lunar and tidal cycles also play a 
role in reproductive timing, often triggering spawning events (Amano, 1986; Amano, 1988; Hoppe and Reichert, 1978; Usher et al., 2004; Nozawa et al., 2016). Among the most studied environmental influences, however, is temperature. Both decreases and increases in temperature have been shown to coincide with gametogenesis, biased sex-ratios and increased sexual reproductive output for a number of sponges (Reiswig, 1973; Usher et al., 2004; Ettinger-Epstein et al., 2007; Riesgo et al., 2007; Whalan et al., 2007a; Riesgo and Maldonado, 2008; Ereskovsky et al., 2013; Abdul Wahab et al., 2014; Lanna et al., 2014; Abdul Wahab et al., 2017). Despite the large a number of studies, however, common patterns of reproduction in relation to environmental parameters for sponges have not emerged, highlighting speciesspecific reproductive adaptations.

New Zealand contains a diverse and rich assemblage of sponges (Bergquist and Warne, 1968; Van Soest et al., 2012), and consequently many gaps exist in our knowledge of the reproduction of sponges existing here. Ayling (1980) examined patterns of both sexual and asexual reproduction for a number of northern New Zealand demosponges, but with little consideration of potential environmental drivers. A few relevant studies have emerged since, but these have only examined reproduction in the context of connectivity patterns (Miller et al., 2001) or chemical ecology (Duckworth and Battershill, 2001), and have lacked histological detail of the basic reproductive ecology of these temperate sponges. Here, I investigated the reproductive ecology of sponges in the genus Tethya in Wellington, New Zealand, with the following objectives: (1) to describe the previously unknown reproductive biology of both $T$. bergquistae and the T. burtoni complex (see Chapter 2) at two different locations in Wellington (Breaker Bay and Princess Bay); (2) to determine if differences in sexual reproduction exist between $T$. bergquistae and the T. burtoni complex and between sites; and (3) to describe potential environmental correlates that may be important in reproduction for these species. Despite being a common genus in the New Zealand marine environment (Sarà and Sarà, 2004; Berman, 2012), there are no detailed studies examining the reproduction of these Tethya species to date. 


\section{Methods}

Sample collection

From February 2015 to 2017, twenty specimens of each species (i.e., ten of Tethya bergquistae and ten from the T. burtoni complex) were collected monthly using SCUBA from two separate locations on the Wellington south coast, New Zealand (Breaker Bay: 41 ${ }^{\circ} 19 ' 58.6056^{\prime \prime} \mathrm{S}$, 174²9'53.1732" E; and Princess Bay: 4120'49.4016" S, 174²7'24.6732" E; see Chapter 5, Figure 5.2 for a map), totalling 40 sponges per month. Sponges were collected from $5-10 \mathrm{~m}$ depth. Tethya bergquistae and the T. burtoni complex are morphologically distinguishable from each other in that the former is pink-orange in colour, firm and has variable surface texture, from smooth to bumpy; whereas the latter is yellow-orange in colour, soft and generally covered in small irregular tubercles (Hooper and Wiedenmayer, 1994; Bergquist and Kelly-Borges, 1991; Sarà and Sarà, 2004). Members belonging to the T. burtoni complex could not be distinguished from one another based on morphology alone (see Chapter 2), and so all specimens are presented here as one group, despite the fact that there is likely cryptic speciation in the group. Sponges were immediately fixed in Bouin's fixative for $48 \mathrm{~h}$ and then preserved in $70 \%$ ethanol. As Tethya species have a spherical morphology, sponge size was estimated as the volume of a sphere after measuring the diameter (in $\mathrm{mm}$ ) for all sponges using calipers.

\section{Histological processing and quantification of reproductive propagules}

An approximately $5 \mathrm{~mm}$ thick slice of tissue from each sponge was processed for histological analysis, where tissue was dehydrated with a series of ethanol solutions (70\% to $100 \%$, increasing $10 \%$ each step), cleared with xylene and infiltrated with paraffin wax over $16 \mathrm{~h}$ using an automated processor (Leica Biosystems TP1020). Tissue was embedded in paraffin wax using an embedded centre (Leica Biosystems EG1160), and sectioned to $5 \mu \mathrm{m}$ using a rotary microtome (Leica Biosystems RM2235). Sections were stained using haematoxylin and eosin staining, and mounted on slides with DPX. Sections were examined at 100-200x magnification for the presence of gametes (oocytes and spermatic cysts) using a compound microscope (Leica Microsystems DM LB) with attached digital camera (Canon EOS 70D). For sponges containing gametes, five separate (i.e., not overlapping) fields of view per section were photographed. The number and size (surface area of oocytes or spermatic cysts), as well as the total tissue area viewed, were calculated using ImageJ (Schneider et al., 2012). The reproductive output index (ROI) was calculated for both species as the proportion of tissue 
containing reproductive entities (i.e., the sum of the areas of reproductive entities divided by the entire tissue area examined, as in Corriero et al., 1998; Whalan et al., 2007a,b). ROI is an accepted measurement for comparing reproduction between different sponges and has been employed in many recent studies (Leong and Pawlik, 2011; Whalan et al., 2007a,b; Abdul Wahab et al., 2014; Abdul Wahab et al., 2017).

\section{Collection of environmental data}

Environmental factors that were considered to potentially influence reproduction in Tethya were sea surface temperature (SST), concentration of chlorophyll- $a$ (chl- $a$ ), and rainfall, which have all previously been found to help explain variation in sponge reproduction (Roberts et al., 2006; Whalan et al., 2007a,b; Abdul Wahab et al., 2014). For instance, reproduction in relation to temperature has been studied extensively for sponges, and no general pattern has emerged but instead both increases and decreases in temperature have been found to trigger gametogenesis (Riesgo and Maldonado, 2008). Chl- $a$ concentration was used here as an indication of food availability. Sponges feed on heterobacteria, picoplankton possessing chl- $a$, and picoplankton possessing chlorophyll types other than chl- $a$ (Reiswig, 1975; PereaBlàzquez, 2011). While chl- $a$ concentration may not reflect all sponge food types, it was used here instead as an estimate to identify seasonal fluxes in nutrients and productivity (Yoder et al., 1993; Murphy et al., 2001). Food availability has been associated to reproduction for many marine invertebrates (Vance, 1973; Bronstein and Loya, 2015), and therefore was considered a potentially important driver of reproduction. Lastly, rainfall has been found to be correlated with reproduction for sponges (Elvin, 1976), corals (Mendes and Woodley, 2002), and other benthic organisms (Paixão et al., 2013). Levels of rainfall may be used to give an indication of cloud cover (photoperiod), changes in salinity or levels of sedimentation.

Sea surface temperature $\left({ }^{\circ} \mathrm{C}\right)$ and chl- $a$ concentration $\left(\mathrm{mg} \cdot \mathrm{m}^{-3}\right)$ were obtained from Satellite Data Services from the National Institute of Water and Atmospheric Research (NIWA) in New Zealand. NIWA Satellite Data Services receives and analyses satellite transmissions from the National Oceanic and Atmospheric Administration (NOAA) and granules from the Moderate Resolution Imaging Spectroradiometer (MODIS) for SST and chl-a concentration, respectively. For each satellite image, data were extracted from a 5 x $5 \mathrm{~m}$ grid around each site (2.5 km square) and the median was calculated. Breaker Bay and Princess Bay are 
approximately $5 \mathrm{~km}$ from each other, and therefore large-scale satellite SST and chl- $a$ concentration readings would be expected to be the same for both sites (which was reflected in the readings obtained for both sites). Data points from satellite images were scarce for Breaker Bay due to the proximity to land, and therefore all values were averaged for the two sites, which generated one dataset to be compared for both locations. Monthly averages of temperature and chl- $a$ were calculated. Total monthly rainfall (mm) was obtained from New Zealand's National Climate Database (https://cliflo.niwa.co.nz/), which offers free real time and archived data from stations across New Zealand. The environmental dataset used here therefore included means of SST and chl- $a$ concentration, as well as total rainfall, for each month.

\section{Data analysis}

All analyses were conducted in R v 3.5.0 (R Core Team, 2017). To determine the effects of potential predictors on instances of sexual reproduction, a generalized linear model (GLM) with a binomial family and logistic link was used. Logistic GLMs allow the probability of a binary response (here, $1=$ sexually reproductive and $0=$ not sexually reproductive) to be estimated from one or more explanatory variables. The logistic link (or logit link) transforms the response (here, sexual reproduction) so that there is a linear relationship to the potential predictors, and the output is the log-odds of the probability of the response as a function of the predictors. The model used here tested the log-odds of the probability of sexual reproduction as a function of: sponge size (diameter), site, species, temperature, chl- $a$ concentration and rainfall. Site (Breaker Bay, Princess Bay) and species (T. bergquistae, T. burtoni) were considered factors. Likelihood ratio (LR) $\chi^{2}$ tests were used to test the significance of the effects of all explanatory variables on the probability of sexually reproducing. A Box-Tidwell procedure with 1,000 iterations was used to check that the model met the assumptions of a logistic regression, which include: (1) linearity of independent variables and log odds; and (2) no multicollinearity of independent variables (Box and Tidwell, 1962). All variables met the second assumption. Temperature, however, failed to meet the first assumption, even after both a natural $\log$ and square root transformation, so another additional model was fitted with temperature as a categorical variable to confirm that the effect of temperature (as a continuous variable) was valid. For this, temperature was placed into four even categorical groups based on clusters of individuals, where $1=<12^{\circ} \mathrm{C}(\mathrm{n}=158$ sponges $), 2=12-14^{\circ} \mathrm{C}(\mathrm{n}=272) ; 3=$ $14-16^{\circ} \mathrm{C}(\mathrm{n}=278)$ and $4=16+{ }^{\circ} \mathrm{C}(\mathrm{n}=270)$. 
To determine the effects of potential predictors on oocyte density, oocyte size and ROI, linear models were used. Because sponges were only sexually reproducing for six of the 25 months sampled during this study period, only those sponges that had gametes were used for these analyses (i.e., all zero values were removed). Oocyte density, oocyte size and ROI were each modelled as a function of: sponge size (diameter), species, site, temperature, chl- $a$ and rainfall. Environmental covariates did not significantly affect oocyte density, oocyte size or ROI (likely due to little variation in environmental variables within each sexual reproduction event), and so these terms were removed from each linear model leaving site, species and size as explanatory variables. Significant effects were assessed using Type-III ANOVAs. All data were checked to meet the assumptions of a linear regression (i.e., normality, homoscedasticity, no multicollinearity or auto-correlation of independent variables; Poole and O'Farrell, 1971), and oocyte density and ROI were log transformed to meet these assumptions. Results remained the same after rerunning the analysis after removing any outliers. No statistical comparisons were made between months as there were too few data points for each month for robust comparisons. A final linear regression was fitted to test the effect of site and species on sponge size, following the same procedures as previously stated. For linear models, three interactions of interest (sitexspecies, species $\times$ size, sizexsite) were tested, but because none were significant, they were not included in the models.

\section{Results}

\section{Environmental data}

Environmental data for the Wellington south coast are summarized in Figure 3.1, and are presented as monthly means for SST and chl- $a$ concentration and as total monthly volume for rainfall. SST followed a seasonal trend, where it peaked in February each year at approximately $17^{\circ} \mathrm{C}$ and then fell to $11^{\circ} \mathrm{C}$ in July/August. Rainfall and chl- $a$ concentration were more variable. Maximum total monthly rainfall occurred in November 2016, which had approximately 250 $\mathrm{mm}$ for the month. Rainfall decreased over the summer period (December - February). Chl- $a$ concentration averaged $1.25 \pm 0.3 \mathrm{mg} / \mathrm{m}^{3}$, and ranged from $0.77-1.93 \mathrm{mg} / \mathrm{m}^{3}$ over the study period. There was no apparent seasonal pattern for chl- $a$ concentration, but there were peaks evident during spring periods. Environmental covariates were not correlated with each other (see Appendix 3, Figure A3 for plots). 

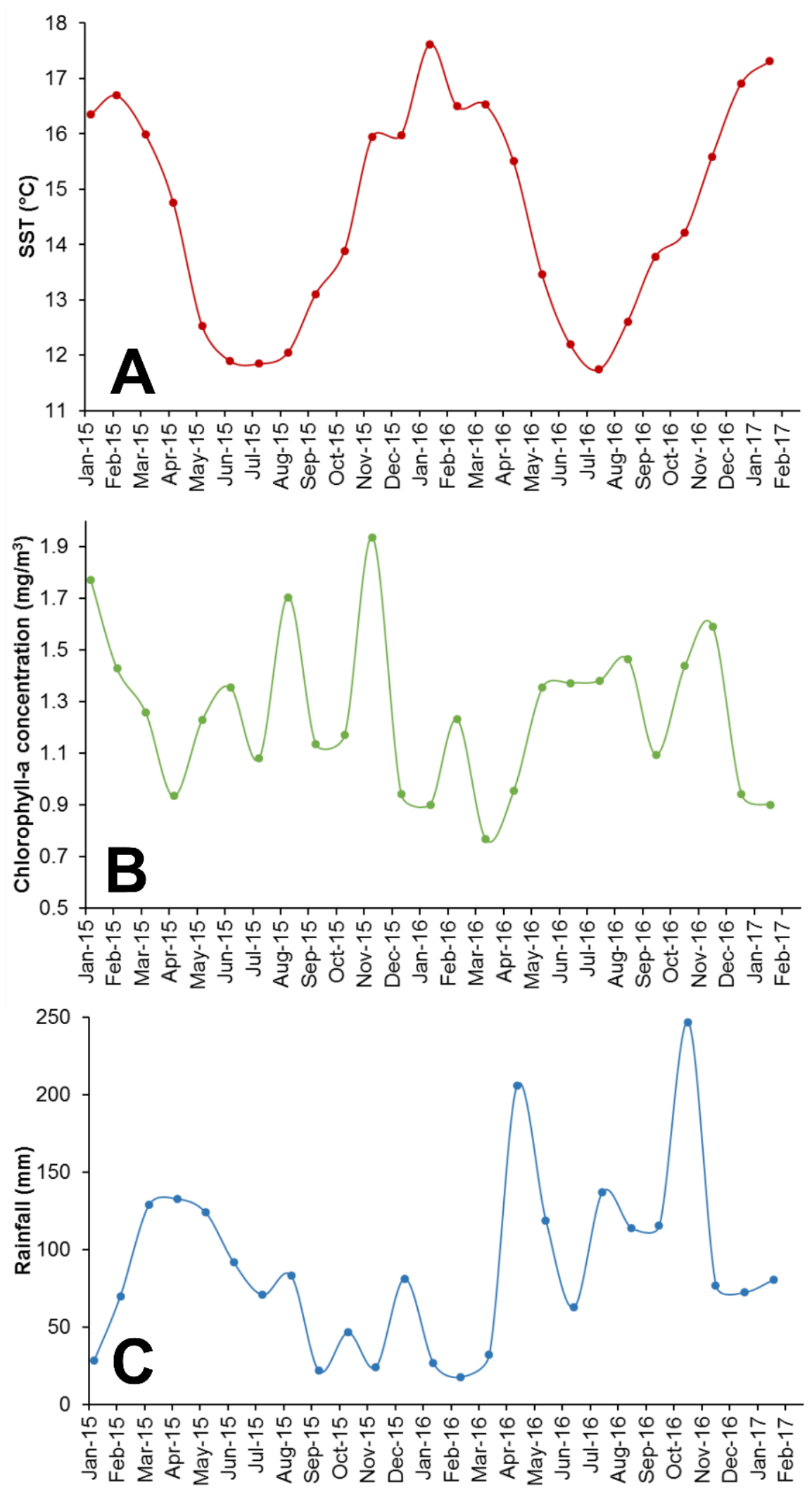

Figure 3.1. Environmental data from the Wellington south coast from January 2015 to February 2017. Monthly means given for sea surface temperature (SST, in ${ }^{\circ} \mathrm{C} ; \mathbf{A}$ ) and chlorophyll- $a$ concentration (chl- $a$, in $\mathrm{mg} \cdot \mathrm{m}^{-3} ; \mathbf{B}$ ), whereas monthly rainfall (in $\mathrm{mm} ; \mathbf{C}$ ) corresponds to total monthly volumes. 
Reproductive ecology of Tethya spp.

Over the two-year study period, 995 sponges were collected, of which 86 contained oocytes and one contained spermatic cysts (see Table 3.1 for summary, Figure 3.2). No sponges were found to contain internally brooded larvae or both male and female gametes, which suggests that $T$. bergquistae and T. burtoni are both oviparous, gonochoristic sexual reproducers. Both species had one sexual reproductive event per year, occurring in the austral summer from January to March (Figure 3.3). One full reproductive event was captured in January - March 2016. For this event, the proportion of T. bergquistae containing gametes decreased from January to March. Tethya burtoni was not found to contain gametes until February 2016, which was a month later than $T$. bergquistae. Reproduction occurred when the temperature reached above $15^{\circ} \mathrm{C}$, and the proportion of sponges containing gametes significantly increased with increasing temperature (LR chi-square test, $\chi^{2}=93.221, P<0.001$; Figure 3.4). The logistic GLM produced a model coefficient (i.e., log odds ratio) of 1.37 for temperature $(P<0.001)$, which allows the prediction that for every $1{ }^{\circ} \mathrm{C}$ increase of temperature, the odds that a sponge will sexually reproduce increases approximately 4 times (i.e., $\mathrm{e}^{1.37}$ ). Reproduction occurred
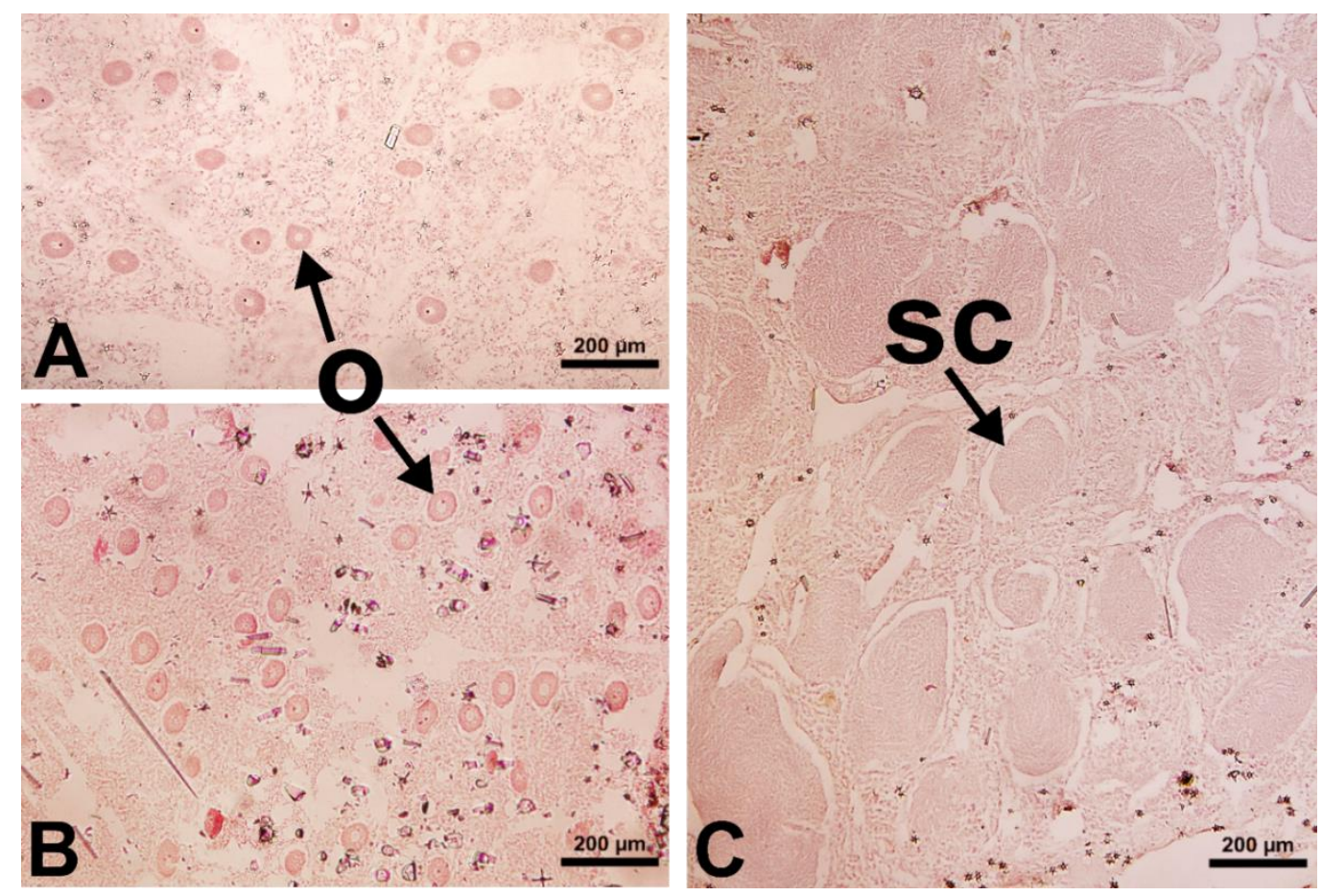

Figure 3.2. Examples of tissue sections containing gametes (oocytes, $\mathrm{o}$; and spermatic cysts, sc). For oocytes, tissues of both Tethya burtoni (A) and T. bergquistae (B) are shown. Spermatic cysts in tissue belonging to T. burtoni (C). 
Table 3.1. Summary information of Tethya spp. collected from Feb 2015-2017. Site refers to Breaker Bay (BB) or Princess Bay (PB). Number of sponges collected (N), number containing oocytes (N-O), and number containing spermatic cysts (N-SC) given for each site, each species, and overall. Mean sponge size refers to the diameter of the sponge $(\mathrm{mm})$. Mean oocyte density (counts per $\mathrm{mm}^{3}$ ) and mean oocyte size (SA of oocyte, $\mu \mathrm{m}^{2}$ ) are averaged across all months. The average reproductive output index (ROI) is given as the $\%$ of tissue sampled with reproductive entities. Standard deviations given for all values.

\begin{tabular}{ccccccccc}
\hline Species & Site & N & $\begin{array}{c}\text { N- } \\
\text { O }\end{array}$ & $\begin{array}{c}\text { N- } \\
\text { SC }\end{array}$ & $\begin{array}{c}\text { Mean } \\
\text { sponge size }\end{array}$ & $\begin{array}{c}\text { Mean } \\
\text { oocyte } \\
\text { density }\end{array}$ & Mean ROI & $\begin{array}{c}\text { Mean } \\
\text { oocyte size }\end{array}$ \\
\hline Tethya & BB & 251 & 23 & 0 & $21.3 \pm 4.5$ & $20.2 \pm 18.4$ & $1.7 \% \pm 1.7 \%$ & $0.82 \pm 0.29$ \\
bergquistae & PB & 250 & 22 & 0 & $19.0 \pm 4.1$ & $12.7 \pm 9.7$ & $1.0 \% \pm 0.8 \%$ & $0.73 \pm 0.27$ \\
& Total & 501 & 45 & 0 & $20.2 \pm 4.4$ & $16.5 \pm 15.1$ & $1.3 \% \pm 1.4 \%$ & $0.78 \pm 0.28$ \\
\hline Tethya & BB & 244 & 23 & 1 & $19.6 \pm 5.9$ & $32.6 \pm 16.4$ & $2.4 \% \pm 1.5 \%$ & $0.72 \pm 0.17$ \\
burtoni & PB & 250 & 18 & 0 & $17.5 \pm 4.5$ & $23.0 \pm 18.4$ & $1.8 \% \pm 1.6 \%$ & $0.72 \pm 0.24$ \\
complex & Total & 494 & 41 & 1 & $18.5 \pm 5.3$ & $28.4 \pm 17.8$ & $2.1 \% \pm 1.6 \%$ & $0.72 \pm 0.20$ \\
\hline Overall & & 995 & 86 & 1 & $19.3 \pm 5.0$ & $22.2 \pm 17.4$ & $1.7 \% \pm 1.5 \%$ & $0.75 \pm 0.25$ \\
\hline
\end{tabular}
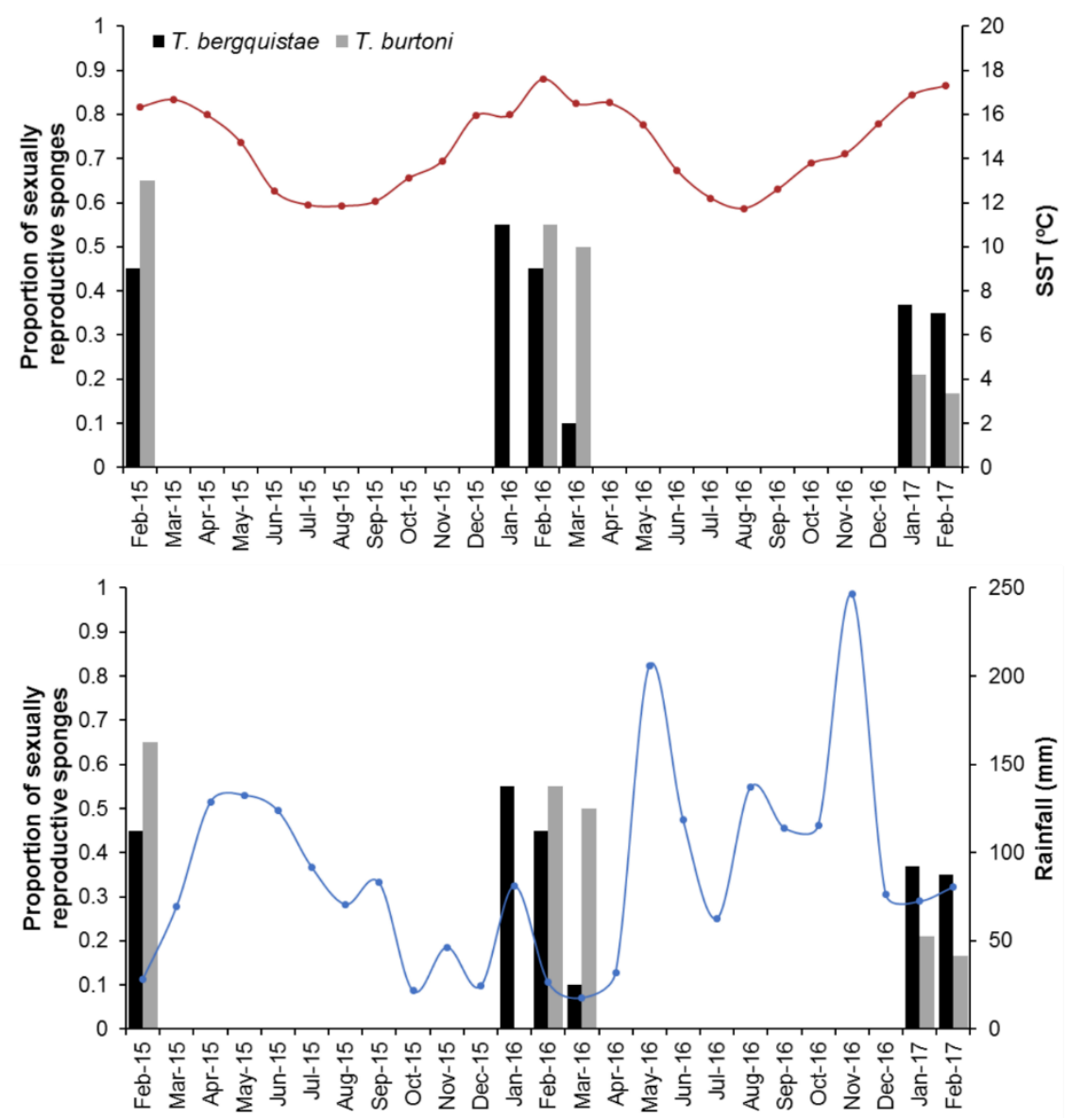

Figure 3.3. Monthly proportion of sexually reproductive sponges from Feb 2015-2017 for Tethya bergquistae (black) and T. burtoni (grey). Monthly means for sea surface temperature (SST, ${ }^{\circ} \mathrm{C}$ ) overlaid on top graph and total monthly rainfall $(\mathrm{mm})$ on bottom. 
during periods of lower rainfall, and total monthly rainfall was negatively correlated to instances of reproduction $\left(\chi^{2}=7.568, P=0.006\right.$; Figure 3.4). The model coefficient for rain was $-0.012(P=0.008)$, which predicts that for every $1 \mathrm{~mm}$ decrease of rainfall, the odds of sexually reproducing is increased by 1.01. There was no effect of chlorophyll- $a$ on the proportion of sponges containing gametes (Figure 3.4).
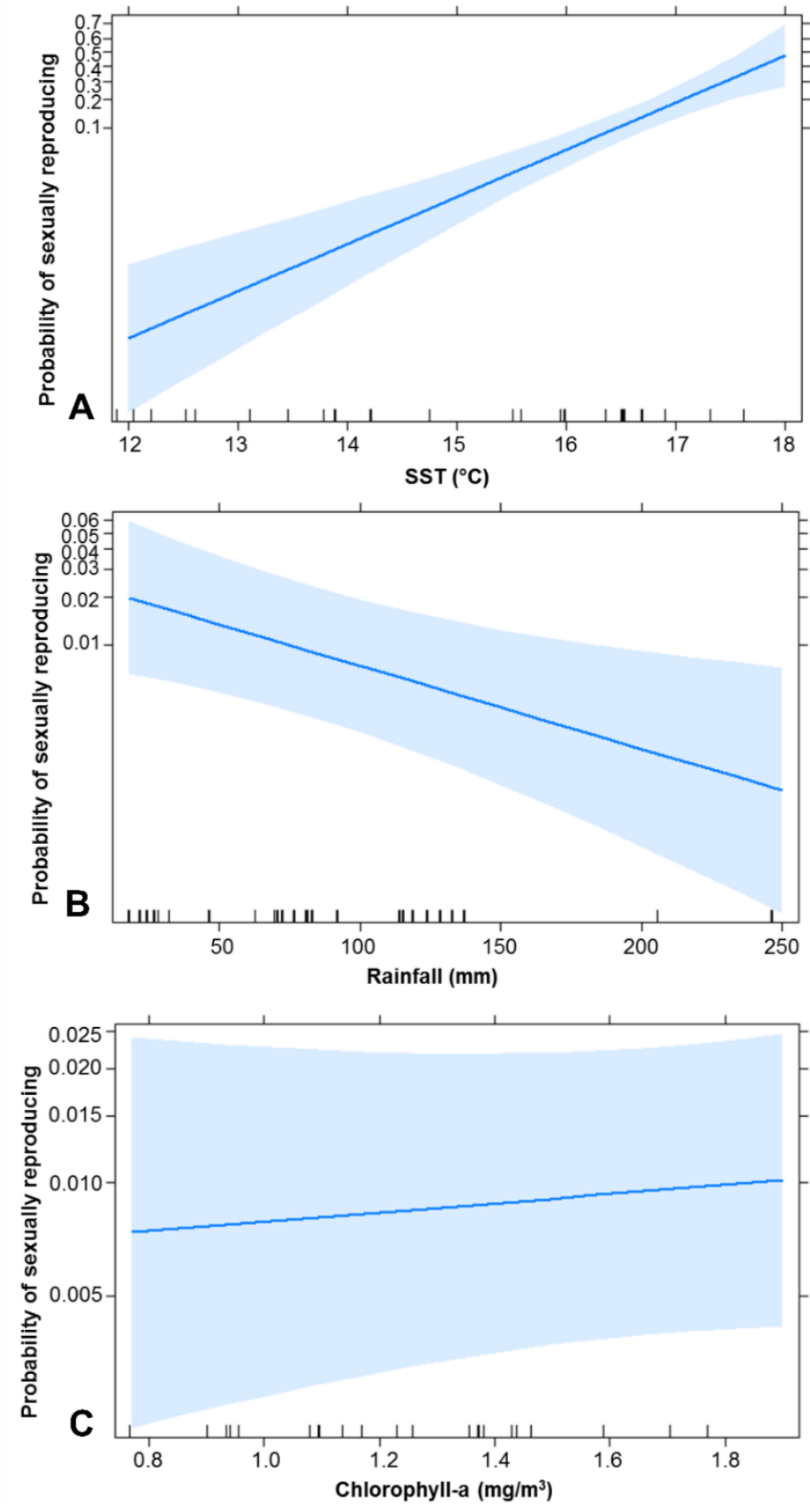

Figure 3.4. Environmental effects on the probability of sexual reproduction by Tethya spp. Environmental effects include: sea surface temperature (A), rainfall (B) and chlorophyll- $a$ concentration (C). Bottom ticks across the $\mathrm{x}$-axes represent an observed response from a sponge, and shaded area around lines represent $95 \%$ confidence intervals. 
In general, oocyte density increased during the reproductive period (Figure 3.5). Oocyte density was significantly different between sites (ANOVA, $\mathrm{F}_{1,82}=10.593, P=0.002$ ) and between species (ANOVA, $\mathrm{F}_{1,82}=13.073, P=0.001$ ). Gamete density was greater at Breaker Bay for all months except two (January and February 2016), and was greater for T. burtoni except for all months except two (February 2015 and January 2016; Figure 3.5). ROI followed the same trend as oocyte density (see Appendix 4, Figure A4 for plot), and was also significantly different between sites (ANOVA, $\mathrm{F}_{1,82}=8.7105, P=0.004$ ) and between species (ANOVA, $\left.F_{1,82}=7.7786, P=0.007\right)$. Gamete size increased over each reproductive event for both species, and was not significantly different between sites or species (Figure 3.6). Tethya bergquistae
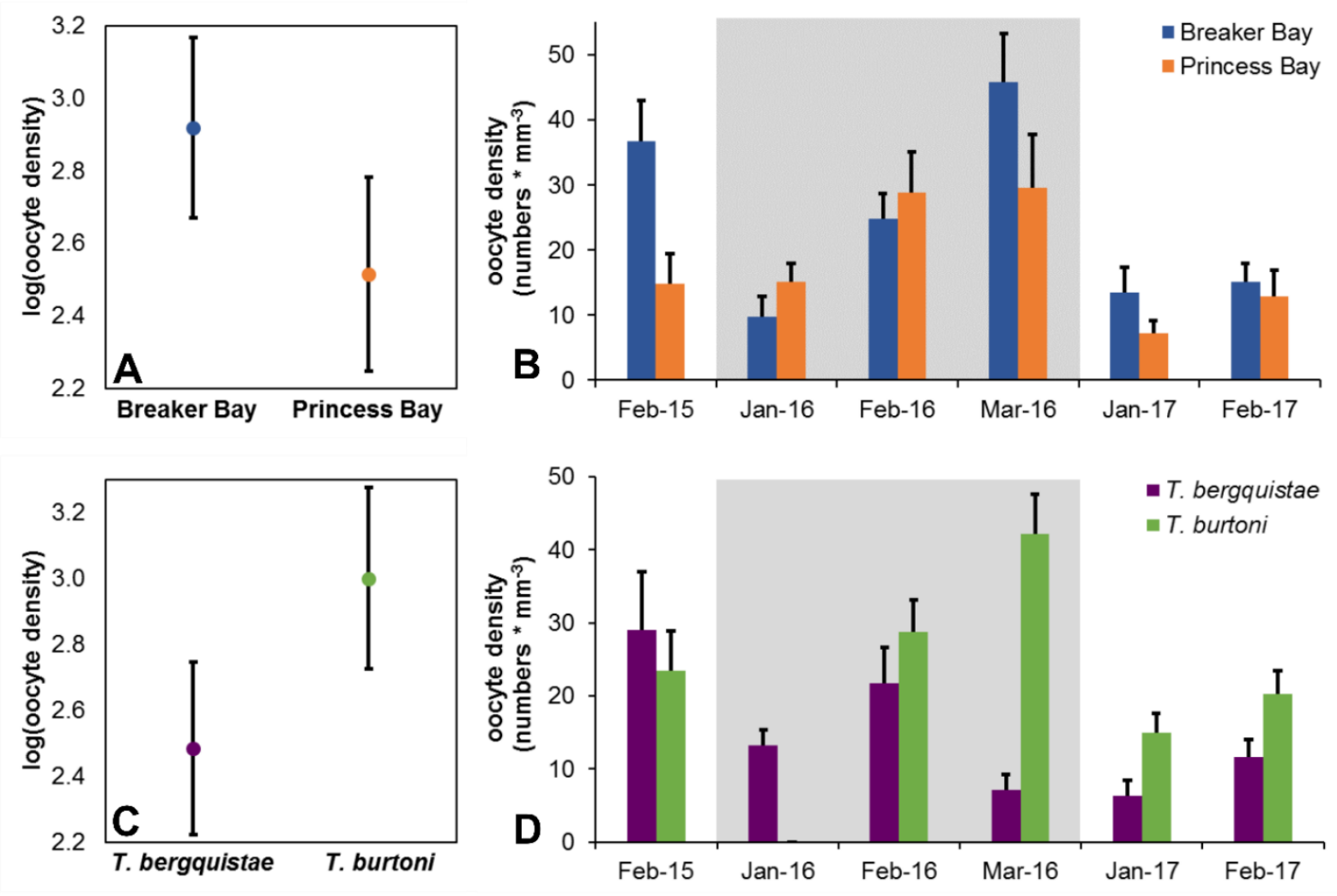

Figure 3.5. The effects of site (top) and species (bottom) on oocyte density for Tethya bergquistae (purple) and T. burtoni (green) from both Breaker Bay (blue) and Princess Bay (orange). Plots on left are means of the $\log$ (oocyte density) between site (A) and species (C), and bars are 95\% confidence intervals. Plots of right are differences in oocyte density between sites (B) and species (D) presented by month, where the entire reproductive event captured is highlighted in grey. Sample sizes for species collected from each site are presented in Table 3.1. Error bars on plots on right correspond to standard error. 
was significantly larger in volume than $T$. burtoni (ANOVA, $\mathrm{F}_{1,991}=30.0784, P<0.001$ ). Furthermore, sponges at Breaker Bay were significantly larger than those at Princess Bay (ANOVA, $\mathrm{F}_{1,991}=52.4956, P<0.001$; Figure 3.7). Size was significantly correlated to the presence of gametes (LR chi-square test, $\chi^{2}=12.416, P<0.001$ ) and the probability of containing gametes increased with size (Figure 3.8A). Size was also significantly correlated with oocyte density and ROI, but not oocyte size (Figure 3.8B-D). Summaries of statistical tests can be found in Appendix 5, Tables A5a, b.

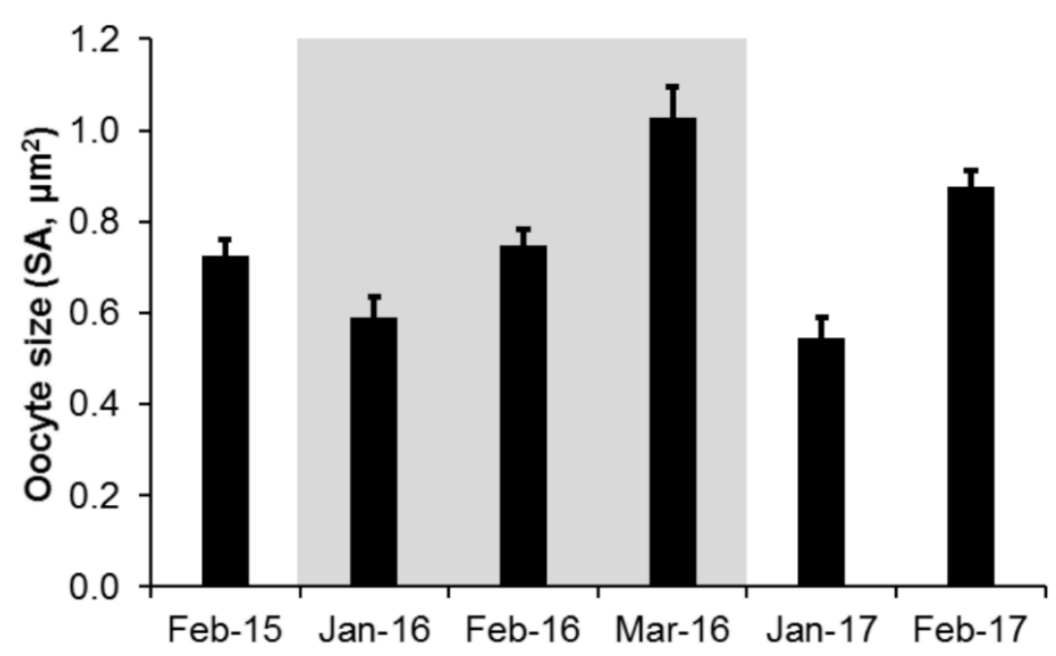

Figure 3.6. Monthly average oocyte sizes (surface area, SA; in square microns) for both Tethya bergquistae and T. burtoni across all sites. The entire reproductive event captured is highlighted in grey, and error bars on plots on right correspond to standard error.

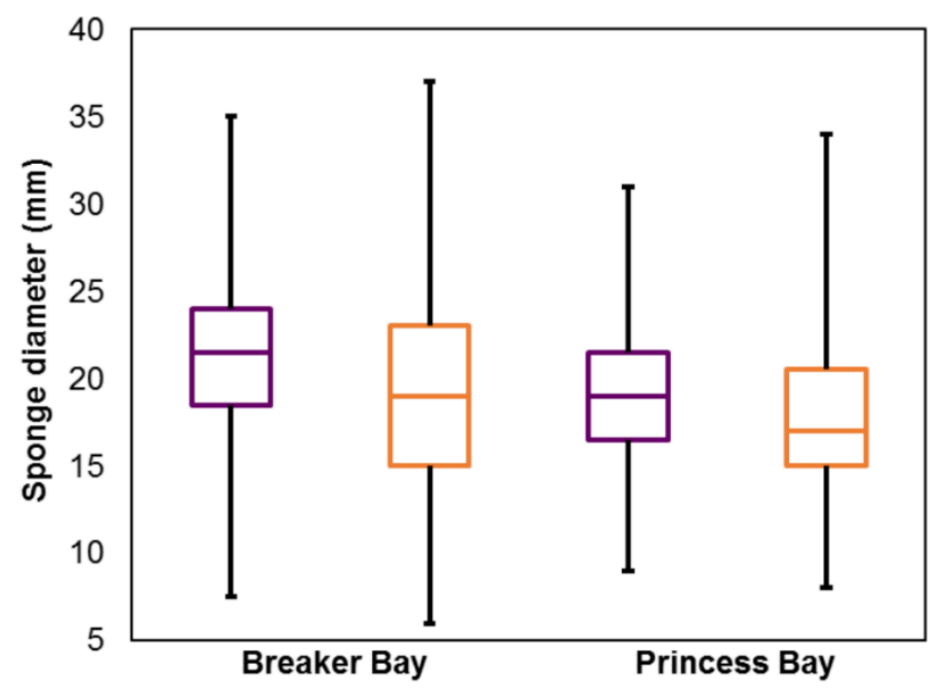

Figure 3.7. Box plot representing differences in sponge size (diameter, $\mathrm{mm}$ ) between Tethya bergquistae (purple) and T. burtoni (orange) from two sites. 

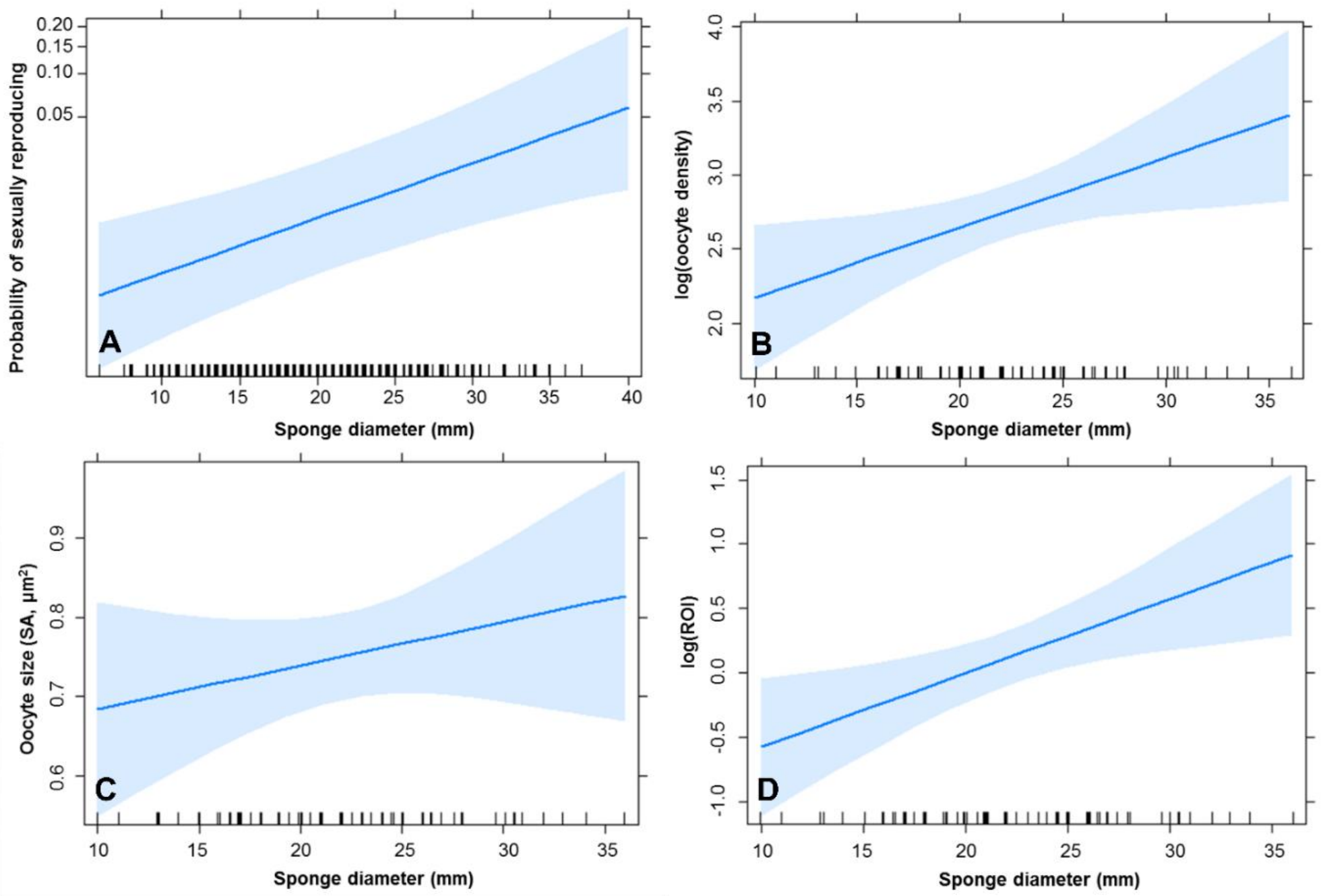

Figure 3.8. The effect of sponge size (diameter, in $\mathrm{mm}$ ) on the probability of sexually reproducing (A), oocyte density (B), oocyte size (C) and ROI (D) for Tethya bergquistae and T. burtoni. ROI, or the reproductive output index, is the percent of tissue occupied by gametes. Bottom ticks across the $\mathrm{x}$-axes represent an observed response from a sponge, and shaded area around lines represent $95 \%$ confidence intervals. 


\section{Discussion}

In this chapter, I described the reproductive ecology of Tethya spp. and investigated potential environmental drivers of sexual reproduction, to assess how reproduction may be influenced by climate change. These sponges were found to have one annual sexual event, with gametogenesis beginning in January and the release of eggs occurring two to three months after gametogenesis. Tethya spp. were found to be gonochoristic, though sequential hermaphroditism cannot be ruled out. The lack of sampled males suggests that spermatogenesis is rapid, and it is likely that my sampling regime was not frequent enough to characterize spermatogenesis. More frequent sampling of the same individual over a sexual reproductive event would be able to address the possibility of sequential hermaphroditism, but all other species of Tethya to date have been recorded to be gonochoristic (Gaino et al., 1987; Gaino and Sarà, 1994; Corriero et al., 1996) so this is also likely the case for T. bergquistae and $T$. burtoni. Despite having the same reproductive period, some characteristics of sexual reproduction in sympatric species of Tethya were found to be different, which may be an indication of reproductive isolation and further support current delineations of both species. Sexual reproduction was correlated with temperature and rainfall, and occurred following periods of higher chl- $a$ concentration, highlighting that seasonal environmental cues may be important for sexual reproduction for Tethya. Understanding how these environmental drivers influence reproduction in Tethya is imperative for predicting how climate change may affect their reproductive phenology as well as population growth and connectivity.

\section{Environmental influences on reproduction}

Reproduction in temperate sponges is often seasonal, where reproductive seasonality is associated with periodicity of environmental variables including temperature, photoperiod, rainfall and nutrients (Ayling, 1980; Usher et al., 2004; Whalan et al., 2007b; Abdo et al. 2008). Such cyclic patterns result in periods of limited resources, resulting in a lack of available energy for organisms to continuously reproduce throughout the year (e.g., Pond et al., 1996). As a result, organisms take advantage of when productivity is high and either store energy for later use or immediately use available resources, as both strategies allow organisms to reproduce when conditions are favourable. The time of year found to be conducive for reproduction in Tethya spp. occurred during the austral summer, which is characterized by warmer waters, lower rainfall and decreasing available nutrients. Chlorophyll- $a$, which was used here as a 
proxy for productivity, was not correlated with sexual reproduction in $T$. bergquistae or $T$. burtoni. High levels of chl- $a$ were recorded in the spring months (September to November, see Figure 3.1), which is similar to previous findings describing the Wellington south coast. For instance, the Wellington south coast undergoes seasonal fluctuations in nutrient availability, where chl- $a$ blooms in the autumn and spring periods (Murphy et al., 2001). Winter periods in the New Zealand waters are characterized by higher nutrients, whereby productivity is light limited; summer periods, by contrast, are typified by secondary productivity and the associated depletion of nitrates (Bradford and Roberts, 1978; Murphy et al., 2001; Perea-Blàzquez, 2011; Tam, 2012). There is a lag of time from the onset of primary productivity in the winter/spring to when secondary production occurs, in which the biomass of picoplankton and other heterotrophic bacteria increase (Pomeroy, 1991; Tam, 2012). The diet of Tethya has been found to consist of both heterotrophic bacteria and cyanobacteria (Prochlorococcus and Synechoccus, Perea-Blàzquez, 2011), and it is likely that during the winter and spring periods, Tethya accumulate energy with increasing food sources, and then begin reproduction when nutrients begin to decrease and waters warm. Such a lag in nutrient supply from primary producers to higher trophic levels has been recorded (De Almeida Fernandes et al., 2017), and could also explain why reproduction in these sponges occurs during periods of depleted nutrients. Further, capital breeding, or the process by which organisms store energy and utilize it later, has been recorded for many marine invertebrates (Varpe et al., 2009; Kuklinski et al., 2013; McBride et al., 2013). Such a strategy may be employed by Tethya spp. to alternatively explain the lag in reproduction from nutrient availability. Therefore, while no correlation between chl- $a$ concentration and reproduction was observed, it is still possible that food availability in these temperate waters play a key role in the seasonality of reproduction. Experimental manipulation of nutrient availability and reproductive ability may shed better light on the exact reliance of reproduction on food sources, as in Roberts et al. (2006).

Temperature has also been associated with reproduction for many other sponges, where the production of gametes coincides with periods of both temperature increases and decreases (Fell, 1976; Ettinger-Epstein et al., 2007; Riesgo and Maldonado, 2008; Lanna et al., 2014). For $T$. bergquistae and T. burtoni, gametogenesis began as the temperature increased above approximately $15^{\circ} \mathrm{C}$ in January. Gametes then increased in size and density as the temperature increased to $17^{\circ} \mathrm{C}$ from January to March, and were released when the temperature began to drop in March. Temperature was shown to have a significant effect on the probability of a 
sponge containing gametes, and the GLM model predicted that for every $1{ }^{\circ} \mathrm{C}$ increase, the odds of a sponge sexually reproducing increased by four times. It is important to note, however, that while there was a positive association, there are likely upper temperature limits, with temperatures of $>17-18^{\circ} \mathrm{C}$ being detrimental, as these sponges are not adapted to warmer water (see experiment in Chapter 4). For instance, increased respiration rates as well as increased mortalities have been recorded for T. bergquistae at temperatures above $19^{\circ} \mathrm{C}$ (Bates, 2015), indicating that reproduction may be compromised under thermal stress. Instead, it is likely the seasonality of temperature that is important for reproductive phenology in Tethya. Rainfall has also been previously shown to be associated with sponge reproduction (e.g., Elvin, 1976; Abdul Wahab et al., 2014). While correlations to rainfall may be a product of seasonality, examining reproduction in relation to increased rainfall may also give an indication to how organisms function under reduced salinity and sunlight (cloud cover), as well as under increased sedimentation due to terrestrial runoff. Instances of sexual reproduction in both Tethya spp. was negatively associated with rainfall, but there was no effect of rainfall on oocyte density, ROI or oocyte size. Therefore, predictions of reproductive output based on changes in salinity/sedimentation due to rainfall are difficult. It is instead more likely that this association to rainfall is a product of its seasonality, as during the austral summer rainfall decreases and photoperiod increases in Wellington (https://cliflo.niwa.co.nz/; Tait and Turner, 2005). Other drivers including temperature, photoperiod and productivity may be better predictors, which is reflected in the larger log-odds ratio of temperature compared to rainfall. Nonetheless, sexual reproduction and mass spawning events for some marine organisms that reproduce seasonally have been recorded to occur during periods of calmer weather (Van Woeski, 2010). Wellington summers generally see a decrease in both rain and occurrences of strong southerly storms with high wave energy (Carter, 2008). Spawning during this period would likely increase the chance of fertilization as calm waters would reduce stress on parent sponges and allow available energy for reproduction; furthermore, calm waters would also allow gametes to be retained, increasing their overall chance of survival. As such, it may be evolutionarily advantageous for Tethya spp. to reproduce during this time.

For organisms that rely on environmental cues for life processes, predicted shifts in climate are likely to have serious consequences. Over the past century, warming trends in New Zealand have revealed a complex history (Shears and Bowen, 2017), making it difficult to predict exactly what the environment may look in the next century. It is generally believed that global 
warming will result in a tropicalization of temperate systems (Vergés et al., 2014), but recent findings by Shears and Bowen (2017) are challenging this notion. These authors found that over the past fifty years, changes in the circulation of the South Pacific subtropical gyre have led to warming in southern New Zealand, but little to no warming in central and northern New Zealand. Instead of a tropicalization of the New Zealand marine environment, these authors argue that instead there will be a homogenization of the present day gradient of environments in New Zealand (subtropical to subpolar). Projections of climate change in New Zealand oceans were recently published by Law et al. (2017), where these authors used Earth Model Systems and different IPCC scenarios to predict the largest changes to be observed by 2100 . Using these models, it was predicted that temperatures are to increase by $0.8-1^{\circ} \mathrm{C}$ in the next mid-century and by $1.1-2.5^{\circ} \mathrm{C}$ by the end of the century, with southern New Zealand experiencing the highest degree of warming. On the whole, the following were predictions made for New Zealand oceans: SST will increase by $+2.5^{\circ} \mathrm{C}$; primary production will decrease by $4.5 \%$; and macronutrient concentrations will decrease by $8-20 \%$. Because southern New Zealand is suggested to experience the most change, Tethya spp. in central New Zealand may be better placed than those sponges found more south. Further, if an homogenisation occurs, as indicated by Shears and Bowen (2017), then the diversity of Tethya spp. may decrease.

Changes in phenology, or the timing of seasonal processes in an organism, have already been recorded for many taxa and have been attributed to climate change (Walther et al., 2002). For many marine invertebrates, reproductive cycles can be linked to temperature, photoperiod and other cyclic environmental cues, and there is evidence that changes in such cues alter gamete production, the timing of spawning and sex determination (Lawrence and Soame, 2004). Experiments manipulating temperature have revealed that organisms have a specific thermal range for normal life-history processes (i.e., growth, development, reproduction), and that temperatures exceeding normal ranges may have detrimental effects on such processes (Lee et al., 2003; Byrne et al., 2009). Despite great interannual variability in environmental factors observed throughout this study (see Figure 3.1), recognizable shifts in seasons have been recorded over the last fifty years in New Zealand, where there is a delay in cooling/the onset of winter (Shears and Bowen, 2017). Furthermore, projected changes to nutrient concentrations and productivity as indicated by Law et al. (2017) can potentially lead to mismatches in phenology and nutrient availability, which has consequences for population dynamics. Mismatch for some species has already been documented, where the disparity between the need 
and availability of resources has resulted in organisms failing to obtain enough energy for basic processes like reproduction (Durant et al., 2007). Food limitation is also likely to restrict growth in these Tethya species, which may result in declines in fecundity, given that larger sponges have a significantly higher oocyte density and ROI. While it is unclear how Tethya will respond to changing environmental conditions, their reliance on environmental cues for seasonal reproduction suggests that they are at risk of phenologyproductivity mismatch and reproductive declines as our oceans change, if they cannot adapt quickly enough. More research in relation to temperature, food availability and reproductive timing/output would be able to allow for better prediction for how these species may cope.

\section{Site- and species-specific responses}

Differences in oocyte density and ROI between sites were observed, where Breaker Bay contained sponges that had a higher oocyte density and a higher ROI than Princess Bay. Breaker Bay is at the entrance to Wellington Harbour and, compared to Princess Bay, is more sheltered. The high wave energy experienced at Princess Bay may result in increased stress, leaving less available energy for reproduction by Princess Bay sponges. For instance, both Wilkinson and Evans (1989) and Duckworth et al. (2004) found limited growth of shallow water sponges $(5-10 \mathrm{~m})$ occurring at high levels of turbulence, attributing this to reduced feeding and/or the investment of resources to damage repair and/or stronger skeletal structures. Breaker Bay is also different from Princess Bay with respect to its sediment load, as Breaker Bay experiences inputs of sediment from the harbour. Sedimentation has been shown to negatively affect reproduction for many sponges due to the fact that sponges must allocate energy to stress associated with sedimentation (i.e., sediment clearing, increased respiration) instead of to reproduction (Roberts et al., 2006; Whalan et al., 2007b). However, sedimentation may also result in no consequences to sponge physiology or may actually be beneficial (see Bell et al. 2015 for review). Sediment fluxes from the harbour may bring extra nutrients from the harbour, while southerly storms/tide changes act to remove sediment so that sponges are not subject to prolonged negative effects of deposited sedimentation. While sitespecific environmental measurements would provide more clarity on environmental differences between sites, the results here highlight that reproduction is site- and thus environmentally-sensitive. Sponge size was also significantly different between Breaker Bay and Princess Bay, with sponges being larger at Breaker Bay. This further suggests that Princess 
Bay sponges are perhaps experiencing another form of stress that is resulting in less resources for growth and reproduction. Alternatively, sponges at Princess Bay may be smaller because they are younger and large adults do not survive. However, size/age relationships for Tethya are currently unknown, making it difficult to hypothesize further. Differences in reproduction across proximal sites have also been observed for other sponges. For instance, Whalan et al. (2007b) found that over a range of $80 \mathrm{~km}$, sponges offshore had an ROI 15x higher than inshore sponges, attributing differences to a sediment/light gradient decreasing from the shore. It is possible that water movement and sediment differences between sites in the current study drive the differences observed here.

Both $T$. bergquistae and $T$. burtoni live in sympatry and occupy the same habitat, making barriers to reproduction not obvious. Delineation of species is often made on the basis of reproductive isolation (Mayr, 1942), and here, differences in reproduction were evident between both species. For instance, $T$. burtoni contained a higher density of gametes and a higher ROI compared to T. bergquistae. Because oocyte size did not differ between species, such differences in densities were not a product of differences in oocyte size, but instead $T$. burtoni had a significantly higher reproductive output than $T$. bergquistae. While significant differences in oocyte density and ROI may indicate reproductive isolation, the timing of reproduction for both species was the same. There was a lag in reproduction for T. burtoni over the 2016 sexual reproductive event, but there still remained an overlap in reproductive timing for both species. Furthermore, the other partial reproductive events captured in 2015 and 2017 provide no evidence for asynchrony between the reproductive timing of both species. Evidence for possible introgressive hybridization between $T$. bergquistae and $T$. burtoni has been previously found (see Chapters 2 and 6), and the simultaneous timing of reproduction in both species further supports this hypothesis by indicating a possible mechanism by which this occurs. For instance, gametes from both species are in the water column at the same time and could therefore come into contact to form hybrids. Furthermore, hybridization has been shown to occur by fusion of larvae in both sponges (Maldonado, 1998; McGhee, 2006) and corals (Hatta et al., 1999), and it a strong possibility that fertilized eggs/larvae of both $T$. bergquistae and T. burtoni fuse to form hybrids. Thus, the observed shared reproductive timing of both sponges provides further evidence to support the notion that the boundary between species may be semipermeable, which has also been recorded in other sessile marine invertebrates (Ladner and Palumbi, 2012; Harrison and Larson, 2014; Riesgo et al., 2016). 
While sponges within the T. burtoni complex could not be classified into their various cryptic species (see Chapter 2) because genotyping was not conducted, reproductive timing was nonetheless the same for all Tethya, indicating that differential timing of reproductive events is not a barrier to reproduction between these species.

\section{Conclusion}

In this chapter, I characterize the previously unknown sexual reproductive behaviour of both Tethya bergquistae and T. burtoni on the Wellington south coast. These Tethya species were found to be gonochoristic, oviparous and seasonal sexual reproducers. These results highlight that seasonal variations in both temperature and rainfall are important environmental drivers of reproduction for these sponges. Because these sponges rely on seasonal environmental cues, future shifts in climate that alter seasonal patterns will likely have detrimental effects on reproduction if these sponges cannot adapt to the changes. Such changes in reproduction have the ability to alter population density, connectivity and overall population dynamics, which could lead to longer-term negative consequences for population viability. Furthermore, these results also highlight that reproductive responses for Tethya spp. are both site- and speciesspecific. Site- and species-specific responses should become important considerations during management decisions, as implementing broader-scale plans has the potential to result in differential population responses. 



\section{CHAPTER 4:}

POTENTIAL ENVIRONMENTAL DRIVERS OF ASEXUAL REPRODUCTION IN TETHYA SPP. 



\section{Introduction}

Asexual reproduction, or the mechanism by which organisms produce genetically identical copies of themselves, is commonly employed by many marine taxa. The surrounding environment generally drives the evolution of reproductive strategies, and an array of different environmental conditions have been found to favour asexual reproduction. For example, instances of asexual reproduction in some organisms are high in disturbed areas, where asexual reproduction allows exponential population growth and quick recolonization of an area (e.g., Karlson, 1986; Rasheed, 2004). For other organisms, asexual reproduction is more prominent under stable environmental conditions, where increased genetic variation associated with sexual reproduction is less necessary for population adaptability and survival (Ayre, 1984; Vrijenhoek, 1989). The influence of nutrient and food levels has also resulted in inconsistent responses of asexual reproduction. For instance, high levels of food have been positively associated with asexual budding events for hydra (Tökölti et al., 2016). However, often during periods of increased food availability, organisms display lower instances of asexual reproduction because increased resources results in energy to support sexual processes (Sebens, 1980; Kettenring et al., 2011). Asexual reproduction, instead, is generally more energetically inexpensive and thus more efficient (e.g., Sebens, 1979), and therefore may be employed when food levels are lower and organisms do not have enough energy stores for sexual reproduction. The association of asexual reproduction with many other environmental parameters has been recorded for many marine organisms (including rotifers, cnidarians, corals, sponges and bryozoans), and important environmental correlates include: temperature, salinity, habitat availability, water motion and nutrients (Lubzens et al., 1985; Snell, 1986; O’Dea and Okamura, 1999; Baums et al., 2006; Zilberberg et al., 2006; Purcell, 2007).

Understanding potential drivers of asexual reproduction is important for predicting population growth and overall dynamics. For instance, Purcell et al. (1999) and Purcell (2007) examined clonal production for cnidarians in the Chesapeake Bay (Maryland, USA) to better explain factors leading to jellyfish outbreaks, finding that nutrients, temperature, salinity and solar radiation all influence asexual reproduction. These results had important implications because jellyfish outbreaks have been shown to negatively affect tourism and fisheries, resulting in both ecological and economic consequences. Knowledge of asexual processes can also be critical for managing invasive species because asexual reproduction is often a prominent way in which 
such species successfully colonize a new area and expand their range (Prentis et al., 2008; Silvertown, 2008; Capel et al., 2017). Knowing conditions that are favourable or unfavourable for clonal reproduction for invasive species, therefore, may help allow management to reduce impacts of invasions and thus preserve native diversity. Understanding potential drivers of asexual reproduction may also allow predictions to be made about population dynamics under future changing environments resultant of eutrophication and thermal stress. For instance, increases in asexual reproduction may have been linked to eutrophication and decreased dissolved oxygen concentrations for Aurelia aurita (Ishii et al., 2008), which were proposed to contribute to the observed increased population abundance of this organism. Changes in the frequency and output of asexual reproduction have consequences on the genetic structure of populations, often resulting in a reduction of diversity. Because genetic diversity is associated with population adaptability and longevity, increases in asexual reproduction may lead to inbreeding and population collapses (Muirhead and Lande, 1997; Williams, 2001).

Sponges are found in both marine and freshwater ecosystems in tropical, temperate and polar climates, and serve many important ecological roles from water filtration, nutrient cycling and habitat creation (for a review of functional roles, see Bell, 2008). All sponges reproduce both sexually and asexually (Simpson, 1980). Asexual reproduction can occur in three main ways: budding, fragmentation and gemmulation (Maldonado and Riesgo, 2008). Budding occurs when small spherical bodies project from stalks on the parental sponge, detach and then settle to the substrate (Gaino et al., 2006; Simpson, 2012). Buds generally have spicules to allow attachment to the substrate, and are composed of cells that can give rise to any cell type in the sponge (called archeocytes), which allow the buds to forgo metamorphosis that is required in larvae (Hammel et al., 2009; Cardone et al., 2010). Buds grow into sponges that are clones of the parent sponge and such clones have been found to produce their own buds in as little time as nine months (Cardone et al., 2010). Asexual reproduction by fragmentation occurs when a sponge is divided into two or more new individual sponges, all comprised of the same genotype (Maldonado and Uriz 1999). Fragmentation generally occurs as a result of disturbance or damage (Wulff, 1994; Rodrigues et al., 2001), but the regenerative abilities of sponges (Duckworth, 2003; Henry and Hart, 2005) allow fragments to quickly recover and survive. Lastly, gemmulation is the process by which sponges produce gemmules, which are masses of archeocytes with extra food stores encased by a protective coating (Rasmont, 1962). Gemmulation is generally associated with extreme environments prone to heavy disturbance 
or drought, where the protective envelope around the gemmule allows dormancy until conditions are favourable for the gemmule to hatch and the sponge to grow. Gemmulation generally occurs in freshwater sponges (e.g., Simpson and Gilbert, 1973), though instances of marine gemmulation have also been recorded (e.g., Fell 1974).

Despite the common occurrence of asexual reproduction throughout Porifera, information on environmental drivers of asexual events for sponges is scarce. Both seasonal changes (Johnson, 1978; Corriero et al., 1996; Corriero et al., 1998) and experimentally increased temperature (Cardone et al., 2008) have been shown to induce budding events. Continuous instances of budding have also been associated with heterogeneous environmental conditions for some sponges (e.g., Cardone et al., 2010), indicating that asexual reproduction may play an important role in recolonization and population maintenance. Teixido et al. (2006), however, found that disturbances associated with iceberg scouring did not correlate to instances of budding in Antarctic hexactinellid sponges. Instead, for other sponges, clonality has been found to be higher in more stable environments (Zilberberg et al., 2006). Such conflicting results suggest that asexual reproduction may not play the same role in population dynamics for different sponge populations. Further, it is difficult to elucidate how mixed reproductive strategies (i.e., asexual reproduction in combination with sexual reproduction) shape populations. MercadoMolina et al. (2011) sought to clarify the contribution of both sexual and asexual reproduction on sponge population dynamics through the simulation of various population models. These authors found that population growth decreased markedly when sexual reproduction was absent, but remained unchanged in the absence of asexual reproduction, indicating asexual reproduction alone may not result in an increase in abundance. Findings like these make it difficult to discern the function of asexual reproduction in sponges. Information regarding the timing of asexual reproduction and potential environmental correlations, however, may shed light on its role in shaping populations and allow a more complete understanding of its continued selection for sponge populations. This is particularly important when considering population viability under future environmental changes, as sessile marine invertebrates that rely heavily on asexual reproduction (like sponges) and cannot actively migrate to favourable environmental conditions have already been identified as being at risk of population declines (Przeslawski et al., 2008). 
The New Zealand marine environment reveals a complex history for warming trends over the past century (Shears and Bowen, 2017), making it difficult to predict how organisms like sponges will fare with climate change. Different regions of New Zealand have warmed at different rates, with the greatest warming observed in the south. Central New Zealand, by contrast, has shown little warming over the past decades, indicating that organisms across all of New Zealand may have experienced different levels of stress associated with climate change. Law et al. (2017) predicts warming across all of New Zealand within the next century. The simulations run by these authors indicate that southern New Zealand will exhibit the highest degree of warming over the next century and central New Zealand will be less affected. Therefore, those organisms existing in southern New Zealand may experience the highest levels of thermal stress and be at the highest risk of population declines, if they cannot adapt quickly enough. Expected environmental changes for central New Zealand instead may be most pronounced with regards to seasonality, as Shears and Bowen (2017) have recorded recognizable shifts in seasonality over the past fifty years. Those organisms that rely on seasonal cues for processes, therefore, may have to adapt to such changes. If changes in seasonality result in a shift in processes like asexual reproduction, population dynamics and genetic diversity of such organisms are likely to experience change as well.

Here, I investigate budding in Tethya bergquistae and T. burtoni to gain a better understanding of potential drivers of asexual reproduction. My specific aims were: (1) to characterize budding events by sampling two separate populations $5 \mathrm{~km}$ apart, determining any differences between species and sites, and identifying potential correlations with environmental variables (sea surface temperature, chlorophyll- $a$ concentrations, rainfall); (2) to characterize budding over time in the same monitored sponges and to determine any correlations with genotype or water temperature (from an in situ temperature logger); and (3) to experimentally explore the relationship between temperature and budding for both species. These results are important in that they may give an indication as to the role of asexual reproduction for these sponges and further, allow the prediction of how population growth and diversity may respond to climate change. 


\section{Methods}

\section{Sampling sponge populations}

Twenty individuals (Tethya bergquistae and T. burtoni) were collected by SCUBA from two separate sites $(\mathrm{n}=10$ individuals from Breaker Bay and 10 from Princess Bay for each species; see Chapter 5, Figure 5.2 for map of sites) every month from February 2015 - 2017 (same sponges as in Chapter 3). Despite potential cryptic speciation within T. burtoni (see Chapter 2), the group was considered as one species here as members belonging to each group within T. burtoni could not be identified based on morphology alone. Sponges were recorded as budding or not (Figure 4.1), and for those sponges that were budding, the total number of buds was counted. The diameter of each sponge was measured using calipers, and the surface area of each sponge was estimated using the surface area of a sphere $\left(V=4 \pi r^{2}\right)$. Instances of budding, as well as bud density (number of buds / surface area, as in Cardone et al., 2010) were tested for the effect of environmental covariates, including monthly means of SST $\left({ }^{\circ} \mathrm{C}\right)$, monthly means of chlorophyll-a concentration $\left(\mathrm{mg} \cdot \mathrm{m}^{-3}\right)$ and total monthly rainfall $(\mathrm{mm})$ that were collated in Chapter 3.
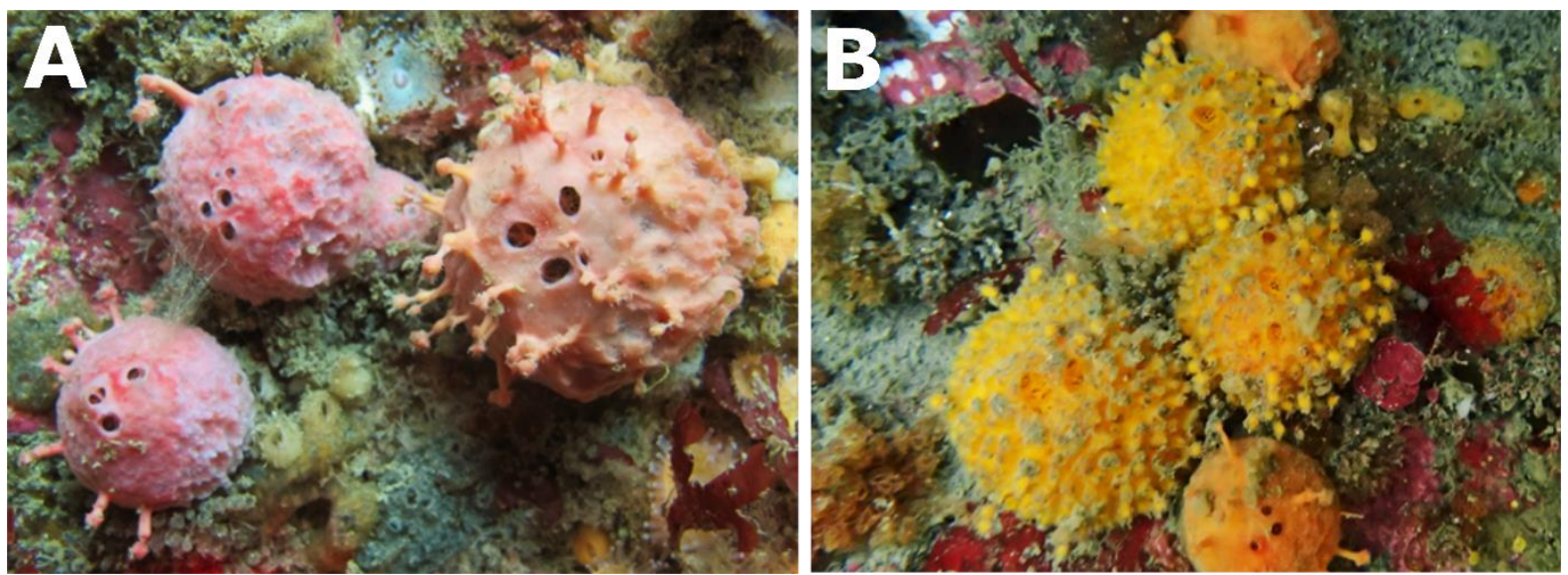

Figure 4.1. Examples of budding in Tethya bergquistae (A) and T. burtoni (B).

\section{Repeated observations on sponges}

Populations of $T$. bergquistae and T. burtoni at Breaker Bay were monitored over time by means of repeated photographs. For $T$. bergquistae, a population of about 90 sponges was photographed from November 2015 to November 2017. Six areas (approximately $2 \mathrm{~m}^{2}$ ) were haphazardly chosen within $250 \mathrm{~m}^{2}$ of rocky reef, and areas were $\sim 10-15 \mathrm{~m}$ apart. Within each 
of the six areas, random groups of three to six sponges were tagged (using sheep tags epoxied to the reef). From November 2015 - 2016, sponges were photographed every month and from November 2016, sponges were photographed six times (January, February, June, August, September, November 2017). For T. burtoni, a population of approximately 50 sponges at Breaker Bay was monitored from May 2016 to November 2017. Six additional areas were chosen within the same $250 \mathrm{~m}^{2}$ of rocky reef, and 3 tags were placed per area to correspond to two to six sponges. From May 2016 - November 2016, sponges were photographed monthly and following that, were photographed in: January, February, April, June, August, September and November 2017. For sponges within the T. burtoni complex (see Chapter 2), a tissue sample was taken from each sponge using a biopsy punch and was genotyped for species identification using a panel of 11 microsatellite markers using the methods described in Chapter 5. All sponges from photographs were recorded as budding or not, and the total number of buds for each sponge was counted in ImageJ (Schneider et al., 2012). Total number of buds was used in lieu of bud density due to the fact that sponges changed their volume over time (members of the Tethya genus contract and reduce body size up to 75\%; e.g., Nickel, 2004), which would affect the calculation of bud density for the same individual over time. Two temperature loggers (HOBO Temperature, Onset Corporation Ltd.) were deployed during the study period and recorded hourly measurements of temperature. Instances of fusion between sponges were also recorded over the monitoring period.

\section{Exploratory experiment}

Because temperature was shown to potentially influence budding events from field data (see Results), an experiment was conducted to further investigate the effect of temperature on budding for both T. bergquistae and T. burtoni, with particular interest in the acute effects of high temperatures on budding. Sponges were collected from Princess Bay in April 2017 after their annual sexual reproductive event (see Chapter 3) to avoid potential confounding results from some individuals participating in sexual reproduction and others not. Sponges were collected when the water temperature was $16-17^{\circ} \mathrm{C}$ (known from in situ temperature logger readings). Sponges were then placed onto mesh disks and allowed to attach and acclimate in a sea table with flow-through seawater for 2 weeks before the experiment began. Temperature in the sea table was monitored using a temperature logger and was $16 \pm 2{ }^{\circ} \mathrm{C}$ throughout the acclimation period and reflected temperature variation experienced in the field which is due to 
alternating southerly/northerly winds. Southerly fronts that frequent this study location result in decreases in temperature as water moves northwards from Antarctica, and thus water temperature can be variable over short periods of time.

Temperature treatments included: $17^{\circ} \mathrm{C}$ (control), $19^{\circ} \mathrm{C}$ and $21^{\circ} \mathrm{C}$. Sponges were collected at the end of summer, when seawater temperature was maximum for the year $\left(17-18^{\circ} \mathrm{C}\right)$. The upper two temperatures were chosen to examine the potential effects of climate change, meant to simulate shortened periods of thermal stress due to heat waves, as New Zealand is expected to experience a $2.5^{\circ} \mathrm{C}$ increase in sea surface temperature over the next century (Law et al., 2017). The experiment was conducted in a temperature control room maintained at $15^{\circ} \mathrm{C}$. The experimental set up for each temperature treatment included three tanks. A top header tank (70 L) received flow through of raw sea water and reduced natural temperature fluctuations in the flow-through water; this tank fed a second, intermediate header tank (70 L), which contained heaters to reach desired temperature treatments. Finally, the intermediate header tank fed water to a treatment tank $(70 \mathrm{~L})$ that contained the sponges and no heaters to minimize negative effects of direct heating. Water levels for each header system were maintained using a ballcock supply valve, which allowed water to be sufficiently heated. The flow rate to the treatment tank for sponges was $0.70 \mathrm{~L} \cdot \mathrm{min}^{-1}$ maximum (entire tank turnover within 100 min). Tethya have been found to pump $\sim 100 \mathrm{ml} \cdot \mathrm{min}^{-1}$ (Perea-Blàzquez, 2011), which means it would take 20 sponges (sample size used, see following paragraph) 35 minutes to pump through all of the water in the tank. Flow rate could not be increased beyond $0.7 \mathrm{~L} \cdot \mathrm{min}^{-1}$ because temperatures would become unstable, as water from the header tanks would not have had sufficient time to heat up. Sponges would have therefore recirculated the water $3 \mathrm{x}$ before the entire tank was turned over. To mitigate the effects of reduced flow, multiple bubblers were added to each tank to create water movement and re-oxygenate the water, and further, a $1 / 4$ volume water change was performed every day, where water was replaced with water of the same temperature water taken from header tanks. Temperature was monitored using temperature loggers (two per tank) and was maintained within $\pm 0.2^{\circ} \mathrm{C}$ throughout the experiment.

Ten sponges of each species were placed into each treatment at the beginning of the experiment, totalling 20 sponges per treatment tank. Temperature was ramped $0.5^{\circ} \mathrm{C}$ per day until 
treatment temperature was reached (as in Bennett, 2017), which then began $t_{0}$ for that treatment. A ramping of $0.5^{\circ} \mathrm{C}$ per day was considered sufficient time to allow sponges to acclimate to treatment, as temperature data from the field indicated sponges may sometimes experience temperature changes of $1-2^{\circ} \mathrm{C}$ per day (Figure 4.2). Sponges were photographed daily to monitor budding. In the event that a sponge was budding prior to placement into the experiment, the number of buds was counted at $t_{0}$ so that the change in buds (i.e., measure of bud production) over the experiment period could be measured. Buds were counted, and the diameter of each sponge was measured to estimate surface area and volume in ImageJ (Schneider et al., 2012). Dead sponges were removed from each treatment as they occurred to avoid anoxic conditions associated with tissue decomposition of the dead sponge. The experiment was run for 15 days to explore acute effects of temperature (i.e., simulating a short heat wave) on budding in Tethya. Previous observations from sponges kept in flow-through tanks revealed that bud production can happen over the scale of one day, thus 15 days was considered sufficient time to see budding events if they were going to occur. Because no bud production was observed during the monitoring period, the experiment was terminated and no further replications were run.

\section{Data analysis}

\section{Sampling sponge populations}

All of the following analyses were conducted in R v 3.5.0 (R Core Team, 2017). For sponges that were collected monthly, a generalized linear model (GLM) with a binominal family and logit link was used to determine the effects of potential explanatory variables on instances of asexual reproduction. This model tested the log-odds of the probability of asexual reproduction (i.e., 1 = budding, 0 = not budding) as a function of the following main effects: temperature, chlorophyll- $a$ concentration, rainfall, species, site and sponge size (diameter). To check that the model met the assumptions of a logistic regression (i.e., linearity, no multicollinearity of explanatory variables), a Box-Tidwell procedure with 1,000 iterations was employed (Box and Tidwell, 1962). No variables exhibited multicollinearity. Temperature failed to meet the linearity assumption, so four even categorical groups were used, where $1=<12^{\circ} \mathrm{C}(\mathrm{n}=160$ sponges $), 2=12-14^{\circ} \mathrm{C}(\mathrm{n}=280) ; 3=14-16{ }^{\circ} \mathrm{C}(\mathrm{n}=278)$ and $4=16+{ }^{\circ} \mathrm{C}(\mathrm{n}=277)$. Another model was fitted using temperature as a categorical variable, in addition to the GLM using temperature as a continuous variable, to compare the effect of temperature in both cases. 


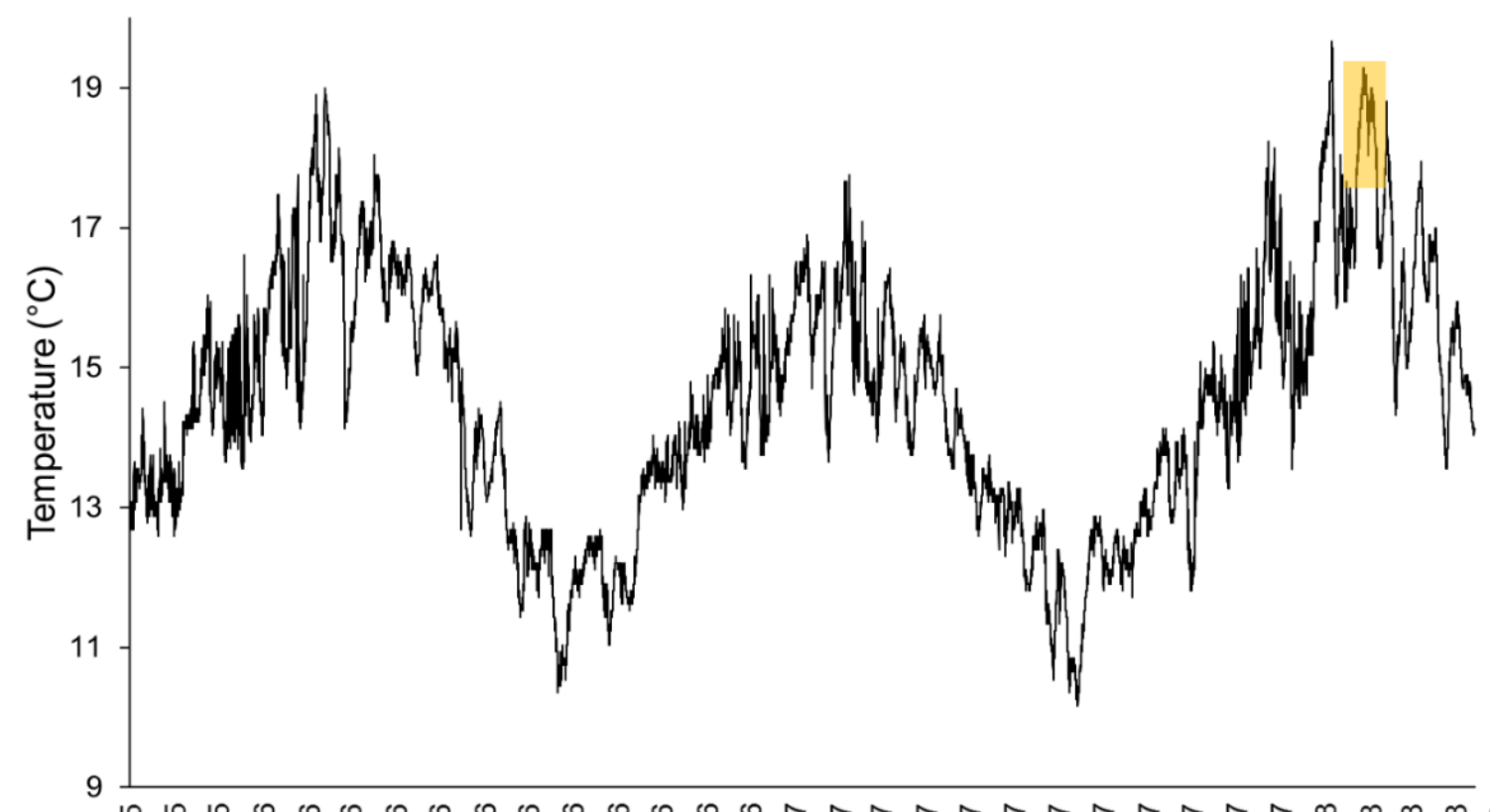

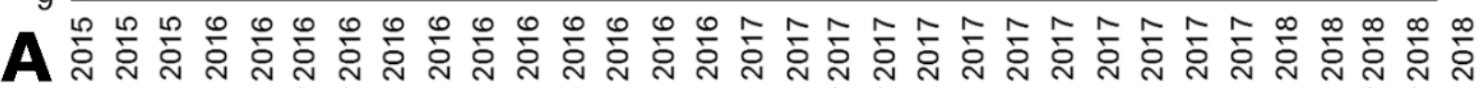

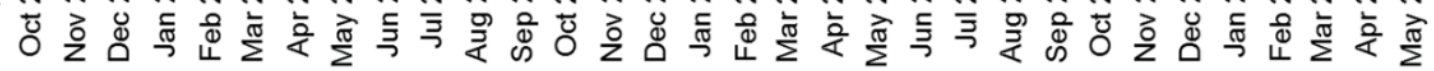

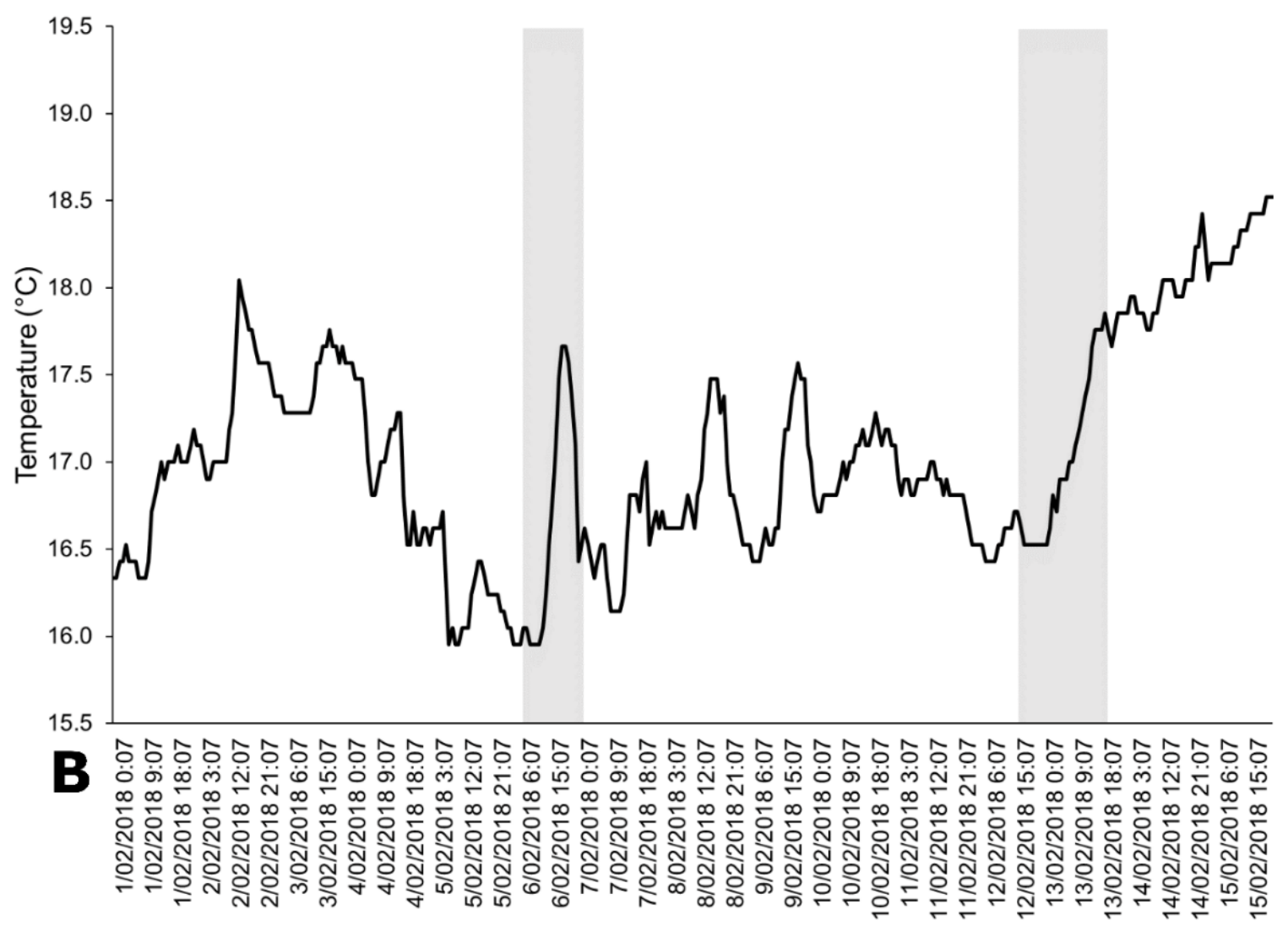

Figure 4.2. Temperature readings from deployed temperature logger at Breaker Bay from October 2015-May 2018 (A), with highlighted area enlarged in plot B. Grey bars on plot $\mathrm{B}$ highlight a change in temperature greater than $1^{\circ} \mathrm{C}$ experienced within $24 \mathrm{~h}$. 
Significance of effects were assessed using likelihood ratio $\chi^{2}$ tests. The following interactions of interest were also tested in addition to the main effects: sitexspecies, sizexspecies, temp $\times$ chl$a$, temp $\times$ rain, rain $\times$ chl- $a$, and species $\times$ each environmental variable. The best fit model was then selected by sequentially removing non-significant effects.

To determine potential correlates to asexual reproduction, a linear model was fitted to test the effects of temperature, chl- $a$, rain, site, species and sponge size to bud density, including the same interactions terms stated above. Only those sponges that had buds were used (i.e., all zero values were removed). The best fit model was selected by sequentially removing nonsignificant effects. Significance of potential predictors was assessed using Type-III ANOVAs. All data were checked for normality, homoscedasticity, and no multicolinearity/autocorrelation of independent variables, to meet the assumptions of a linear regression (Poole and O'Farrell, 1971). Bud density was log transformed to meet these assumptions and no independent variables were correlated (see Appendix 3, Figure A3 for plot of environmental data). No statistical comparisons were made between months as there were too few data points for each month for robust comparisons. Model selection for both logistic and linear models was based on significance testing rather than AIC scores as I was not concerned with the model performance for making additional predictions, but instead was interested in the rejection of the null hypotheses (Krueger, 2001; Mundry, 2011).

\section{$\underline{\text { Repeated observations on sponges }}$}

To assess the effect of temperature and genotype on both instances of budding and number of buds, generalized estimating equations (GEEs) were constructed using the package "geepack" (Halenkoh and Højsgaard, 2006) in R v 3.5.0 (R Core Team, 2017). GEEs are an extension of GLMs but allow the effects of explanatory variables to be made for longitudinal data (i.e., repeated measures), while allowing data to be non-normally distributed (i.e., binary data, count data; Zeger and Liang, 1986; Zorn, 2001; Ballinger, 2004). GEEs are marginal models, in that they allow interpretation from the population mean, as opposed to conditional models (e.g., mixed models) with allow interpretation of subject-specific responses within assigned clusters of data (Ghisletta and Spini, 2004). The use of GEEs was chosen here over the mixed model method as I was more concerned with an overall trend in the response of the population of sponges, which all shared common values of the predictor variables (e.g., predictors were 
consistent throughout all tagged sites). I was not interested in cluster-specific estimates of the probability of budding, as in the design of this study (e.g., tagged sites), sites were haphazardly chosen to represent a subpopulation of the entire population. Furthermore, predictors were chosen without consideration for differences between tagged sites (e.g., microhabitat measurements like rugosity and sedimentation were not measured), but instead were population focused (i.e., temperature), for which a GEE is more appropriate (Zorn, 2001). The data were checked to meet the assumptions for GEEs, which are: linearity between dependent variable and predictor variables (which was met using a logit link for instances of budding and a log link for bud counts); sample size (sponge) with associated longitudinal dataset to be greater than ten $\left(\mathrm{n}_{T}\right.$. bergquistae $=71, \mathrm{n}_{T}$. burtoni $\left.=28\right)$; and that observations between sponges are independent (Ghisletta and Spini, 2004).

The GEE method is advantageous in that it allows the correlation structure for the repeated measures to be specified (Ballinger, 2004). Repeated measures from the same sponge over time were accounted for in the model by using an autoregressive (AR) correlation matrix, which specifies that the response variable depends linearly on its previous value. The AR(1) correlation structure was specifically chosen, which considers only the previous term in the longitudinal data set. In this instance, presence and number of buds were considered to be correlated to the previous time point because observed buds could potentially be products of the previous observation, particularly in the case that sponges were undergoing a budding event that lasted longer than two observation points. Temperature was taken from temperature loggers and the average daily temperature was recorded for the date when the sponge was photographed, which allowed bud density to be compared to daily temperatures instead of monthly means (as in the 'Sampling sponge population' methods). As budding has been observed to occur over a short period of time, this was to elucidate the effect of temperature and determine if different trends in response to temperature emerged using both methods. Genotype was assessed using data from Chapters 2 and 5, where T. bergquistae were all considered one genotype and T. burtoni belonged to one of two groups (see Chapters 2 and 5). Because the time frame of monitoring was different for each species, each species was first assessed separately. For T. bergquistae, a GEE was fitted to determine the effect of temperature on both instances of budding and number of buds, using a binomial and Poisson family, respectively. For T. burtoni, a GEE was fitted to determine the effect of temperature and genotype on both instances of budding (family = binomial) and number of buds (family = 
Poisson). To compare both species, only those time points that both species shared were used. Two final GEEs were constructed that examined temperature and genotype (three total) on instances of budding and number of buds. Significance for all GEEs were assessed using the Wald chi-square test.

\section{Results}

\section{Sampling sponge populations}

A summary of the budding events and bud density for sponges collected monthly from February 2015 - 2017 is presented in Table 4.1 Overall, approximately 50\% of all sponges (n =995) were found to contain buds. When examining the main effects on the probability of budding, the following were significant: site, size, species and temperature (see Appendix 5, Table A5c). The best fit model fitted the probability of budding as a function of: sitexspecies, species $\times$ size, SST $\times$ chl- $a$, and chl- $a \times$ species (see Appendix 5, Table A5d). The interaction between site and species was significant $\left(\chi^{2}=10.609, P=0.001\right)$, where at Breaker Bay, Tethya bergquistae had a higher probability of containing buds compared to T. burtoni, but no difference occurred at Princess Bay (Figure 4.3). The interaction between species and size was also significant $\left(\chi^{2}=16.714, P<0.001\right)$, where the probability of budding increased with size for $T$. bergquistae only (Figure 4.4A). The influence of chl- $a$ on budding events differed between species $\left(\chi^{2}=6.525, P=0.011\right)$, where for $T$. bergquistae, chl- $a$ was negatively correlated to the probability of budding but for T. burtoni, it was positively correlated (Figure 4.4B). Lastly, the effect of temperature on probability of budding increased with increasing chlorophyll- $a$ concentration $\left(\chi^{2}=5.226, P=0.022\right.$; Figure 4.5).

When examining the influence of only the main effects on bud density, the following were significant: size, species, SST and rainfall (see Appendix 5, Table A5e). The best fit model described bud density as a function of: SST, rainfall, and sizexspecies (see Appendix 5, Table A5f). Temperature was positively correlated to bud density $\left(\mathrm{F}_{1,474}=9.3344, P=0.002\right.$; Figure 4.6AB $)$ and rainfall was also positively correlated to bud density $\left(\mathrm{F}_{1,474}=5.4, P=0.020\right.$; Figure 4.6CD). The interaction between sizexspecies was also significant $\left(\mathrm{F}_{1,474}=4.1933, P=0.041\right)$, where for both species, bud density was negatively associated with sponge size, but for $T$. burtoni smaller individuals had a higher bud density than T. bergquistae (Figure 4.7). 
Table 4.1. Summary information of collected Tethya spp. from Feb 2015-2017. Site refers to Breaker Bay (BB) or Princess Bay (PB). Number of sponges collected (N) and number of sponges containing buds (N-B) given for each site, species, and overall. Mean bud density in counts per $\mathrm{cm}^{2} \pm \mathrm{SD}$ averages for all sponges containing buds across all months.

\begin{tabular}{ccccc}
\hline Species & Site & N & N-B & $\begin{array}{c}\text { Mean bud } \\
\text { density }\end{array}$ \\
\hline Tethya & BB & 251 & 171 & $0.50 \pm 0.72$ \\
bergquistae & PB & 250 & 117 & $0.50 \pm 0.50$ \\
& Total & 501 & 288 & $0.50 \pm 0.64$ \\
\hline $\begin{array}{c}\text { Tethya } \\
\text { burtoni }\end{array}$ & BB & 244 & 93 & $1.02 \pm 1.34$ \\
complex & Total & 494 & 194 & $1.08 \pm 1.37$ \\
\hline Overall & & 995 & 482 & $0.73 \pm 1.04$ \\
\hline
\end{tabular}

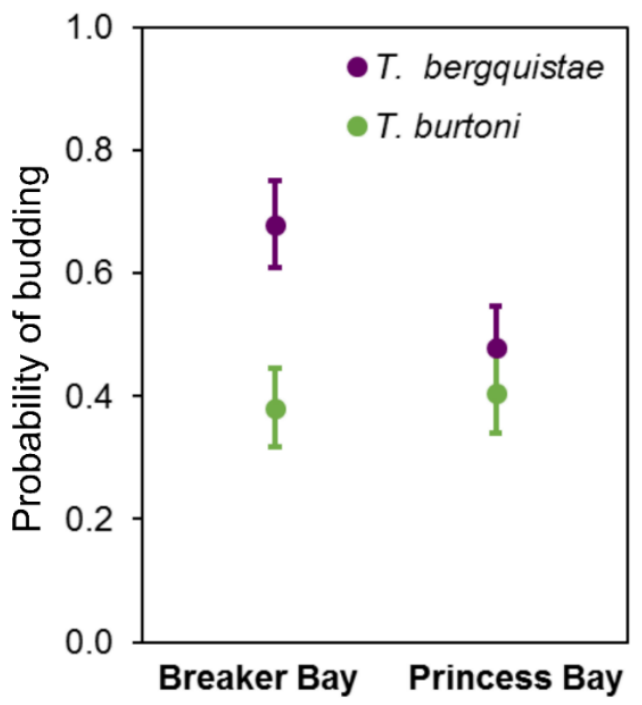

Figure 4.3. Differences in the probability of budding for Tethya bergquistae (purple) and $T$. burtoni (green) at different sites, where error bars represent $95 \%$ confidence intervals. 

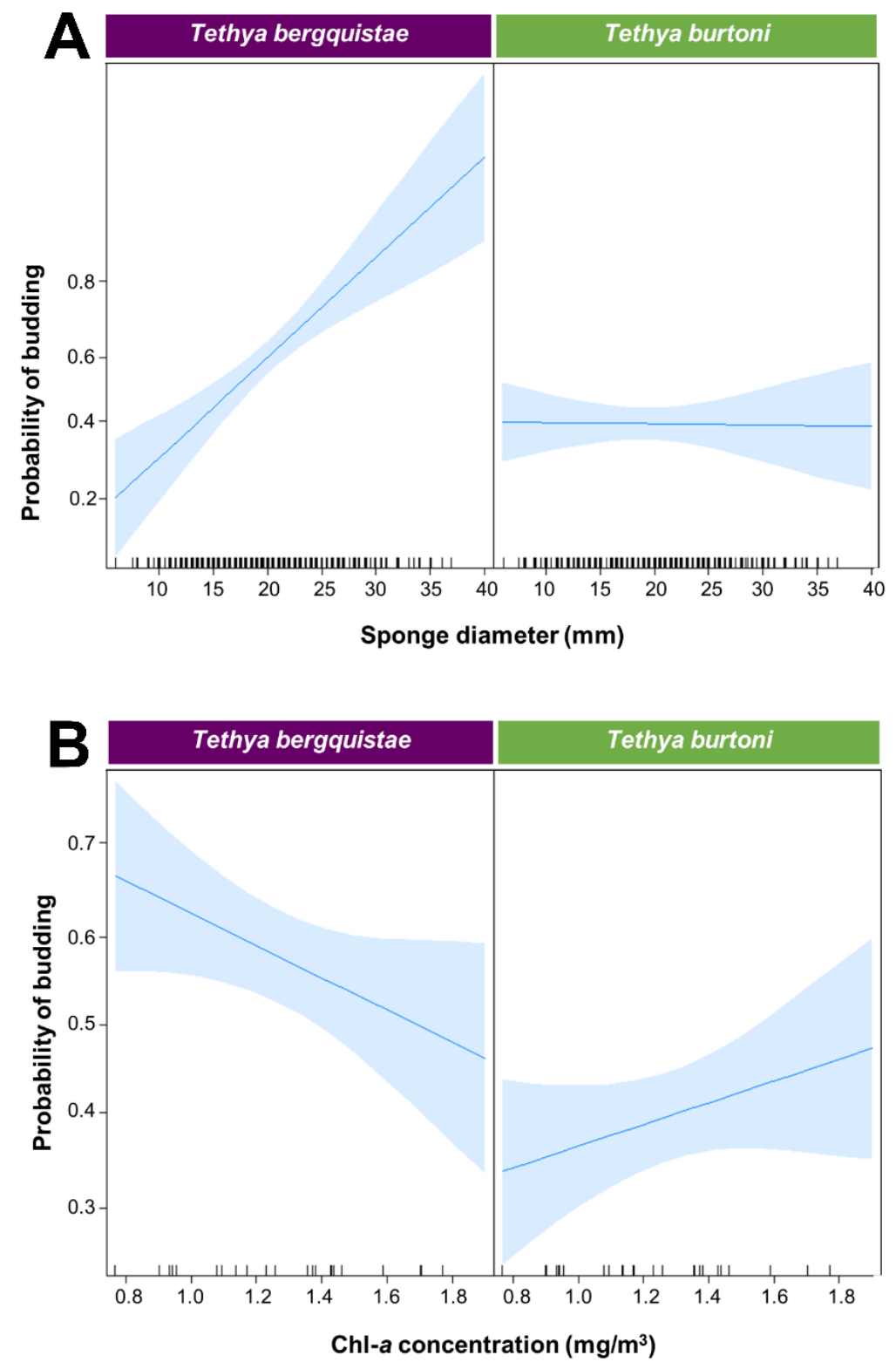

Figure 4.4. The effect of sponge size (A) and chlorophyll- $a$ concentrations (B) on Tethya bergquistae and T. burtoni. Shaded regions represent $95 \%$ confidence intervals.

\section{Repeated observations on sponges}

Monthly means of temperature readings from the HOBO logger deployed in the field were similar to readings of sea surface temperature (SST), but were slightly lower during austral summer months (Figure 4.8). In total, 129 sponges were monitored, 88 of which were Tethya bergquistae and 41 of which were T. burtoni. Over the monitoring period, mortality for $T$. bergquistae and T. burtoni was $18 \%$ and $17 \%$, respectively. Fusion events, in which two or more sponges fused together, occurred for both species (Figure 4.9). Fusion was more prevalent 

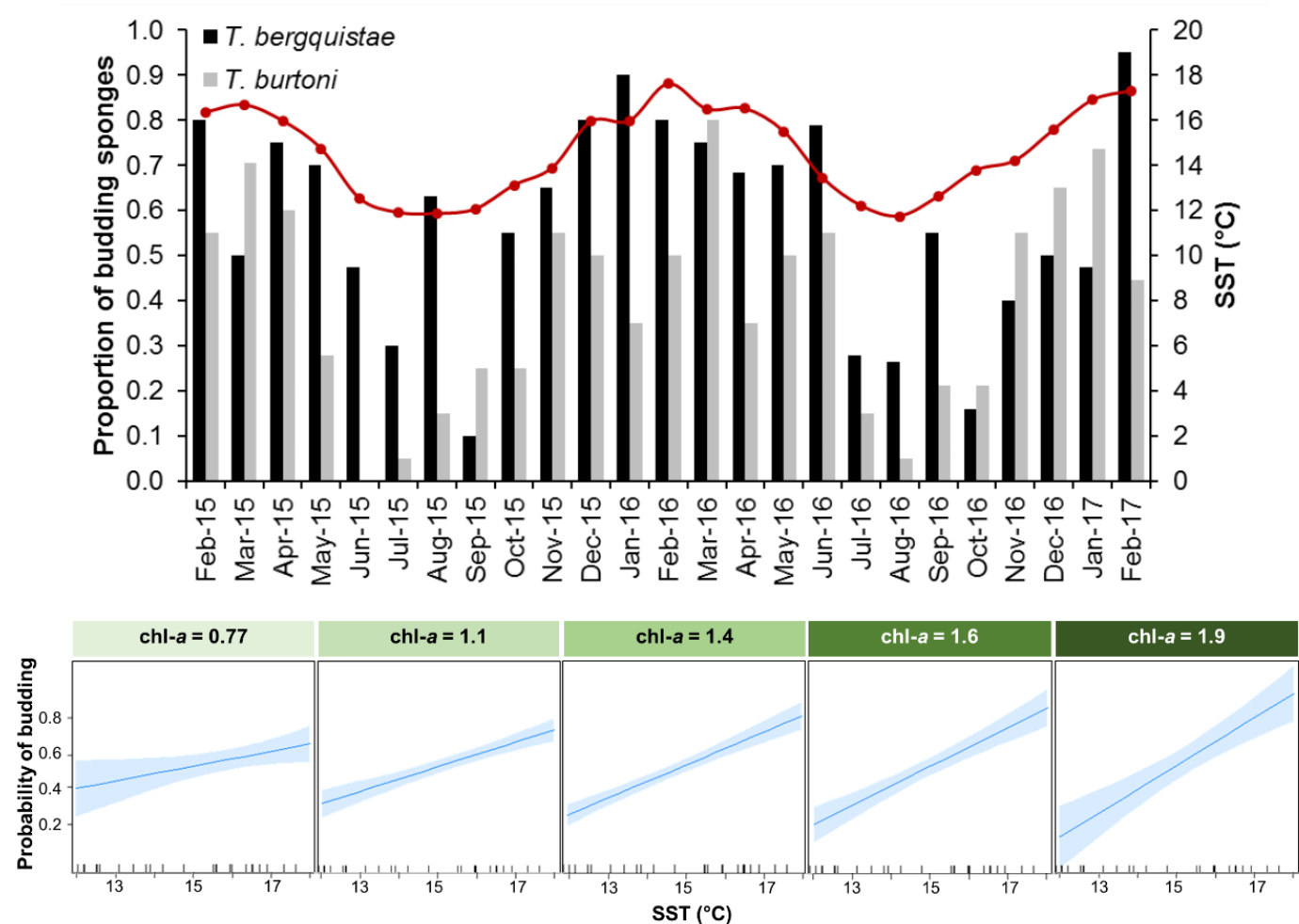

Figure 4.5. Budding events in relation to sea surface temperature (SST). Top plot presents the proportion of budding sponges collected each month, whereas bottom plot shows the effect of SST with increasing concentrations of chlorophyll- $a$ (chl- $a$ ) for both species pooled. Shaded regions on chl- $a$ plots represent $95 \%$ confidence interval.

for T. bergquistae, where 16 sponges underwent fusion (18\% of monitored population). By contrast, only two fusion events (between four sponges) were evident for T. burtoni (10\%). For both species, some individuals continually budded throughout the monitoring period, some had repeated discrete periods of budding and others did not bud at all. For plots describing the change in buds over time for each sponge, see Appendix 6. For T. bergquistae, temperature was significantly associated with both instances of budding $\left(\chi^{2}=35.5, P<0.001\right)$ and number of buds $\left(\chi^{2}=54.8, P<0.001\right)$. Similarly, for $T$. burtoni, temperature was significantly associated with the probability of budding $\left(\chi^{2}=7.34, P=0.007\right)$. There was a trend in which instances of budding were lower in one T. burtoni genotype compared to the other, though not significant $\left(\chi^{2}=0.056, P=0.056\right)$. The number of buds for $T$. burtoni was significantly positively associated with temperature $\left(\chi^{2}=22.26, P<0.0010\right)$, but genotype did not have any effect on the number of buds in T. burtoni. When both species were combined for the analysis, only temperature had a significant effect on both instances of budding $\left(\chi^{2}=49.1, P<0.001\right)$ and number of buds $\left(\chi^{2}=102.3, P<0.001\right)$. 

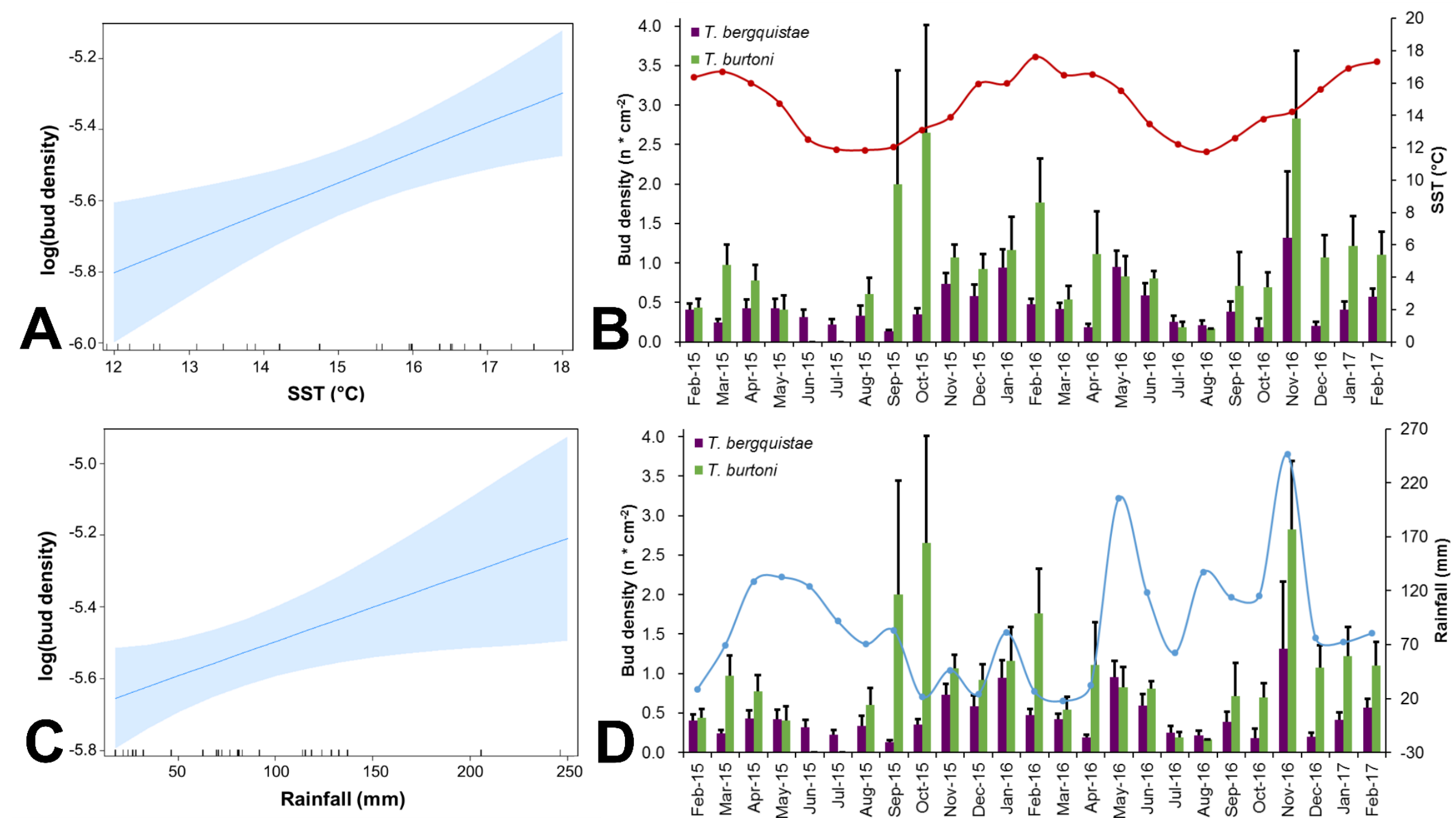

Figure 4.6. Bud density in relation to sea surface temperature (A, B) and rainfall (C, D). Monthly means of bud density for each species given (B, D), with error bars representing standard error. Shaded regions surrounding regression lines (A, C) represent $95 \%$ confidence intervals. 


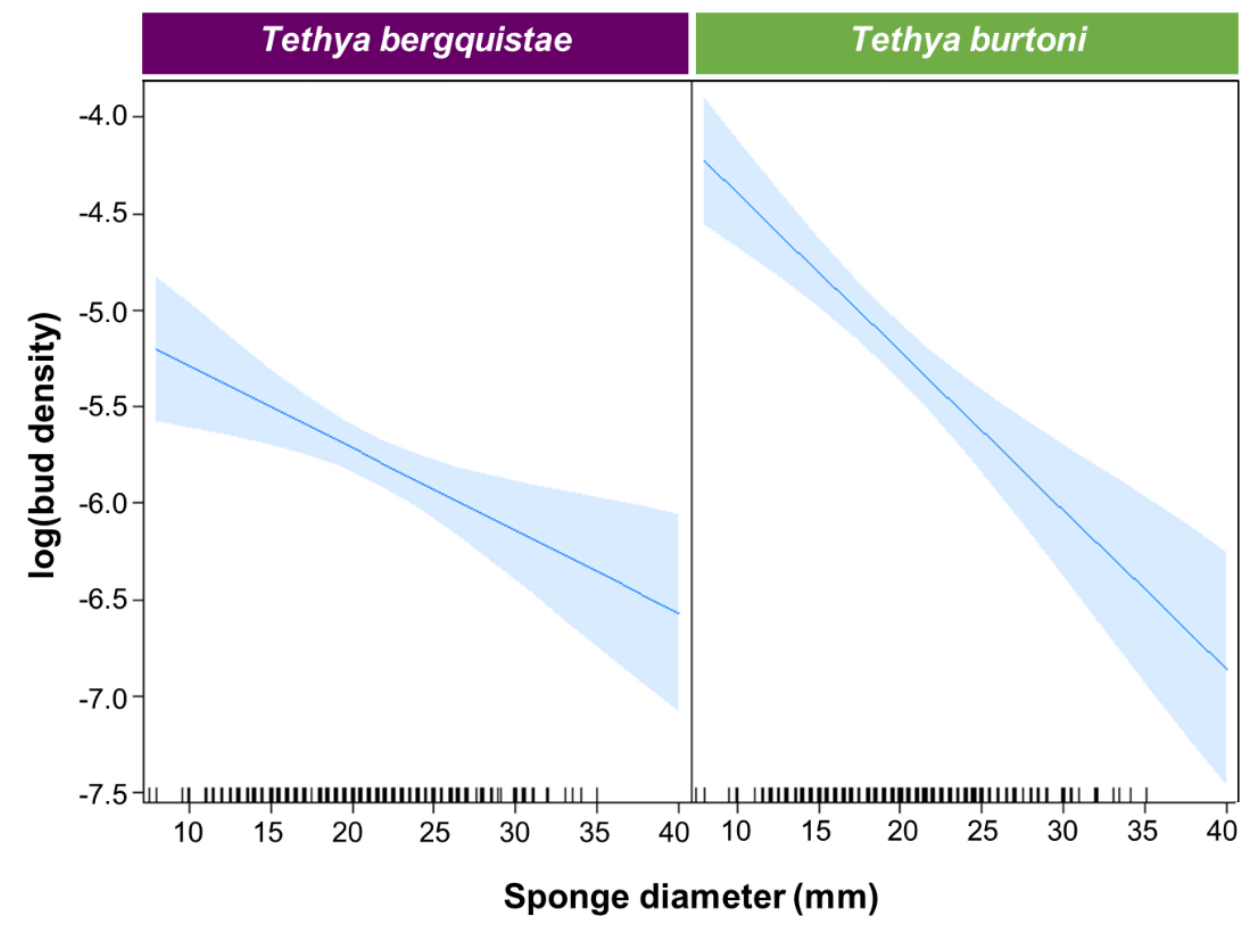

Figure 4.7. Bud density in relation to sponge size for both species, where shaded regions represent $95 \%$ confidence intervals.

\section{Exploratory experiment}

No sponges from either species produced buds for the duration of the experiment (10 of each species, per treatment; $n_{\text {total }}=60$ ). Those sponges that contained buds prior to the experiment dropped their buds within the first three days and then did not participate in asexual reproduction for the rest of the experiment. Sponge diameter remained constant throughout the experiment, and sponges revealed no signs of tissue necrosis. Mortality differed between species and between treatments. For T. bergquistae, one of the controls died on the third day (10\% mortality). There were no mortalities for $T$. bergquistae in the $19^{\circ} \mathrm{C}$ treatment $(0 \%$ mortality) and three mortalities occurred for the $21^{\circ} \mathrm{C}$ treatment half way through the treatment (30\% mortality). Mortality was higher for T. burtoni. For the control treatment, two sponges died on day 13 (20\% mortality), and both the $19^{\circ} \mathrm{C}$ and $21^{\circ} \mathrm{C}$ treatments saw nearly $100 \%$ mortality by the end of the 15 days. Differences in survivability are further described and discussed in the General Discussion (Chapter 6). 


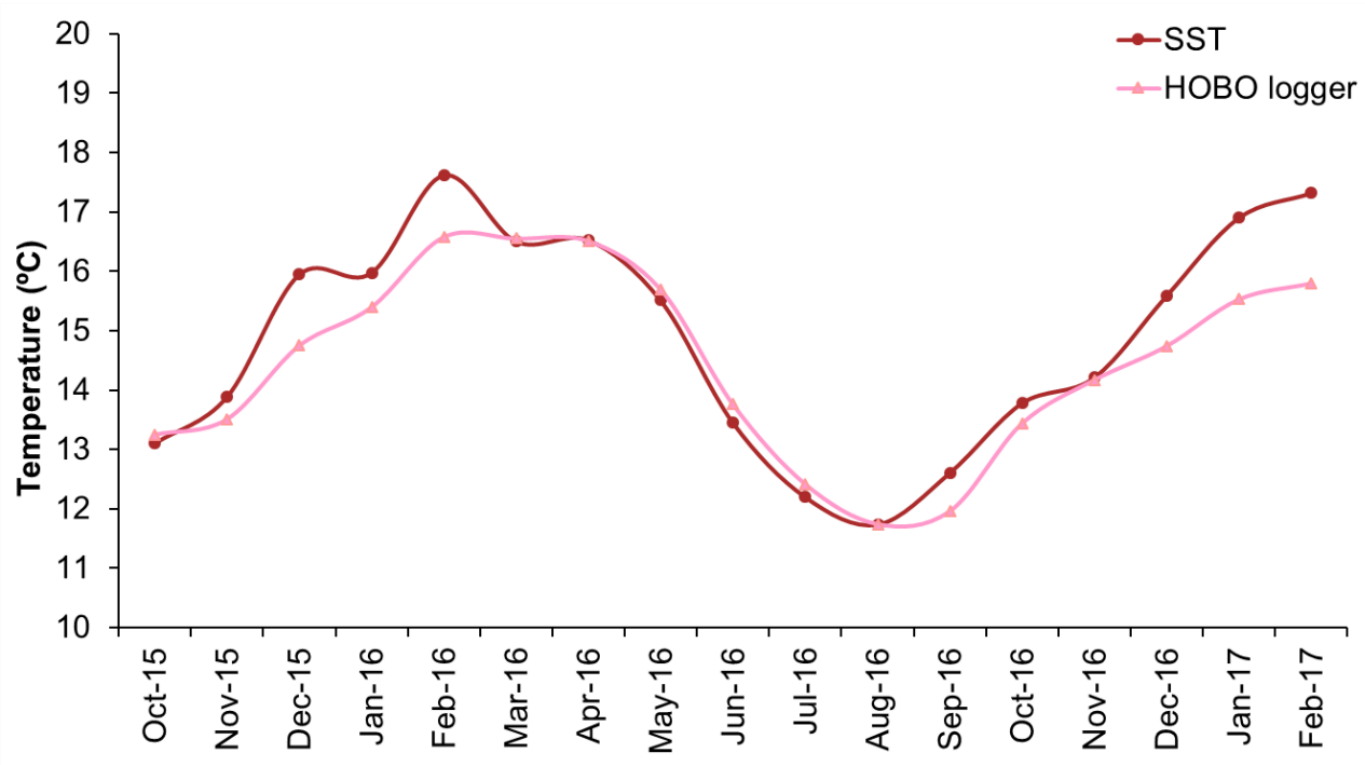

Figure 4.8. Sea surface temperature readings versus temperature readings from deployed HOBO logger.
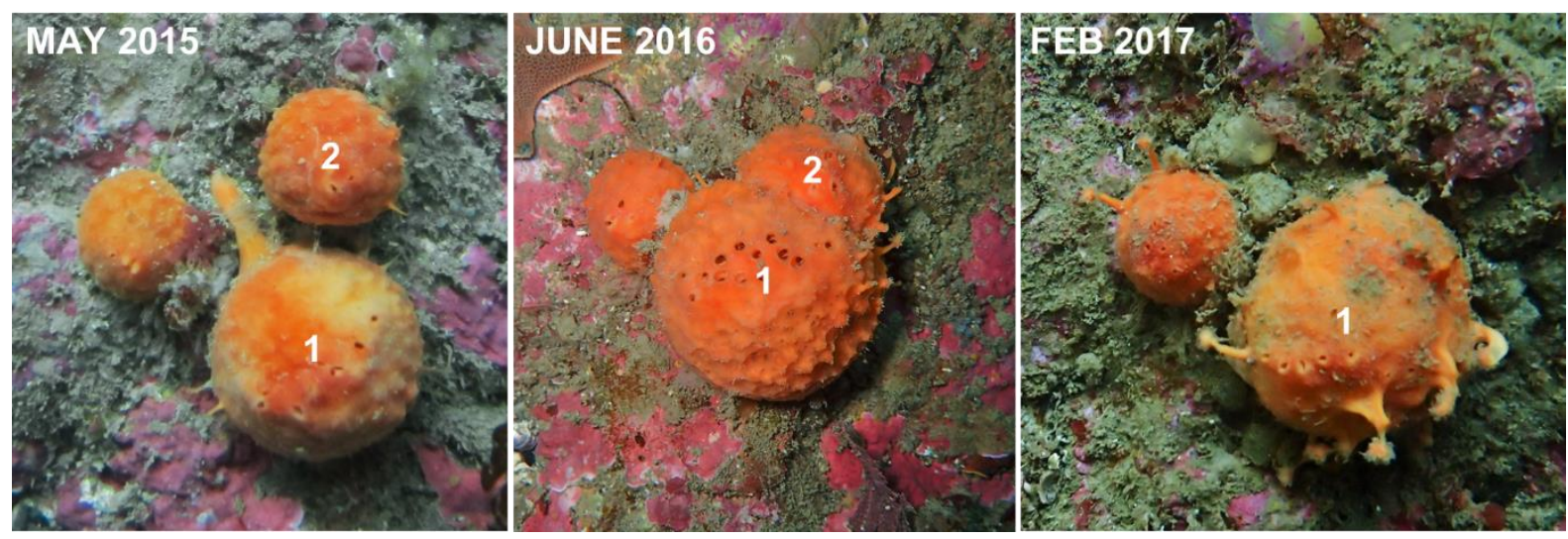

Figure 4.9. Fusion event between two Tethya bergquistae.

\section{Discussion}

This chapter used multiple methods to characterize asexual budding in Tethya spp. and determined potential environmental correlates that may be important influencers of asexual reproduction. As opposed to sexual reproduction which was an annual discrete event (see Chapter 3), budding occurred continuously throughout the year. Although continuous, there was a noticeable seasonal trend, where warmer waters were associated with both increased numbers of budding sponges and increased bud densities. These results indicate that the 
environment plays an important role in driving asexual reproduction, which is further supported by the site-specific responses of asexual reproduction observed. The experiment, which was conducted to better understand the relationship between temperature and asexual reproduction, resulted in no instances of budding, despite findings amongst in situ observations. High temperatures and associated stress may instead have resulted in the increased observed mortality. It is noteworthy, however, that the control groups displayed no instances of budding, despite asexual reproduction being common in in situ sponges as well as sponges kept previously in a flow-through sea table at similar temperatures. Such findings are likely an indication that the flow was too reduced for the experiment, resulting in increased stress and mortality. However, it may also be an indication that stable conditions do not favour asexual reproduction for Tethya spp., which, if true, suggests that asexual reproduction instead may be important for recolonization and population maintenance in disturbed environments. Overall, the findings of this chapter indicate that the timing and frequency of asexual reproduction in Tethya are likely to be altered under temperature changes predicted for New Zealand (Law et al., 2017). Further, if homogeneous environments do not favour asexual reproduction, a predicted increase in storm intensity and wave action (Law et al., 2017) may further result in an increase in asexual reproduction. While species-specific responses of budding were difficult to discern, thermal tolerance of both species differed, where T. bergquistae outperformed $T$. burtoni under thermal stress. Increases to both asexual reproduction and mortality have population level consequences and may result in a decrease in the diversity of the Tethya genus with climate change.

\section{Environmental influences on asexual reproduction}

Temperature has been shown to be associated with asexual reproduction in many marine organisms, where increased temperatures have resulted in increased asexual reproduction and in some instances, associated population growth (Littlefield et al., 1991; Purcell et al., 1999; Willcox et al., 2007). For some organisms, asexual reproduction is more prominent at environmental extremes (Snell, 1986), but extreme temperatures have also been recorded to decrease asexual reproduction and population abundance for other organisms (e.g., Yamaguchi, 1974). In this study, data from both collecting and monitoring populations in the field revealed that temperature was positively associated with both instances of budding and bud density, indicating asexual reproduction is either seasonal or may increase with warming 
waters, or both. If the latter is true, an increase in temperature could result in positive population growth. For the experiment, I chose temperatures to explore the effects of warming in a way that may simulate a heat wave or an extreme temperature event in the summer. Temperature highs of $19^{\circ} \mathrm{C}$ in the Wellington Region have been recorded (from in situ loggers) and further, Law et al. (2007) have predicted that in the next century, temperature highs will increase by $2.5^{\circ} \mathrm{C}$. The experiment, however, resulted in no budding individuals for any temperature treatments, making it more difficult to explain the exact effect of temperature on the process of asexual reproduction. Instead, it is likely that the experimental conditions of reduced flow and thermal stress resulted in increased mortality for the higher temperature treatments. While thermal stress may have resulted in the lack of asexual reproduction observed for the higher temperature treatment, the controls also showed no signs of budding, despite having a high survivability throughout the experiment. For the duration of the experiment, these sponges were subject to stable temperatures and a reduction of water flow compared to field conditions. These conditions are the opposite of what these sponges experience in situ, where they exist in an extremely dynamic environment, particularly with regard to thermal and hydrological conditions. The field temperature logger revealed that these Tethya spp. may experience increases or decreases of $2^{\circ} \mathrm{C}$ over a period of 24 hours (Figure 4.2), where frequent and alternating northerly and southerly weather patterns that shape the Wellington coast drive these temperature changes. Further, hydrological conditions are more variable as northerlies and southerlies result in periods of intense swell and periods of calmer water (Pickrill and Mitchell, 1979).

The preliminary findings from the experiment may instead indicate that asexual reproduction is driven by disturbance events and may play an important role in population maintenance and recolonization after such events. Environment heterogeneity and disturbance have been correlated to asexual reproduction for other sponges (Battershill and Bergquist, 1990) and other benthic organisms (Coffroth and Lasker, 1998; Rasheed, 2004). A further investigation into wave action and instances of budding may be needed to clarify whether stability of environment is related to budding events, but it a likely possibility for Tethya spp. Bud density was also positively correlated to rainfall, which could be further indicative that bud production is associated with storms and disturbance. Alternatively, productivity and available food that occur during the spring periods, which also experience increased rain, may explain that this correlation with rain is rather a result of the seasonality of productivity. Chlorophyll- 
$a$ concentration, which may be a proxy for food availability, influenced budding in both species differentially (discussed next), making it difficult to estimate the effect of food availability on bud densities. Nonetheless, environmental correlates suggest that changes in asexual reproduction are likely to occur with future shifts in climate (both changes in temperature and seasonality), which has implications for genetic population structure, connectivity, population growth and overall population dynamics. For instance, the positive association of budding and temperature may indicate that asexual reproduction will increase with warming oceans. This could potentially result in a higher number of clones, which may result in both a reduction of genetic diversity and an increase of inbreeding within populations (Lande and Schemske, 1985; Marriage and Kelly, 2009). Negative consequences associated with low diversity are further exemplified in a study by Williams (2001), where reduced genetic diversity in eelgrasses resulted in both a reduction of population growth and a decline in individual fitness. Tethya populations are similarly likely to experience changes in diversity due to asexual reproduction if the environment alters the frequency and overall output of budding.

\section{Differences between species: adaptive significance}

Differences in asexual reproduction between species can reveal differential adaptive strategies, and further reveal how sympatric species occupy the same niche. Reproductive isolation between $T$. bergquistae and T. burtoni was evident when examining sexual reproduction for both species (see Chapter 3). Species delineations, however, have also been described on the basis of asexual reproduction for Tethya (e.g., Bergquist and Kelly-Borges, 1991) and other organisms in general (e.g., Crespo and Pérez-Ortega, 2009). Subtle differences in asexual reproduction between species were observed in this study, but were not obvious enough to allow use of characteristics of asexual reproduction as a metric for delineating species. For instance, when sampling from two different populations, $T$. bergquistae engaged in budding significantly more than T. burtoni, but only at one site (Breaker Bay). Overall, interactive environmental influences and site-specific responses clouded any discernible differences between species that could allow species delineation. Studies of Tethya from populations in other parts of the world further support species- and site-specific patterns of asexual reproduction. For instance, both T. citrina and T. aurantium from the Mediterranean Sea display one annual period of budding (Corriero et al., 1996), compared to the continuous budding observed here for T. bergquistae and T. burtoni. Budding in T. citrina has been 
experimentally induced by subjecting sponges to increased temperature from $13^{\circ} \mathrm{C}$ to $20^{\circ} \mathrm{C}$ (Cardone et al., 2008), indicating that extreme changes in the environment may trigger asexual reproduction. Such changes were not observed with increased temperatures in this study, perhaps because the temperature increases were not as drastic or the experimental set up was too stressful. These inconsistencies in asexual reproduction across organisms of the same genus further suggest that asexual reproduction is heavily environmentally influenced and is highly species-specific, making its function across different populations difficult to assess.

The effect of sponge size on both the probability of containing buds and bud density also differed between species. Larger $T$. bergquistae were found to have a higher chance of containing buds, yet the size of T. burtoni did not correlate to budding events. Both species had a negative correlation between bud density and sponge size, and differences in bud density between species depended on the size of the sponge. Again, no obvious trends to delineate species emerged, but these differences give an indication that these congeneric sponges coexisting in the same environment may have adaptive differences. For instance, in Chapter 3, $T$. bergquistae was found to be, on average, significantly larger than $T$. burtoni. Therefore, budding may be a size regulation mechanism for this species so it does not grow too large and therefore require more resources that may otherwise be unavailable during periods of low food availability. By contrast, T. burtoni may not participate in such a regulation mechanism, as they do not grow to the same large size as T. bergquistae. Asexual reproduction has been proposed to be a size regulation mechanism for many other organisms (Sebens, 1980; Uthicke, 2001; Ryan, 2018), where multiple smaller individuals have a greater chance of getting energy/food for basic organismal functions than a single larger organism. This interaction is again supported when examining bud density, as larger $T$. bergquistae have significantly more buds than larger T. burtoni.

Interestingly, fusion was much more prominent in $T$. bergquistae. While this may be a product of species-specific responses, it could also be a product of observations, as the habitat that $T$. burtoni occupies is more sedimented and they are therefore more difficult to observe, so fusion may not have been as obvious. Nonetheless, if budding serves as a size regulation mechanism for T. bergquistae, it remains unclear as to why fusion would occur to increase sponge size. Perhaps buds fall and serve as energy stores that can fuse back to the parent sponge during 
periods of lower food availability. Such seasonally-driven size regulation by asexual means has been recorded for other sponges. For instance, Gaino et al. (2010) proposed increased fragmentation over winter periods, which allowed recolonization of the population each year. Overall, these size-related results suggest that only $T$. bergquistae may use asexual reproduction as a size regulation mechanism, further highlighting adaptive differences for these sympatric species.

Differences between species were also evident when examining the effect of chl- $a$ concentration on the probability of budding events (Figure 4.4B), where budding in $T$. bergquistae was negatively correlated to chl- $a$, but positively correlated in $T$. burtoni. Such opposing effects of chl- $a$ concentration on asexual reproduction for both species suggest that both species may have different environmental cues that induce budding. One possible explanation for the observed disparity is that both species have differential feeding strategies. For instance, perhaps $T$. burtoni feeds more heavily on picoplankton with chl- $a$ types compared to $T$. bergquistae; therefore, T. burtoni may have more available food sources and may acquire more energy that could be used for asexual reproduction. No current research has been conducted on feeding in T. burtoni to allow comparison between both species, so such a hypothesis remains mere speculation and warrants further investigation. If budding is a size regulation mechanism for $T$. bergquistae as proposed, then it may be expected that decreased nutrients (i.e., chl- $a$ ) are associated with higher instances of budding. For instance, periods of lower food availability (i.e., decreasing concentrations of chl-a) may result in sponges asexually reproducing to reduce size and thus energy requirements that could not be met with decreased available resources. A confounding result that makes interpretation of speciesspecific responses to chl- $a$ concentration challenging is that, for both species, increasing concentrations of chl- $a$ resulted in a stronger positive effect of temperature on budding events. The significant interactive effect of temperature and chl- $a$ could be a product of the seasonality of asexual reproduction, which was exemplified in the cyclical pattern of budding events observed for both species. Such interactive effects of environmental conditions on asexual reproduction have been highlighted by Purcell et al. (1999), who found that lower temperatures and food availability led to lower instances of reproduction. Such findings, however, contradict the significant species $\times$ chl- $a$ interaction observed. Instead, in situ measurement of nutrients, as well as experimental manipulation of food levels, may shed light on the differential bud response in the two species. 


\section{Conclusion}

Here, I described asexual reproduction for Tethya spp. in the Wellington south coast and find that while budding occurred continuously throughout the year, peaks in budding events and bud density occurred during periods of warmer temperature. Differences between species were not always obvious, but some species-specific responses existed. Sampling from a population over time and monitoring the same sponges were both methods that revealed temperature may be important in budding events and the overall density of buds. The experiment that investigated budding in relation to increased temperatures did not reveal the same trend, and instead indicated that $T$. bergquistae may be better adapted to thermal stress. However, lack of budding in the controls versus in situ observations suggest that perhaps changes in temperature and heterogeneous environments may be more conducive for asexual reproduction than stable conditions. Additional experiments would be needed to verify this claim, but it may be possible that asexual reproduction plays a role in population maintenance and individual sponge survivorship during repeated disturbance events. These results have important implications in terms of adaptive potential of organisms when considering how sessile marine organisms with complex reproductive strategies, like Tethya spp., may adapt to future changing oceans. 


\section{CHAPTER 5:}

DIFFERENTIAL ROLES OF ASEXUAL AND SEXUAL REPRODUCTION IN GENOTYPE SURVIVORSHIP AND CONNECTIVITY IN THE NEW ZEALAND SPONGE TETHYA BURTONI 



\section{Introduction}

Understanding connectivity in marine systems is challenging because the reproductive ecology of many marine organisms is poorly understood, barriers to gene flow are not always obvious nor consistent through time, organisms have long dispersal periods, and propagules are small and have high mortality rates (Cowen et al., 2007; Weersing and Toonen, 2009). For many sessile marine organisms, the lack of ability to move between locations and the associated competition for space has resulted in the evolution of complex reproductive strategies (Sarà, 1984). For example, invertebrates including ascidians, bryozoans, sponges and corals all reproduce sexually and asexually, which can sometimes be through hermaphroditism or even parthenogenesis (Ayre and Resing, 1986; Thomsen and Håkansson, 1995; Maldonado and Riesgo, 2008; Combosch and Vollmer, 2013; Gasparini et al., 2015). The genetic makeup of a population is largely a product of the reproductive strategy employed by an organism, among other factors which include habitat availability and environment suitability. Genetic data can offer insight into the potential roles of different reproductive modes. For example, it is generally thought that strictly sexually reproducing organisms are generally more genetically diverse than asexual ones. Reduced genetic diversity in a population, resulting from clonal reproduction or local recruitment of genotypes, can make that population prone to inbreeding depression (Ellstrand and Roose, 1987; Russo et al., 1994; Keller and Waller, 2002; Marriage and Kelly, 2009). Sexual reproduction is therefore thought to be selected for in nature because it creates genetic diversity through recombination, generating new genotypes that may support adaptation and expansion to more variable environments (Ayre, 1984; Burdon and Roelfs, 1985; Delmotte et al., 2002; Becks and Agrawal, 2010).

Asexual reproduction results in the exponential proliferation of clonal organisms, which is important for maintaining populations and allowing populations to quickly colonize an area (Shick and Lamb, 1977; Rideout, 1978; Karako et al. 2002). Barbuti et al. (2012) proposed that the main role of sexual reproduction is the creation of genotypes, while asexual reproduction serves to amplify such genotypes. Evolutionary processes at various spatial scales shape the genetic population structure at such scales. For instance, Arnaud-Haond et al. (2007) found restricted gene flow between seagrass populations across a large-scale (the Mediterranean Sea), with spatial aggregations of clonal individuals within each observed population. There is currently a lack of framework, along with no standardized methodology, for analysing genetic 
datasets that include clonal organisms (Arnaud-Haond et al. 2007), and therefore it remains challenging to find the appropriate scale to investigative desired research questions, which hinders our overall understanding of clonal reproduction. Additionally, the employment of a certain mode of reproduction may vary in different habitats. For instance, Baums et al. (2006) found predominantly asexual to purely sexual populations in a range of different geographic regions for the coral Acropora palmata, which suggests that in the future, populations may respond differently to environmental changes. Similarly, Arnaud-Hanod et al. (2006) found difference in diversity and inbreeding levels for different mangrove populations, where populations at the species range edge employed mainly asexual reproduction and were also highly inbreed. Differences in clonal strategies between environments and population spatial scale, therefore, should be considered when trying to elucidate the role of asexual reproduction in the population dynamics of an organism.

The New Zealand marine environment is characterized by a gradient of environmental conditions, from subtropical in the north, to subpolar in the south (Heath, 1981; Wilson et al., 2005). This range and diversity of flora and fauna make it a study location of interest for population structure and connectivity studies. In New Zealand, roughly sixty studies have been conducted describing the population structure of over forty different taxa, as reviewed in Ross et al. (2009) and Gardner et al. (2010). From these current studies, the most common trend that has emerged throughout the country is a north-south differentiation in genetic structure (e.g., Perna canaliculus in Apte and Gardner (2002); Amphipholis squamata in Sponer and Roy (2002); Paracorophium lucasi in Stevens and Hoggs (2004)). Many of these New Zealand organisms also exhibited an isolation by distance pattern of genetic differentiation (e.g., Perna canaliculus in Gardner et al. (1996)), where organisms with a shorter pelagic larval duration exhibited a higher degree of structure among these New Zealand populations (Ross et al., 2009). The population structure studies conducted thus far have found a high degree of differentiation within New Zealand, but there are little few studies examining population structure at a fine scale for locations existing less than $10 \mathrm{~km}$ of each other. Furthermore, there is a clear north-south differentiation for many populations, but for populations existing in central New Zealand, it is not as clear as to how these populations are related to both their northern and southern counterparts. Therefore, the need for smaller scale studies in addition to studies conducted in central New Zealand would increase our understanding of New Zealand connectivity patterns. 
Sponges are key components of benthic environments across all oceans, playing vital roles in nutrient cycling, habitat creation and water filtration (Bell, 2008). They reproduce both sexually and asexually (see Maldonado and Riesgo, 2008 for a review). Sexual reproduction occurs via gonochorism or hermaphroditism, and eggs can either be released (ovipary) or retained as brooded larvae (vivipary). Asexual reproduction occurs via fragmentation, budding or gemmulation. Both asexual and sexual reproductive timing (seasonal or continuous) and output can be influenced by environmental factors, including temperature, sedimentation, nutrients and salinity (Ayling, 1980; Fromont and Bergquist, 1994; Witte et al. 1994; Witte, 1996; Ereskovsky, 2000; Roberts et al., 2006; Riesgo and Maldonado, 2008; Gaino et al., 2010; Abdul Wahab et al., 2014). Asexual reproduction can either occur during periods when sexual reproduction has ceased (e.g., Corriero et al., 1996), or both can occur simultaneously (see Ayling, 1980). Determining the differential reliance on each mode, however, and their contribution to the genetic structure of the population remains poorly understood. With the advancement of genetic tools, a number of population connectivity studies have been conducted and increased our understanding of the extent of gene flow and the forces that shape populations for sponges (Solé-Cava and Boury-Esnault, 1999; Borchiellini et al., 2000). These studies have found that oceanographic barriers (including fronts) and topological barriers can structure populations across relatively small areas and even depths, e.g., Chaves-Fonnegra et al. (2015). However, although dispersal is generally limited over long distances, currents may aid in dispersal and result in populations being more connected across larger scales than expected (e.g. Chaves-Fonnegra et al., 2015; López-Legentil and Pawlik, 2009; Blanquer and Uriz, 2010; Riesgo et al., 2016).

Few studies to date that have considered the role of asexual reproduction in population dynamics for sponges have report mixed functions. For example, populations of the brooding Mediterranean sponge Scopalina lophyropoda contained low instances of clones, and further, clones did not have an influence on the spatial population genetic structure (Blanquer et al., 2009, Blanquer and Uriz 2010). Asexual fragments of S. lophyropoda, however, have also been found to contain active sexual entities (Maldonado and Uriz, 1999), suggesting that asexual reproduction may indirectly promote genetic diversity. However, other sponges rely more heavily on asexual reproduction. For example, Wulff (1991) found asexual reproduction to be important for population maintenance of branching sponges in the Caribbean, where fragmentation increased the genotype of the parental sponge, reducing the risk of genetic loss 
due to genetic drift and population bottlenecks. Nonetheless, there is an overall lack of information regarding the role of asexual reproduction in population maintenance, genetic structure and connectivity for sponges.

Here, I investigated the role of asexual and sexual reproduction in population connectivity and maintenance in the temperate sponge Tethya burtoni that frequently asexually reproduces by budding. I determined the extent of gene exchange between four populations in central New Zealand, and investigated the fate of the buds as well as the ability of clones to sexually reproduce and thus contribute to the gene pool of future generations. Because T. burtoni continuously asexually reproduces throughout the year and produces high densities of buds (e.g., average monthly bud densities reaching $1.55 \pm 0.56$ buds $\cdot \mathrm{cm}^{-2}$, see Chapter 4 ), it appears that asexual reproduction is persistently selected for and thus plays an important role in structuring the population. Furthermore, because the pelagic larval duration of sponges is generally short (Maldonado, 1998), local recruitment of genotypes for T. burtoni is highly likely. Due to these two characteristics (high instances of clones and local recruitment), the following genetic characteristics are expected: (1) low genetic diversity within populations (Pfeiffer et al., 2012; Li et al., 2016); (2) high levels of linkage disequilibrium for microsatellite loci within populations (De Meeûs \& Balloux, 2004); (3) high levels of inbreeding within populations (Vange 2002; Engelstädter, 2008); and (4) highly structured populations over small spatial scales, with most variation attributable to among population variance (Russo et al., 1994; Li and Ge, 2001).

\section{Materials and methods}

\section{Study species and site}

For this study, I used Tethya burtoni, a small yellow 'golf ball' sponge belonging to the second most common genus found in the waters of central New Zealand (Sarà and Sarà, 2004, Berman 2012). Tethya burtoni is gonochoristic and seasonally sexually reproduces during the austral summer (with a reproductive event from January - March, see Chapter 3), where eggs are released into the water column (ovipary). Asexual reproduction occurs continuously throughout the year, with the highest densities of buds coinciding with sexual reproduction (see Chapters 3 and 4). Tethya burtoni asexually reproduces by budding, in which small buds 
project and detach from the body of a parent sponge, and flow freely in the water before settling and growing into clones (Figure 5.1). Tethya burtoni has been identified as likely consisting of a species complex (see Chapter 2), but for this chapter, only those sponges belonging to one species (i.e., Clade 2 in Chapter 2) were used.

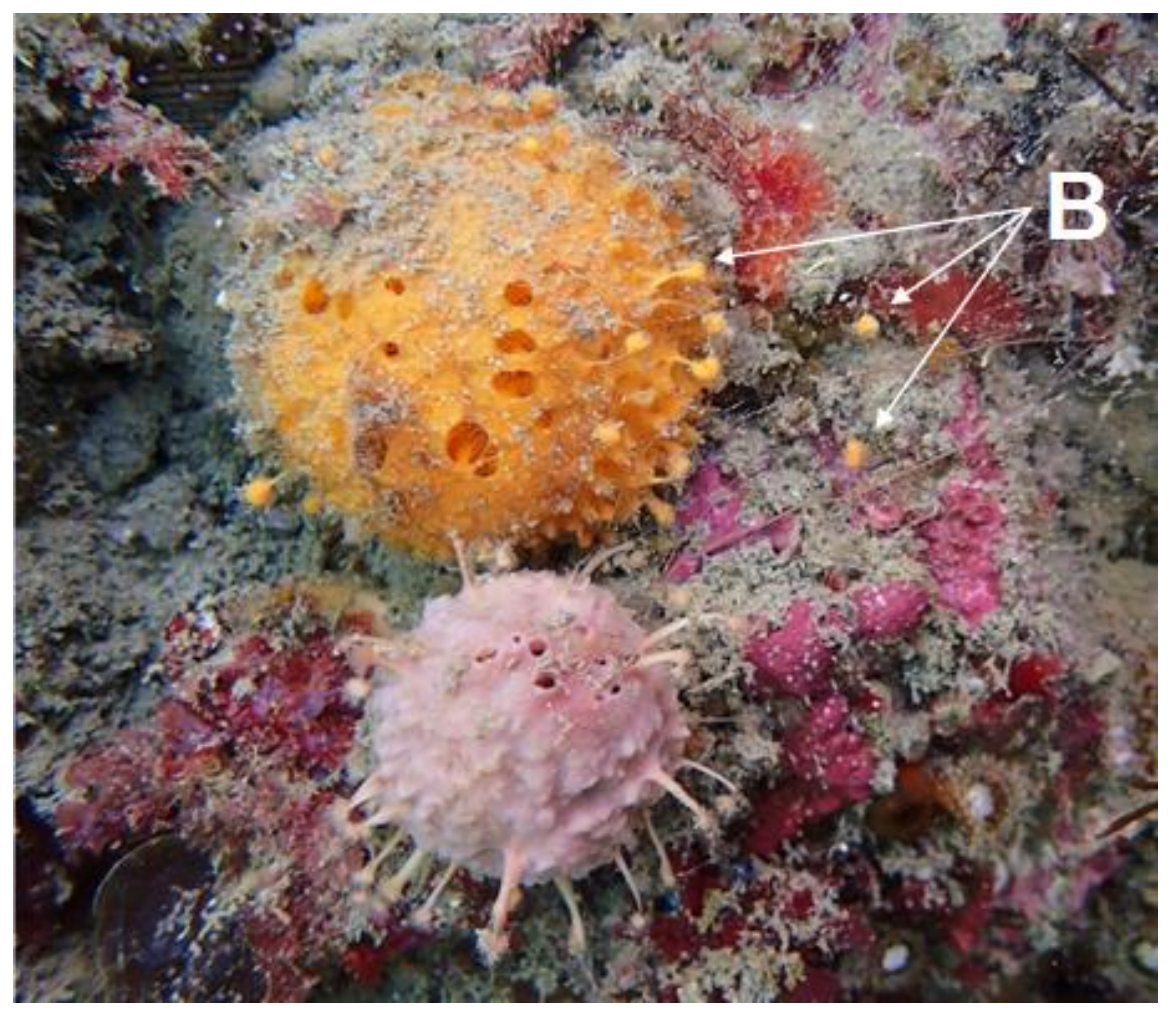

Figure 5.1. Tethya burtoni (top sponge) asexually reproducing, with buds (B) labelled.

I carried out this study at four locations in central New Zealand (Figure 5.2): Breaker Bay (BB,

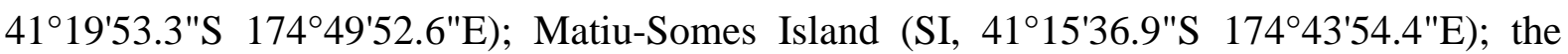
South Coast (SC, 41 ${ }^{\circ} 21^{\prime} 04.5^{\prime \prime S} 174^{\circ} 43^{\prime} 54.4^{\prime \prime} \mathrm{E}$ ); and Kapiti Island (KP, 4053'23.6"S $\left.174^{\circ} 52^{\prime} 40.3^{\prime \prime} \mathrm{E}\right)$. Three were in the Wellington region (WR), which is characterized by a highly energetic coast where strong southerly storms from Antarctica and north-westerly winds that frequently alternate and shape the coast (Harris, 1990; Carter, 2008). The WR sites included two sites exposed on the coast (Breaker Bay and the South Coast), as well as one site more sheltered in the Wellington Harbour (Matiu-Somes Island). All of these sites are within 10-15 $\mathrm{km}$ of each other. The final site was Kapiti Island, which is an island $\sim 50 \mathrm{~km}$ north of the WR. The Cook Strait current greatly influences all of these areas, and is a product of three water masses (the D'Urville, Southland and East Cape Currents) of different origins, which mix and interact to form the Cook Strait current (Heath, 1971). 


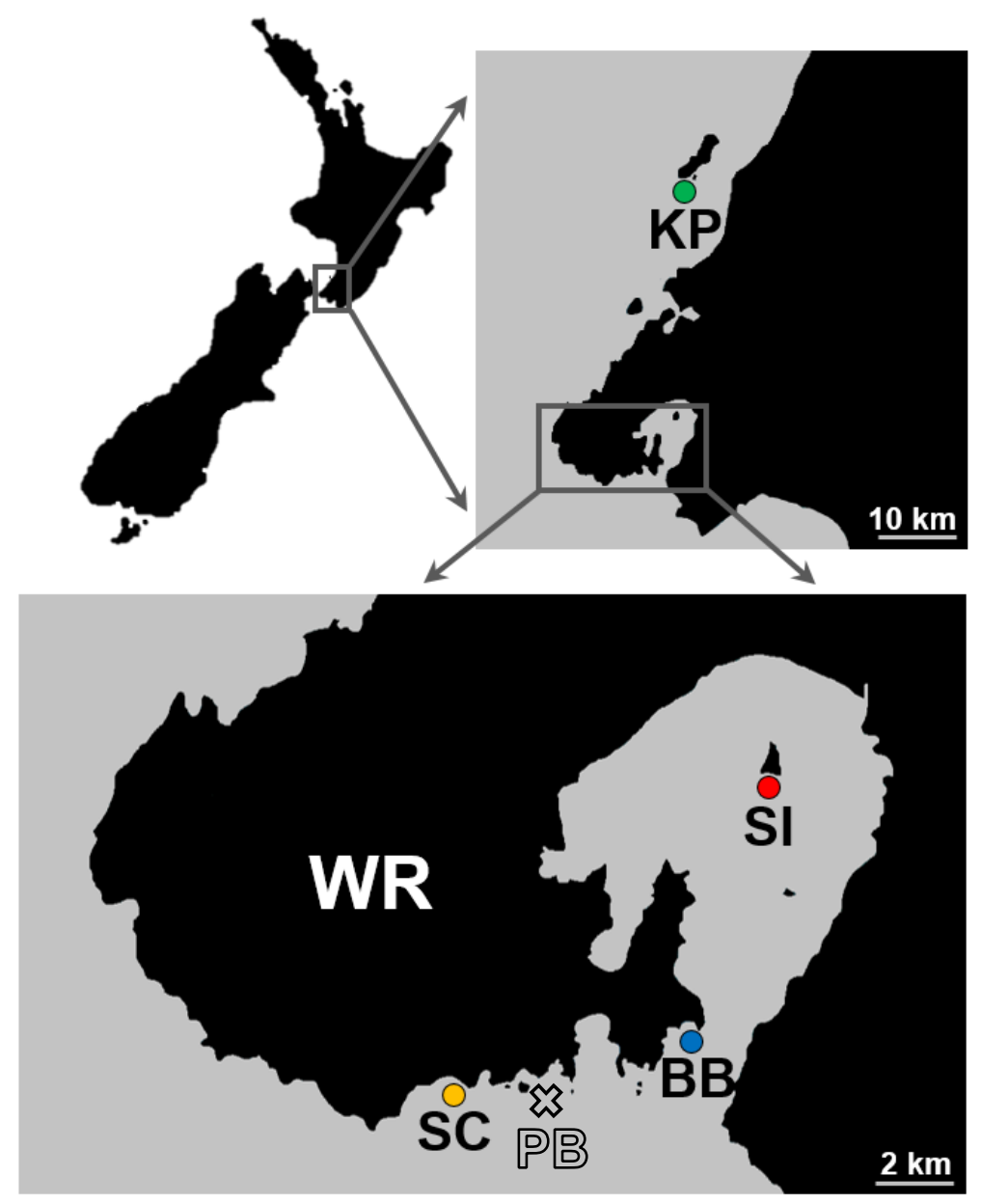

Figure 5.2. Map of study sites in central New Zealand used for this PhD thesis. Sites for population structure (Chapter 5) are indicated by coloured circles, and include Kapiti Island (KP), and three sites within the Wellington region (WR): Breaker Bay (BB), the South Coast (SC), and Matiu-Somes Island (SI). Red Rocks (sampled in Chapter 2) is the same site SC. Princess Bay (PB, sampled in Chapters 3 and 4) indicated by an $\mathrm{X}$. Scale bars given in the bottom right.

\section{Microsatellite development}

Genomic DNA was extracted from fresh tissue of five specimen T. burtoni collected from the Wellington south coast (4119'53.3"S 17449'52.6"E) using standard phenol chloroform DNA extraction methods with an additional RNA lysis step using RNase (Qiagen). Next generation sequencing was conducted using the Ion Torrent Personal Genome Machine by the Australian Genome Research Facility (University of Queensland, Brisbane, Australia). A total of 2,300,939 sequences were generated, with a mean length of $253 \mathrm{bp}$. From these, microsatellite motifs (i.e., tandem repeats) were identified using iQDD (Meglécz et al., 2009) and primers were designed based on the recommendations of Meglécz et al. (2009). 
Primers were screened on a subset of individuals $(\mathrm{n}=50)$ using multiple thermocyclers (Eppendorf Mastercycler Pro, Labnet MultiGene OptiMax Thermal Cycler, Applied Biosystems ABI 2720 Thermal Cycler). Reactions were carried out in $30 \mu 1$ reactions, with 15 $\mu 1$ MyTaq Red Mix (Bioline), $0.6 \mu$ of each primer $(10 \mu \mathrm{M}), \sim 25$ ng template DNA and a volume of distilled water to reach $30 \mu \mathrm{l}$. Amplification profiles were as follows: an initial denaturation at $94{ }^{\circ} \mathrm{C}$ for $10 \mathrm{~min} ; 35$ cycles of $94{ }^{\circ} \mathrm{C}$ for $45 \mathrm{~s}, 60{ }^{\circ} \mathrm{C}$ for $55 \mathrm{~s}, 72{ }^{\circ} \mathrm{C}$ for $60 \mathrm{~s}$; followed by a final extension at $72{ }^{\circ} \mathrm{C}$ for $10 \mathrm{~min}$. Products were visualized on a $1.5 \%$ agarose gel stained with RedSafe Nucleic Acid Staining Solution (20,000×). Thirteen loci amplified consistently and for each of these loci, the 5' end of the forward primer was tagged with one of the following fluorescent labels: FAM, TAMRA and HEX. Fluorescently labelled products were pooled, purified and genotyped using the 3730x1 DNA Analyzer (Applied Biosystems) by GeneScan Services for fragment analysis by Macrogen Inc. (Seoul, South Korea). Alleles were visualized in GeneMarker v2.2 (Hulce et al., 2011) and scored manually relative to a size standard (LIZ500). Of the thirteen loci (Table 5.1), one (TBURT13) was monomorphic and another (TBURT12) amplified in less than 50\% of the individuals, likely due to large allele dropout. These two loci were excluded, leaving a panel of eleven microsatellite markers to use for population structure analyses.

\section{Sample collection, DNA extraction and PCR amplification}

Sponges were collected using SCUBA from the four locations previously mentioned. To minimize the occurrence of clones, sponges were collected at least 3-5 m apart at all locations (following Duran et al., 2004b). Tissue for DNA extraction was immediately taken from the inside (e.g. choanosome) of the sponge and rinsed thoroughly with distilled water to minimize contamination from possible associated epibionts. In addition, at Breaker Bay, a small population of approximately 70 sponges was tagged within an area of $250 \mathrm{~m}^{2}$, sampled (where a small piece of tissue was collected with a biopsy punch) and photographed. DNA was extracted using a DNeasy Blood and Tissue Kit (Qiagen), following the instructions of the manufacturer. Eleven microsatellite loci were amplified and genotyped for each specimen by fragment analysis, following the methods described above. Alleles were visualized in GeneMarker v2.2 (Hulce et al., 2011) and scored manually relative to a size standard (LIZ500). 
Table 5.1. Microsatellite information with locus name, repeat motif, forward and reverse primer sequences (presented $5^{\prime} \rightarrow 3^{\prime}$ ), number of alleles $\left(\mathrm{N}_{\mathrm{A}}\right)$, size range of products (in base pairs), annealing temperature $\left(\mathrm{T}_{\mathrm{A}}\right.$, in $\left.{ }^{\circ} \mathrm{C}\right)$.

\begin{tabular}{|c|c|c|c|c|c|}
\hline Locus & $\begin{array}{l}\text { Repeat } \\
\text { Motif }\end{array}$ & Sequence $\left(5^{\prime} \rightarrow 3^{\prime}\right)$ & $\mathbf{N}_{\mathbf{A}}$ & $\begin{array}{c}\text { Size } \\
\text { Range } \\
\text { (bp) }\end{array}$ & Ta $\left({ }^{\circ} \mathbf{C}\right)$ \\
\hline TBURT1 & $($ acag $) 5$ & $\begin{array}{l}\text { F: TCGACACAAGTTTGGCAAGA } \\
\text { R: CTGTCCAGTGCTCAACCTGA }\end{array}$ & 11 & $150-182$ & 60 \\
\hline TBURT2 & $(\operatorname{agg}) 5$ & $\begin{array}{l}\text { F: CTGACAATACGCTGGGTGTG } \\
\text { R: CCAACCTTACGCTACCTTCG }\end{array}$ & 4 & $217-229$ & 60 \\
\hline TBURT3 & (aaac)5 & $\begin{array}{l}\text { F: ATTGTTGAAGTCCGGATGCT } \\
\text { R: ACGTAGGCTCCCTTGTTCCT }\end{array}$ & 8 & $189-213$ & 60 \\
\hline TBURT4 & (acc)6 & $\begin{array}{l}\text { F: CTCCGTAAAGCTGCTGGCTA } \\
\text { R: TACCATGGGAACTCTGCTCC }\end{array}$ & 6 & $140-176$ & 60 \\
\hline TBURT5 & (ac)11 & $\begin{array}{l}\text { F: GAGGTGAATGCCTGTTGTGA } \\
\text { R: CAGACGGATGGATCTTGTGA }\end{array}$ & 9 & $127-155$ & 60 \\
\hline TBURT6 & (aat)5 & $\begin{array}{l}\text { F: GCTTCCCAAACCACCTACAA } \\
\text { R: TTGCCATAGCGTTCAACATC }\end{array}$ & 5 & $262-271$ & 60 \\
\hline TBURT7 & (aat)5 & $\begin{array}{l}\text { F: TCTGCAAACACCTCATACGG } \\
\text { R: GTTTGAGCAACAAGGCCACT }\end{array}$ & 9 & $228-297$ & 60 \\
\hline TBURT8 & (aaac)6 & $\begin{array}{l}\text { F: GAGTGGTCCACGTTTCTTCC } \\
\text { R: ATGCTGATAATGCCCAGGAG }\end{array}$ & 9 & $131-171$ & 60 \\
\hline TBURT9 & $(\operatorname{agc}) 5$ & $\begin{array}{l}\text { F: TGGTGCTGCCACATTCTATG } \\
\text { R: GTGGCCAGTGGTAAAGGAAG }\end{array}$ & 6 & $163-169$ & 60 \\
\hline TBURT10 & (ac)11 & $\begin{array}{l}\text { F: AACCATGCTGCTCCCATAGT } \\
\text { R: CTCAGCTCGATCCATGAGAA }\end{array}$ & 14 & $112-142$ & 60 \\
\hline TBURT11 & (acat) 10 & $\begin{array}{l}\text { F: CCTGAGCAGGATCATGAGTG } \\
\text { R: AGACCCAGATTTGACCAACC }\end{array}$ & 5 & $182-222$ & 60 \\
\hline
\end{tabular}

Population genetic analyses: basic genetic diversity indices

Clones (only found at Breaker Bay) were excluded from the initial population structure analyses. Clones were identified using ${ }_{M L G} \operatorname{sim}$ (Stenberg et al., 2003), which runs simulations to determine the probability that observed multilocus genotypes in a population are a product of sexual reproduction. For each site, basic genetic diversity indices for each locus were calculated as follows. The number of alleles (NA), and observed and expected heterozygosities (Ho and He, respectively) under Hardy-Weinberg equilibrium (HWE) were calculated in Arlequin v 3.1 (Excoffier et al., 2005). The number of private alleles (Np) was determined in GenAlEx v 6.5 (Peakall and Smouse, 2012). Inbreeding coefficients ( $F_{\text {IS }}$ ) and allele richness were calculated for each population in FSTAT v 2.9.3 (Goudet, 1995). Significance of inbreeding levels was determined using randomization of alleles within samples (1000 randomizations). Significant deviations from HWE $\left(P_{\mathrm{HWE}}\right)$ by locus and population, as well as 
linkage disequilibrium for all pairs of loci, were determined in GENEPOP web v 4.2 (Raymond and Rousset 1995), with the following Markov chain parameters: 10,000 dememorization steps, 1,000 batches and 10,000 iterations per batch. All significance values were adjusted for multiple comparisons using standard Bonferroni corrections (Rice, 1989). Scoring error based on the presence of null alleles, large allele dropouts and stuttering were estimated using Microchecker v 2.3.3 (Van Oosterhout et al., 2004). Each marker was also tested for neutrality under both the infinite allele model (IAM) and the stepwise mutation model (SMM) using the software Lositan (Antao et al., 2008) with 100,000 simulations at a 99.5\% confidence interval. One locus (TBURT3) was found to be out of HWE and basic genetic diversity indices for each population were calculated again using only those loci in HWE, using the methods described above.

\section{Population genetic analyses: population differentiation}

Population differentiation was assessed using the following distance-based methods: measures of $F_{\mathrm{ST}}$, isolation by distance (IBD), and analysis of molecular variance (AMOVA). Because population differentiation based on distance is sensitive to deviations from HWE (Waples, 2015), populations were assessed using only those loci in HWE. For population differentiation, $R_{\mathrm{ST}}$ is a common measure used for microsatellites; however, the choice between traditional $F_{\mathrm{ST}}$ measures and $R_{\mathrm{ST}}$ for differentiating populations is widely debated and depends on which mutational model is most appropriate to apply to microsatellite datasets (Balloux and LugonMoulin, 2002). Measures of $R_{\mathrm{ST}}$, however, are sensitive and not informative for small sample sizes (Gaggiotti et al, 1999) and therefore $F_{\text {ST }}$ was used in this study and calculated in FSTAT v 2.9.3 (Goudet, 1995). To calculate IBD, a Mantel test with 10,000 permutations was conducted in Arlequin v 3.1 (Excoffier et al., 2005), in which a matrix of pairwise $F_{\mathrm{ST}} /\left(1-F_{\mathrm{ST}}\right)$ values was correlated to the logarithm of geographical distances $(\mathrm{km})$ between populations. Geographical distances were calculated in Google Earth as the shortest distance between locations by sea. An AMOVA with 99,999 permutations was calculated in GenAlEx v 6.5 from a distance matrix (Peakall \& Smouse, 2012) to determine whether the source of variation could be attributed to among regions (e.g. WR versus Kapiti), among populations or within populations. 
Population structure was also assessed using the following cluster analyses: Discriminant Analysis of Principal Components (DAPC) and STRUCTURE. DAPC analyses were carried out in the adegenet package (Jombart, 2008) in R (R Core Team, 2017). DAPC is a multivariate statistical approach that first transforms data with a principal coordinate analysis (PCA) and then clusters data using discriminant analysis (DA) (Jombart et al., 2010). If unknown, clusters are inferred using sequential K-means and the optimal clustering solution is selected based on having the lowest Bayesian information criterion (BIC). However, here clusters were inferred from prior group assignment based on sampling location. The DAPC method is preferable to PCA as it maximizes differences between groups while minimizing variation within clusters, as opposed to traditional PCA methods which focus on the entire genetic variation.

STRUCTURE analyses were carried out in STRUCTURE v 2.3.2 (Pritchard et al., 2000). STRUCTURE is a Bayesian clustering analysis for multilocus genotype data (Pritchard et al., 2000). This software uses an admixture model with correlated allele frequencies to probabilistically assign individuals to clusters, allowing the inference of population structure (Pritchard et al., 2000). In the STRUCTURE plot output, each individual corresponds to an individual line, which is divided into $\mathrm{K}$ segments corresponding to the probability of finding that individual in each $\mathrm{K}$ cluster. The optimal $\mathrm{K}$ value is determined in Structure Harvester (Earl and VonHoldt, 2012), which plots the likelihood values (e.g. L(K)) and $\Delta \mathrm{K}$ across multiple runs, and allows a value of $\mathrm{K}$ to be selected that best reflects the true number of populations (Evanno et al., 2005). For STRUCTURE, putative population origin for each individual was provided, and 100,000 Markov Chain Monte Carlo (MCMC) iterations and a burn in of 10,000 iterations were used. Ten replicates of each run were made, setting the K values (i.e., predicted number of genetic clusters) from 1 to 8 . For this dataset, $\mathrm{K}=3$ was the best fit (from Structure Harvester, Earl and VonHoldt, 2012) and the 10 replications for K = 3 were merged in CLUMPP (Jakobsson and Rosenberg, 2007). Plots were visualized in MS Excel (2016).

\section{Asexual reproduction}

For the sponges tagged at Breaker Bay, sponges were genotyped and instances of clones

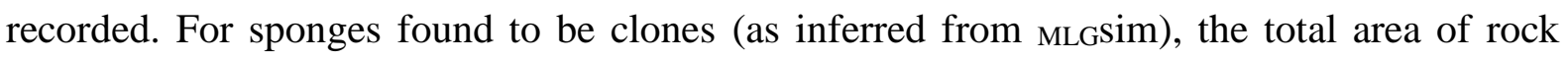
occupied by sponges belonging to the same clone group was determined using ImageJ 
(Schneider et al., 2012). The tagged clones were photographed from June 2015 to November 2017 to determine whether or not clones asexually reproduced. To examine if clones were sexually reproducing, tissue was sampled and surveyed for gametes from those clones still living after the monitoring period (13 sponges) in March 2018, the end of the annual sexual reproductive event (for histological methods, see Chapter 3). Ten other individuals were collected 3-5 m apart as a control group, to determine if the proportion of sexually reproducing sponges was different between groups. These sponges were occupying the rock by themselves (no surrounding potential clones) and were therefore assumed not to be a part of a clonal group. Based on the genotypes recorded for the sponges collected 3-5 $\mathrm{m}$ apart in the population structure analyses, these sponges were all assumed to be different genotypes. The diameter of each sponge (in $\mathrm{mm}$ ) was measured. A size frequency distribution of sexually reproducing and non-reproducing sponges was made from reproductive histological data compiled from 10 sponges collected per month from 2015 - 2017 from Breaker Bay (see Chapter 3), in combination with data from the control group, with size classes broken for every $\mathrm{mm}$. I also recalculated basic genetic diversity indices by locus and population for Breaker Bay, and recalculated $F_{\mathrm{ST}}$ and an AMOVA, but included clones in the analysis to allow for an understanding of the effect that clones may have on genetic structure. I also reconstructed DAPC and STRUCTURE plots with the dataset including clones. Methods for genetic analyses including clones are the same as described above.

\section{Results}

\section{Genetic diversity indices}

There were four different multilocus genotypes (MLGs) found (see Appendix 7). For each group of MLGs, the probability that the MLGs were a product of sexual reproduction was $P=$ $8.55 \times 10^{-14}$ or less. Genetic diversity indices by locus and by population are summarized in Tables 5.2 and 5.3, respectively. The number of alleles ranged from 2 to 11 across all loci. The locus TBURT3 showed the presence of null alleles and was out of HWE. No pairs of loci exhibited linkage disequilibrium. All loci were neutral when tested against both the IAM and SMM. When examining diversity indices by population, the mean number of alleles ranged from $3.27-4.73$ and heterozygosity ranged from $0.42-0.56$. When using all loci, the Kapiti Island population was out of HWE; however, when removing TBURT3, all populations were in HWE. 
Table 5.2. Basic genetic diversity indices for all loci for each population of Tethya burtoni, with sample size given after each population name.

For each locus, the number of alleles $(\mathrm{Na})$, observed heterozygosity $(\mathrm{Ho})$, expected heterozygosity $(\mathrm{He})$, inbreeding coefficient $\left(F_{\mathrm{IS}}\right)$, and the $\mathrm{P}-$ value for deviations from Hardy-Weinberg equilibrium $\left(P_{\mathrm{HWE}}\right)$ are given. Values in parentheses are for the Breaker Bay population including clones. Bolded and starred $P$-values indicate significance. All $P$-values adjusted using standard Bonferroni correction.

\begin{tabular}{|c|c|c|c|c|c|c|c|c|c|c|c|c|c|c|c|c|c|c|c|c|}
\hline \multirow[b]{2}{*}{ Locus } & \multicolumn{5}{|c|}{ Breaker Bay, $n=22(32)$} & \multicolumn{5}{|c|}{ Kapiti Island, $\mathbf{n = 3 0}$} & \multicolumn{5}{|c|}{ Matiu-Somes Island, $\mathbf{n = 2 6}$} & \multicolumn{5}{|c|}{ South Coast, $n=13$} \\
\hline & $\mathbf{N a}$ & Ho & He & $F_{\text {IS }}$ & $P_{\text {HWE }}$ & $\mathbf{N a}$ & Ho & He & $F_{\text {IS }}$ & $P_{\text {HWE }}$ & $\mathbf{N a}$ & Ho & He & $F_{\text {IS }}$ & $P_{\text {HWE }}$ & $\mathbf{N a}$ & Ho & He & $F_{\text {IS }}$ & $P_{\text {HWE }}$ \\
\hline TBURT1 & 4 & $\begin{array}{c}0.381 \\
(0.548)\end{array}$ & $\begin{array}{c}0.410 \\
(0.490)\end{array}$ & $\begin{array}{c}0.072 \\
(-0.121)\end{array}$ & $\begin{array}{c}0.417 \\
(0.215)\end{array}$ & 4 & 0.267 & 0.246 & -0.084 & 1.000 & 2 & 0.077 & 0.075 & -0.020 & 1.000 & 5 & 0.462 & 0.560 & 0.182 & 0.077 \\
\hline TBURT2 & 3 & $\begin{array}{c}0.500 \\
(0.424)\end{array}$ & $\begin{array}{c}0.554 \\
(0.506)\end{array}$ & $\begin{array}{c}0.099 \\
(0.138)\end{array}$ & $\begin{array}{c}0.853 \\
(0.628)\end{array}$ & 2 & 0.333 & 0.427 & 0.223 & 0.378 & 2 & 0.308 & 0.510 & 0.401 & 0.056 & 3 & 0.385 & 0.557 & 0.318 & 0.261 \\
\hline TBURT3 & 4 & $\begin{array}{c}0.273 \\
(0.250)\end{array}$ & $\begin{array}{c}0.665 \\
(0.669)\end{array}$ & $\begin{array}{c}0.596 \\
(0.630)\end{array}$ & $\begin{array}{c}\mathbf{0 . 0 0 0}^{*} \\
\left(0.000^{*}\right)\end{array}$ & 6 & 0.167 & 0.728 & 0.774 & $0.000 *$ & 3 & 0.143 & 0.589 & 0.762 & $0.000 *$ & 4 & 0.538 & 0.606 & 0.116 & 0.260 \\
\hline TBURT7 & 2 & $\begin{array}{c}0.455 \\
(0.563)\end{array}$ & $\begin{array}{c}0.359 \\
(0.411)\end{array}$ & $\begin{array}{c}-0.273 \\
(-0.378)\end{array}$ & $\begin{array}{c}0.538 \\
(0.070)\end{array}$ & 3 & 0.467 & 0.532 & 0.124 & 0.147 & 2 & 0.040 & 0.040 & 0.000 & 1.000 & 5 & 0.385 & 0.406 & 0.055 & 0.521 \\
\hline TBURT8 & 5 & $\begin{array}{c}0.773 \\
(0.781)\end{array}$ & $\begin{array}{c}0.631 \\
(0.611)\end{array}$ & $\begin{array}{c}-0.231 \\
(-0.284)\end{array}$ & $\begin{array}{c}0.474 \\
(0.074)\end{array}$ & 7 & 0.400 & 0.405 & 0.011 & 0.791 & 5 & 0.652 & 0.544 & -0.204 & 0.670 & 4 & 0.538 & 0.618 & 0.134 & 0.357 \\
\hline TBURT9 & 3 & $\begin{array}{c}0.429 \\
(0.516)\end{array}$ & $\begin{array}{c}0.669 \\
(0.670)\end{array}$ & $\begin{array}{c}0.365 \\
(0.233)\end{array}$ & $\begin{array}{c}0.056 \\
(\mathbf{0 . 0 0 1} *)\end{array}$ & 3 & 0.250 & 0.337 & 0.262 & 0.063 & 3 & 0.346 & 0.541 & 0.364 & 0.102 & 3 & 0.462 & 0.643 & 0.291 & 0.448 \\
\hline TBURT4 & 3 & $\begin{array}{c}0.227 \\
(0.375)\end{array}$ & $\begin{array}{c}0.210 \\
(0.315)\end{array}$ & $\begin{array}{c}-0.082 \\
(-0.194)\end{array}$ & $\begin{array}{c}1.000 \\
(0.631)\end{array}$ & 4 & 0.571 & 0.573 & 0.004 & 0.891 & 3 & 0.346 & 0.386 & 0.105 & 0.424 & 3 & 0.231 & 0.283 & 0.191 & 0.234 \\
\hline TBURT5 & 7 & $\begin{array}{c}0.818 \\
(0.844)\end{array}$ & $\begin{array}{c}0.786 \\
(0.745)\end{array}$ & $\begin{array}{c}-0.041 \\
(-0.134)\end{array}$ & $\begin{array}{c}0.522 \\
(0.228)\end{array}$ & 8 & 0.600 & 0.582 & -0.032 & 0.118 & 4 & 0.538 & 0.624 & 0.139 & 0.190 & 7 & 0.846 & 0.772 & -0.100 & 0.870 \\
\hline TBURT6 & 4 & $\begin{array}{c}0.810 \\
(0.625)\end{array}$ & $\begin{array}{c}0.710 \\
(0.621)\end{array}$ & $\begin{array}{c}-0.145 \\
(-0.039)\end{array}$ & $\begin{array}{c}0.007 \\
(0.017)\end{array}$ & 3 & 0.733 & 0.501 & -0.475 & 0.012 & 4 & 0.520 & 0.531 & 0.022 & 0.384 & 4 & 0.538 & 0.717 & 0.257 & 0.015 \\
\hline TBURT10 & 11 & $\begin{array}{c}0.818 \\
(0.875)\end{array}$ & $\begin{array}{c}0.840 \\
(0.841)\end{array}$ & $\begin{array}{c}0.027 \\
(-0.040)\end{array}$ & $\begin{array}{c}0.851 \\
(0.014)\end{array}$ & 9 & 0.700 & 0.652 & -0.075 & 0.932 & 6 & 0.769 & 0.739 & -0.042 & 0.675 & 8 & 0.769 & 0.858 & 0.108 & 0.107 \\
\hline TBURT11 & 2 & $\begin{array}{c}0.045 \\
(0.031)\end{array}$ & $\begin{array}{c}0.045 \\
(0.031)\end{array}$ & $\begin{array}{c}0.000 \\
(0.000)\end{array}$ & $\begin{array}{c}1.000 \\
(1.000)\end{array}$ & 3 & 0.167 & 0.159 & -0.051 & 1.000 & 2 & 0.077 & 0.075 & -0.020 & 1.000 & 3 & 0.077 & 0.218 & 0.657 & 0.040 \\
\hline
\end{tabular}


When using only those loci in HWE, the inbreeding coefficients were slightly negative for both Kapiti Island $\left(F_{\mathrm{IS}}=-0.017\right)$ and Breaker Bay $\left(F_{\mathrm{IS}}=-0.008\right)$; and positive for the South Coast $\left(F_{\mathrm{IS}}=0.173\right)$ and Matiu-Somes Island $\left(F_{\mathrm{IS}}=0.098\right)$. The South Coast population exhibited significant inbreeding when using all loci; however, after removing TBURT3, no populations showed significant inbreeding.

Table 5.3. Basic genetic diversity indices for each population, which includes: the number of samples per population (N), the average number of alleles in each population (mean NA), the average number of alleles after rarefaction to account for small sample size (mean NA after rarefaction), the number of private alleles (NPA), inbreeding coefficients $\left(F_{\text {IS }}\right)$, significance for inbreeding $\left(P-F_{\mathrm{IS}}\right)$, observed heterozygosity (Ho), expected heterozygosity $(\mathrm{He})$, and significance for deviations from Hardy-Weinberg equilibrium $\left(P_{\mathrm{HWE}}\right)$. NS indicates not significant, and $* *$ indicates significance $(P<0.0001)$. Populations are as follows: Breaker Bay excluding clones $(\mathrm{BB}-\mathrm{C})$ and including clones $(\mathrm{BB}+\mathrm{C})$, Kapiti Island $(\mathrm{KP})$, Matiu-Somes Island (SI), and South Coast (SC). Numbers without parentheses are for analyses including all 11 microsatellite loci, and numbers in parentheses are for analyses including only loci in HWE.

\begin{tabular}{|c|c|c|c|c|c|c|c|c|c|}
\hline Pop & $\mathbf{N}$ & $\begin{array}{c}\text { Mean } \\
\text { NA }\end{array}$ & $\begin{array}{c}\text { Mean NA } \\
\text { after } \\
\text { rarefaction }\end{array}$ & NPA & $F_{\text {IS }}$ & $\begin{array}{c}P- \\
F_{\text {IS }}\end{array}$ & Ho & $\mathrm{He}$ & $P_{\text {HWE }}$ \\
\hline $\mathrm{BB}-\mathrm{C}$ & 22 & $\begin{array}{c}4.36 \\
(4.40)\end{array}$ & $\begin{array}{l}3.86 \\
(3.88)\end{array}$ & $\begin{array}{c}2 \\
(2)\end{array}$ & $\begin{array}{c}0.061 \\
(-0.008)\end{array}$ & $\begin{array}{l}\mathrm{NS} \\
(\mathrm{NS})\end{array}$ & $\begin{array}{c}0.503 \\
(0.525)\end{array}$ & $\begin{array}{c}0.533 \\
(0.522)\end{array}$ & $\begin{array}{l}\text { NS } \\
\text { (NS) }\end{array}$ \\
\hline $\mathrm{BB}+\mathrm{C}$ & 32 & $\begin{array}{c}4.36 \\
(4.46)\end{array}$ & $\begin{array}{c}3.64 \\
(4.52)\end{array}$ & $\begin{array}{c}2 \\
(2)\end{array}$ & $\begin{array}{c}0.008 \\
(-0.072)\end{array}$ & $\begin{array}{l}\text { NS } \\
(\mathrm{NS})\end{array}$ & $\begin{array}{c}0.533 \\
(0.562)\end{array}$ & $\begin{array}{c}0.538 \\
(0.524)\end{array}$ & $\begin{array}{c}* * \\
(* *)\end{array}$ \\
\hline $\mathrm{KP}$ & 30 & $\begin{array}{c}4.73 \\
(4.60)\end{array}$ & $\begin{array}{l}3.81 \\
(3.65)\end{array}$ & $\begin{array}{c}7 \\
(5)\end{array}$ & $\begin{array}{c}0.096 \\
(-0.017)\end{array}$ & $\begin{array}{l}\text { NS } \\
(\mathrm{NS})\end{array}$ & $\begin{array}{c}0.423 \\
(0.449)\end{array}$ & $\begin{array}{c}0.467 \\
(0.441)\end{array}$ & $\begin{array}{c}* * \\
(\mathrm{NS})\end{array}$ \\
\hline SI & 26 & $\begin{array}{l}3.27 \\
(3.30)\end{array}$ & $\begin{array}{c}3.01 \\
(3.01)\end{array}$ & $\begin{array}{c}1 \\
(1)\end{array}$ & $\begin{array}{c}0.183 \\
(0.098)\end{array}$ & $\begin{array}{l}\text { NS } \\
(\mathrm{NS})\end{array}$ & $\begin{array}{c}0.345 \\
(0.367)\end{array}$ & $\begin{array}{c}0.423 \\
(0.407)\end{array}$ & $\begin{array}{l}\mathrm{NS} \\
(\mathrm{NS})\end{array}$ \\
\hline $\mathrm{SC}$ & 13 & $\begin{array}{c}4.46 \\
(4.50)\end{array}$ & $\begin{array}{c}4.45 \\
(4.50)\end{array}$ & $\begin{array}{c}3 \\
(3)\end{array}$ & $\begin{array}{c}0.167 \\
(0.173)\end{array}$ & $\begin{array}{c}* * \\
(\mathrm{NS})\end{array}$ & $\begin{array}{c}0.476 \\
(0.469)\end{array}$ & $\begin{array}{c}0.567 \\
(0.563)\end{array}$ & $\begin{array}{l}\mathrm{NS} \\
(\mathrm{NS})\end{array}$ \\
\hline
\end{tabular}




\section{Population differentiation}

The Kapiti population showed significant genetic differentiation from the WR populations $\left(F_{\mathrm{ST}}\right.$ $=0.11-0.14, P<0.0001)$, as summarized in Table 5.4A. $F_{\mathrm{ST}}$ pairwise comparisons were significantly different between all population pairs $(P<0.0001)$, except for between Breaker Bay and the South Coast population. These two populations were not genetically different $\left(F_{\mathrm{ST}}\right.$ $=0.002, P=0.71$ ). There was an isolation by distance pattern (Mantel test, $\mathrm{r} 2=0.96, P=0.04$, Figure 5.3), and AMOVA (see Table 5.5A) revealed that there was significant differentiation at three levels: (1) among regions (e.g. WR and Kapiti Island, $P<0.0001$ ), (2) among populations (e.g. BB, SI, SC and KP; $P<0.0001$ ), and (3) within populations $(P<0.0001)$. The greatest source of variation was attributable to within population variation (83\%), while the lowest was attributable to among population variation (6\%). DAPC showed separation between the Kapiti and WR populations (Figure 5.4A), and within the WR populations, Breaker Bay overlapped with the South Coast population, but both were distinct from MatiuSomes Island (as also supported by their $F_{\mathrm{ST}}$ values, $F_{\mathrm{ST}}=0.042-0.053, P<0.0001$ ). A STRUCTURE plot showed a similar relationship, where three genetic clusters revealed that the Kapiti Island population exhibited a different genetic structure compared to the WR populations. Within the WR populations, Matiu-Somes Island was genetically different from Breaker Bay and the South Coast, which were genetically similar (Figure 5.4A).

Table 5.4. Population pairwise $F_{\mathrm{ST}}$ values, where bolded values in grey indicate significant differentiation $(P<0.0001)$. Populations are as follows: Breaker Bay excluding clones $(\mathrm{BB}-$ $\mathrm{C})$, Breaker Bay including clones $(\mathrm{BB}+\mathrm{C})$, Kapiti Island (KP), Matiu-Somes Island (SI), and South Coast (SC). Two tables represent analysis excluding clones (A) versus analysis including clones at Breaker Bay (B).

\begin{tabular}{|c|cccc|}
\hline $\mathrm{A}$ & $\mathrm{BB}-\mathrm{C}$ & $\mathrm{KP}$ & $\mathrm{SI}$ & $\mathrm{SC}$ \\
\hline $\mathrm{BB}-\mathrm{C}$ & 0 & & & \\
$\mathrm{KP}$ & $\mathbf{0 . 1 2 0}$ & 0 & & \\
$\mathrm{SI}$ & $\mathbf{0 . 0 4 2}$ & $\mathbf{0 . 1 4 2}$ & 0 & \\
$\mathrm{SC}$ & 0.002 & $\mathbf{0 . 1 1 0}$ & $\mathbf{0 . 0 5 3}$ & 0 \\
\hline
\end{tabular}

\begin{tabular}{|c|cccc|}
\hline $\mathbf{B}$ & $\mathrm{BB}+\mathrm{C}$ & $\mathrm{KP}$ & $\mathrm{SI}$ & $\mathrm{SC}$ \\
\hline $\mathrm{BB}+\mathrm{C}$ & 0 & & & \\
$\mathrm{KP}$ & $\mathbf{0 . 1 2 4}$ & 0 & & \\
$\mathrm{SI}$ & $\mathbf{0 . 0 4 7}$ & $\mathbf{0 . 1 4 2}$ & 0 & \\
$\mathrm{SC}$ & 0.012 & $\mathbf{0 . 1 1 0}$ & $\mathbf{0 . 0 5 3}$ & 0 \\
\hline
\end{tabular}




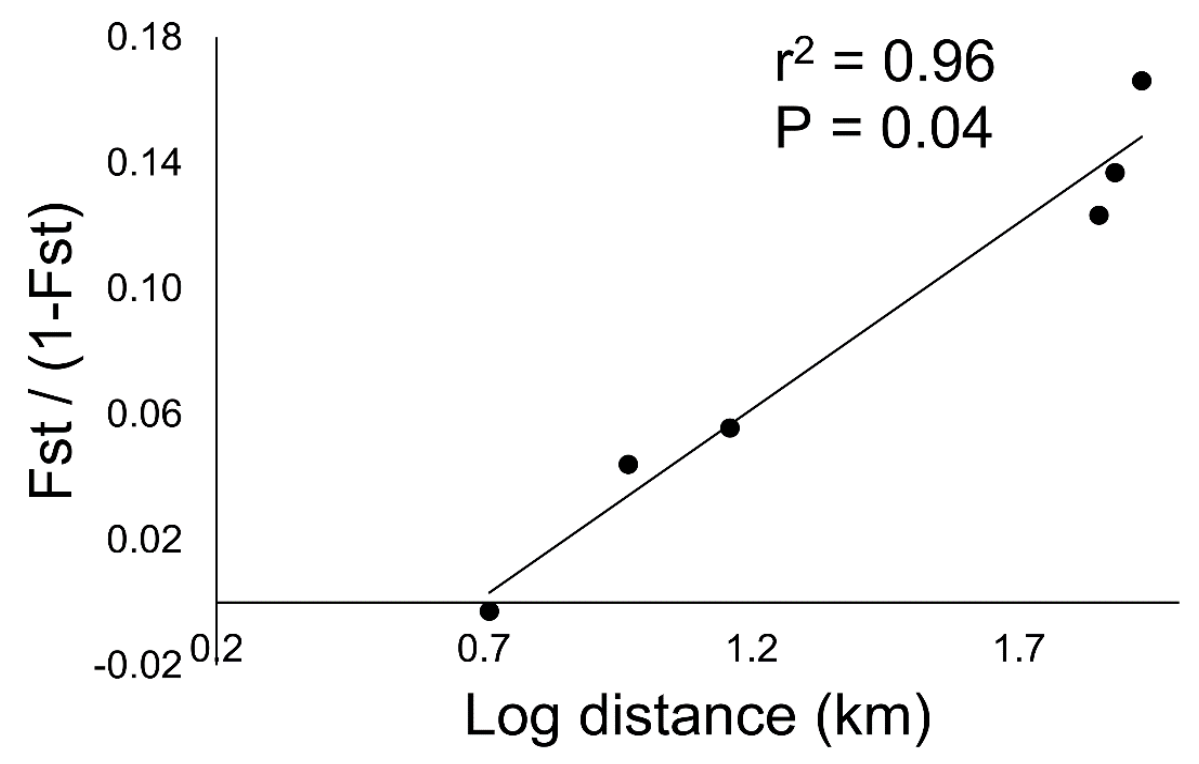

Figure 5.3. Isolation by distance for populations of Tethya burtoni, where pairwise $F_{\mathrm{ST}} /\left(1-F_{\mathrm{ST}}\right)$ between populations is plotted against the logarithm of geographical distances $(\mathrm{km})$ between populations. P-value determined by a Mantel test.

Table 5.5. Results from an analysis of molecular variance (AMOVA), where regions refer to the differences between the Kapiti Island population and the Wellington region (WR) populations. Populations in the WR include: Breaker Bay (BB), Matiu-Somes Island (SI), and the South Coast (SC). Two tables represent analysis excluding clones (A) versus analysis including clones at Breaker Bay (B).

\begin{tabular}{|c|c|c|c|c|c|c|}
\hline $\begin{array}{l}\text { Source of } \\
\text { variation }\end{array}$ & $\begin{array}{l}\text { Degrees } \\
\text { of } \\
\text { freedom }\end{array}$ & $\begin{array}{l}\text { Sum of } \\
\text { squares }\end{array}$ & $\begin{array}{c}\text { Variance } \\
\text { component }\end{array}$ & $\begin{array}{c}\text { Variance } \\
(\%)\end{array}$ & $\begin{array}{l}\text { Fixation } \\
\text { indices }\end{array}$ & P-value \\
\hline \multicolumn{7}{|l|}{$\mathbf{A}$} \\
\hline Among Regions & 1 & 53.488 & 0.872 & $11 \%$ & 0.113 & $<0.0001$ \\
\hline Among Pops & 2 & 30.066 & 0.441 & $6 \%$ & 0.065 & $<0.0001$ \\
\hline Within Pops & 87 & 555.742 & 6.388 & $83 \%$ & 0.170 & $<0.0001$ \\
\hline \multicolumn{7}{|l|}{$\mathbf{B}$} \\
\hline Among Regions & 1 & 56.982 & 0.834 & $11 \%$ & 0.109 & $<0.0001$ \\
\hline Among Pops & 2 & 36.548 & 0.537 & $7 \%$ & 0.079 & $<0.0001$ \\
\hline Within Pops & 97 & 609.490 & 6.283 & $82 \%$ & 0.179 & $<0.0001$ \\
\hline
\end{tabular}



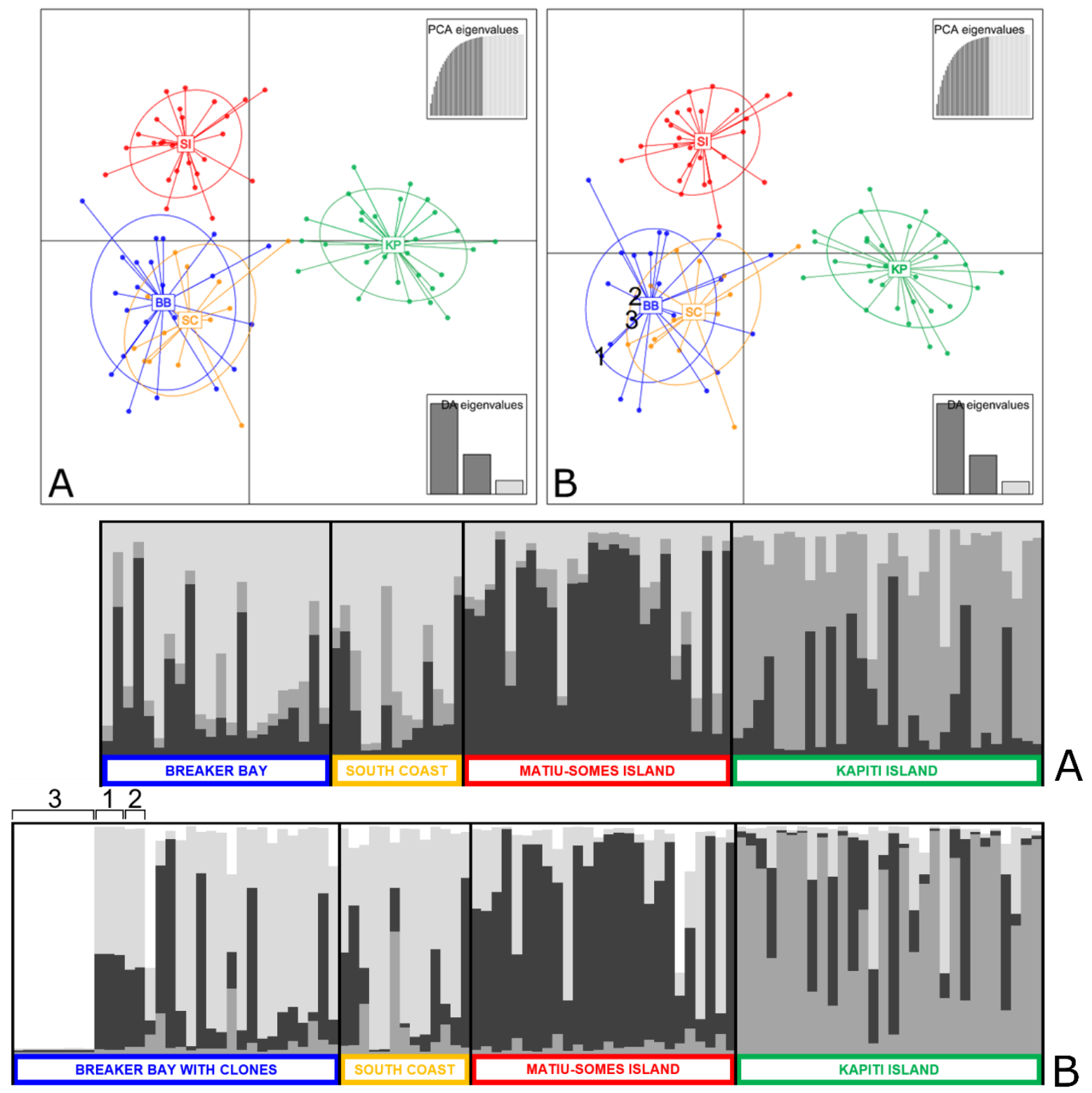

Figure 5.4. Population differentiation between four populations (Breaker Bay, BB; Kapiti Island, KP; South Coast, SC; Matiu-Somes Island, SI) of Tethya burtoni in central New Zealand. Plots labelled 'A' correspond to the analysis run excluding clones, where ' $\mathrm{B}$ ' includes clones for the Breaker Bay population. Top: DAPC based on microsatellite allele frequencies, where each point represents a T. burtoni genotype and populations (BB, KP, SC, SI) are labelled within $67 \%$ of their inertia ellipses. Bottom: STRUCTURE plots, where each vertical line represents a sponge and the coloration corresponds to the estimated percent chance that an individual belongs to each of the genetic clusters $(\mathrm{K}) . \mathrm{K}=3$ for STRUCTURE plot ' $\mathrm{A}$ ' and $\mathrm{K}=4$ for plot ' $\mathrm{B}$ '. Clone groups labelled in 'B' plots (1, 2, 3; see Appendix 7 for more information on clone groups). 


\section{Asexual reproduction}

There were four groups of sponges containing MLGs at Breaker Bay (Figure 5.5). Because the probability of these MLGs being a product of sexual reproduction was almost zero in all instances, these MLGs were considered clones and referred to clones hereafter. Each group was a different genotype and was contained within an area of less than $900 \mathrm{~cm}^{2}$ (see Appendix
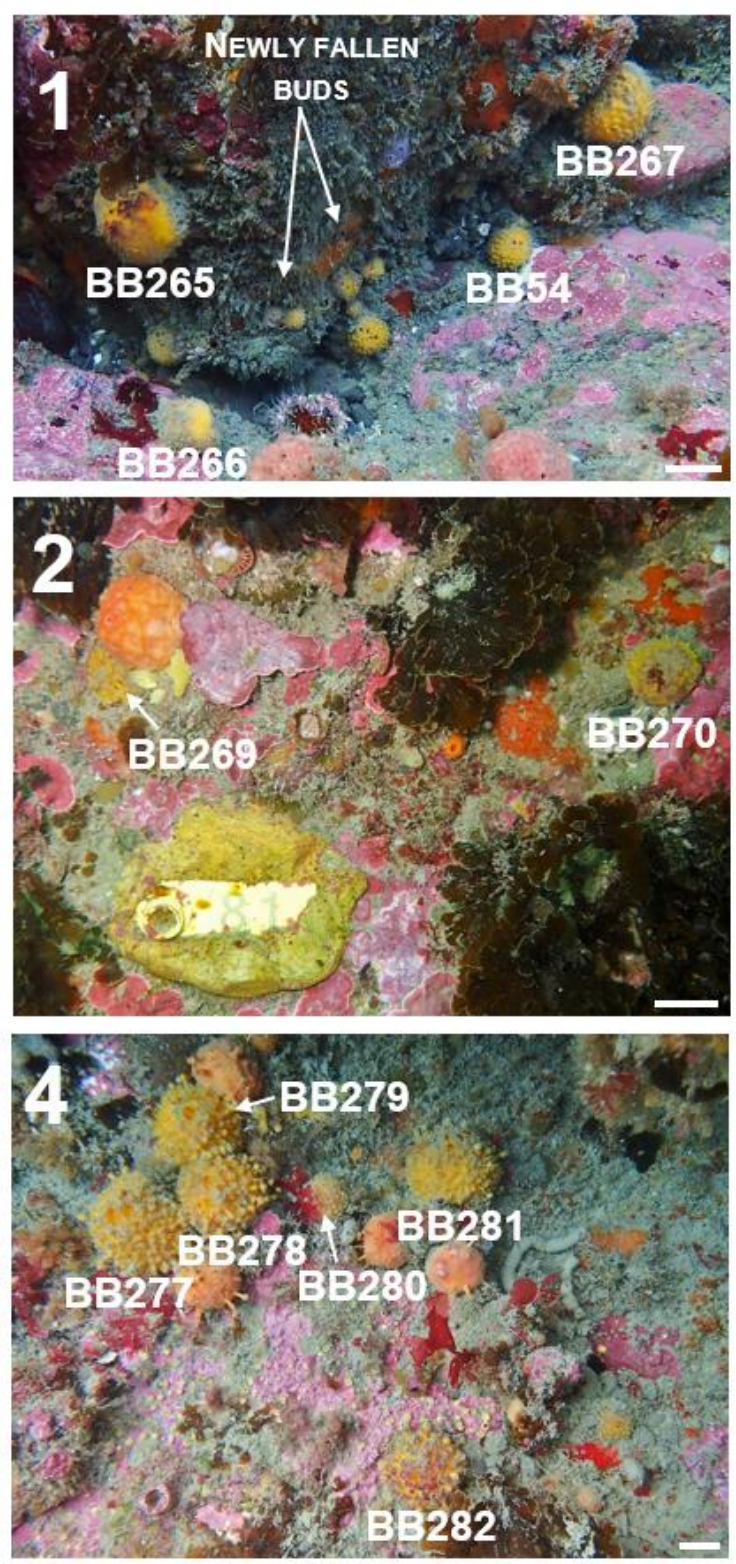
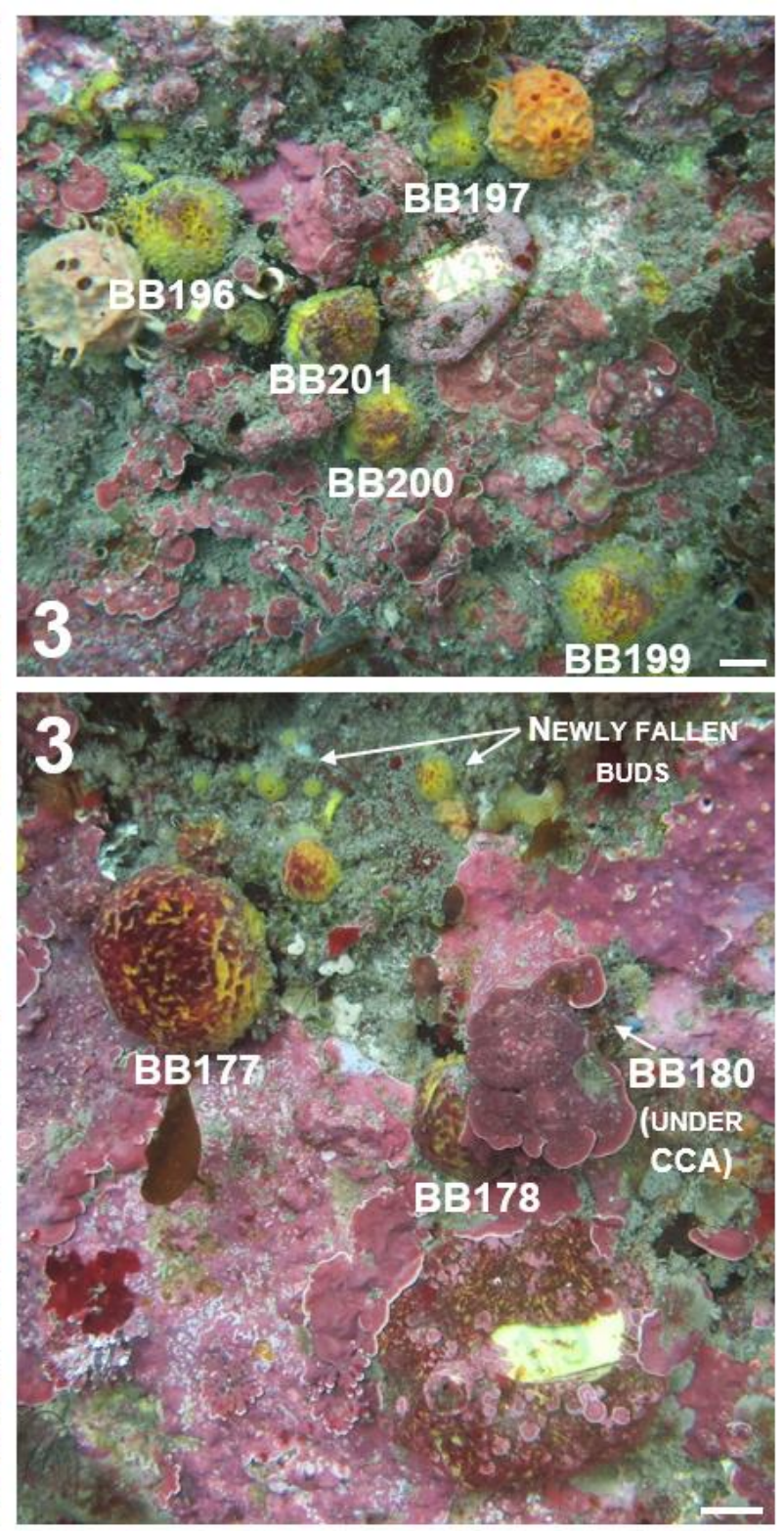

Figure 5.5. Clone groups of Tethya burtoni found at Breaker Bay. Numbers (1-4) and labels (BB\#) correspond to clone group and sponge IDs, respectively (see Appendix 7, Table A7) for more information on clone groups). Sponge IDs written below the sponge that it corresponds to, unless otherwise indicated by an arrow. Newly fallen buds that settled and grew over the monitoring period labelled. White lines in bottom right corner of each picture represent scale bar $=20 \mathrm{~mm}$. Also pictured but not relevant to this study are T. bergquistae, which is an orange or pink golf ball sponge. 
7, Table A7). Over the 2015 - 2017 monitoring period, mortality within the clone groups was $40 \%$. Two of the clone groups exhibited asexual reproduction, where all members of one group (Group 1) budded over the spring period and all members of the other group (Group 4) budded at various times throughout the year (Appendix 7). Three clone groups were still living during summer 2018 and available for tissue collection during their annual sexual reproductive event. One sponge from each clone group contained gametes (either oocytes or spermatic cysts), totalling three sexually reproductive sponges out of 13 individuals (Figure 5.6). For the ten control sponges collected, histological analyses revealed that five sponges contained oocytes, two sponges contained spermatic cysts and three sponges were not reproductive. Fisher's exact test revealed significant differences in the proportion of sexually reproductive individuals between the clone groups and the control group $(P=0.04)$. The size frequency distribution of total collected sponges ranged from $8-37 \mathrm{~mm}$ in diameter, with $80 \%$ of the sizes being $13-$ $26 \mathrm{~mm}$. For the control group, sponges ranged from $12-22 \mathrm{~mm}$ and for the clone groups, sizes ranged from $12-27 \mathrm{~mm}$. The smallest individuals found to contain gametes had a diameter of $12 \mathrm{~mm}$, whereas the largest was $36 \mathrm{~mm}$ (Figure 5.7). All of the clones sampled were within the size range of sexually reproducing sponges. For Group 1, all clones were found with a radial area of $18 \mathrm{~cm}$ from the sponge that contained gametes. For Group 3, all clones were found within $32 \mathrm{~cm}$ and for Group 4, within $39 \mathrm{~cm}$.
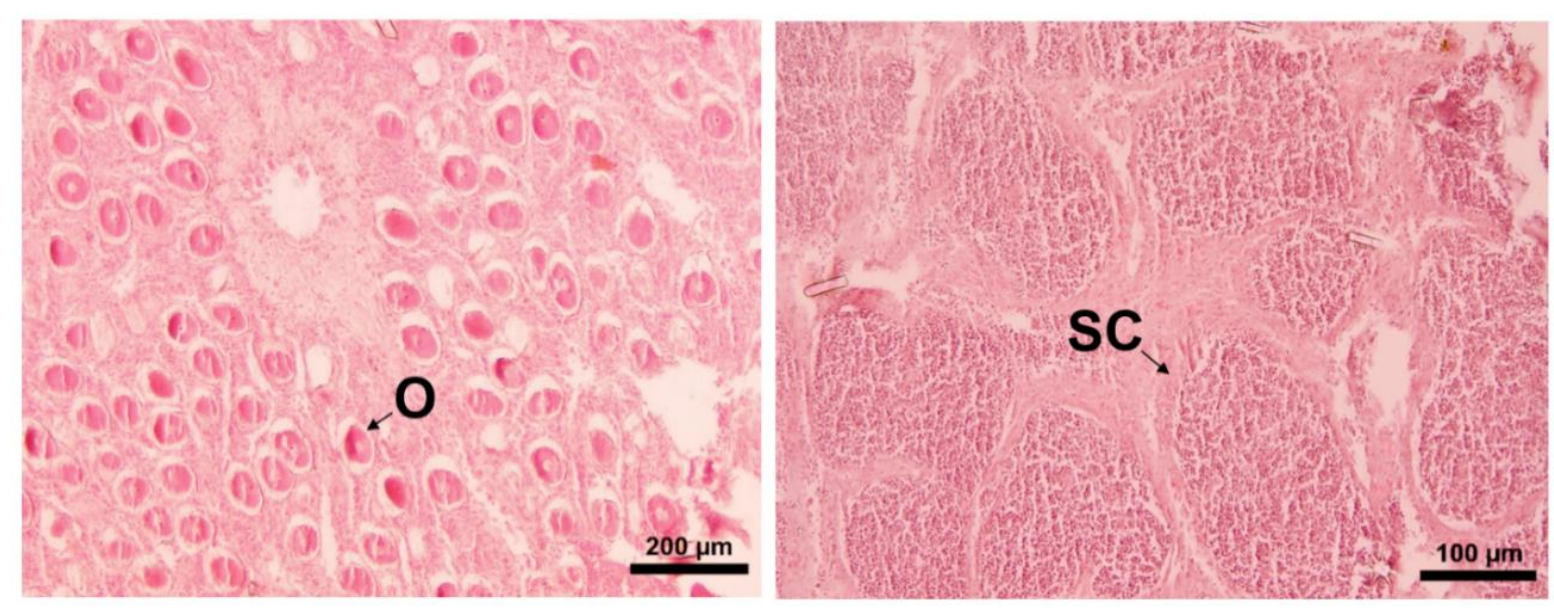

Figure 5.6. Images of sections from sexually reproductive specimens of Tethya burtoni. Left: Female sponge containing oocytes (o). Right: Male sponge containing spermatic cysts (sc). 


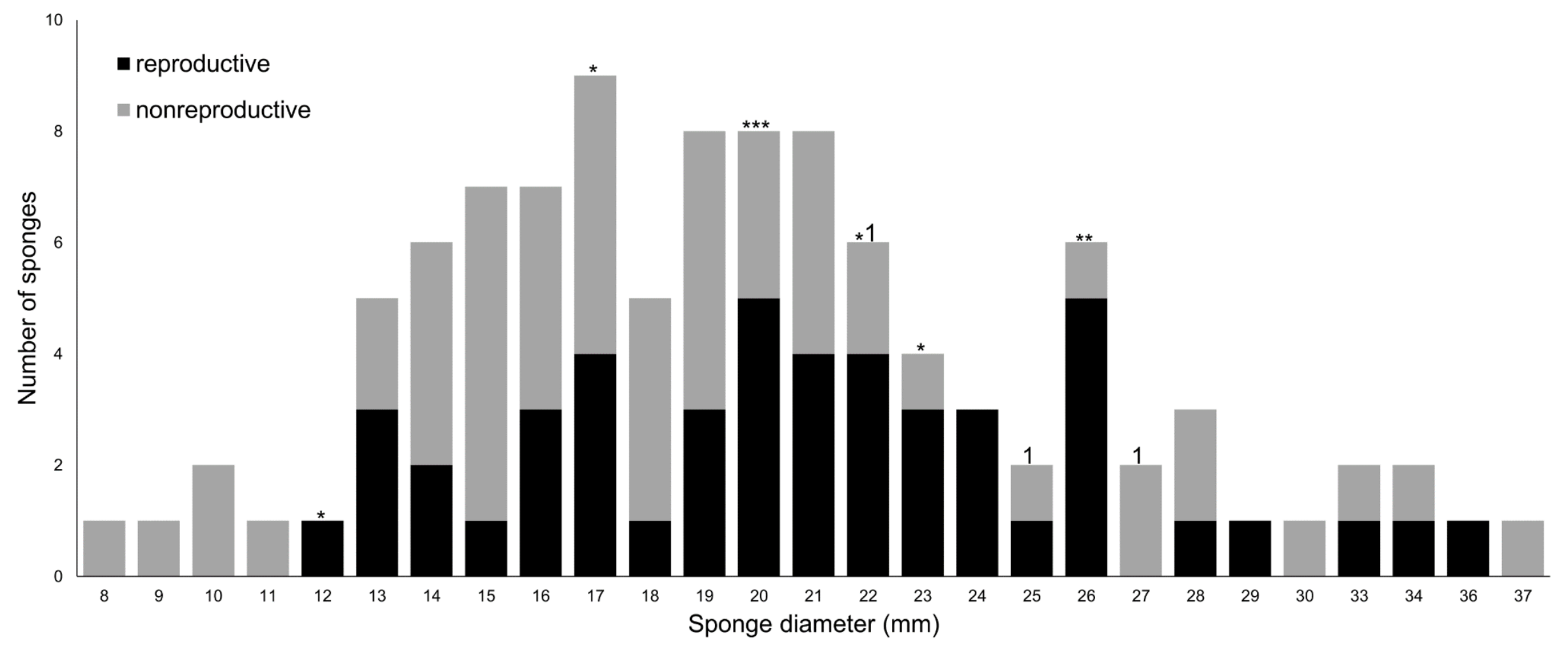

Figure 5.7. Size frequency distribution of Tethya burtoni. Size classes broken by every mm. Grey bars correspond to non-reproductive sponges, and black bars represent reproductive sponges. Each symbol above the bars (either asterisk or number) corresponds to a clone sampled from clone groups, with the sampled clone placed above its size class (see Appendix 7, Table A7 for information on clone groups). An asterisk means that the clone was non-reproductive, whereas a ' 1 ' means that the clone contained gametes (either oocytes or spermatic cysts). 
The genetic diversity indices for the Breaker Bay population including clones were similar to those of Breaker Bay without clones (Table 5.1). One more locus (TBURT9) became out of HWE when clones were included in the analysis. When comparing the population with and without clones (Table 5.2), $F_{\text {IS }}$ decreased from 0.061 to 0.008 . The Breaker Bay population that included clones was out of HWE when using all loci and also when using only those loci that were in HWE. Another difference was that the analysis with clones yielded locus pairs in linkage disequilibrium across all populations (16 pairs), as opposed to the analysis removing the clones (which yielded no pairs of loci exhibiting linkage disequilibrium). Pairs in linkage disequilibrium were only significant in the Breaker Bay population (25 pairs), and not for locus pairs in any other population (Appendix 8, Table A8). Pairwise $F_{\mathrm{ST}}$ values between Breaker Bay with clones and the other three populations slightly increased (by $0.003-0.01$ units, see Table 5.4B) and AMOVA results were similar (Table 5.5B). The DAPC plot revealed the same clustering as the analysis with Breaker Bay without clones (Figure 5.4B). The number of genetic clusters identified by STRUCURE increased from $K=3$ (without clones) to $K=4$ (with clones), with the fourth genetic cluster mainly belonging to one clone group at Breaker Bay (Group 3).

\section{Discussion}

In this chapter, I found differential roles of sexual and asexual reproduction in connectivity and population genetic structure for Tethya burtoni in central New Zealand. Due to both the short pelagic duration of sponge larvae (Maldonado, 1998) and high incidences of asexual budding by $T$. burtoni (M. Shaffer, personal observation), I expected to find a local retention of genotypes and high instance of clones, resulting in the following genetic characteristics: (1) low genetic diversity within populations, (2) linkage disequilibrium, (3) high levels of inbreeding within populations, and (4) strong population structure over small scales. Instead, populations were relatively (to other sponge populations) diverse, displayed no linkage disequilibrium, were not inbred, and were more connected than expected. When examining the fate of the buds and role of clones, buds did not disperse far and clones had reduced instances of sexual reproduction, suggesting that they are less important in gene flow and are instead likely to be more important for genotype persistence. Thus, clonal reproduction does not appear to play a role in large-scale population structure, but patches of clonal organisms structure these populations at the intrapopulational level. 


\section{Connectivity and population structure}

I expected to find highly structured populations across even relatively small scales of $5-10 \mathrm{~km}$, due to the limited pelagic larval duration (PLD) previously reported for sponges (Mariani et al. 2001; Maldonado and Bergquist, 2002; Mariani et al, 2006), and the occurrence of clones in T. burtoni, which would be expected to amplify local genotypes. Recruitment of local genotypes along with clonal propagation was expected to result in highly differentiated populations over small spatial scales. While Kapiti Island was significantly genetically different from the WR populations $\left(F_{\mathrm{ST}}=0.11-0.14, P<0.0001\right)$, there was more connectivity within the WR populations than expected. For example, the Breaker Bay and South Coast populations were genetically similar, suggesting high levels of connectivity. The dynamic environment of the coast of Wellington, along with the short PLD of the oviparous T. burtoni, likely allows these two populations to be connected over distances of $5 \mathrm{~km}$. While the PLD of T. burtoni is currently unknown, larvae (zygotes) appear be in the water column long enough to allow populations over $5-10 \mathrm{~km}$ to be connected. Matiu-Somes Island was significantly genetically differentiated from the Breaker Bay and South Coast populations $\left(F_{\mathrm{ST}}=0.04-\right.$ $0.05, P<0.0001)$. This site is in Wellington Harbour, which is sheltered and has a long flushing time (10 days; Heath, 1974). This fact may give an indication that the PLD of T. burtoni is shorter than 10 days, and therefore T. burtoni larvae are not pelagic long enough to enter the harbour suspended in the water column, which could explain the restricted gene flow observed between the harbour and non-harbour sites. These results are generally consistent with other sponge connectivity studies (Chaves-Fonnegra et al., 2015; Blanquer and Uriz, 2010; Riesgo et $a l ., 2016)$, showing limited gene flow across larger distances (i.e., over $50 \mathrm{~km}$ ); however, there is currently a lack of studies examining population structure at a smaller scale $(5-10 \mathrm{~km})$, as employed in this study, and so comparisons are hard to draw.

Because of the high incidence of asexual reproduction, I hypothesized that a larger number of clones would have been collected, even when sampling 3-5 m apart. This large number of clones, coupled with limited larval duration of sponges, would be expected to promote local recruitment and result in low genetic diversity (Procaccini and Mazella, 1998), linkage disequilibrium (Charlesworth and Wright, 2001; Black and Johnson, 1979; Ayre and Hughes, 2000; Duran et al., 2004b), inbreeding (Li and Ge, 2001; Ayre, 1984), and strong genetic structure. Despite these common characteristics for such sessile asexual populations, the 
opposite for these populations of $T$. burtoni was found. No instances of clones from populations where I collected $3-5 \mathrm{~m}$ apart were discovered, despite the high frequency of budding events observed for T. burtoni. Duran et al. (2004b) suggested that the incidence of clones may not correlate with the frequency of asexual reproductive events. These authors sampled individuals of Crambe crambe $5 \mathrm{~m}$ apart to avoid collecting clones, the same sampling regime employed for this study, but despite this, the authors found clones. Because $C$. crambe was found to have low instances of asexual reproduction (Turon et al., 1998), the high instance of clones was unexpected and called for a revaluation of the role of asexual reproduction in the structure of this population. Patches of clonal organisms in a small spatial scale have been found for other organisms, including seagrass and corals (Baums et al, 2006; Alberto et al., 2005; Arnaud-Haond et al., 2006; Arnaud-Haond et al., 2007), where clonal patches are thought to play a role in seeding the population (Alberto et al., 2005), local adaptation (Arnaud-Haond et al., 2006), or recovery from damage (Baums et al., 2006). Because no clones within populations of T. burtoni were collected when sampling 3-5 m apart, no clones were included in the main analysis at the interpopulational scale. Instead, clonal organisms had an influence on a small, intrapopulational scale. There was no evidence of low genetic diversity for these populations of T. burtoni, as our heterozygosity measures were similar to those reported for other sponge populations (e.g., Duran et al., 2004b; Blanquer et al., 2009; Dailianis et al. 2011; Riesgo et al., 2016). Furthermore, there were no signs of linkage disequilibrium nor significant inbreeding across populations. While there was structure across populations, some populations were more connected than expected across the sampled scale. An AMOVA revealed that most variation was due to within population variability compared to between populations/regions, which is more consistent with populations that are panmictic, rather than asexually reproducing populations or populations having restricted connectivity. Overall, it appears that asexual reproduction is not important for connectivity, and that larval dispersal occurs over greater distances for our study species than originally expected, allowing a greater level of connectivity and lower levels of inbreeding and linkage disequilibrium.

The idea that asexual reproduction does not contribute to the interpopulational scale is further exemplified when running the population genetic analyses with and without clones. For both analyses, the overall heterozygosity and population structure remained the same. The main differences between the genetic structure of the Breaker Bay population with and without 
clones were that the former was out of HWE and contained linked alleles (as expected, as all genes are inherited together during asexual reproduction). In a similar study for sponges belonging to the genus Chondrilla, linkage disequilibrium only occurred when genets (i.e., clones) were included in the analysis (Zilberberg et al., 2006), providing further evidence that asexual reproduction results in the occurrence of linked alleles. The STRUCTURE analysis showed that Breaker Bay was more genetically patchy, due to the clonal groups of T. burtoni. Had clones been included for analyses for all four populations (i.e., collection without sample restrictions), it is likely that all locations would contain patches of clonal organisms. Therefore, clonal organisms in this population are relevant when examining the intrapopulational level. At this level, clones may important for population maintenance, genotype longevity, and/or size regulation of individuals.

\section{Population structure in a New Zealand context}

This study is the first that examines genetic population structure for sponges in New Zealand. Similar to the findings for other invertebrates in New Zealand (see Ross et al. (2009) and Gardner et al., (2010)), an isolation by distance pattern was observed for T. burtoni. Throughout New Zealand, a negative relationship between PLD and genetic differentiation has been observed across multiple studies (Ross et al., 2009) The PLD for T. burtoni is currently unknown, so it is difficult to draw conclusions as to whether the isolation by distance pattern observed here is related to a short PLD. For other sponges, PLDs have been found to range from minutes (Uriz et al., 1998) to hours (Bergquist and Sinclair, 1968) to days (Ayling, 1980). There are only a few instances of PLD lasting longer, from weeks to months, and this rarity is often achieved through adaptive mechanisms. For instance, species of Alectona have adapted planktonic armoured propagules (Vacelet, 1999), which likely allows them to remain as planktonic larvae for longer periods of time (i.e., months; Maldonado, 2006). Because longer PLDs for sponges is rarer, it is instead a likely possibility that the PLD of T. burtoni is relatively short, which contributes to the isolation by distance pattern of differentiation observed here.

Many population structure studies conducted in New Zealand reveal a north-south differentiation, where the observed restricted gene flow is generally attributed to upwelling or the movement of currents between the North and South Islands (Ross et al., 2009; Gardner et $a l ., 2010)$. Many studies indicate that barriers to gene flow exist in central New Zealand, near 
the Cook Strait region (e.g., Apte and Gardner 2002, Veale 2007). This region has multiple currents (the D'Urville, Southland and East Cape Currents) that interact (Heath, 1971), which may either promote or inhibit the mixing of larvae. For phylogenetic studies conducted in this Cook Strait region, there are often genetically distinct individuals that belong to both northern and southern populations living in sympatry. For instance, Veale and Lavery (2011) found a strong north-south division for chitons, but in the Cook Strait region, found a mix of haplotypes from the north and south. Connectivity, however, is studied to a lesser extent in this region. For $T$. burtoni, differentiation between the Wellington region and Kapiti Island was evident, which may be attributable to PLD, but also can be due to hydrological barriers. For instance, the D'Urville Current transports water from northwest to southeast through the Cook Strait, and organisms in Wellington may not be able to travel northward towards Kapiti. Further, the PLD of T. burtoni may inhibit gene flow from Kapiti to the Wellington region.

\section{Role of asexual reproduction}

To better resolve the role of asexual reproduction, I investigated the fate of the buds and the role of clones at the intrapopulation level. The dispersal of buds was found to occur within less than a square meter, which further confirms that buds do not play a role in gene flow by connecting individuals to other populations. The lack of dispersal of clonal offspring has been observed for other asexual organisms, where clones have been recorded to have a more limited dispersal than larvae (Jackson and Coates, 1986; McFadden, 1997). If clones were restricted in their dispersal, patchy populations over small spatial scales would emerge, as quick amplification of certain genotypes through asexual reproduction would cause populations to diverge. However, panmixis between close populations was observed and further, clones did not appear to significantly alter the genetic structure of the population. Therefore, the reproductive potential of the clones was examined to better understand how clonal organisms contributed to the gene pool. For each clone group sampled, only one individual contained gametes. While new clones may be smaller and therefore unable to reproduce, all of the clones sampled belonged to size classes that have been previously found to contain sexually reproductive individuals. Therefore, it could be that for each clone group, the observed gametes only occurred in the parent sponge (since only one clone was sexually reproducing) and that the rest of the clonal offspring were sterile. This hypothesis needs further investigation as I was unable to identify the parent sponge that produced the offspring, or the generation of any of the 
clonal sponges. With such a small dispersal range, it would appear evolutionarily disadvantageous for a small patch of clones to release gametes because such self-fertilization would lead to higher rates of inbreeding (Jackson and Coats, 1986). My sample size was low due to the lack of clones tagged, coupled with a high mortality rate of clones (40\%) over two years, and therefore this hypothesis warrants further investigation where parental relationships are tracked. Nonetheless, sexual reproduction in clonal groups versus the control group was significantly lower, suggesting that clones have reduced reproductive ability and do not contribute to the gene pool in the same manner. Wulff (1991) found that by asexually reproducing, branching sponges increased and maintained a genotype within a population. For T. burtoni, while increased clones would secure a specific genotype and keep population density high, the lack of ability to sexually reproduce coupled with low dispersal may inhibit the chance of that genotype remaining in the population through sexual reproduction. Genotypes could also be maintained through continued asexual reproduction, and instances of budding were viewed in these clone groups. Muller's ratchet (Muller, 1932; Gabriel et al., 1993), however, would be expected to make an allele deleterious through the accumulation of mutations. This would result in the removal of that gene from the population overtime, lessening the chance for alleles passed on asexually to persist over time. Furthermore, the continued persistence of asexual reproduction would likely cause clones to disperse further and be found more frequently in the population, which was not evident for T. burtoni.

Asexual reproduction instead may be important for individual survivorship, which may have consequences for long term population maintenance. Asexual reproduction has been shown to be important for maintaining populations of Hymediacidon perlevis. For this sponge, increased fragmentation (and the associated reduced surface area) over winter periods allow sponges to survive in seasonally nutrient depleted waters, and allow the recolonization of the population in subsequent seasons (Gaino et al., 2010). The Wellington south coast waters are characterized by low levels of nutrients (Bowman et al., 1983). Perhaps budding is a size regulation mechanism that prevents $T$. burtoni from becoming too large and needing too many resources that would be unavailable in the surrounding waters. Fusion events, in which clones fuse together, and sometimes with the parental sponge, are common throughout the Tethya genus (e.g., approximately $10 \%$ of population undergoing fusion/year, see Chapter 4). Small buds may remain close to the parent to serve as essentially small energy stores, fusing back to the parent sponge with nutrients/supplies that may be needed for parental survivorship. Size, 
however, was not correlated with budding events for T. burtoni (see Chapter 4), and therefore the exact role asexual reproduction plays remains unclear. Occupation of surrounding space by clones could reduce competition between the parental sponge with other organisms, thereby increasing the chance of its genotype surviving. In this way, asexual reproduction could aid in individual survivorship and promote overall population persistence.

\section{Conclusion}

This chapter highlights the differential roles of sexual and asexual reproduction in population dynamics for T. burtoni in central New Zealand. There was little evidence of local recruitment of individuals within populations, as populations did not display inbreeding or linkage disequilibrium, and instead had a comparatively high diversity. Furthermore, populations were connected over 5-10 km, indicating the PLD is long enough for these sponges to connect across distances of similar scales. High instances of asexual reproduction resulted in patches of clonal organisms within populations, but no clones were found between populations. Thus asexual reproduction appears to play an important role at the intrapopulational level, perhaps aiding in individual sponge survivorship and promoting population maintenance and longevity. Differential roles of these modes of reproduction may have consequences for larger scale population structure and speciation, and understanding their key functions is important for understanding evolutionary history of such organisms. 
CHAPTER 6:

GENERAL DISCUSSION 



\section{Summary of major findings}

This $\mathrm{PhD}$ thesis examines evolutionary processes in both Tethya bergquistae and T. burtoni on temperature reefs in central New Zealand, with the specific aims of delineating species boundaries; characterizing reproductive events and their potential environmental drivers; and determining connectivity patterns within and among populations. With predicted shifts in the climate, this thesis has important implications for how changing environments may affect diversity, reproductive timing and genetic structure of marine populations. Many sessile marine invertebrates share life-history traits with sponges, including complex ways of reproducing, and therefore my results may aid in the understanding of the evolution of other marine taxa. The major findings of this thesis were as follows. In Chapter 2, I found discordance between molecular and morphological methods for species identification for Tethya spp., with likely cryptic speciation within T. burtoni. In Chapter 3, I reported that sexual reproduction is seasonal and occurs from January to March during periods of warm temperature and decreased rainfall, where differences in sexual reproduction between both sites and species existed. In Chapter 4, I found that asexual reproduction occurs continuously throughout the year, with peaks during the austral spring and summer periods. Furthermore, while in situ collections and observations revealed that asexual budding events were correlated with increasing temperature, experimental observations instead revealed that high temperatures did not result in budding (perhaps due to stress). Lack of asexual reproduction in the control treatments, however, may be indicative that asexual reproduction does not occur when temperatures are stable, indicating heterogeneous environments may favour asexual reproduction. In Chapter 5, I discovered that population structure for $T$. burtoni was not reminiscent of highly asexually reproducing populations. Instead, sexual reproduction likely drives drive connectivity patterns, while asexual reproduction is important for genotype survivorship and population maintenance.

In this chapter, I discuss my results in a wider context and first compare methods for species identification based on reproductive ecology, morphology and genetic relationships. I then consider evolutionary forces that shape species boundaries and connectivity patterns, particularly in regard to what conditions may favour both cryptic speciation and introgressive hybridization. I also make comparisons between reproduction in these Tethya spp. to other temperate Australasian sponges to examine species-specific responses, and further discuss species-specific responses observed during the experiment conducted in Chapter 4. Lastly, I 
discuss these results with respect to environmental change and discuss how populations may be altered by climate change.

\section{Comparing methods for species delineations}

The research in this $\mathrm{PhD}$ thesis allowed the delineation of Tethya spp. using an integrated approach based on three metrics: the reproductive biology (both sexual and asexual) of these sponges, morphological differences and genetic evolutionary relationships. Each method revealed a slightly different delineation. For instance, the reproductive biology of Tethya spp. did not allow a confident separation between species, but morphology better allowed the division between $T$. bergquistae and $T$. burtoni. Lastly, genetic analyses revealed the potential for three species of Tethya, with cryptic speciation likely occurring within T. burtoni. Such conflicting species delineations highlight the complexity in defining species and further emphasize the need to use more than one method in order to gain a better understanding of species' boundaries.

\section{Reproductive isolation}

Organisms are often considered different species on the basis of reproductive isolation (Mayr, 1942), which can occur when species have different spawning times, gamete incompatibility, different environmental tolerances/habitat preferences, or conflicting mate preferences (Palumbi, 1994). For closely related species, reproductive isolation often becomes apparent when examining their reproductive ecology, as demonstrated through the delineation of Clathrina spp., where differences in reproductive timing confirmed the division between Clathrina coriacea and C. blanca (Johnson, 1978). Similarly, three sympatric species of the coral Monastraea were confirmed when experimental manipulation revealed failed fertilization between different species and further, differences of in situ spawning times were recorded (Knowlton et al., 1997). In this thesis, the reproductive timing for sexual reproduction in $T$. bergquistae and T. burtoni was generally the same and barriers to reproduction were not obvious. I unfortunately was only able to capture one sexual reproductive event (along with two other partial events), which made inferences about reproductive timing difficult. For the one complete event captured, there appeared to be a difference of a month between the reproductive period for $T$. bergquistae and $T$. burtoni, though this was not reflected in the other 
two partial events captured. This could indicate, however, that there is asynchrony in spawning and fertilization events between both species. The lack of males found during the $2015-2017$ sampling period (Chapters 3) indicate that spermatogenesis is rapid for these sponges. Only one male (T. burtoni) was found during the study period in February 2017 and in the following year in March 2018, three T. burtoni males were found (see Chapter 5), which suggests that spermatogenesis likely occurs sometime between February and March for T. burtoni. More frequent sampling of both $T$. bergquistae and $T$. burtoni during this period may be able to reveal asynchrony between spermatogenesis and perhaps spawning events, which may help identify clearer reproductive differences. Nonetheless, oocyte density and the reproductive output index (ROI) were significantly different between species, suggesting that the species may have different reproductive strategies. Members of the T. burtoni complex (see Chapter 2) were unfortunately unable to be identified based on morphology during analyses relating to sexual reproduction, and without genetic data, differences in sexual reproduction that may occur within this group were not able to be identified.

Species boundaries within Tethya can also be identical on the basis of asexual reproduction, where some species are differentiated from others based on location, frequency and morphology of buds (Bergquist and Kelly-Borges, 1991; Corriero et al., 1996; Sarà and Sarà, 2004; Gaino et al., 2006). While again, differences between species were not necessarily obvious, there was some evidence of separation on the basis of asexual reproduction. For instance, $T$. bergquistae had a higher proportion of budding individuals than T. burtoni, but only at one of the study sites. Furthermore, sponge size had a positive effect on budding events for $T$. bergquistae but not for $T$. burtoni, suggesting that budding may be a size regulation mechanism for T. bergquistae, but may not serve the same purpose for T. burtoni. Furthermore, chlorophyll- $a$ concentration had opposing effects on each species, which may indicate that each species has different drivers of asexual reproduction. Genotype data available for sponges monitored in situ allowed comparisons between members of the T. burtoni complex. While budding events were not significantly different between members of this group, there was a trend in which one group did not bud as much. While there was some indication of differences between species, asexual reproduction appeared to be heavily influenced by the environment, which was further exemplified in the unexpected results obtained from the exploratory experiment examining budding events and temperature in Chapter 4. It was difficult to determine if differences were attributable to interspecific adaptive strategies or rather to 
intraspecific plasticity to environmental conditions, and therefore species delineations based on asexual reproduction cannot be made with certainty.

\section{Morphology}

I assessed morphological differences between $T$. bergquistae and members belonging to the $T$. burtoni complex that were identified using genetic markers. Distinguishing phenotypic plasticity from cryptic diversity remains challenging using comparative morphology (e.g., Vrijenhoek, 2009), especially for species like sponges that have few descriptive features (Hill and Hill, 2002; Xavier et al., 2010). This is important as comparative morphology is the traditional method of species delineation for sponges, and for Tethya in particular, a very extensive taxonomic history exists describing members of this genus. The Tethya genus is particularly diverse in New Zealand, but it can be difficult to identify species from available taxonomic descriptions. A high degree of phenotypic plasticity within New Zealand Tethya suggests that making species delineation based on morphology may be unreliable. For instance, Figure 6.1 shows various images of T. burtoni found in the literature (Pritchard et al., 1984; Sarà and Sarà, 2004) and local identification guides (Battershill et al., 2010; Kelly and Herr, 2015), and allows comparison to specimens of T. burtoni identified in my study (Figure $6.2 \mathrm{~A}, \mathrm{~B})$. Colour proved to be a misleading tool for identification, as T. bergquistae is only described as pink-rose in colour, though on the Wellington south coast, many different colours from dark pink to orange exist (i.e., Figure 6.2D). I also identified sponges that were texturally (and genetically) the same as T. bergquistae, but yellow. While macromorphological differences exist to delineate $T$. bergquistae from $T$. burtoni, I was unable to distinguish members of the $T$. burtoni complex apart, even based on spicule composition. The genetic methods I used consistently revealed more diversity within the T. burtoni group (discussed next), where cryptic speciation in this group is highly likely. Using morphology-based taxonomic techniques for organisms that have few defining features, like sponges, may be a good first step in species identification, but without other taxonomic methods, could lead to conservative estimates of diversity and fail to reveal true divisions of species. 


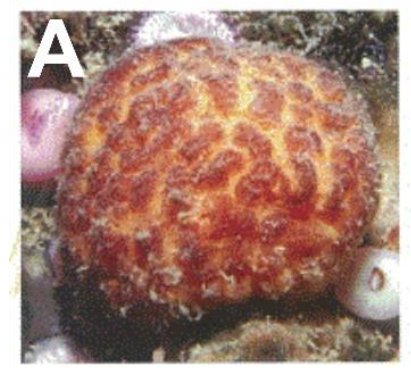

Tethya burtoni. Bottom: Individual sprouting reproductive buds.

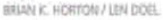
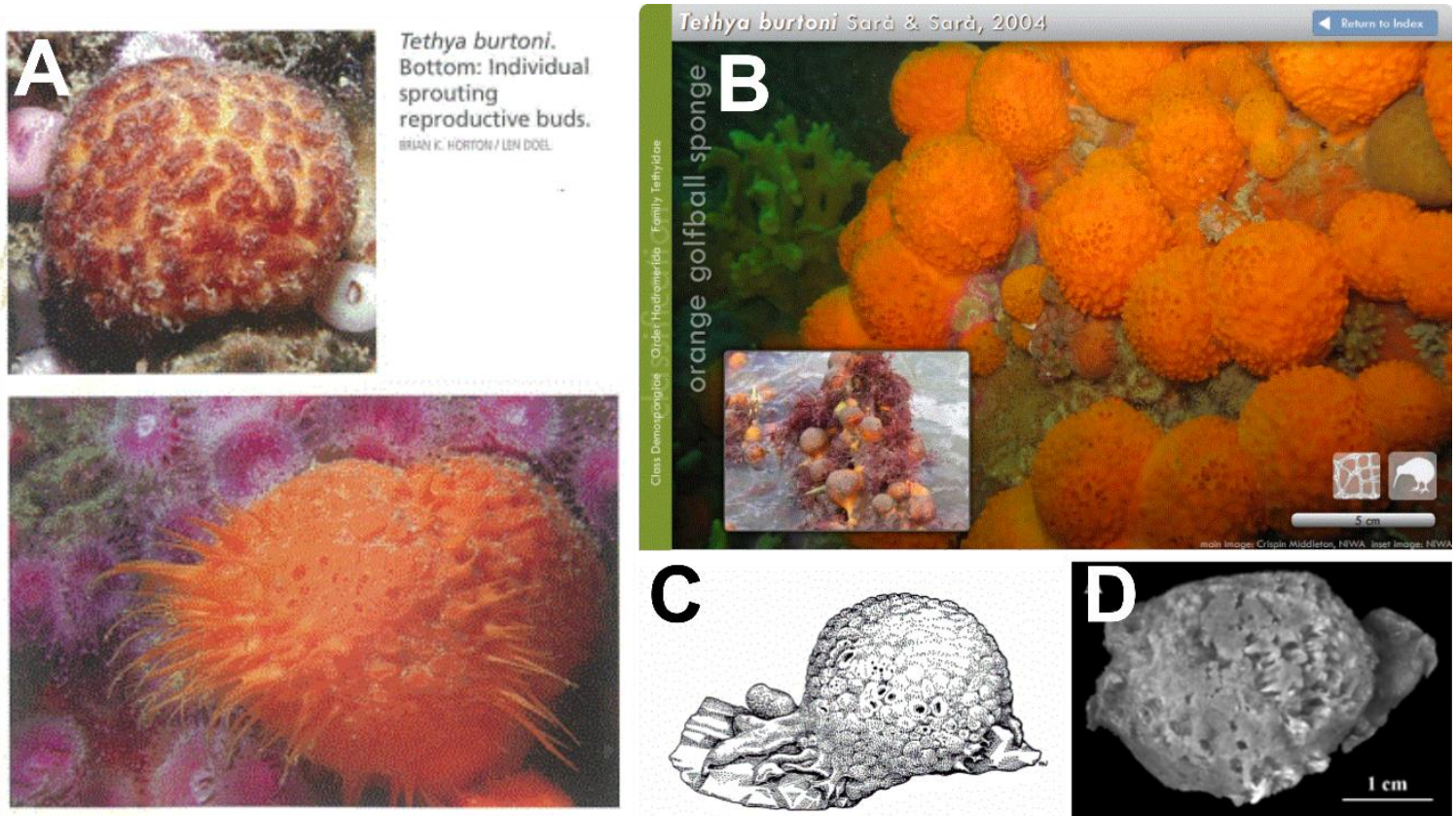

Figure 6.1. Examples of images of Tethya burtoni found in the literature and guidebooks. Citations: A = Battershill et al. (2010); B = from NIWA's identification guide by Kelly and Herr (2015); C = Pritchard et al. (1984); D = an image of the original description of $T$. burtoni in Sarà and Sarà (2004).
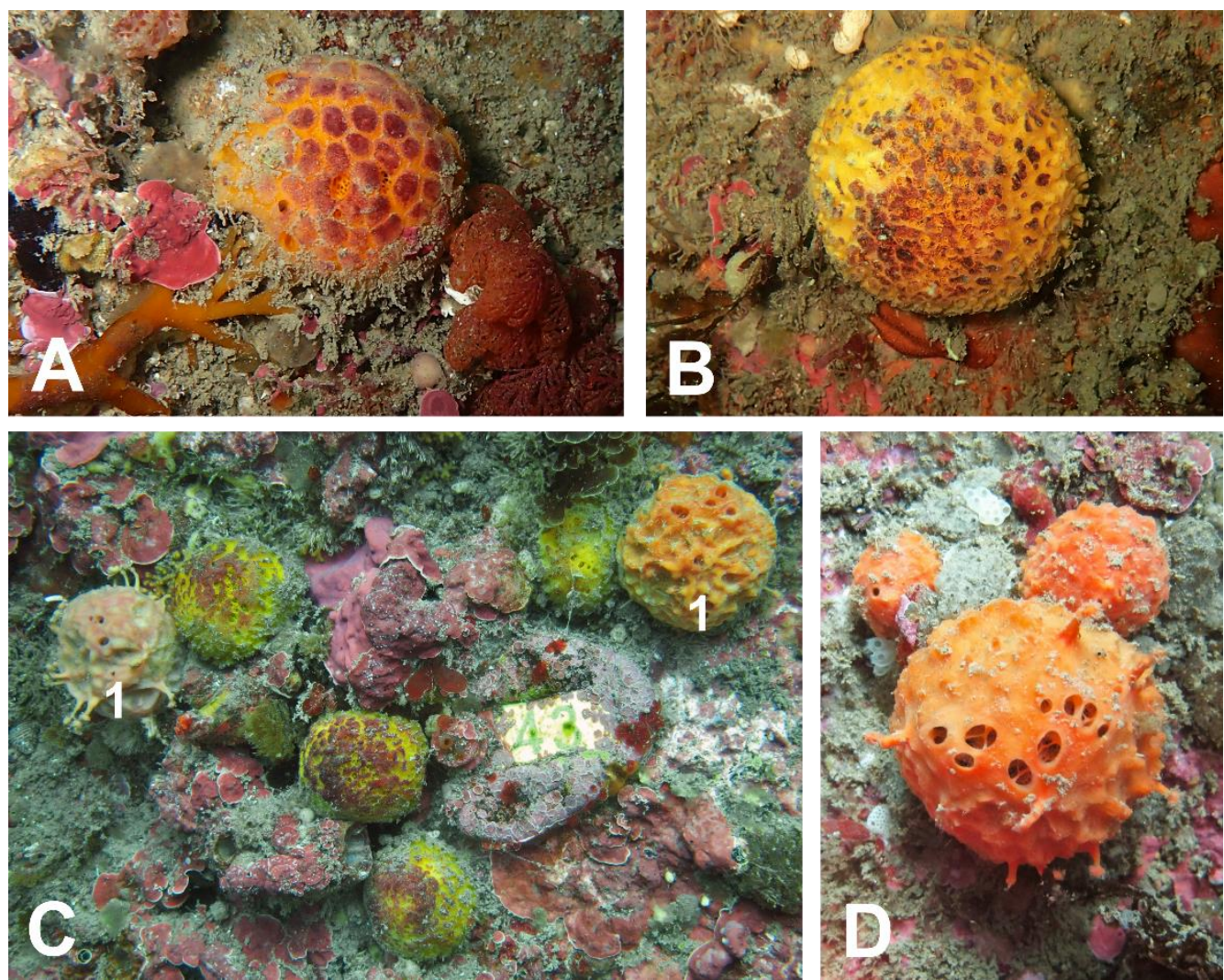

Figure 6.2. Examples of images of Tethya burtoni and T. bergquistae observed in this study. Sponge in A \& B are T. burtoni, while sponges in D are T. bergquistae (based on genetic analyses in Chapter 2). Figure $\mathrm{C}$ shows a group of both $T$. bergquistae and T. burtoni, where $T$. bergquistae are identified using the number 1 and the rest (unlabelled) are T. burtoni. 


\section{Phylogenetic relationships}

The increased use of genetic tools for taxonomy has revealed many new cryptic species (Hebert et al. 2004; Bickford et al., 2007). DNA barcoding in particular has become a recent trend, with the goal of gaining genetic data to characterize species worldwide and to develop a universally consistent species concept (Valentini et al., 2009; Krishnamurthy and Francis, 2012). Despite the accessibility and increased data generated from such tools, there remains controversy surrounding the validity of genetics as a tool for species delineations (Moritz and Cicero, 2004; Meyer and Paulay, 2005; DeSalle et al., 2005), especially when mismatches arise between morphological and genetic data. A challenge in this field includes choosing appropriate markers to use and determining thresholds of divergence that constitute interspecific variation (as opposed to intraspecific diversity). For this study, I used two mitochondrial markers (COI-ext and rnl) and one nuclear marker (18S) to determine the genetic relationship between Tethya spp. All markers revealed a division between T. bergquistae and T. burtoni, and also revealed a division within T. burtoni. Perhaps the strongest evidence for cryptic speciation within T. burtoni is that $18 \mathrm{~S}$, which is a slow evolving marker (Anne, 2006; Stenøien, 2008), revealed two groups within what was previously called T. burtoni. This division was further supported with rnl. And while COI-ext did not reveal the same division within T. burtoni as the other two markers (perhaps due to introgression, though the use of diploid markers would be needed to confirm this), it still revealed a higher degree of diversity within the species than previously thought.

The microsatellite markers developed in Chapter 5 for $T$. burtoni supported the $18 \mathrm{~S}$ and $\mathrm{rnl}$ data. While microsatellite markers are traditionally not used as a tool for phylogenetic studies (but instead population structure analysis), when used here in combination with the other genetic markers gave further evidence for strong genetic differentiation between the T. burtoni clades. The microsatellite markers failed to amplify for T. bergquistae. For T. burtoni, all 11 markers amplified consistently for one of the genetic clades within T. burtoni; but for the other group, some markers failed to amplify, even after reextracting DNA two or three times and rerunning PCR at more relaxed conditions. To measure differentiation between groups, I calculated $F_{\mathrm{ST}}$ using those loci that worked consistently between two groups and found significant and strong differentiation between the $T$. burtoni groups $\left(F_{\mathrm{ST}}=0.39, P<0.0001\right)$. I also assessed differentiation based on clustering methods and for these, considered missing 
information informative. A reconstructed discriminant analysis of principal coordinates (DAPC) including both species clearly shows two genetic groups within T. burtoni (Figure 6.3). In this DAPC, those sponges contained to the left of the DAPC (e.g., populations KP-1, SI-1, BB-1, SC-1) are distinctly separated from the group on the right (e.g., KP-2, BB-2, SC2). These two groups are consistent with the groups produced from the $18 \mathrm{~S}$ and rnl phylograms. The division among these groups clearly show that there is divergence among sponges existing in the same location. In particular, Breaker Bay (BB-1 and BB-2) and the South Coast (SC-1 and SC-2) populations contain sponges that belong to both species. This gives a good indication that divergence within $T$. burtoni is not likely a product of population structure, but instead gives evidence for cryptic speciation and reproductive isolation for sponges living in sympatry. Overall, evidence from multiple molecular markers (conserved regions COI-ext, rnl, 18S; and more variable microsatellite markers) strongly support cryptic speciation within this group.

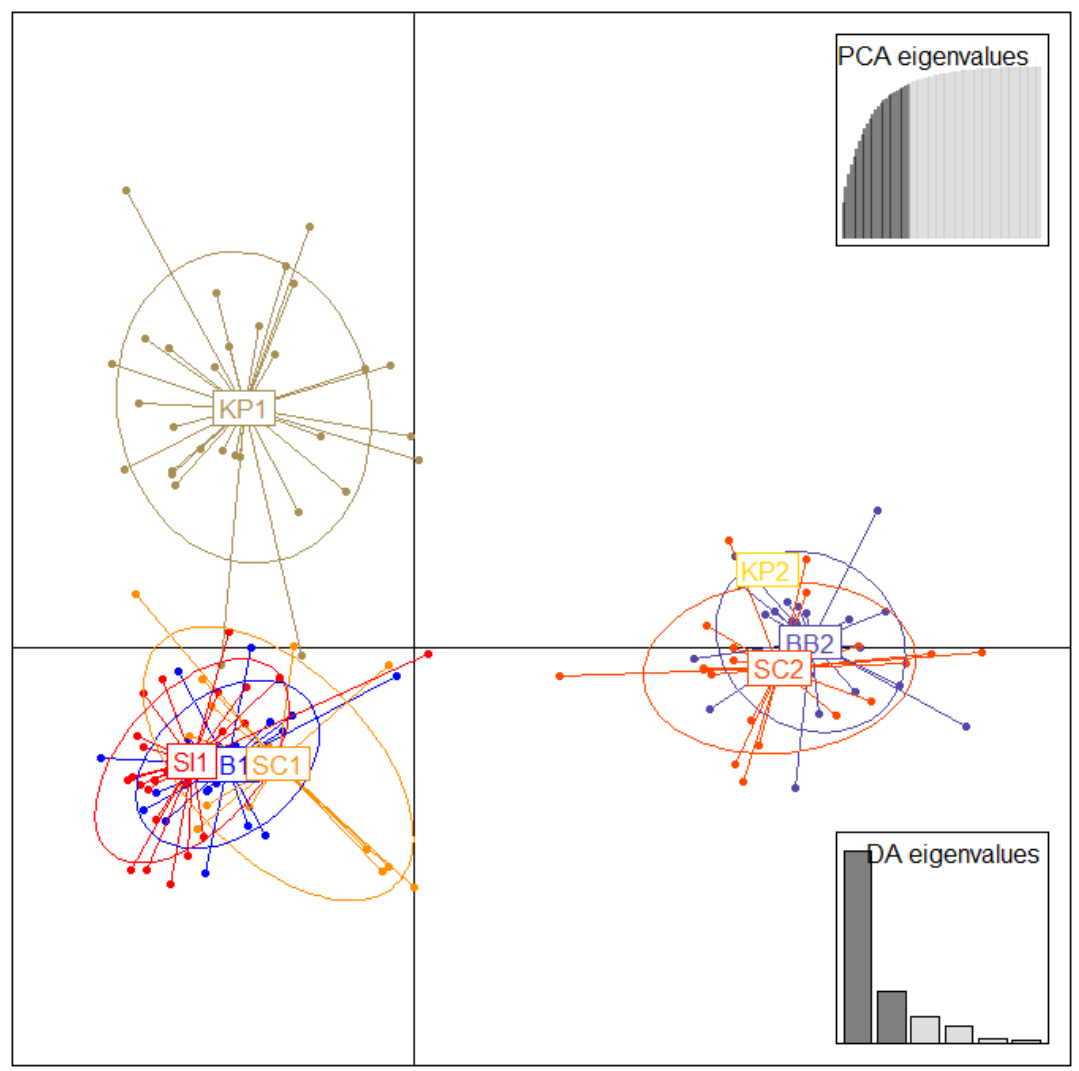

Figure 6.3. Genetic division between the Tethya burtoni complex, where population codes are: $\mathrm{BB}=$ Breaker Bay, $\mathrm{SC}=$ South Coast, $\mathrm{SI}=$ Matiu-Somes Island, KP $=$ Kapiti Island. Those populations with a ' 1 ' belonged to Clade 2 produced by $\mathrm{rnl} / 18 \mathrm{~S}$ and those with a ' 2 ' belonged to Clade 3 produced by rnl/18S (see Chapter 2, Figures $2.2 \& 2.3$ ). Each point in the discriminant principal coordinate analysis represents a sponge genotype which are encompassed within $67 \%$ of their population inertia ellipse. 
In addition to collecting sponges from central New Zealand for this thesis, I also collected samples from both northern New Zealand (Poor Knights, collected under permit number 52367-MAR, issued by the Department of Conservation) and southern New Zealand (Fiordland). The microsatellite loci failed to consistently amplify in these sponges (i.e., only one or two loci amplified for some individuals). Failed amplifications can result from mutations in the primer binding site or divergence between two species. Many descriptions of Tethya in New Zealand are based from observations in northern New Zealand. For instance, the holotype for T. burtoni was collected from Tauranga, northern New Zealand, and descriptions were made in conjunction with approximately 10 other specimens viewed from the same area (Sarà and Sarà, 2004). It is important to note, however, that species described in northern New Zealand may not occupy the whole of New Zealand and instead, have genetically diverged from their so called conspecifics occupying other regions, as evidenced through both the observed failed amplification of microsatellites and the structured populations across small distances observed in Chapter 5. Indeed, more genetic analyses would shed more light onto the geographic barriers and divergence of Tethya across New Zealand. It is highly probable, however, there is greater diversity within this genus than what is currently recorded in the literature.

\section{Evolutionary forces shaping Tethya populations: connectivity, cryptic speciation and introgression}

Failing to detect cryptic species can have serious consequences for the interpretation of connectivity data, as exemplified by this thesis. For example, if the population structure analyses were run for $T$. burtoni without consideration for potential cryptic speciation within the samples (i.e., including all samples that were a priori thought to be $T$. burtoni), then the connectivity patterns would appear different to that of just one species within the T. burtoni complex (Figure 6.4A versus Figure 6.4B). In the case without recognizing cryptic speciation, the number of recognized populations (genetic clusters, K) from STRUCTURE decreased from three to two compared to considering just one species, where each of the clusters refers to one of the species. When $\mathrm{K}=2$, Matiu-Somes Island appears more genetically similar to Kapiti (Figure 6.4B). If the number of genetic clusters is increased to 6 for this analysis (to account for population locations, Figure $6.4 \mathrm{C}$ ), more structure is revealed attributable to population structure within each species, but the resulting plot is more confusing to interpret. In this case, 

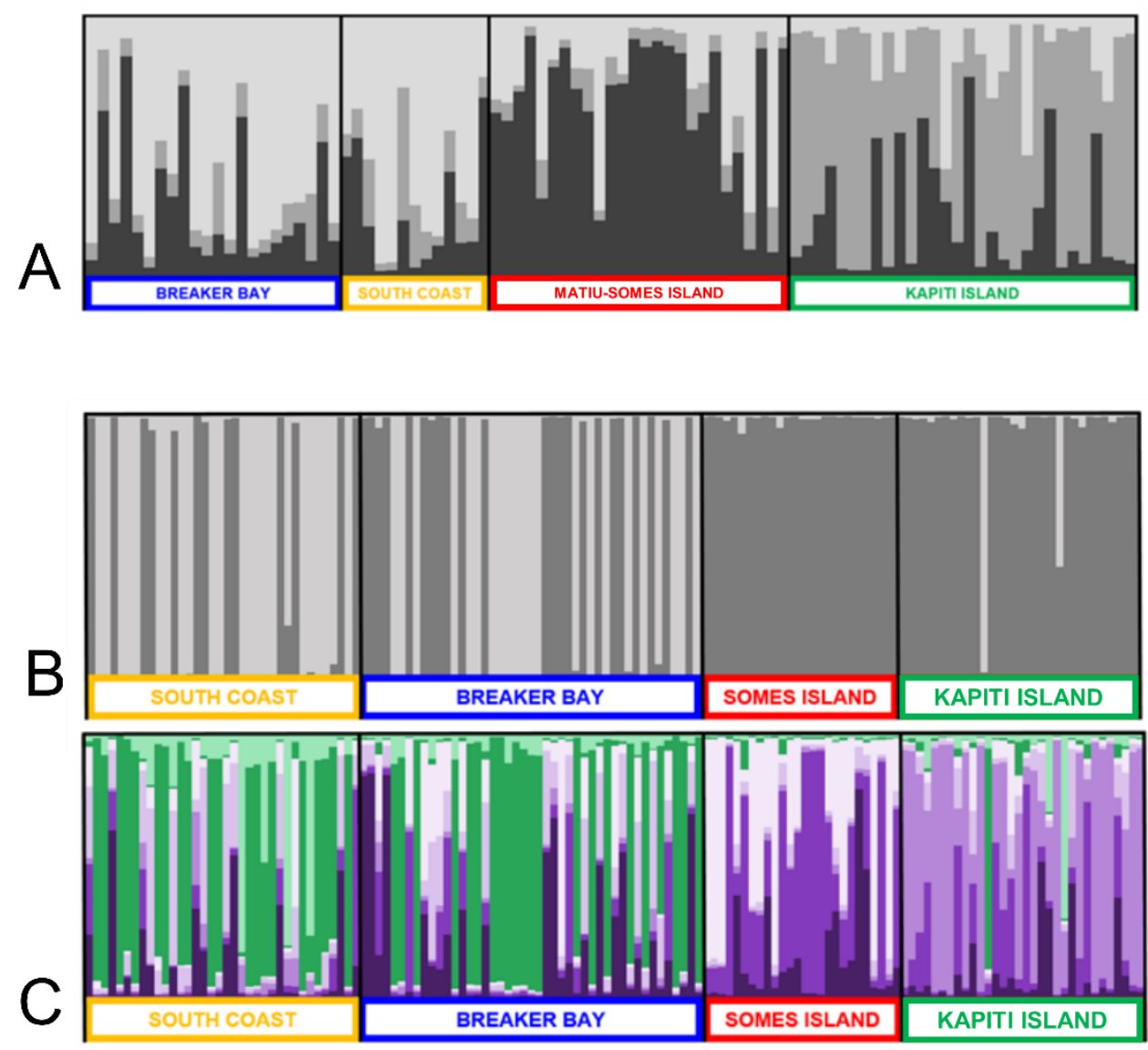

Figure 6.4. STRUCTURE plots comparing population structure of only one species of $T$. burtoni (A, plot from Chapter 5, Figure 5.4) to the T. burtoni complex (B \& C, new plots). Each vertical line corresponds to a sponge, and the proportion of colour is the probability of assignment into that colour's genetic cluster $(K)$. For plot $A, K=3$. For plot $B, K=2$ and for plot $C, K=6$. For plots $B+C$, the plots contain the same individuals in the same order, and plots are aligned so that the same individual can be read from B directly down to C.

Matiu-Somes Island and Kapiti Island appear to be the most genetically similar, despite the fact that they are both isolated populations that are the furthest away from each other. For this study, the effects of cryptic speciation on predicting connectivity patterns could eventually be disentangled, but for larger studies with greater sample sizes and more locations, failure to correctly identifying species may not be as obvious and could lead to serious erroneous interpretation of connectivity patterns. The design of marine reserves is often based on demographic and genetic connectivity data (Roberts, 1997; Palumbi, 2003), and therefore failure to correctly identify such patterns may be particularly detrimental, both economically and ecologically. For example, species complexes have already been identified for commercially important species including squid (Triantafillos and Adams, 2005), rockfish (Creamer, 2015), croaker (Cooke et al., 2011), and lobster (Tourinho et al., 2012), as well as for pharmaceutically important sponges (Wörheide et al., 2008; Anjum et al., 2016). Failure to 
identify cryptic species may lead to the creation of incorrect catch quotas for certain species and may result in overfishing, exploitation, reduction of diversity and lead to overall population collapse.

Cryptic speciation often occurs when species have limited connectivity between populations across large geographical distances, thus leading to allopatric genetic divergence and speciation (Knowlton, 1993; Bickford et al., 2007). Genetic evidence has revealed that many organisms that are considered cosmopolitan (i.e., morphologically similar species living worldwide) are actually comprised of multiple cryptic species. For instance, Barroso et al. (2010) found that the so-called cosmopolitan polychaete Eurythoe complanata actually consists of three genetically different species that belong to different regions (Pacific, Caribbean Sea and South Atlantic). Similarly, high degrees of genetic divergence between populations of the calcareous sponge Leucetta chagosensis from various Indo-Pacific populations reveal likely cryptic speciation within the group, where morphological differences between such genetically different populations were not obvious (Wörheide et al., 2008). The opposite, however, was observed in this study for sponges belonging to what is currently called T. burtoni, where speciation occurs for species living in the same location. The STRUCTURE plot containing both species reveals that the cryptic species mainly occur at Breaker Bay and the South Coast populations, which were well connected populations. No cryptic species were recorded at Matiu-Somes Island, and further, only one of the other species was found at Kapiti, suggesting that some barriers to gene flow exist that are preventing these species expanding their range or successfully settling in these locations.

High gene flow between Breaker Bay and the South Coast coupled with high genetic diversity/differentiation seems contradictory, but could occur under different scenarios. For instance, allopatric speciation may occur to form cryptic species in the first instance (Lande, 1980), and then barriers to gene flow that allowed divergence of the populations may change (e.g., topographic changes after seismic activity, or change in currents). This change would result in gene flow to be re-established between diverged populations, where two species remain reproductively isolated from one another (Rüber et al., 2001; Barluenga and Meyer, 2004). Another scenario that would support high gene flow with cryptic speciation is speciation occurring while species are coexisting in the same habitat (termed sympatric speciation). In 
such a circumstance, each species may diverge if they find a slightly different niche and have an 'adaptive-shift', which could occur in the presence of high levels of gene flow (Faucci et al., 2007; Niemiller et al., 2008). It is unclear as to the process by which the T. burtoni complex has come to live in sympatry with gene flow still occurring between some populations. The high frequency of earthquakes and the changing coastal seabed in New Zealand (Berryman, 1993; Hayward et al., 2006; Carter, 2008) could be a possible route by which gene flow is reestablished for populations that have diverged allopatrically. New Zealand exhibits a strong north-south differentiation in genetic structure for many coastal organisms (Ross et al., 2009; Gardner et al., 2010). In the Cook Strait region of central New Zealand, haplotypes belonging to both North and South Island populations have been recorded for chitons (Veale and Lavery, 2011). Both cryptic species of T. burtoni observed in this present study may be a mixing of north and south populations. A more extensive study surveying north and south populations would be needed to better support this hypothesis. Nonetheless, by determining both the timing of speciation versus the timing of gene flow, as well as possible restrictions to gene flow that may foster divergence (Nosil 2008), it may become clear if the evolutionary history follows the first or second scenario for the T. burtoni complex.

When examining the STRUCTURE plot for both species, there is one individual in the Kapiti population whose assignment is $50 \%$ into one cluster (species) and $50 \%$ into the other. This provides evidence of a hybrid individual, which would allow the potential for introgressive hybridization to occur between species. Further, the discordance of the phylogenetic trees generated from different markers when delineating species (Chapter 2) could be suggestive that some sponges may have undergone introgression (though diploid markers would be needed to confirm this notion). Cross fertilization between congeneric gametes as well as fusion between genetically different larvae have been recorded for sponges and coral that exhibit synchronous spawning (i.e., Maldonado, 1998; Hatta et al., 1999). Introgression and hybridization have been recorded for other many sessile marine taxa, including sponges (Riesgo et al., 2016), corals (Frade et al., 2010; Hellberg et al., 2016), seaweeds (Coyer et al., 2007), ascidians (Roux et al., 2013), bivalves (Bierne et al., 2003), and also for motile organisms like fish (Verspoor and Hammart, 1991; Harrison et al., 2017) and crustaceans (Darling, 2011). The overlap in timing of sexual reproduction between $T$. bergquistae and $T$. burtoni observed in this study (see Chapter 3) may provide a mechanism by which introgression can occur, as gametes from different species occur in the water column simultaneously, which may promote cross- 
fertilization. Interestingly, those T. burtoni that showed evidence of possible instances of introgressive hybridization were generally from Kapiti and Matiu-Somes Island. Both of these populations experienced more restricted gene flow from the panmictic Breaker Bay and South Coast populations, and further, did not show high instances of cryptic speciation. The frequency of introgression may be positively associated with mate availability. For instance, because both cryptic species occur at Breaker Bay and the South Coast populations, gametes would have a higher chance of finding conspecific relatives. At both Kapiti and Matiu-Somes Islands, however, the lower presence of one of the cryptic species may result in higher instances of introgression due to the fact that gametes cannot find conspecific gametes for fertilization. Low levels of gene flow coupled with local retention of genotypes in these populations may only result in a small number of cryptic species entering the gene pool of these populations. However, without other available mates, such species would likely be either outcompeted or undergo introgression with heterospecific gametes. Willis et al. (2011) have recorded that a decrease in the rate of mate encounters increased the chance of hybridization for fish. Further, outcrossing in flowers decreased when more flowers were open on the same stem compared to surrounding flowers (Cruzan et al., 1994). Introgression in relation to mate availability in sponges has not been previously recorded, and while the restricted gene flow observed here may limit available mates and lead to instances of introgression, this hypothesis warrants more investigation.

\section{Species-specific responses}

Comparison of sexual reproduction in Tethya versus other sponges from the temperate Australasian region

Comparing reproduction between species living in similar environments may shed light on how organisms have differentially adapted reproductive strategies. This is especially true for organisms living in sympatry, where different strategies may permit an understanding of how such species coexist in an environment where they occupy the same niche. Table 6.1 shows information from reproductive studies conducted on sponges in temperate Australasia, which are characterized by rocky reefs, kelp forests and cyclic temperature patterns. This table allows comparison of reproductive timing, gamete production and development, gamete density, and gamete reproductive output index (ROI) for sponges existing in this temperate region. When examining sponges from the Australasian temperate region (Table 6.1), no trends aside from 
Table 6.1. Information on reproduction for sponge species from temperate Australasia. Time refers to reproductive timing, which is either discrete/seasonal (S) or continuous (C). Gamete production (prod) refers to either gonochorism $(\mathrm{G})$ or hermaphroditism $(\mathrm{H})$, and gamete development (dev) refers to either ovipary $(\mathrm{O})$ or vivipary $(\mathrm{V})$. Reproductive output index (ROI) refers to the proportion of sponge occupied by gametes (given as \%). Densities given in counts (oocytes, embryos, larvae or spermatic cysts) per $\mathrm{mm}^{2}$, and all data were adjusted to be presented in $\mathrm{mm}^{2}$. Ranges given for each numerical column, except when only one number presented, which is the maximum value observed for that sponge. $\mathrm{NF}$ refers to gametes not being found, and - refers to information not available. For location, NZ = New Zealand and W-AU = Western Australia.

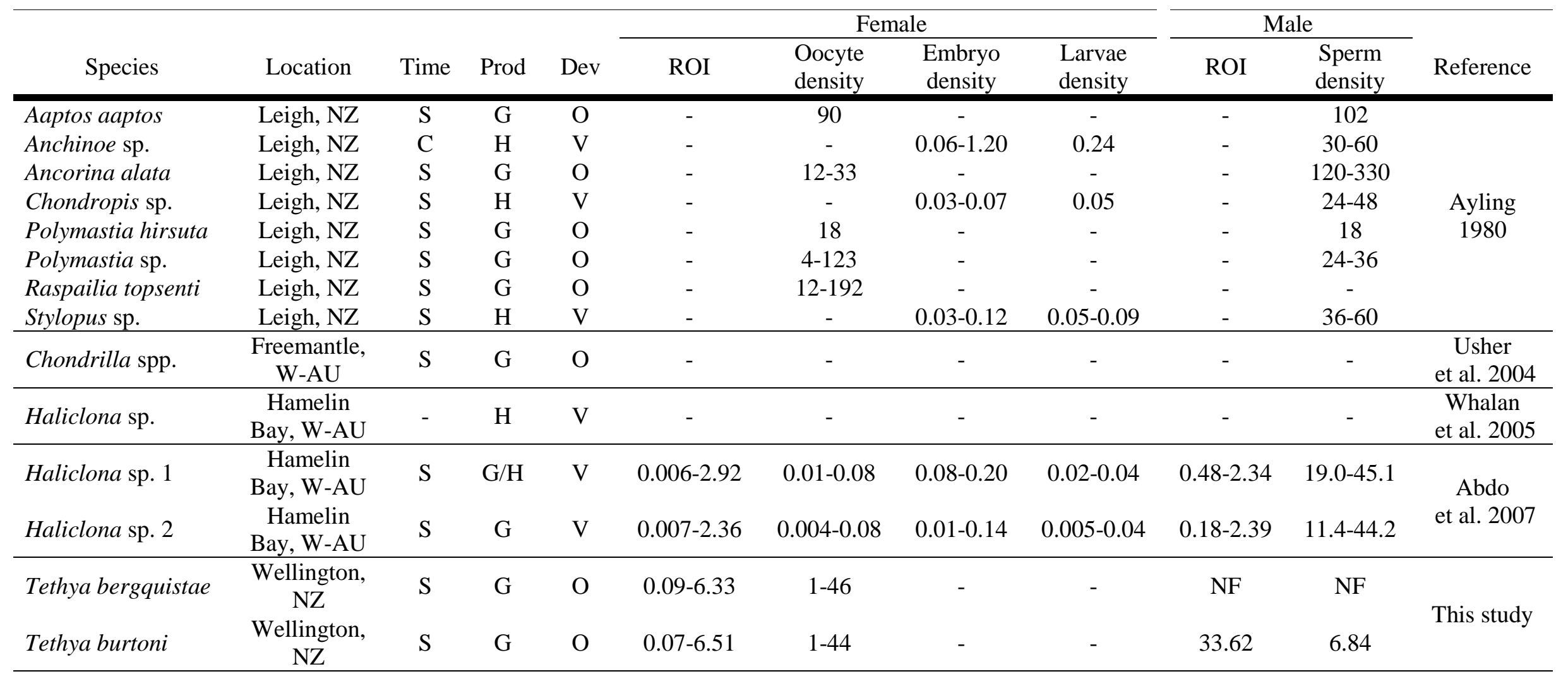


seasonality of reproductive timing are apparent. About half of the sponges are hermaphroditic and viviparous, while the other half are gonochoristic and oviparous, suggesting selection for a certain reproductive strategy in this environment may be more heavily reliant on intrinsic species-specific adaptations. Tethya spp. were gonochoristic and oviparous, and the maximum oocyte density we observed for Tethya sp. $\left(\sim 45\right.$ oocytes $\left./ \mathrm{mm}^{2}\right)$ was lower than maximum oocyte densities for other sponges. For instance, Raspailia topsenti had much higher oocyte density $\left(\sim 192\right.$ oocytes $\left./ \mathrm{mm}^{2}\right)$. It is important to note that the observed densities may be a product of sampling points. In this study, it is likely that the period of maximum oocyte density, which would likely occur between February and March before gamete release, was missed during my sampling regime. This is apparent when examining densities of oocytes in Tethya bergquistae during the 2016 sexual reproductive period. Oocyte density increased from January to February, but then decreased from February to March, indicating that the release of gametes had begun between the sampling of February and March. More frequent sampling would better resolve maximum oocyte densities capable in Tethya spp. and may give a better indication of the overall fecundity of Tethya, which may allow a better comparison to other sponges inhabiting similar habitats.

Another explanation for reduced oocyte density observed in Tethya compared to other sponges may be due to simultaneous sexual and asexual reproduction. Interestingly, both asexual and sexual reproduction occurred during the austral summer, and some individuals that were found to contain oocytes also contained asexual buds. Energetic costs of simultaneously producing buds may decrease the available energy for sexual reproduction, resulting in a lower observed fecundity. For most organisms, asexual reproduction occurs during periods when sexual reproduction has ceased, resulting in the alternate employment of each mode (Haven, 1971; Corriero et al., 1996; Conand et al., 2002, Simon et al., 2002). Here, however, budding occurred continuously throughout the year and with the highest proportion of budding sponges and the highest bud densities in spring and further coinciding with the time of sexual reproduction. The simultaneous production of both sexual larvae and asexual propagules in nature is rare, as both modes generally have different cues. Conbosch and Vollmer (2013) found that the coral Pocillopora damicornis participates in mixed reproduction, where they produced both sexual and asexual larvae at the same time. The authors suggest such a mixed reproductive strategy is evolutionary advantageous in that if offers a 'best of both worlds scenario,' where successful genotypes engaged in sexual reproduction are amplified through 
asexual reproduction at the same time that such alleles are passed to future generations through sexual reproduction. This may also be true for Tethya spp. The reduced sexual reproductive ability observed in clonal Tethya may be a way by which a high number of clonal organisms can exist without negative effects of inbreeding. In this way, advantageous genotypes could continually become amplified by asexual reproduction, but the sexual reproductive ability of the parent is not compromised by sexually reproducing with itself. This may prove advantageous in varying environments, where sexual reproduction would allow new alleles to adapt to changes and asexual reproduction would allow successful genotypes to persist.

\section{Tethya bergquistae as a more thermally tolerant sponge than T. burtoni}

This $\mathrm{PhD}$ thesis highlighted species-specific responses in reproduction for two species of Tethya that occupy the same environment and are seemingly subject to the same environmental forces. Interestingly, in the experiment investigating the effects of temperature on asexual reproduction, survivability between both species markedly varied (Figure 6.5 A,B). For $T$. bergquistae, both the control and $19^{\circ} \mathrm{C}$ treatment resulted in greater than $90 \%$ survivability, and the $21^{\circ} \mathrm{C}$ treatment resulted in $70 \%$ survivability. For T. burtoni, by contrast, the control had two deaths occurring near the end of the experiment ( $80 \%$ survivability), but the warmer treatments both saw almost complete mortality by the end of the experiment. Standard Kaplan Meir survival curves were constructed for each species in each treatment (Figure 6.5 C,D), which are nonparametric analyses that estimate the survival function from incomplete data (Kaplan and Meier, 1958; Efron, 1987). Kaplan Meir curves allow estimation of survival times and generally the median (i.e., the time at which the survival probability is less than $50 \%$ ) is used to compare different curves. In this case, T. bergquistae did not reach a median survival time nor did the T. burtoni control, so I used the first quartile (Q1) to compare species, which is defined as the time when the survival probability reaches $75 \%$ and less (Table 6.2). The control for both groups had the same Q1 survival time (15 days), but T. burtoni had a reduced survival time compared to T. bergquistae for the higher temperature treatments. As such, it appears T. bergquistae has a higher thermal tolerance than T. burtoni.

To put the observed experimental mortality rates into context, data from the field collected from monitoring data revealed that both species in situ have a similar mortality rate. For instance, over the two years monitoring, $18 \%$ of tagged T. bergquistae died and $17 \%$ of $T$. 

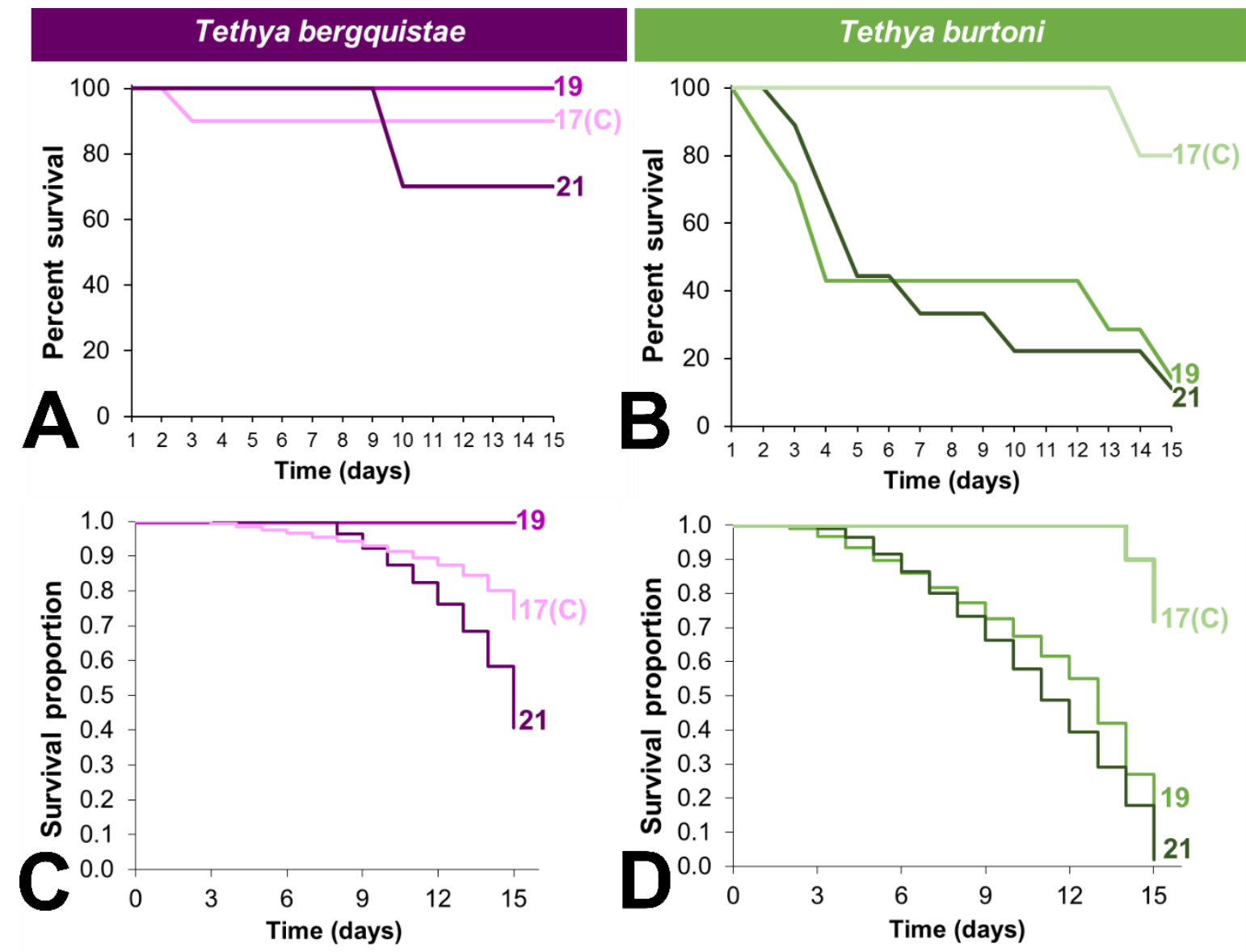

Figure 6.5. Percent survival for Tethya bergquistae and T. burtoni at different temperature treatments (A-B) and associated Kaplan Meier survival curves (C-D). The control temperature was the $17^{\circ} \mathrm{C}$ treatment, indicated by the symbol '(C)'.

burtoni died. The controls exhibited similar mortality rates. The differences in observed mortality between thermal treatments, however, are indicative that species may not respond in the same way to thermal stress. It appears that $T$. burtoni may have more difficulty adapting to rapid changes in temperature higher than those experienced in nature, as most mortality occurred within the first 5 days. Other differences in species habitat may have amplified thermal stress and caused a faster mortality rate for T. burtoni. For instance, T. burtoni generally lives in more sedimented areas and for this experiment, there were low volumes of sediment. Therefore, experimental conditions could also have accounted for differences in mortality. Nonetheless, there appears to be species-specific responses to thermal stress for Tethya, where intrinsic characteristics exist that allow each species to adapt differentially. Differences in physiological responses to various stressors have also been observed for a number of other sponges (Bennett et al., 2016; Bates, 2017), as well as other marine 
invertebrates (e.g., Heyward and Negri, 2010). Understanding how different species will respond differently to changing environments is important in predicting changes in ecosystems dynamics, as climate change will likely lead to shifts in the community/ecosystem composition based on the adaptability of particular species.

Table 6.2. Number of days calculated from Kaplan Meier survival curves predicted for $25 \%$ of the population to die, for comparisons between different temperature treatments and Tethya spp.

\begin{tabular}{ccc}
\hline & T. bergquistae & T. burtoni \\
\hline Control & 15 days & 15 days \\
19 & $>15$ days & 9 days \\
21 & 13 days & 8 days \\
\hline
\end{tabular}

\section{Changes in population dynamics as a result of climate change}

From this $\mathrm{PhD}$ thesis, a holistic view has emerged of evolutionary forces shaping population dynamics, particularly with regard to species boundaries and connectivity, and environmental drivers and roles of asexual and sexual reproduction for Tethya spp. With such information, it is possible to make predictions on changes in population dynamics for this sponge under different future climate scenarios. Future environmental changes projected by the IPCC are expected to cause range shifts and the advancement of spring events, and many marine and terrestrial species have already been reported as being affected by climate change (IPCC, 2014; Parmesan and Yohe, 2003; Edwards and Richardson, 2004; Visser and Both, 2005; Sherry et al., 2007; Inouye, 2008). For instance, Parmesan and Yohe (2003) conducted a meta-analysis on over 650 different marine and terrestrial species, and found that nearly half of them already showed some evolutionary responses to anthropogenic climate change, including shifts in phenology, species range and abundance. Temperate systems rely on seasonal changes, which drive the cyclic patterns of productivity that foster growth at higher trophic levels, and for this reason, are said to be most vulnerable to changes in the environment (Edwards and Richardson, 2004; Vergés et al., 2014). A mismatch has already been recorded between primary productivity/nutrient availability and phenology of pelagic organisms at higher trophic levels (Edwards and Richardson, 2004). Organisms physiologically respond to thermal stress; and 
further, temperature and primary production in temperate systems are important drivers for cyclic phenology. Therefore, changes in both of these environmental conditions are likely to alter population dynamics for many temperate organisms, particularly if they cannot adapt quickly enough.

For New Zealand, warming trends have been inconsistent throughout the country for the past century. Due to changes in the circulation of the South Pacific subtropical gyre, southern New Zealand has seen the greatest warming over the past 50-100 years (Shears and Bowen, 2017). Northern and central New Zealand have experienced less warming. The findings of Shears and Bowen (2017) suggest that if warming continues in the same manner for the next century, there will be a homogenization of the marine environment. Currently, New Zealand ranges from subtropical to subpolar, but with comparatively greater warming in the south versus other regions, all of New Zealand is likely to lose diversity and instead become a similar marine environment throughout. Predictions have been made for New Zealand in the next century using simulations with different IPCC scenarios, and show that sea surface temperatures are projected to increase over $2^{\circ} \mathrm{C}$ by 2100 , while levels of both primary production and macronutrients are predicted to decrease by $\sim 5-20 \%$ (Law et al., 2017). Central regions are expected to be less affected by climate change than other regions (i.e., the Chatham Rise, subpolar areas south of $50^{\circ} \mathrm{C}$, subtropical waters of Northern New Zealand; Law et al., 2017). Therefore, while these sponges may be affected by climate change, they may be better placed compared to other sponges occupying these more at-risk regions of New Zealand.

Temperature was found to be correlated with both seasonal sexual and asexual reproduction in Tethya bergquistae and T. burtoni, and as such, shifts in phenology for both of these modes of reproductive are probable with changes in season. Shifts in seasons, where the onset of cooling (winter) occurs later than typical, has been recorded as already occurring in New Zealand (Shears and Bowen, 2017). Seasonal changes may result in extended, shortened or shifted periods of reproduction; and/or increased or reduced fecundity. Furthermore, though not directly correlated to chl- $a$ concentration, reproduction occurred after periods of high productivity. Changes to both timing and levels of primary productivity and macronutrients are likely to have detrimental effects on reproduction in these sponges, which seemingly rely on seasonal fluxes of food. If shifts in the reproductive phenology of Tethya do not match changes 
in the environment caused by climate change, then a mismatch is likely to not only have negative consequences for Tethya, but also have cascading effects throughout the system. Sponges play many key functional roles on reefs, especially in habitat creation, water filtration, and trophic interactions (Bell, 2008). In this study, sea stars and nudibranchs were observed feeding on Tethya (M. Shaffer, personal observation, Figure 6.6), indicating changes in abundance of Tethya could have trophic consequences. Both modes of reproduction were shown to play different roles in the population dynamics for Tethya, where sexual reproduction was important for connectivity and asexual reproduction for population maintenance. Both modes of reproduction occurred simultaneously, giving an indication that the success of one
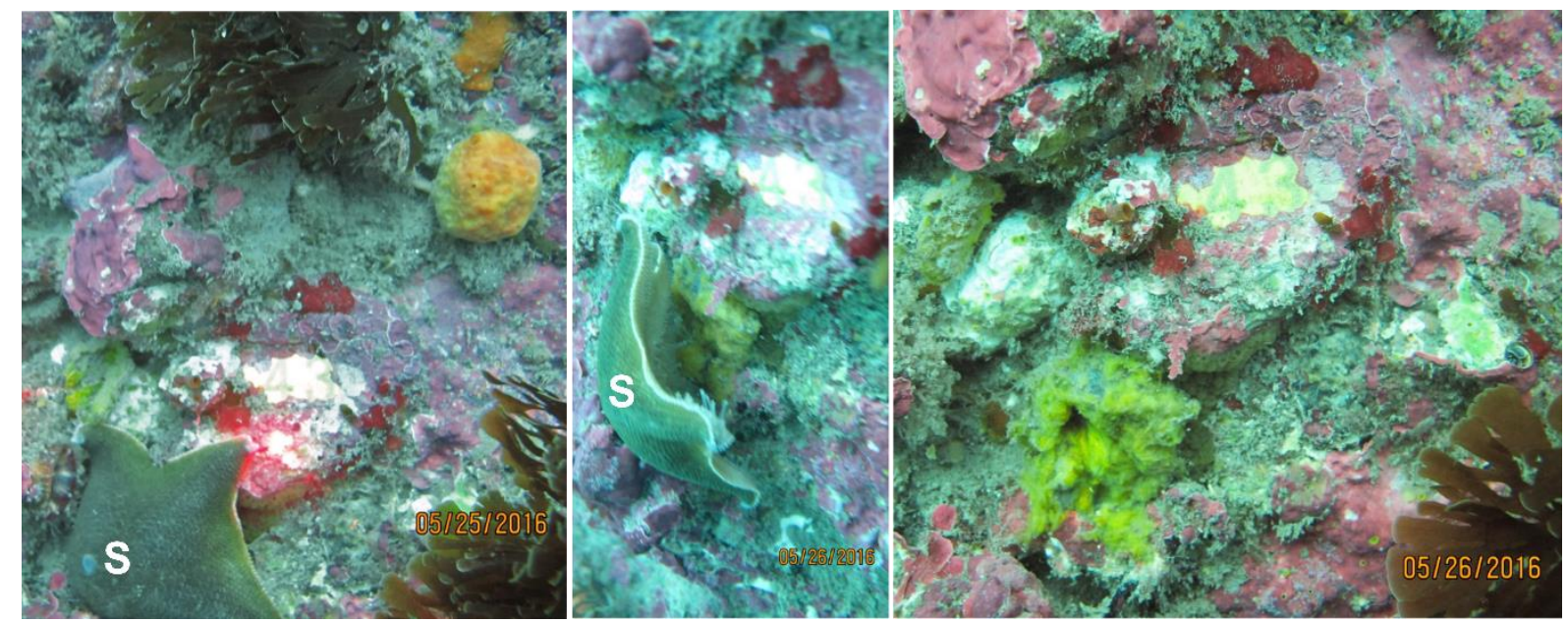

Figure 6.6. Observations over two days of a sea star (S) preying on Tethya burtoni, where the picture on the right shows remains of digested sponge tissue.

mode may rely or be dependent on the other mode. Mismatch not only between reproduction and the environment, but also between sexual and asexual reproduction, may also have genetic consequences. For instance, if buds play a key role in parental survivorship, but instances of asexual reproduction decrease or are timed differently, the capacity of parental sponges to sexually reproduce may be greatly reduced. Such reduction in genetic diversity may lead to less adaptability potential for the population. In addition, if water movement drives asexual reproduction and storms/swell increase, there may be a higher instance of buds that may outcompete larvae. If fitness differences exist between both sexually reproduced and clonal offspring (especially under different stressors), population abundance, distribution and connectivity may be altered. Experimental studies revealed that increased temperature may 
particularly be more detrimental for T. burtoni, and thus under warming oceans, $T$. bergquistae may be able to better cope with thermal stress and outcompete T. burtoni. These results are important for predicting future population viability, especially as such changes are likely to have cascading effects to other levels of the ecosystem.

\section{Future directions}

This research is relevant to many other marine species that share similar life history characteristics with Tethya, namely sympatry, sexual and asexual reproduction, cryptic speciation and introgression. As such, these results allow a greater understanding of complex evolutionary processes that shape marine populations, which are often difficult to study and understand in the marine environment. Tethya spp. have proven to be excellent models to examine evolutionary questions and could be utilized further to address more complex questions. This work, therefore, has future directions in the following areas: clarifying the taxonomic literature for Tethya; assessing the response of larvae versus buds to environmental stressors; and the use of microsatellites for parentage analyses and to investigate instances of hybridization, introgression and chimerism.

\section{Taxonomic clarifications}

New Zealand is known for hosting a highly diverse sponge assemblage (Bergquist and Warne, 1968), and for Tethya in particular, the number of species in New Zealand and Australia is higher than anywhere else in the world (Bergquist and Kelly-Borges, 1991; Sarà and Sarà, 2004). Tethya is a ubiquitous genus found in tropical, temperate and polar waters (Carter, 1872; Corriero et al., 1989; Sarà and Bavestrello, 1994; Heim et al., 2007), though compared to other areas of the world, little genetic work has been performed looking at phylogenetic relationships for Tethya in New Zealand. Understanding species boundaries in this particular hotspot may allow a better understanding for the evolution of other species worldwide. Furthermore, finding certain factors that may support diversity in these waters requires a proper cataloguing of species in order to capture true species delineations and therefore true estimates of diversity. The genus Tethya in particular has been shown to be of pharmaceutical value (Kurelec and Pivcevic, 1992; Wiese et al., 2011; Gentile et al., 2016), and so understanding the ecology, species boundaries and true diversity of these species may be beneficial from both an ecological 
and economic perspective. The current taxonomic classifications of Tethya in New Zealand would benefit from phylogenetic reconstructions. Specifically, extracting DNA from holotypes, coupled with specimen collected throughout New Zealand, may better address the extent of gene flow for these species across New Zealand and reveal the true diversity for Tethya. Furthermore, analyses of a morphological character set in conjunction with genetic analyses may be able to characterize the extent of cryptic speciation within the group in New Zealand and worldwide, and provide a set of diagnostic traits.

\section{Fitness of larvae versus buds}

Studies have shown that thermal stress may increase larval mortality, reduce fertilization rates and increase larval abnormalities for corals (Edmunds et al., 2001; Negri et al., 2007). For sponges, increased temperature has also been shown to reduce the duration of the larval planktonic stage, resulting in the potential for more subdivided, genetically distinct populations (Whalan et al., 2008). Sponge larvae, however, have also been recorded to exhibit higher levels of tolerance to increased temperature than other marine taxa (Webster et al., 2011). Compared to sexually derived larvae, there is currently little research on the response of asexually reproduced individuals to environmental stressors. There is evidence that sexual lineages and asexual lineages may have different tolerances and adaptations (Tooley et al., 1986; Browne et al., 1988; Amat et al., 2006), and therefore offspring of both modes are likely to be differentially affected by stressors. While this thesis suggested under what conditions both sexual and asexual reproduction may be favourable, a complete picture of how populations dynamics may change under changing environments is lacking. Specifically, there remain gaps in our knowledge as to whether differential responses of offspring of both sexual and asexual reproduction exist. By examining fitness levels of sexually reproduced offspring versus asexually reproduced offspring in response to various environmental stressors, predictions about the genetic structure and overall population growth under different climate scenarios may be able to be better made. As an example, if thermal stress is more detrimental to asexually reproduced offspring compared to sexually reproduced offspring, then the number of clones produced may decrease. If clones play an important role in genotype survivorship and population persistence, as suggested in this thesis (Chapter 5), then sexually reproduced individuals may also suffer, leading to an overall population decline. There is already evidence that the fitness of clones of T. burtoni is reduced compared to non-clones, evidenced by their 
reduced sexual reproductive ability (Chapter 5) and increased mortality rate (40\% versus 20\%). As such, it is highly probable that other differences in fitness also exist. Tethya spp. have offered an excellent model organism for this thesis to explore hypotheses relating to reproduction, and they would further prove beneficial in exploring these questions relating to fitness differences in sexually reproduced offspring/larvae and asexually reproduced offspring/buds. Microsatellites developed in this thesis would allow for sexually reproduced offspring to be distinguished from asexually reproduced offspring. Further, data provided in Chapters 3 and 4 about reproductive timing may allow both larvae and buds to be reared in the lab and experiments conducted. As differential responses between sexual and asexual lineages have the potential to result in changes to genetic diversity and connectivity patterns, experiments could further be carried out with clonal populations versus diverse populations to determine how genetic diversity may affect population growth and individual fitness. As genetic diversity has previously been associated with increased population viability (Williams, 2001), such results have important implications when considering the implementation of management plans to conserve biodiversity.

\section{Parentage analyses, hybridization, introgression, chimerism: microsatellites as a tool}

Parentage analyses allow many evolutionary questions to be answered, especially relating to patterns of dispersal, sexual selection, settlement success rate and recruitment processes (Jones et al., 2010). The microsatellite markers developed in this thesis could be further utilized for a parentage analyses, and would allow genetic structure and recruitment of a population to be explored at a finer scale. Tethya in particular serve as useful models to investigate questions surrounding parentage assignment because they are easy to monitor and new recruits settle and are visible within the span of a year (M. Shaffer, personal observation).

There is currently evidence of hybridization, introgression and chimerism in the sponge phylum (Maldonado, 1998; Riesgo et al., 2016). Hybridization, or when two different species mate and produce an individual that contains the genetic makeup from both species, is more common than previously thought in marine environments (Gardner, 1997; Riesberg, 1998). The use of the microsatellite markers developed in this thesis can further investigate the extent of hybridization between Tethya species, as the results of this thesis suggest it occurs in these sponges. Further, the microsatellites developed for this thesis, in combination with diploid 
nuclear markers, can be used to investigate introgression (which occurs when hybrids cross back with one of the species, incorporating part of the other species genome into the other) and determine when evolutionarily such introgression events occurred (Heiser, 1949; Heiser, 1973). For instance, hybrids indicated from microsatellite data may be suggestive of a more recent or contemporary hybridization event (due to the fast evolving nature of microsatellites), whereas hybrids indicated from slower evolving nuclear or mitochondrial DNA may suggest a past introgressive event. Lastly, chimerism, or when two individuals that contain different genotypes fuse together to result in one individual with discrete patches of each genotype (Sommerfeldt et al., 2003; Puill-Stephan et al., 2009), may be a likely phenomenon that occurs in these Tethya species. Fusion events, which could be a mechanism for chimerism, were commonly observed for Tethya throughout the study period for this thesis. The microsatellite markers could be employed after field- and lab-based experiments of fusion events to determine the extent of chimerism and address long standing evolutionary questions as to the benefits of chimerism (Maldonado, 1998; Ben-Shlomo, 2017).

\section{Conclusion}

This $\mathrm{PhD}$ thesis provides baseline data for population dynamics that occur for Tethya spp. and uses genetic tools to elucidate how sympatric species of sessile marine organisms, like sponges, evolve. In particular, it investigated species boundaries and connectivity patterns for the temperate sponges Tethya bergquistae and T. burtoni on New Zealand reefs; and further, characterized the phenology of both sexual and asexual reproduction, while determining potential environmental drivers of both modes of reproduction. Climate change is threatening marine ecosystems worldwide, and understanding basic organismal processes like reproduction is imperative for predicting how marine populations may cope with changing environments. Furthermore, many marine invertebrates share life-history traits with sponges and may evolve in a similar way, and these results allow the elucidation of complex evolutionary processes such as cryptic speciation and introgression. Together, this thesis allows a thorough understanding of population dynamics for Tethya and may aid in creating conservation and management plans that must take these processes into consideration. 


\section{REFERENCES}

Abdo DA, Fromont J, McDonald JI (2008) Strategies, patterns and environmental cues for reproduction in two temperate haliclonid sponges. Aquatic Biology, 1(3), 291-302.

Abdul Wahab MA, de Nys R, Holzman R, Schneider CL, Whalan S (2017) Patterns of reproduction in two co-occurring Great Barrier Reef sponges. Marine and Freshwater Research, 68, 1233-1244.

Abdul Wahab MA, De Nys R, Webster N, Whalan S (2014) Phenology of sexual reproduction in the common coral reef sponge, Carteriospongia foliascens. Coral Reefs, 33, 381-394.

Adlard RD, Lester JG (1995) Development of a diagnostic test for Mikrocytos roughleyi, the aetiological agent of Australian winter mortality of the commercial rock oyster, Saccostrea commercialis (Iredale \& Roughley). Journal of Fish Disease, 18, 609-614.

Alberto F, Gouveia L, Arnaud-Haond S, Perez-Llorens JL, Duarte CM, Serrao EA (2005) Within-population spatial genetic structure, neighbourhood size and clonal subrange in the seagrass Cymodocea nodosa. Molecular Ecology, 14(9), 2669-2681.

Alvarez B, Crisp MD, Driver F, Hooper JNA, Van Soest RWM (2000) Phylogenetic relationships of the family Axinellidae (Porifera: Demospongiae) using morphological and molecular data. Zoologica Scripta, 29, 169-198.

Amano S (1986) Larval release in response to a light signal by the intertidal sponge Halichondria panicea. The Biological Bulletin, 171, 371-378.

Amano S (1988) Morning release of larvae controlled by the light in an intertidal sponge, Callyspongia ramosa. Biological Bulletin, 175, 181-184.

Amat I, Castelo M, Desouhant E, Bernstein C (2006) The influence of temperature and host availability on the host exploitation strategies of sexual and asexual parasitic wasps of the same species. Oecologia, 148(1), 153-161.

Amato A, Kooistra WHCF, Ghiron JHL, Mann DG, Proschold T, Montresor M (2007) Reproduction isolation among sympatric cryptic species in marine diatoms. Protist, 158(2), 193-207.

Andersson, MB (1994) Sexual Selection. Princeton University Press.

Andreakis N, Luter HM, Webster NS (2012) Cryptic speciation and phylogeographic relationships in the elephant ear sponge Ianthella basta (Porifera, Ianthellidae) from northern Australia. Zoological Journal of the Linnean Society, 166, 225-235.

Anjum K, Abbas SQ, Shah SAA, Akhter N, Batool S, Hassan SSU (2016) Marine sponges as a drug treasure. Biomolecules and Therapeutics, 24(4), 347-362.

Anne C (2006) Choosing the right molecular genetic markers for studying biodiversity: from molecular evolution to practical aspects. Genetica, 127, 101-120. 
Antao T, Lopes A, Lopes RJ, Beja-Pereira A, Luikart G (2008) LOSITAN: a workbench to detect molecular adaptation based on a $F_{\mathrm{ST}}$-outlier method. BMC Bioinformatics, 9, 323.

Apte S, Gardner JPA (2002) Population genetic subdivision in the New Zealand greenshell mussel (Perna canaliculus) inferred from single-strand conformation polymorphism analysis of mitochondrial DNA. Molecular Ecology, 11, 1617-1628.

Arnaud-Haond S, Duarte CM, Alberto F, Serrao EA (2007) Standardizing methods to adddress clonality in population studies. Molecular Ecology, 16(24), 5115-5139.

Arnaud-Haond S, Migliaccio M, Diaz-Almela E, Teixeira S, Van De Vliet MS, Alberto F, Procaccini G, Duarte CM, Serrao EA (2007) Vicariance patterns in the Mediterranean Sea: east-west cleavage and low dispersal in the endemic seagrass Posidonia oceania. Journal of Biogeography, 34(6), 963-976.

Arnaud-Haond S, Teixeira S, Massa SI, Billot C, Saenger P, Coupland G, Durante CM, Serrao EA (2006) Genetic structure at range edge: low diversity and high inbreeding in Southeast Asian mangrove (Avicennia marina) populations. Molecular Ecology, 15(12), 3515-3525.

Avise JC (2012) Molecular Markers, Natural History and Evolution. Springer Science \& Business Media: New York.

Ayling AL (1980) Patterns of sexuality, asexual reproduction and recruitment in some subtidal marine demospongiae. The Biological Bulletin, 158(3), 271-282.

Ayre DJ (1984) The effects of sexual and asexual reproduction on geographic variation in the sea anemone Actinia tenebrosa. Oecologia, 62(2), 222-229.

Ayre DJ, Hughes TP (2000) Genotypic diversity and gene flow in brooding and spawning corals along the Great Barrier Reef, Australia. Evolution, 54(5), 1590-1605.

Ayre DJ, Resing JM (1985) Sexual and asexual production of planulae in reef corals. Marine Biology, 90(2), 187-190.

Bailey KM, Picquelle SJ (2002) Larval distribution of offshore spawning flatfish in the Gulf of Alaska: potential pathways and enhanced onshore transport during ENSO events. Marine Ecology Progress Series, 236, 205-217.

Baldacconi R, Nonnis-Marzano C, Gaino E, Corriero G (2007) Sexual reproduction, larval development and release in Spongia officinalis L. (Porifera, Demospongiae) from the Apulian coast. Marine Biology, 152, 969-979.

Ballinger GA (2004) Using generalized estimating equations from longitudinal data analysis. Organizational Research Methods, 7(2), 127-150.

Balloux F, Lugon-Moulin N (2002) The estimation of population differentiation with microsatellite markers. Molecular Ecology, 11, 155-165. 
Barbuti R, Mautner S, Carnevale G, Milazzo P, Rama R, Sturmbauer C (2012) Population dynamics with a mixed type of sexual and asexual reproduction in a fluctuating environment. BMC Evolutionary Biology, 12(2), 49.

Barluenga M, Meyer A (2004) The Midas cichlid species complex: incipient sympatric speciation in Nicaraguan cichlid fishes? Molecular Ecology, 13, 2061-2076.

Barroso R, Klautau M, Solé-Cava A, Paiva PC (2010) Eurythoe complanata (Polychaeta: Amphinomidae), the 'cosmopolitan' fireworm, consists of at least three cryptic species. Marine Biology, 157(1), 69-80.

Barton NH, Charlesworth B (1998) Why sex and recombination? Science, 281(5385), 19861990.

Bates T (2015) Sponge physiology and function in a changing ocean: responses to ocean acidification and increased sea surface temperature. MSc Thesis, Victoria University of Wellington, Wellington, New URI: http://restrictedarchive.vuw.ac.nz/handle/123456789/9051.

Bates TEM, Bell JJ (2017) Responses of two temperate sponge species to ocean acidification. New Zealand Journal of Marine and Freshwater Research. 52(2), 247-263.

Battershill CN, Bergquist PR (1990) The influence of storms on asexual reproduction, recruitment and survivorship of sponges. In: Ruetzler K (Ed.) New Perspectives in Sponge Biology. Smithsonian Institution Press: Washington, D.C., USA; London, England, UK, pp. 397-403.

Battershill CN, Bergquist PR, Cook SDC (2010) Phylum Porifera. In: Cook SDC (Ed.) New Zealand Coastal Marine Invertebrates, Canterbury University Press: Christchurch, New Zealand. Vol 1, pp 122-124.

Baum DA, Shaw KL (1995) Genealogical perspectives on the species problem. In: Hoch PC, Stevenson AG (Eds.) Experimental and Molecular Approaches to Plant Biosystematics, Missouri Botanical Garden: St. Louis, Vol 53, pp 289-303.

Baums IB, Miller MW, Hellberg ME (2006) Geographic variation in clonal structure in a reefbuilding Caribbean coral, Acropora palmata. Ecological Monographs, 76(4), 503-519.

Bavestrello G, Bonito M, Sará M (1993) Influence on depth on the size of sponge spicules. Scientia Marina, 57, 415-420.

Beauchamp KA (1992) Gametogenesis, brooding and planulation in laboratory populations of a temperate scleractinian coral Balanophyllia elegans maintained under contrasting photoperiod regimens. Invertebrate Reproduction and Development, 23(2-3), 171-182.

Becks L, Agrawal AF (2010) Higher rates of sex evolves in spatially heterogeneous environments. Nature, 468, 89-92.

Becks L, Agrawal AF (2012) The evolution of sex is favoured during adaptation to new environments. PLoS Biology, 10(5), e1001317. 
Belinky F, Szitenberg A, Goldfarb I, Feldstein T, Wörheide G, Ilan M, Huchon D (2012) ALG11 - A new variable DNA marker for sponge phylogeny: Comparison of phylogenetic performances with 18S rDNA and the COI gene. Molecular Phylogenetics and Evoltuion, 63, $702-713$.

Bell JJ (2008) The functional roles of marine sponges. Estuarine, Coastal and Shelf Science, 79(3), 341-353.

Bell J, Barnes D, Turner J (2002) The importance of micro and macro morphological variation in the adaptation of a sublittoral demosponge to current extremes. Marine Biology, 140, 75-81.

Bell JJ, McGrath E, Biggerstaff A, Bates T, Bennett H, Marlow J, Shaffer M (2015) Sediment impacts on marine sponges. Marine Pollution Bulletin, 94, 5-13.

Bengtsson BO (2003) Genetic variation in organisms with sexual and asexual reproduction. Journal of Evolutionary Biology, 16(2), 189-199.

Bennett HM (2017) Climate change and tropical sponges: the effect of elevated $\mathrm{pCO}_{2}$ and temperature on the sponge holobiont. PhD Thesis, Victoria University of Wellington, Wellington, New Zealand. URI: http://hdl.handle.net/10063/6240.

Bennett HM, Altenrath C, Woods L, Davy SK, Webster NS, Bell JJ (2016) Interactive effects of temperature and $\mathrm{pCO}_{2}$ on sponges: from the cradle to the grave. Global Change Biology, 23(5), 2031-2046.

Ben-Shlomo R (2017) Invasiveness, chimerism and genetic diversity. Molecular Ecology, 26(3), 6502-6509.

Bentley MG, Olive PJW, Last K (2001) Sexual satellites, moonlight and the nuptial dances of worms: the influence of the moon on the reproduction of marine animals. Earth, Moon and Planets, 85-86, 67-84.

Bergquist PR (1961) Demospongiae (Porifera) of the Chatham Islands and Chatham Rise, collected by the Chatham Islands 1954 Expedition. New Zealand Oceanographic Institute Memoir, 13, 169-206.

Bergquist PR (1978) Sponges. University of California Press, USA.

Bergquist PR, Kelly-Borges M (1991) An evaluation of the genus Tethya (Porifera: Demospongiae: Hadromerida) with descriptions of new species from the southwest Pacific. The Beagle, Records of the Northern Territory Museum of Arts and Sciences, 8, 37-72.

Bergquist PR, Warne KP (1968) The marine fauna of New Zealand: Porifera, Demospongiae. Department of Scientific and Industrial Research, Vol 1.

Bergquist PR, Sinclair ME (1968) The morphology and behaviour of larvae of some intertidal sponges. New Zealand Journal of Marine and Freshwater Research, 2, 426-437. 
Berman J (2012) Patterns of temporal and spatial variability of sponge assemblages. PhD Thesis, Victoria University of Wellington, Wellington, New Zealand. URI: http://hdl.handle.net/10063/2061.

Berntson EA, Bayer FM, McArthur AG, France SC (2001) Phylogenetic relationships within the Octocorallia (Cnidaria: Anthozoa) based on nuclear 18S rRNA sequences. Marine Biology, $138,235-246$.

Berryman K (1993) Age, height and deformation of Holocene marine terraces at Mahia Peninsula, Hikurangi Subduction Margin, New Zealand. Tectonics, 12(6), 1347-1364.

Bickford D, Lohman DJ, Sodhi NS, Ng PKL, Meier R, Winker K, Ingram KK, Das I (2007) Cryptic species as a window on diversity and conservation. Trends in Ecology and Evolution, 22(3), 148-155.

Bierne N, Borsa P, Daguin C, Jollivet D, Viard F, Bonhomme F, David P (2003) Introgression patterns in the mosaic hybrid zone between Mytilus edulis and M. galloprovincialis. Molecular Ecology, 12(2), 447-461.

Black R, Johnson MS (1979) Asexual viviparity and population genetics of Actinia tenebrosa. Marine Biology, 53(1), 27-31.

Blanquer A, Uriz MJ (2007) Cryptic speciation in marine sponges evidenced by mitochondrial and nuclear genes: A phylogenetic approach. Molecular Phylogenetics and Evolution, 45, 392397.

Blanquer A, Uriz MJ (2010) Population genetics at three spatial scales of a rare sponge living in fragmented habitats. BMC Evolutionary Biology, 10, 13.

Blanquer A, Uriz MJ, Caujape-Castells J (2009) Small-scale spatial genetic structure in Scopalina lophyropoda, an encrusting sponge with philopatric larval dispersal and frequent fission and fusion events. Marine Ecology Progress Series, 380, 95-102.

Bond C (1992) Continuous cell movements rearrange anatomical structures in intact sponges. Journal of Experimental Zoology Part A: Ecological and Integrative Physiology, 263, 284-302.

Boore JL (1999) Animal mitochondrial genomes. Nucleic Acids Research, 27, 1767-1780.

Borchiellini C, Chombard C, Lafay B, Boury-Esnault N (2000) Molecular systematics of sponges (Porifera). Hydrobiologia, 420(1), 15-27.

Bowman MJ, Foster BA, Lapennas PP (1983) Ocean water properties (Cook Strait). In: Maui Development Environmental Study Report on Phase 2 1977-1981. Report prepared by the University of Auckland for Shell BP and Todd Oil Services Ltd.

Box GEP, Tidwell PW (2012) Transformation of the independent variables. Technometrics, 4(4), 531-550. 
Bradford JM, Roberts PE (1978) Distribution of reactive phosphorus and plankton in relation to upwelling and surface circulation around New Zealand. New Zealand Journal of Marine and Freshwater Research, 12(1), 1-15.

Bronstein O, Loya Y (2015) Photoperiod, temperature, and food availability as drivers of the annual reproductive cycle of the sea urchin Echinometra sp. from the Gulf of Aqaba (Red Sea). Coral Reefs, 34(1), 275-289.

Browne RA, Davis LE, Sallee SE (1988) Effects of temperature and relative fitness of sexual and asexual brine shrimp Artemia. Journal of Experimental Marine Biology and Ecology, 124(1), 1-20.

Bucklin A, Steinke D, Blanco-Bercial L (2011) DNA barcoding of marine metazoan. Annual Review of Marine Science, 3, 471-508.

Burdon JJ, Roefls AP (1985) The effect of sexual and asexual reproduction on the isozyme structure of populations of Puccinia graminis. Phytopathology, 75, 1068-1073.

Bürger R (1999) Evolution of genetic variability and the advantage of sex and recombination in changing environments. Genetics, 153(2), 1055-1069.

Byrne M (2011) Impact of ocean warming and ocean acidification on marine invertebrate life history stages: vulnerability and potential for persistence in a changing ocean. Oceanography and Marine Biology: An Annual Review, 49, 1-42.

Byrne M, Ho M, Selvakumaraswamy P, Nguyen HD, Dworjanyn SA, Davis AR (2009) Temperature, but not $\mathrm{pH}$, compromises sea urchin fertilization and early development under near-future climate change scenarios. Proceedings of the Royal Society B: Biological Sciences, 276(1663), 1883-1888.

Capa M, Pons J, Hutchings P (2013) Cryptic diversity, intraspecific phonetic plasticity and recent geographical translocations in Branchiomma (Sabellidae, Annelida). Zoological Scripta, 42, 637-655.

Capel KCC, Toonen RJ, Rachid CTCC, Creed JC, Kitahara MV, Forsman Z, Zilberberg C (2017) Clone wars: asexual reproduction dominates in the invasive range of Tubastraea spp. (Anthozoa: Scleractinia) in the South-Atlantic Ocean. PeerJ, 5, e3873.

Cardone F, Gaino E, Corriero G (2010) The budding process in Tethya citrina Sarà \& Melone (Porifera, Demospongiae) and the incidence of post-buds in sponge population maintenance. Journal of Experimental Marine Biology and Ecology, 389(1-2), 93-100.

Cardone F, Nonnis Marzano C, Spedicato MT, Lembo G, Gaino E, Corriero G (2008) Budding induction in a marine sponge: a cue for cultivation purposes. Proceedings of IFOAM Conference on Organic Aquaculture, Cattolica, 81 - 85 .

Carlos L, Taboada S, Combosch D, Giribet G, Riesgo A (2017) Population connectivity of Dendrilla antarctica Topsent, 1905 (Porifera, Demospongiae) from west Antarctica shallow bottoms using Genome-wide SNPs obtained from RADseq techniques. XIIth SCAR Biology Symoposium, 59. 
Carter L (2008) Below low tide - a seabed in motion. In: Gardner J, Bell JJ (2008) The Taputeranga Marine Reserve. First Edition Ltd: Wellington, NZ, pp. 130-140.

Carter HJ (1872) On two new sponges from the Antarctic Sea, and on a new species of Tethya from Shetland; together with observations on the reproduction of sponges commencing from zygosis of the sponge animal. The Annals and Magazine of Natural History, 9, 409-435.

Charlesworth B (1980) The cost of sex in relation to mating system. Journal of Theoretical Biology, 84(4), 655-671.

Charlesworth D, Wright SI (2001) Breeding systems and genome evolution. Current Opinion in Genetics \& Development, 11(6), 685-690.

Chaves-Fonnegra A, Feldheim KA, Secord J, Lopez JV (2015) Population structure and dispersal of the coral-excavating sponge Cliona delitrix. Molecular Ecology, 24(7), 1447-1466.

Clarke KR (1993) Non-parametric multivariate analyses of changes in community structure. Australian Journal of Ecology, 18, 117-143.

Coffroth MA, Lasker HR (1998) Population structure of a clonal gorgonian coral: the interplay between clonal reproduction and disturbance. Evolution, 52(2), 379-393.

Combosch DJ, Vollmer SV (2013). Mixed and asexual reproduction in the Indo-Pacific reef coral Pocillopora damicornis. Ecology and Evolution, 3(10), 3379-3387.

Conand C, Uthicke S, Hoareau T (2002) Sexual and asexual reproduction of the holothurian Stichopus chlorontus (Echinodermata): a comparison between La Reunion (Indian Ocean) and east Australia (Pacific Ocean). Invertebrate Reproduction and Development, 41(1-3), 235-242.

Cooke GM, Chao NL, Beheregaray LB (2011) Marine incursions, cryptic species and ecological diversification in Amazonia: the biogeographic history of the croaker genus Plagioscion (Sciaenidae). Journal of Biogeography, 39(4), 724-738.

Corriero G, Balduzz A, Sarà M (1989) Ecological differences in the distribution of two Tethya (Porifera, Demospongiae) species coexisting in a Mediterranean coastal lagoon. Marine Ecology, 10(4), 303-315.

Corriero G, Gadaleta F, Bavestrello G (2015) A new Mediterranean species of Tethya (Porifera: Tethyida: Demospongiae). Italalian Journal of Zoology, 82, 535-543.

Corriero G, Sara M, Vaccaro P (1996) Sexual and asexual reproduction in two species of Tethya (Porifera: Demospongiae) from a Mediterranean coastal lagoon. Marine Biology, 126(2), 175-181.

Corriero G, Scalera Liaci L, Nonnis-Marzano C, Gaino E (1998) Reproductive strategies of Mycale contarenii (Porifera: Demospongiae). Marine Biology, 131, 319-327.

Cowen RK, Gawarkiewicz G, Pineda J, Thorrold SR, Werner FE (2007) Population connectivity in marine systems: an overview. Oceanography, 20(3), 14-21. 
Cowen RK, Lwiza KMM, Sponaugle S, Paris CB, Olson DB (2000) Connectivity of marine populations: open or closed? Science, 287(5454), 857-859.

Cowen RK, Sponaugle S (2009) Larval dispersal and marine population connectivity. Annual Review of Marine Science, 1, 443-466.

Coyer JA, Hoarau G, Stam WT, Olsen JL (2007) Hybridization and introgression in a mixed population of the intertidal seaweeds Fucus evanescens and F. serratus. Journal of Evolutionary Biology, 20(6), 2322-2333.

Creamer JM (2016) Using genetic species identification and environmental data to distinguish historical catches of cryptic Blackspotted Rockfish (Sebastes melanostictus) and Rougheye Rockfish (Sebastes aleutianus) in British Colombia. A thesis submitted to the University of Georgia. URL: http://summit.sfu.ca/item/16709.

Crespo A, Pérez-Ortega S (2009) Cryptic species and species pairs in lichens: a discussion on the relationship between molecular phylogenies and morphological characters. Anales del Jardin Botanico de Madrid, 66(S1), 71-81.

Crow JF (1992) An advantage of sexual reproduction in a rapidly changing environment. Journal of Heredity, 83(3), 169-173.

Cruzan MB, Hamrick JL, Arnold ML, Bennett BD (1994) Mating system variation in hybridizing irises: effects of phenology and floral densities on family outcrossing rates. Heredity, 72, 95-105.

Cruz-Barraza JA, Carballo JL, Rocha-Olivares A, Ehrlich H, Hog M (2012) Integrative Taxonomy and Molecular Phylogeny of Genus Aplysina (Demospongiae: Verongida) from Mexican Pacific. PLoS One, 7, e42049.

Czarna A, Gawrońska B, Nowińska R, Morozowska M, Kosiński P (2016) Morphological and molecular variation in selected species of Erysimum (Brassicaceae) from Central Europe and their taxonomic significance. Flora, 222, 68-85.

Dailianis T, Tsigenopoulos CS, Dounas C, Voultsiadou E (2011) Genetic diversity of the imperilled bath sponge Spongia officinalis Linnaeus, 1759 across the Mediteranean Sea: patterns of population differentiation and implications for taxonomy and conservation. Molecular Ecology, 20(18), 3757-3772.

Daly M (1978) The cost of mating. The American Naturalist, 112(968), 771-774.

Darling JA (2011) Interspecific hybridization and mitochondrial introgression in invasive Carcinus shore crabs. PLoS One, 6(3), e17828.

De Almeida LD, Netto EBF, Coutinho R (2017) Inter-annual cascade effect on marine food web: A benthic pathway lagging nutrient supply to pelagic fish stock. PLoS One, 12(9), $\mathrm{e} 0184512$.

De Meeûs T, Balloux F (2004) Clonal reproduction and linkage disequilibrium in diploids: a simulation study. Infection, Genetics and Evolution, 4(4), 345-351. 
De Paula TS, Zilberberg C, Haidu E, Lobo-Haidu G (2012) Morphology and molecules on opposite sides of the diversity gradient: four cryptic species of the Cliona celata (Porifera, Demospongiae) complex in South America revealed by mitochondrial and nuclear markers. Molecular Phylogenetics and Evolution, 62, 529-541.

De Queiroz K (2007) Species concepts and species delimitation. Systematic Biology, 56, 879886.

DeBiasse MB, Hellberg ME (2015) Discordance between morphological and molecular species boundaries among Caribbean species of the reef sponge Callyspongia. Ecology and Evolution, 5, 663-675.

DeBiasse MB, Richards VP, Shivji MS (2010) Genetic assessment of connectivity in the common reef sponge, Callyspongia vaginalis (Demospongiae: Haploscerida) reveals high population structure along the Florida reef tract. Coral Reefs, 29(1), 47-55.

Delmotte F, Leterme N, Gauthier JP, Ripse C, Simon JC (2002) Genetic architecture of sexual and asexual populations of the aphid Rhopalosiphum padi based on allozyme and microsatellite markers. Molecular Ecology, 11(4), 711-723.

Derycke S, Vanaverbeke J, Rigaux A, Backeljau T, Moens T (2010) Exploring the use of cytochrome oxidase c subnit 1 (COI) for DNA barcoding of free-living marine nematodes. PLoS One, 5, e13716.

DeSalle R, Egan MG, Siddall M (2005) The unholy trinity: taxonomy, species delimitation and DNA barcoding. Philosophical Transactions of the Royal Society B, 360, 1905-1916.

Di Camillo CG, Coppari M, Bartolucci I, Bo M, Betti F, Bertolino M, Calcinai B, Cerrano C, De Grandis G, Bavestrello G (2012) Temporal variations in growth and reproduction of Tedania anhelans and Chondrosia reniformis in the North Adriatic Sea. Hydrobiologia, 687, 299-313.

Doncaster CP, Pound GE, Cox SJ (2000) The ecological cost of sex. Nature, 404, 281-285.

Duckworth AR (2003) Effect of wound size on the growth and regeneration of two temperate subtidal sponges. Journal of Experimental Biology and Ecology, 287(2), 139-153.

Duckworth AR, Battershill CN (2001) Population dynamics and chemical ecology of New Zealand Demospongiae Latruncuila sp. nov. and Polymastia croceus (Poecilosclerida: Latrunculiidae: Polymastiidae). New Zealand Journal of Marine and Freshwater Research, $35: 5,935-949$.

Duckworth AR, Battershill CN, Schiel DR (2004) Effects of depth and water flow on growth, survival and bioactivity of two temperate sponges cultured in different seasons. Aquaculture, $242,237-250$.

Duran S, Pascual M, Estoup A, Turon X (2004b) Strong population structure in the marine sponge Crambe (Poecilosclerida) as revealed by microsatellite markers. Molecule Ecology, 13(3), 511-522. 
Duran S, Pascual M, Turon X (2004a) Low levels of genetic variation in mtDNA sequences over the western Mediterranean and Atlantic range of the sponge Crambe (Poecilosclerida). Marine Biology, 144(1), 31-35.

Durant JM, Hjermann DO, Ottersen G, Stenseth NC (2007) Climate and the match or mismatch between predator requirements and resource availability. Climate Research, 33(3), 271-283.

Earl DA, VonHoldt BM (2012) STRUCTURE HARVESTER: a website and program for visualizing STRUCTURE output and implementing the Evanno method. Conservation Genetics Resources, 4(2), 359-361.

Edmunds R, Gates R, Gleason D (2001) The biology of larvae from the reef coral Porites astreoides, and their response to temperature disturbances. Marine Biology, 139(5), 981-989.

Edwards M, Richardson AJ (2004) Impact of climate change on marine pelagic phenology and trophic mismatch. Nature, 430, 881-883.

Efron B (1987) Logistic regression, survival analysis, and the Kaplan-Meier curve. Journal of the American Statistical Association, 83, 402.

Ellstrand NC, Roose ML (1987) Patterns of genotypic diversity in clonal plant species. American Journal of Botany, 74(1), 123-131.

Elvin DW (1976) Seasonal growth and reproduction of an intertidal sponge, Haliclona permollis (Bowerbank). The Biological Bulletin, 151, 108-125.

Engelstädter J (2008) Constraints on the evolution of asexual reproduction. BioEssays, 30, 1138-1150.

Epherra L, Damián DG, Rubilar T, Perez-Gallo S, Reartes MB, Tolosano JA (2015) Temporal and spatial differences in the reproductive biology of the sea urchin Arbacia dufresnii. Marine and Freshwater Research, 66, 329-342.

Ereskovsky AV (2000) Reproduction cycles and strategies of the cold-water sponges Halisarca dujardini (Demospongiae, Halisarcida), Myxilla incrustans and Iophon piceus (Demospongiae, Poecilosclerida) from the White Sea. Biological Bulletin, 198, 77-87.

Ereskovsky AV, Dubois M, Ivanišević J, Gazave E, Lapebie P, Tokina D, Pérez T (2013) Pluriannual study of the reproduction of two Mediterranean Oscarella species (Porifera, Homoscleromorpha): cycle, sex-ratio, reproductive effort and phenology. Marine Biology, 160(2), 423-438.

Erpenbeck D, Breeuwer JAJ, Parra-Velandia FJ, Van Soest RWM (2006b) Speculation with spiculation? Three independent gene fragments and biochemical characters versus morphology in demosponge higher classification. Molecular Phylogenetics and Evolution, 38, 293-205.

Erpenbeck D, Duran S, Rutzler K, Paul V, Hooper JNA, Wörheide G (2007) Towards a DNA taxonomy of Caribbean demosponges: a gene tree reconstructed from partial mitochondrial $\mathrm{CO} 1$ gene sequences supports previous rDNA phylogenies and provides a new perspective on 
the systematics of Demospongiae. Journal of the Marine Biological Association of the United Kingdom, 87, 1563-1570.

Erpenbeck D, Hooper JNA, Wörheide G (2006a) CO1 phylogenies in diploblasts and the 'Barcoding of Life' - are we sequencing a suboptimal partition? Molecular Ecology Notes, 6, $550-553$.

Erwin PM, Thacker RW (2007) Phylogenetic analyses of marine sponges within the order Verongida: a comparison of morphological and molecular data. Invertebrate Biology, 126, 220234.

Ettinger-Epstein P, Whalan SW, Battershill CN, de Nys R (2007) Temperature cues gametogenesis and larval release in a tropical sponge. Marine Biology, 153, 171-178.

Evanno G, Regnaut S, Goudet J (2005) Detecting the number of clusters of individuals using a software STRUCTURE: a simulation study. Molecular Ecology, 14(8), 2611-2620.

Excoffier L, Laval G, Schneider S (2005) Arlequin ver. 3.0: An integrated software package for population genetics data analysis. Evolutionary Bioinformatics Online, 1, 47-50.

Faucci A, Toonen RJ, Hadfield MG (2007) Host shift and speciation in a coral-feeding nudibranch. Proceedings of the Royal Society B: Biological Sciences, 274, 111-119.

Fell PE (1974) Diapause in the gemmules of the marine sponge, Haliclona loosanoffi, with a note on the gemmules of Haliclona oculata. The Biological Bulletin, 147(2), 333-351.

Fell PE (1976) The reproduction of Haliclona loosanoffi and its apparent relationship to water temperature. The Biological Bulletin, 150(2), 200-210.

Folmer O, Black M, Hoeh W, Lutz R, Vrijenhoek R (1994) DNA primers for amplification of mitochondrial cytochrome $\mathrm{C}$ oxidase subunit I from diverse metazoan invertebrates. Molecular Marine Biology and Biotechnology, 3, 294-299.

Frade PR, Reyes-Nivia MC, Faria J, Kaandorp JA, Luttikhuizen PC, Bark RP (2010) Semipermeable species boundaries in the coral genus Madracis: introgression in a brooding coral system. Molecular Phylogenetics and Evolution, 57(3), 1072-1090.

Frankham R, Ballou JD, Dudash MR, Eldridge MDB, Fenster CB, Lacy RC, Mendelson JR III, Porton IJ, Ralls K, Ryder OA (2012) Implications of different species concepts for conserving biodiversity. Biological Conservation, 153, 25-31.

Fromont J, Bergquist PR (1994) Reproductive biology of three sponge species of the genus Xestospongia (Porifera: Demospongiae: Petrosida) from the Great Barrier Reef. Coral Reefs, 13(2), 119-126.

Frost TM (1976) Sponge feeding: a review with a discussion of some continuing research. Aspects of Sponge Biology, 283-298.

Gabriel W, Lynch M, Bürger R (1993) Muller's ratchet and mutational meltdowns. Evolution, 47, 6, 1744-1757. 
Gaggiotti OE, Lange O, Rassman K, Gliddon C (1999) A comparison of two indirect methods of estimating average levels of gene flow using microsatellite data. Molecular Ecology, 8(9), 1513-1520.

Gaino E, Burlando B, Buffa P, Sarà M (1987) Ultrastructural study of the mature egg of Tethya citrina Sarà and Melone (Porifera, Demospongiae). Gamete Research, 16, 259-265.

Gaino E, Cardone F, Corriero G (2010) Reproduction of the intertidal sponge Hymeniacidon perlevis (Montagu) along a bathymetric gradient. The Open Marine Biology Journal, 4, 47-56.

Gaino E, Manconi R, Pronzato R (1995) Organizational plasticity as a successful conservative tactics in sponges. Animal Biology 4, 31-43.

Gaino E, Sarà M (1994) An ultrastructural comparative study of the eggs of two species of Tethya (Porifera, Demospongiae). Invertebrate Reproduction and Development, 26(2), 99-106.

Gaino E, Scalera Liaci L, Sciscioli M, Corriero G (2006) Investigation of the budding process in Tethya citrina and Tethya aurantinium (Porifera, Demosgponiae). Zoomorphology, 125, 8797.

Galarza JA, Carreras-Carbonell J, Macpherson E, Pascual M, Roques S, Turner GF, Rico C (2009) The influence of oceanographic fronts and early-life-history traits on connectivity among littoral fish species. Proceedings of the National Academy of Sciences of the United States of America, 106(5), 1473-1478.

Gardner JPA (1997) Hybridization in the sea. Advances in Marine Biology, 31, 1-78.

Gardner JPA, Bell JJ, Constable HB, Hannan D, Ritchie PA, Zuccarello GC (2010) Multispecies coastal marine connectivity: a literature review with recommendations for further research. New Zealand Acquatic Environment and Biodiversity Report No. 58.

Gardner JPA, Pande A, Eyles RF, Wear WG (1996) Biochemical genetic variation among populations of the greenshell mussel, Perna canaliculus, from New Zealand: preliminary findings. Biochemical Systematics and Ecology, 24, 763-774.

Gasparini F, Manni L, Cima F, Zaniolo G, Burighel P, Caicci F, Franchi N, Schiavon F, Rigon $\mathrm{R}$, Campagna D, Ballarin L (2015) Sexual and asexual reproduction in the colonial ascidian Botryllus schlosseri. Genesis, 53(1), 105-120.

Gentile E, Marzano CN, Latronico T, Fasano A, Rossano R, Corriero G, Luizzi GM (2016) Bioassay-guided identification of bioactive protein compounds from the Mediterranean demosponge Tethya meloni. Planta Medica, 82(S01), S1-S381.

Gerrish PJ, Lenski RE (1998) The fate of competing beneficial mutations in an asexual population. Genetica, 102, 127.

Ghisletta P, Spini D (2004) An introduction to generalized estimating equations and an application to assess selectivity effects in a longitudinal study on very old individuals. Journal of Educational and Behavioural Statistics, 29(4), 421-437. 
Gilbert JJ, Simpson TL (1976) Sex reversal in a freshwater sponge. Journal of Experimental Zoology 195(1), 145-151.

Glynn PW (1988) El Niño-Southern Oscillation 1982-1983: Nearshore population, community and ecosystem responses. Annual Review of Ecology and Systematics, 19, 309-335.

Gómez A, Serra M, Carvalho GR, Lunt DH (2002) Speciation in ancient cryptic species complexes: evidence from the molecular phylogeny of Branchionus plicatilis (Rotifera). Evolution, 56, 1431-1444.

Goudet J (1995) FSTAT (version 1.2): a computer program to calculate F-statistics. Heredity, $86,485-486$.

Grapputo A, Kumpulainen T, Mappes J, Parri S (2005) Genetic diversity in populations of asexual and sexual bag worm moths (Lepidoptera: Psychidae). BMC Ecology, 29(5), 5.

Guardiola M, Frotscher J, Uriz MJ (2011) Genetic structure and differentiation at a short-time scale of the introduced calcarean sponge Paraleucilla magna to the western Mediterranean. In: Maldonado M, Turon X, Becerro M, Jesus Uriz M (Eds.) Ancient Animals, New Challenges Developments in Sponge Research, pp 71-84.

Guest JR, Baird AH, Goh BPL, Chou LM (2005) Seasonal reproduction in equatorial reef corals. Invertebrate Reproduction and Development, 48(1-3), 207-218.

Halenkoh U, Højsgaard S (2006) The R package geepack for generalized estimating equations. Journal of Statistical Software, 15(2), 1-11.

Hammel JU, Herzen J, Beckmann F, Nickel M (2009) Sponge budding is a spatiotemporal morphological patterning process: insights from synchrotron radiation-based $\mathrm{X}$-ray microtomography into the asexual reproduction of Tethya wilhelma. Frontiers in Zoology, 6, 19.

Harris TFW (1990) Greater Cook Straight form and flow. Levin, NZ: DSIR Marine and Freshwater.

Harrison HB, Berumen ML, Saenz-Agudelo P, Salas E, Williamson DH, Jones GP (2017) Widespread hybridization and bidirectional introgression in sympatric species of coral reef fish. Molecular Ecology, 26(20), 5692-5704.

Harrison RG, Larson EL (2014) Hybridization, introgression and the nature of species boundaries. Journal of Heredity, 105, 795-809.

Hatta M, Fukami H, Wang W, Omori M, Shimoike K, Hayashibara T, Ina Y, Sugiyama T (1999) Reproductive and genetic evidence for reticulate evolutionary history of mass-spawning corals. Molecular Biology and Evolution, 16(11), 1607-1613.

Hauser CL (1987) The debate about the biological species concept - a review. Journal of Zoological Systematics and Evolutionary Research, 25(4), 241-257. 
Haven ND (1971) Temporal patterns of sexual and asexual reproduction in the colonial ascidian Metandrocarpa taylori Hunstman. The Biological Bulletin, 140(3), 400-405.

Hayward A, Gilooly J (2011) The cost of sex: Quantifying energetic investment in gamete production by males and females. PloS One, 6(1), e16557.

Hayward BW, Grenfell HR, Sabaa AT, Carter R, Cochran U, Lipps JH, Shane PR, Morley MS (2006) Micropaleontological evidence of large earthquakes in the past 7200 years in southern Hawke's Bay, New Zealand. Quaternary Science Reviews, 25(11-12), 1186-1207.

Heath RA (1971) Hydrology and circulation in central and southern Cook Strait, New Zealand. New Zealand Journal of Marine and Freshwater Research, 5(1), 178-199.

Heath RA (1974). Sea level oscillations in Wellington Harbour. New Zealand Journal of Marine and Freshwater Research, 8(2), 403-414.

Heath RA (1981) Oceanic fronts around southern New Zealand. Deep Sea Research Part A. Oceanographic Research Papers, 28(6), 547-560.

Hebert PND, Penton EH, Burns JM, Janzen DH, Hallwachs W (2004) Ten species in one: DNA barcoding reveals cryptic species in the neotropical skipper butterfly Astraptes fulgerator. Proceedings of the National Academy of Sciences of the United States of America, 101(4), 14812-14817.

Heim I, Nickel M, Brümmer F (2007) Phylogeny of the genus Tethya (Tethyidae: Hadromerida: Porifera): molecular and morphological aspects. Journal of the Marine Biological Association of the United Kingdom, 87, 1615-1627.

Heiser CB (1949) Natural hybridization with particular reference to introgression. The Botanical Review, 15(10), 645-687.

Heiser CB (1973) Introgression re-examined. The Botanical Review, 39(4), 347-366.

Hellberg ME, Prada C, Tan MH, Forsman ZH, Baums IB (2016) Getting a grip at the edge: recolonization and introgression in eastern Pacific Porites corals. Journal of Biogeography, 43, 2147-2159.

Hennig W (1966) Phylogenetic Systematics. University of Illinois Press: Urbana.

Henry LA, Hart M (2005) Regeneration from injury and resource allocation in sponges and corals - a review. Hydrobiology 90(2), 125-158.

Hey J, Waples RS, Arnold ML, Butlin RK, Harrison RG (2003) Understanding and confronting species uncertainty in biology and conservation. Trends in Ecolgoy and Evolution, 18, 597603.

Heyward AJ, Negri AP (2010) Plasticity of larval pre-competency in response to temperature: observations on multiple broadcast spawning coral species. Coral Reefs, 29, 631-636. 
Hillis DM (1987) Molecular versus morphological approaches to systematics. Annual Review of Ecology, Evolution and Systematics, 18, 23-42.

Hill MS, Hill AL (2002) Morphological plasticity in the tropical sponge Anthosigmella varians: responses to predators and wave energy. The Biological Bulletin, 202(1), 86-95.

Hooper JNA (2003) 'Sponguide'. A guide to sponge collection and identification. In: Hooper JNA, Soest RWM Van (Eds.) 2002. Systema Porifera. A Guide to the Classification of Sponges, Kluwer Academic/Plenum Publishers: New York, Boston, Dordrecht, London, Moscow. Vol 1-2, pp xlviii-1708.

Hooper JNA, Van Soest RWM (2002) Systema Porifera. A Guide to the classification of sponges. Kluwer Academic/Plenum Publishers: New York, Boston, Dordrecht, London, Moscow.

Hooper JNA, Wiedenmayer F (1994) Porifera. In: Wells A (Ed.) Zoological Catalogue of Australia. CSIRO: Melbourne. Vol 12, pp 1-620.

Hoppe WF (1988) Reproductive patterns in three species of large coral reef sponges. Coral Reefs, 7, 45-50.

Hoppe WF, Reichert MJM (1987) Predictable annual mass release of gametes by the coral reef sponge Neofibularia nolitangere (Porifera: Demospongiae). Marine Biology, 94(2), 277-285.

Hsiung KM, Kimura S, Han YS, Takeshige A, lizuka Y (2018) Effect of ENSO events on larval and juvenile duration and transport of Japanese eel (Anguilla japonica). PLoS One, 13(4), e0195544.

Hulce D, Li X, Snyder-Leiby T (2011) GeneMarker genotyping software: Tools to increase the statistical power of DNA fragment analysis. Journal of Biomolecular Techniques, 22, S35-36.

Ilan M, Loya Y (1990) Sexual reproduction and settlement of the coral reef sponge Chalinula sp. from the Red Sea. Marine Biology, 105, 25-31.

Infante D, Gonzalez G, Peraza-Echeverria L, Keb-Llanes M (2003) Asexual genetic variability in Agave fourcroydes. Plant Science, 164(2), 223-230.

Inouye DW (2008) Effects of climate change on phenology, frost damage and floral abundance of Montane wildflowers. Ecology, 89(2), 352-362.

IPCC (2014) Climate change 2014: synthesis report. In: Core Writing Team, Pachauri RK, Meyer LA (Eds.) Contribution of Working Groups I, II and II to the Fifth Assessment Report of the Intergovernmental Panel on Climate Change. IPCC: Geneva, Switzerland, pp 151.

Ishii H, Ohba T, Kobayashi T (2008) Effects of low dissolved oxygen on planula settlement, polyp growth and asexual reproduction of Aurelia aurita. Plankton and Benthos Research, 3, 107-113.

Jackson JBC, Coates AG (1986) Life cycles and evolution of clonal (modular) animals. Philosophical Transactions of the Royal Society London B, 313(1159), 7-22. 
Jakobsson M, Rosenberg NA (2007) CLUMPP: a cluster matching ad permutation program for dealing with label switching and multimodality in analysis of population structure. Bioinformatics, 23(14), 1801-1806.

Johnson MF (1978) Studies on the reproductive cycles of the calcareous sponges Clathrina coriacea and C. blanca. Marine Biology, 50(1), 73-79.

Jokiel PL, Hildemann WH, Bigger CH (1982) Frequency of intercolony graft acceptance or rejection as a measure of population structure in the sponge Callyspongia diffusa. Marine Biology, 71(2), 135-139.

Jombart T (2008) Adegenet: R package for the multivariate analysis of genetic markers. Bioinformatics, 24, 1403-1405.

Jombart T, Devillard S, Balloux F (2010) Discriminant analysis of principal components: a new method for the analysis of genetically structured populations. BMC Genetics 11, 94.

Jones AG, Small CM, Paczolt KA, Ratterman NL (2010) A practical guide to methods of parentage analysis. Molecular Ecology Resources, 10, 6-30.

Kaplan EL, Meier P (1958) Nonparametric estimation from incomplete observations. Journal of the American Statistical Association, 53(282), 457-481.

Karako S, Achituv Y, Perl-Treves R, Katcoff D (2002) Asterina burtoni (Asteroidea: Echinodermata) in the Mediterranean and the Red Sea: Does asexual reproduction facilitate colonization? Marine Ecology Progress Series, 234, 139-145.

Karlson RH (1986) Disturbance, colonial fragmentation, and size-dependent life history variation in two coral reef cnidarians. Marine Ecology Progress Series, 28, 245-249.

Kauserud H, Svegarden IB, Decock C, Hallenberg N (2006) Hybridization among cryptic species of the cellar fungus Coniophora puteana (Basidiomycota). Molecular Ecology, 16, 389-399.

Kearse M, Moir R, Wilson A, Stones-Havas S, Cheung M, Sturrock S, Buxton S, Cooper A, Markowitz S, Duran C, Thierer T, Ashton B, Meintjes P, Drummond A (2012) Geneious Basic: an integrated and extendable desktop software platform for the organization and analysis of sequence data. Bioinformatics, 28, 1647-1649.

Keller LF, Waller DM (2002) Inbreeding effects in wild populations. Trends in Ecology and Evolution, 17(5), 230-241.

Kelly M, Herr B (2015) Splendid sponges: A guide to the sponges of New Zealand. National Institute of Water and Atmospheric Research, Ltd. New Zealand.

Kelly-Borges M, Pomponi SA (1992) The simple fool's guide to sponge taxonomy. Harbor Branch Oceanographic Institution. 
Kettenring KM, McCormick MK, Baron HM, Whigham DF (2011) Mechanisms in Phragmites australis invasion: feedbacks among genetic diversity, nutrients and sexual reproduction. Journal of Applied Ecology, 48(5), 1305-1313.

Klautau M, Russo CAM, Lazoski C, Boury-Esnault N, Thorpe JP, Sole-Cava AM (1999) Does cosmopolitanism result from overconservative systematics? A case study using the marine sponge Chonrilla nucula. Evolution, 53(5), 1414-1422.

Knapp IS, Forsman ZH, Williams GJ, Toonen RJ, Bell JJ (2015) Cryptic species obscure introduction pathway of the blue Carribean sponge (Haliclona (Soestella) caerulea), (order: Haplosclerida) to Palmyra Atoll, Central Pacific. PeerJ, 3, e1170.

Knowlton N (1993) Sibling species in the sea. Annual Review of Ecology and Systematics, 24(1), 189-216.

Knowlton N (2000) Molecular genetic analysis of species boundaries in the sea. Hydrobiologia, 420, 73-90.

Knowlton N, Maté JL, Guzmàn HM, Rowan R, Jara J (1997) Direct evidence for reproductive isolation among the three species of the Monastraea annularis complex in Central America (Panama and Honduras). Marine Biology, 127(4), 705-711.

Krishnamurthy PK, Francis RA (2012) A critical review on the utility of DNA barcoding in biodiversity conservation. Biodiversity and Conservation, 21(8), 1901-1919.

Kroeker KJ, Kordas RL, Crim RN, Singh GG (2010) Meta-analysis reveals negative yet variable effects of ocean acidification on marine organisms. Ecology Letters, 13, 1419-1434.

Krueger J (2001) Null hypothesis significance testing: On the survival of a flawed method. American Psychologist, 56(1), 16-26.

Kuklinski P, Berge J, McFadden L, Dmoch K, Zajaczkowski M, Nygard H, Piwosz K, Tatarek A (2013) Seasonality of occurrence and recruitment of Arctic marine benthic invertebrate larvae in relation to environmental variables. Polar Biology, 36(4), 549-560.

Kumar S, Stecher G, Tamura K (2015) MEGA7: Molecular Evolutionary Genetics Analysis version 7.0 for bigger datasets. Molecular Biology and Evolution, 33, 1870-1874.

Kurelec B, Pivcevic B (1992) The multidrug resistance-like mechanism in the marine sponge Tethya aurantium. Marine Environmental Research, 34(1-4), 249-253.

Ladner JT, Palumbi SR (2012) Extensive sympatry, cryptic diversity and introgression throughout the geographic distribution of two coral species complexes. Molecular Ecology, 21(9), 2224-2238.

Lande R (1980) Genetic variation and phenotypic evolution during allopatric speciation. The American Naturalist, 116(4), 462-479.

Lande R, Schemske DW (1985) The evolution of self-fertilization and inbreeding depression in plants - I. genetic models. Evolution, 39(1), 24-40. 
Lande R, Shannon S (1996) The role of genetic variation in adaptation and population persistence in a changing environment. Evolution, 50(1), 434-437.

Lanna E, Paranhos R, Paiva PC, Klautau M (2014) Environmental effects on the reproduction and fecundity of the introduced calcareous sponge Paraleucilla magna in Rio de Janeiro, Brazil. Marine Ecology, 36, 1075-11087.

Larkin MA, Blackshields G, Brown NP, Chenna R, McGettigan PA, McWilliam H, Valentin F, Wallace IM, Wilm A, Lopez R, Thompson JD, Gibson TJ, Higgins DG (2007) ClustalW and ClustalX. Bioinformatics, 23, 2947-2948.

Lavrov DV, Wang XJ, Kelly M (2008) Reconstructing ordinal relationships in the Demospongiae using mitochondrial genomic data. Molecular Phylogenetics and Evoltuion, 49, 111-124.

Law CS, Rickard GJ, Mikaloff-Fletcher SE, Pinkerton MH, Behrens E, Chiswell SM, Currie K (2017) Climate change projections for the surface ocean around New Zealand. New Zealand Journal of Marine and Freshwater Research, DOI: 10.1080/00288330.2017.1390772.

Lawrence AJ, Soame JM (2004) The effects of climate change on the reproduction of coastal invertebrates. International Journal of Avian Science, 146(s1), 29-39.

Lazoski C, Sole-Cava A, Boury-Esnault N, Klautau M, Russo CAM (2001) Cryptic speciation in a high gene flow scenario in the oviparous marine sponge Chondrosia reniformis. Marine Biology, 139(2), 421-429.

Lecompte E, Denys C, Granjon L (2005) Confrontation of morphological and molecular data: The Praomys group (Rodentia, Murinae) as a case of adaptive convergences and morphological stasis. Molecular Phylogenetics and Evolution, 37, 899-919.

Ledenfield R (1888) Descriptive catalogue of sponges in the Australian Museum, Sydney. Taylor and Francis: London.

Lee HW, Ban S, Ikeda T, Matsuishi T (2003) Effect of temperature on development, growth and reproduction in the marine copepod Pseudocalanus newmani at satiating food condition. Journal of Plankton Research 25(3), 261-271.

Lehtonen J, Jennions MD, Kokko M (2012) The many costs of sex. Trends in Ecology and Evolution, 27(3), 172-178.

Leong W, Pawlik JR (2011) Comparison of reproductive patterns among 7 Caribbean sponge species does not reveal a resource trade-off with chemical defenses. Journal of Experimental Marine Biology and Ecology, 401, 80-84.

Leys SP, Yahel G, Reidenbach MA, Tunnicliffe V, Shavit U, Reiswig HM (2011) The sponge pump: the role of current induced flow in the design on the sponge body plan. PLoS One, 6(12), e27787.

Li A, Ge S (2001) Genetic variation and clonal diversity of Psammochloa villosa (Poaceae) detected by IISR markers. Annals of Botany, 87(5), 595-590. 
Li CW, Chen JY, Hua TE (1998) Precambrian sponges with cellular structure. Science, 5352, 879-882.

Li Y, Huang JK, Li H, Liu J, Yang W (2016) Genetic diversity of Ulva prolifera population in Qingdao coastal waters during the green algal blooms revealed by microsatellite. Marine Pollution Bulletin, 111(1-2), 237-246.

Lihová J, Kučera J, Perný M, Marhold K (2007) Hybridization between two polypoloid Cardamine (Brassicacea) species in north-western Spain: Discordance between morphological and genetic variation patterns. Annals of Botany, 99, 1083-1096.

Littlefield CL, Finkemeier C, Bode HR (1991) Spermatogenesis in Hydra oligactis: II. How temperature controls the reciprocity of sexual and asexual reproduction. Developmental Biology, 146(2), 292-300.

López-Legentil S, Pawlik JR (2008) Genetic structure of the Caribbean giant barrel sponge Xestospongia muta using the I3-M11 partition of COI. Coral Reefs, 28(1), 157-165.

Lorenzen CJ (1970) Surface chlorophyll as an index of the depth, chlorophyll content, and primary productivity of the euphotic zone. Limnology and Oceanography, 15, 479-480.

Lowe WH, Allendorf FW (2010) What can genetics tell us about population connectivity? Molecular Ecology, 19, 3038-3051.

Lubzens E, Minkoff G, Marom S (1985) Salinity dependence of sexual and asexual reproduction in the rotifer Brachionus plicatilis. Marine Biology, 85(2), 123-126.

Maldonado M (1998) Do chimeric sponges have improved chances of survival? Marine Ecology Progress Series, 154:301-306.

Maldonado M (2006) The ecology of sponge larva. Canadian Journal of Zoology, 84, 175-194.

Maldonado M, Bergquist PR (2002) Phylum porifera. In Young, C.M. (Ed.) Atlas of Marine Invertebrate Larvae (pp 21-50). London: Academic Press.

Maldonado M, Riesgo A (2008) Reproduction in the phylum Porifera: a synoptic overview. Treballs de la Societat Catalana de Biologia, 59, 29-49.

Maldonado M, Uriz MJ (1999) Sexual propagation by sponge fragments. Nature, 398, 476.

Mariani S, Piscitelli MP, Uriz MJ (2001) Temporal and spatial co-occurrence in spawning and larval release of Cliona viridis (Porifera: Hadromerida). Journal of the Marine Biological Association of the United Kingdom, 81, 565-567.

Mariani S, Uriz MJ, Turon X, Alcoverro T (2006) Dispersal strategies in sponge larvae: integrating the life history of larvae and the hydrologic component. Oecologia, 149, 174-184.

Marriage TN, Kelly JK (2009) Inbreeding depression in an asexual population of Mimulus guttatus. Journal of Evolutionary Biology, 22(11), 2320-2331. 
Mayden RL (1997) A hierarchy of species concepts: The denouement in the saga of the species problem. In: Claridge MF, Dawah HA, Wilson MR (Eds.) Species: The Units of Biodiversity, Chapman and Hall: London, pp 381-424.

Maynard Smith J (1971) The origin and maintenance of sex. In: Williams, GC (Ed.) Group Selection, Aldine, Chicago, pp 163-175.

Maynard Smith J (1978) The evolution of sex. Cambridge, UK: Cambridge University.

Mayr E (1942) Systematics and the origin of species. Columbia University Press: New York.

McBride RS, Somarakis S, Fitzhugh GR, Albert A, Yaragina NA, Wuenschel MJ, AlonsoFernandez A, Basilone G (2013) Energy acquisition and allocation to egg production in relation to fish reproductive strategies. Fish and Fisheries, 16(1), 23-57.

McCormack GP, Erpenbeck D, Van Soest RWM (2002) Major discrepancy between phylogenetic hypotheses based on molecular and morphological criteria within the Order Haplosclerida (Phylum Porfera: Class Demospongiae). Journal of Zoological Sytematics and Evolutionary Research, 40, 237-240.

McDonald JI, Hooper JNA, McGuinness KA (2002) Environmentally influenced variability in the morphology of Cinachyrella australiensis (Carter 1886) (Porifera: Spirophorida: Tellidae). Marine and Freshwater Research, 53, 79-84.

McFadden, CS (1997) Contribution of sexual and asexual reproduction to population structure in the clonal soft coral, Alcyonium rudyi. Evolution, 51(1), 112-126.

McGhee KE (2006) The importance of life-history stage and individual variation in the allorecgonition system of a marine sponge. Journal of Experimental Marine Biology and Ecology 333(2), 241-250.

Mcgovern TM, Hellberg ME (2003) Cryptic species, cryptic endosymbionts, and geographical variation in chemical defences in the bryozoan Bugula neritina. Molecular Ecology, 12, 12071215.

Meglécz E, Costedoat C, Dubut V, Gilles A, Malausa T, Pech N, Martin JF (2009) QDD: a user-friendly program to select microsatellite markers and design primers from large sequencing projects. Bioinformatics, 26(3), 403-404.

Mendes JM, Woodley JD (2002) Timing of reproduction in Montastraea annularis: relationship to environmental variables. Marine Ecology Progress Series, 227, 241-251.

Mercado-Molina AE, Sabat AM, Yoshioka PM (2011) Demography of the demosponge Amphimedon compressa: evaluation of the importance of sexual versus asexual recruitment to its population dynamics. Journal of Experimental Marine Biology and Ecology, 407(2), 355362.

Mercier A, Sun Z, Baillon S (2011) Lunar rhythms in the deep sea: evidence from the reproductive periodicity of several marine invertebrates. Journal of Biological Rhythms, 26(1), 82-86. 
Mercurio M, Corriero G, Gaino E (2007) A 3-year investigation of sexual reproduction in Geodia cydonium (Jameson 1811) (Porifera, Demospongiae) from a semi-enclosed Mediterranean bay. Marine Biology, 151, 1491-1500.

Meyer CP, Paulay G (2005) DNA barcoding: error rates based on comprehensive sampling. PLoS Biology, 3(12), e422.

Miller K, Alvarez B, Battershill C, Northcote P, Parthasarathy H (2001) Genetic, morphological, and chemical divergence in the sponge genus Latruncuila (Porifera: Demospongiae) from New Zealand. Marine Biology, 139(2), 235-250.

Minchin D (1992) Multiple species, mass spawning events in an Irish sea lough: the effect of temperature on spawning and recruitment of invertebrates. Invertebrate Reproduction and Development, 22(1-3), 229-238.

Morrow C, Cárdenas P (2015) Proposal for a revised classification of the Demospongiae (Porifera). Frontiers in Zoology, 12, 7.

Mortiz C, Cicero C (2004) DNA barcoding: promise and pitfalls. PLoS Biology, 2(10), e354.

Muirhead CA, Lande R (1997) Inbreeding depression under joint selfing, outcrossing and asexuality. Evolution, 51(5), 1409-1415.

Muller HJ (1932) Some genetic aspects of sex. The American Naturalist, 66(703), 118-138.

Mundry R (2011) Issues in information theory-based statistical inference - a commentary from frequentist's perspective. Behavioral Ecology Sociobiology, 65, 57-68.

Muricy G, Solé-Cava AM, Thorpe JP, Boury-Esnault N (1996) Genetic evidence for extensive cryptic speciation in the subtidal sponge Plakina trilopha (Porifera: Demospongiae: Homoscleromorpha) from the Western Mediterranean. Marine Ecology Progress Series, 138, 181-187.

Murphy RJ, Pinkerton MH, Richardson KM, Bradford-Grieve JM, Boyd PW (2001) Phytoplankton distributions around New Zealand derived from SeaWiFS remotely-sensed ocean colour data. New Zealand Journal of Marine and Freshwater Research, 35(2), 343-362.

Nakayama S, Arima K, Kawai K, Mohri K, Inui C, Sugano W, Koba H, Tamada K, Nakata YJ, Kishimoto K, Arai-Shindo M, Kojima C, Matsumoto T, Fujimori T, Agata K, Funayama N (2015) Dynamic transport and cementation of skeletal elements build up the pole-and-beam structured skeleton of sponges. Current Biology, 25, 2549-2554.

Naylor E (2013) Rhythmic behaviour and reproduction in marine animals. In: Newell RC (Ed.) Adaptation to Environment: Essays on the Physiology of Marine Animals. Elsevier. pp 393430.

Negri AP, Marshall PA, Heyward AJ (2007) Differing effects of thermal stress on coral fertilization and early embryogenesis in four Indo Pacific species. Coral Reefs, 26(4), 759-763. 
Neigel JE, Avise JC (1983) Histocompatibility bioassays of population structure in marine sponges: Clonal structure in Verongia longissimi and Iotrochota birotulata. Journal of Heredity, 74(3), 134-140.

Nichols SA, Barnes PAG (2005) A molecular phylogeny and historical biogeography of the marine sponge genus Placospongia (Phylum Porifera) indicate low dispersal capabilities and widespread crypsis. Journal of Experimental Marine Biology and Ecology, 323(1), 1-15.

Nickel M (2004) Kinetics and rhythm of body contractions in the sponge Tethya wilhelma (Porifera: Demospongiae). Journal of Experimental Biology, 26, 4515-4524.

Niemiller ML, Fitzpatrick BM, Miller BT (2008) Recent divergence with gene flow in Tennessee cave salamanders (Plethodontidae: Gyrinophilus) inferred from gene genealogies. Molecular Ecology, 17(9), 2258-2275.

Nosil P (2008) Speciation with gene flow could be common. Molecular Ecology, 17(9), 21032106.

Nozawa Y, Huang YS, Hirose E (2016) Seasonality and lunar periodicity in the sexual reproduction of the coral-killing sponge, Terpios hoshinota. Coral Reefs, 35, 1071-1081.

Nydam ML, Harrison RG (2010) Introgression despite substantial divergence in a broadcast spawning marine invertebrate. Evolution, 65(2), 429-442.

Nylander JAA (2004) MrModeltest v2. Program distributed by the author. Evolutionary Biology Centre, Uppsala University.

O’Dea A, Okamura B (1999) Influence of seasonal variation in temperature, salinity and food availability on module size and colony growth of the estuarine bryozoan Conopeum seurati. Marine Biology, 135(4), 581-588.

Olive PJW (2011) The adaptive significance of seasonal reproduction in marine invertebrates: the importance in distinguishing between modes. Invertebrate Reproduction and Development, $22,1-3$.

Olive PJ, Rees SW, Djunaedi A (1998) Influence of photoperiod and temperature on oocyte growth in the semelparous polychaete Nereis (Neanthes) virens. Marine Ecology Progress Series, 172, 169-183.

Oliver JK, Babcock RC, Harrison PL, Willis BL (1988) Geographic extent of mass coral spawning: clues to ultimate causal factors. Proceedings of the 6th International Coral Reef Symposium, 2, 853-859.

Padial JM, Miralles A, De la Riva I, Vences M (2010) The integrative future of taxonomy. Frontiers in Zoology, 7, 16.

Paixão L, Ferreira MA, Nunes Z, Fonseca-Sizo F, Rocha R (2013) Effects of salinity and rainfall on the reproductive biology of the mangrove oyster (Crassostra gasar): Implications for the collection of broodstock oysters. Aquaculture, 308-383, 6-12. 
Palumbi SR (1994) Genetic divergence, reproductive isolation and marine speciation. Annual Review of Ecology and Systematics, 25, 547-572.

Palumbi SR (2003) Population genetics, demographic connectivity, and the design of marine reserves. Ecological Applications, 13(1), 146-158.

Pante E, Puilandre N, Viricel A, Arnaud-Haond S, Aurelle D, Castelin M, Chenuil A, Destombe C, Forcioli D, Valero M, Viard F, Samadi S (2015) Species are hypotheses: avoid connectivity assessments based on pillars of sand. Molecular Ecology, 24(3), 525-544.

Paris CB, Cherubin LM, Cowen RK (2007) Surfing, spinning or diving from reef to reef: effects on population connectivity. Marine Ecology Progress Series, 347, 285-300.

Parmesan C, Yohe G (2003) A globally coherent fingerprint of climate change impacts across natural systems. Nature, 421, 37-42.

Peakall R, Smouse PE (2012) GenAlEx 6.5: genetic analysis in Excel. Population genetic software for teaching and research-an update. Bioinformatics, 28, 2537-2539.

Perea-Blàzquez A (2011) Interactions between sponges and the water column: nutrient utilisation and feeding by New Zealand subtidal sponges. PhD Thesis, Victoria University of Wellington, Wellington, New Zealand. URI: http://hdl.handle.net/10063/1866.

Perez-Portela R, Noyer C, Becerro MA (2014) Genetic structure and diversity of the endangered bath sponge Spongia lamella. Aquatic Conservation, 25(3), 365-379.

Pfeiffer T, Klahr A, Peterson A, Levichev IG, Schnittler M (2012) No sex at all? Extremely low genetic diversity in Gagea spathacea (Lilaceae) across Europe. Flora - Morphology, Distribution, Functional Ecology of Plants, 207(5), 372-378.

Pickrill RA, Mitchell JS (1979) Ocean wave characteristics around New Zealand. New Zealand Journal of Marine and Freshwater Research, 13(4), 501-520.

Pineda J, Hare JA, Sponaugle S (2007) Larval transport and dispersal in the coastal ocean and consequences for population connectivity. Oceanography, 20(3), 22-39.

Plotkin A, Voight O, Willassen E, Rapp HT (2017) Molecular phylogenies challenge the classification of Polymastiidae (Porifera, Demospongiae) based on morphology. Organisms Diversity and Evolution, 17, 45-66.

Pomeroy LR (1991) Relationships of primary and secondary production in lakes and marine ecosystems. Comparative Analyses of Ecosystems, 97-119.

Pond D, Harris R, Head R, Harbour D (1996) Environmental and nutritional factors determining seasonal variability in the fecundity and egg viability of Calanus helgolandicus in coastal waters off Plymouth, UK. Marine Ecology Progress Series 143, 45-63.

Poole MA, O'Farrell PN (1971) The assumptions of the linear regression model. Transactions of the Institute of British Geographers, 52, 145-158. 
Poppe J, Sutcliffe P, Hooper JNA, Wörheide G, Erpenbeck D (2010) COI barcoding reveals new clades and radiation patterns of Indo-Pacific sponges of the family Irciniidae (Demospongiae: Dictyoceratida). PLoS One, 5, e9950.

Prentis PJ, Wilson JRU, Dormontt EE, Richardson DM, Lowe AJ (2008) Adaptive evolution in invasive species. Trends in Plant Science 13(6), 288-294.

Pritchard JK, Stephens M, Donnelly P (2000) Inference of population structure using multilocus genotype data. Genetics, 155(2), 945-959.

Pritchard K, Ward V, Battershill C, Bergquist PR (1984) Marine sponges: Forty-six sponges of Northern New Zealand. Leigh Laboratory Bulletin, 14, 149.

Procaccini G, Mazella L (1998) Population genetic structure and gene flow in the seagrass Posidonia oceanica assessed using microsatellite analysis. Marine Ecology Progress Series, 169, 133-141.

Przeslawski R, Ahyong S, Byrne M, Wörheide G, Hutchings P (2008) Beyond corals and fish: the effects of climate change on noncoral benthic invertebrates of tropical reefs. Global Change Biology, 14, 2773-2795.

Puill-Stephan E, Willis BL, van Herwerden L, van Oppen MJ (2009) Chimerism in wild adult populations of the broadcast spawning coral Acropora millepora on the Great Barrier Reef. PLoS One, 4(11), e7751.

Purcell JE (2007) Environmental effects on asexual reproduction rates of the scyphozoan Aurelia labiate. Marine Ecology Progress Series, 348, 183-196.

Purcell JE, White JR, Nemazie DA, Wright DA (1999) Temperature, salinity and food effects on asexual reproduction and abundance on the scyphozoan Chrysaora quinquecirrha. Marine Ecology Progress Series, 180, 187-196.

R Core Team (2017) R: A language and environment for statistical computing. R Foundation for Statistical Computing, Vienna, Austria. URL: https://www.R-project.org/.

Rasheed MA (2004) Recovery and succession in a multi-species tropical seagrass meadow following experimental disturbance: the role of sexual and asexual reproduction. Journal of Experimental Marine Biology and Ecology, 310(1), 13-45.

Rasmont R (1962) The physiology of gemmulation in fresh-water sponges. Symposium of the Society for the Study of Development and Growth, 20, 3-25.

Raymond M, Rousset F (1995) GENEPOP (version 1.2): population genetics software for exact tests and ecumenicism. Journal of Heredity, 86, 248-249.

Redmond NE, McCormack GP (2009) Ribosomal internal transcriber spacer regions are not suitable for intra- or inter-specific phylogeny reconstruction in haplosclerid sponges (Porifera: Demospongiae). Journal of the Marine Biological Association of the UK, 89, 1251-1256. 
Rieseberg LH (1998) Molecular ecology of hybridization. In: Carvallo GR (Ed.) Advances in Molecular Ecology, IOS Press.

Reiswig HM (1973) Population dynamics of three Jamaican demospongiae. Bulletin of Marine Science, 23, 191-226.

Reiswig HM (1975) Bacteria as food for temperate-water marine sponges. Canadian Journal of Zoology, 53(5), 582-589.

Reveillaud J, Remerie T, Van Soest R, Erpenbeck D, Cardenas P, Derycke S, Xavier JR, Rigaux A, Vanreusal A (2010) Species boundaries and phylogenetic relationships between Atlanto-Mediterranean shallow-water and deep-sea coral associated with Hexadella species (Porifera, Ianthellidae). Molecular Phylogenetics and Evolution, 56, 104-114.

Reveillaud J, van Soest R, Derycke S, Picton B, Rigaux A, Vanreusel A (2011) Phylogenetic relationships among NE Atlantic Plocamionida Topsent (1927) (Porifera, Poecilosclerida): under-estimated diversity in reef ecosystems. PLoS One, 6, e16533.

Rhymer JM, Simberloff D (1996) Extinction by hybridization and introgression. Annual Review of Ecology and Systematics, 27, 83-109.

Rice WR (1989) Analyzing tables of statistical tests. Evolution, 43, 223-235.

Richmond GH (1990) The effects of the El Niño/Southern Oscillation on the dispersal or corals and other marine organisms. Elsevier Oceanography Series, 52, 127-140.

Rideout RS (1978) Asexual reproduction as a means of populations maintenance in the coral reef asteroid Linckia multifora in Guam. Marine Biology, 47, 287-295.

Riesgo A, Maldonado M (2008) Differences in reproductive timing among sponges sharing habitat and thermal regime. Invertebrate Biology, 127(4), 357-367.

Riesgo A, Maldonado M, Durfort M (2007) Dynamics of gametogenesis, embryogenesis, and larval release in a Mediterranean homosclerophorid demosponge. Marine and Freshwater Research, 58(4), 398-417.

Riesgo A, Pérez-Portela R, Pita L, Blasco G, Erwim PM, López-Legentil S (2016) Population structure and connectivity in the Mediterranean sponge Ircinia fasciculata are affected by mass mortalities and hybridization. Heredity, 117, 427-439.

Roberts CM (1997) Connectivity and management of Caribbean coral reef. Science, 278(5342), 1454-1457.

Roberts DE, Davis AR, Cummins SP (2006) Experimental manipulation of shade, silt, nutrients and salinity on the temperate reef sponge Cymbastela concentrica. Marine Ecology Progress Series, 307, 143-154.

Rodrigues N, Sharma R, Nath BN (2001) Impact of benthic disturbance on megafauna in Central Indian Basin. Deep Sea Research II: Topical Studies in Oceanography, 48(16), 34113426. 
Ronquist, F, Teslenko M, Van der Mark P, Ayres D, Darling A, Höhna S, Larget B, Lui L, Suchard MA, Huelsenbeck JP (2012) MrBayes 3.2: Efficient Bayesian phylogenetic inference and model choice across a large model space. Systematic Biology, 61, 539-542.

Roques S, Sevigny JM, Bernatchez L (2001) Evidence for broadscale introgressive hybridization between two redfish (genus Sebastes) in the North-west Atlantic: a rare marine example. Molecular Ecology, 10(1), 149-165.

Rosen DE (1979) Fishes from the uplands and intermontane basins of Guatemala: Revisionary studies and comparative geography. Bulletin of the American Museum of Natural History, 162, 267-376.

Ross PM, Hogg ID, Pilditch CA, Lundquist CJ (2009) Phylogeography of New Zealand's coastal benthos. New Zealand Journal of Marine and Freshwater Research, 43(5), 1009-1027.

Rot C, Goldfarb I, Ilan M, Huchon D (2006) Putative cross-kingdom horizontal gene transfer in sponge (Porifera) mitochondria. BMC Evolutionary Biology, 6, 71.

Roux C, Tsagkogeorga G, Bierne N, Galtier N (2013) Crossing the species barrier: genomic hotspots of introgression between two highly divergent Ciona intestinalis species. Molecular Biology and Evolution, 30(7), 1574-1587.

Roze D (2012) Disentangling the benefits of sex. PLoS Biology, 10(5), e1001321.

Rüber L, Meyer A, Sturmbauer C, Verheyen E (2001) Population structure in two sympatric species of the Lake Tanganyika cichlid tribe Eretmondini: evidence for introgression. Molecular Ecology, 10, 1207-1225.

Rundell RJ, Price TD (2009) Adaptive radiation, nonadaptive radiation, ecological speciation and nonecological speciation. Trends in Ecology and Evolution, 24(7), 394-399.

Russo CAM, Sole-Cava AM, Thorpe JP (1994) Population structure and genetic variation in two tropical sea anemones (Cnidaria, Actinidae) with different reproductive strategies. Marine Biology, 119(2), 267-276.

Ryan WH (2018) Temperature-dependent growth and fission rate plasticity drive seasonal and geographic changes in body size in a clonal sea anemone. The American Naturalist, 191(2), 210-219.

Sara M (1974) Sexuality in the Porifera. Italian Journal of Zoology 41(4), 327-348.

Sarà M (1984) Reproductive strategies in sessile macrofauna. Italian Journal of Zoology, 51, 243-248.

Sarà M (1987) A Study on the Genus Tethya (Porifera Demospongiae) and New Perspectives in Sponge Systematics. In: Vacelet J, Boury-Esnault N (Eds.) Taxonomy of Porifera. NATO ASI Series (Series G: Ecological Sciences), Springer: Berlin, Vol 13. 
Sarà M (1998) A biogeographic and evolutionary survey of the genus Tethya (Porifera, Demospongiae). In: Watanabe Y, Fusetani N (Eds.). Sponge Sciences, Springer: Tokyo, Vol 1, pp. 83-94.

Sarà M, Bavestrello G (1995) Tethya omanensis, a remarkable new species from an Oman cave (Porifera, Demospongiae). Bolletino di Zoologia, 62(1), 23-27.

Sarà M, Corriero G, Bavestrello G (1993) Tethya (Porifera, Demospongiae) species coexisting in a Maldivian coral reef lagoon: Taxonomical, genetic and ecological data. Marine Ecology, $14,341-355$.

Sarà M, Sarà A (2004) A revision of Australian and New Zealand Tethya (Porifera: Demospongiae) with a preliminary analysis of species-groupings. Invertebrate Systematics, 18(2), 117-156.

Sastry AN (1966) Temperature effects in reproduction of the bay scallop Aequipecten irradians Lamarck. The Biological Bulletin, 130(1), 118-134.

Schneider CA, Rasband WS, Eliceiri KW (2012) NIH Image to ImageJ: 25 years of image analysis. Nature Methods, 9, 671-675.

Sebens KP (1979) The energetics of asexual reproduction and colony formation in benthic marine invertebrates. American Zoology, 19(3), 683-687.

Sebens KP (1980) The regulation of asexual reproduction and indeterminate body size in the sea anemone Anthopleura elegantissima (Brandt). The Biological Bulletin, 158(3), 370-382.

Shearer TL, Coffroth MA (2008) DNA barcoding: Barcoding corals: limited by interspecific divergence, not intraspecific variation. Molecular Ecology Resources, 8, 247-255.

Shears NT, Bowen MM (2017) Half a century of coastal temperature records reveal complex warming trends in western boundary currents. Scientific Reports, 7, 14527.

Sherman CHD, Ayre DJ, Miller KJ (2006) Asexual reproduction does not produce clonal populations of the brooding coral Pocillopora damicornis on the Great Barrier Reef, Australia. Coral Reefs, 25(1), 7-18.

Sherry RA, Zhou X, Gu S, Arnone JA III, Schimel DS, Verburg PS, Wallace LL, Luo Y (2004) Divergence of reproductive phenology under climate warming. Proceedings of the National Academy of Science, 104(1), 198-202.

Shick JA, Lamb AN (1977) Asexual reproduction and genetic population structure in the colonizing sea anemone Haliplanella luciae. The Biological Bulletin, 153(3), 604-617.

Shreve SM, Mockford EL, Johnson KP (2011) Elevated genetic diversity of mitochondrial genes in asexual populations of Bark Lice ('Psocoptera': Echmepteryx hageni). Molecular Ecology, 20(21), 4433-4451. 
Silvertown J (2008) The evolutionary maintenance of sexual reproduction: Evidence from the ecological distribution of asexual reproduction in clonal plants. International Journal of Plant Science, 169(1), 157-68.

Simon JC, Rispe C, Sunnucks P (2002) Ecology and evolution of sex in aphids. Trends in Ecology and Evolution, 17(1), 34-39.

Simpson GG (1951) The species concept. Evolution, 5, 285-298.

Simpson TL (1980) Reproductive processes in sponges: a critical evaluation of current data and views. International Journal of Invertebrate Reproduction, 2(4), 251-269.

Simpson TL (2012) The cell biology of sponges. New York, USA: Springer-Verlag New York Inc.

Simpson TL, Fell PE (1974) Dormancy among the Porifera: Gemmule formation and germination in fresh-water and marine sponges. Transactions of the American Microscopical Society, 93(4), 544-577.

Simpson TL, Gilbert JJ (1973) Gemmulation, gemmule hatching, and sexual reproduction in fresh-water sponges I. Life cycle of Spongilla lacustris and Tubella pennsylvanica. Transactions of the American Microscopical Society, 92(3), 422-433.

Snell TW (1986) Effect of temperature, salinity and food level on sexual and asexual reproduction in Brachionus plicatlis (Rotifera). Marine Biology, 92(2), 157-162.

Solé-Cava AM, Boury-Esnault N (1999) Patterns of intra and interspecific genetic divergence in marine sponges. Memoirs of the Queensland Museum, 44, 591-601.

Solé-Cava AM, Klautau M, Boury-Esnault N, Borojecic R, Thorpe JP (1991) Genetic evidence for cryptic speciation in allopatric populations of two cosmopolitan species of the calcareous sponge genus Clathrina. Marine Biology, 111, 381-386.

Soliva M, Widmer A (2007) Gene flow across species boundaries in sympatric sexually deceptive Ophrys (Orchidaeae) species. Evolution, 57(10), 2252-2261.

Sommerfeldt AD, Bishop JDD, Wood CA (2003) Chimerism following fusion in a clonal ascidian (Urochordata). Biological Journal of Linnean Society, 79(1), 183-192.

Sponer R, Roy MS (2002) Phylogeographic analysis of the brooding brittle star Amphipholis squamata (Echinodermata) along the coast of New Zealand reveals high cryptic genetic variation and cryptic dispersal potential. Evolution, 56, 1954-1967.

Stenberg P, Lundmark M, Saura A (2003) MLGsim: a program for detecting clones using a simulation approach. Molecular Ecology Resources, 3(2), 329-331.

Stenøien HK (2008) Slow molecular evolution in 18S rDNA, rbcL and nad5 genes of mosses compared with higher plants. Journal of Evolutionary Biology, 21(2), 566-571. 
Stephens KM, Ereskovsky A, Lalor P, McCormack GP (2013) Ultrastructure of the ciliated cells of the free-swimming larva, and sessile stages, of the marine sponge Haliclona indistincta (Demospongiae: Haplosclerida). Journal of Morphology, 274, 1263-1276.

Stevens MI, Hoggs ID (2004) Population genetic structure of New Zealand's endemic corophiid amphipods: evidence for allopatric speciation. Biological Journal of Linnean Society, 81, 119-133.

Sun, Z, Hamel JF, Mercier A (2012) Marked shifts in offspring size elicited by frequent fusion among siblings in an internally brooding marine invertebrates. The American Naturalist, 180(5), E151-E160.

Swofford DL (2002) PAUP*. Phylogenetic Analysis Using Parsimony (*and Other Methods). Version 4. Sinaeur Associates, Sunderland, Massachusetts.

Tait A, Turner R (2005) Generating multiyear gridded daily rainfall over New Zealand. Journal of Applied Meteorology, 44(9), 1315-1323.

Tam JC (2012) Intertidal community differences between the Cook Strait and Wellington Harbour. PhD Thesis, Victoria University of Wellington, Wellington, New Zealand. HDL:10063/32.

Tanner JE (1996) Seasonality and lunar periodicity in the reproduction of Pocilloporid corals. Coral Reefs, 15(1), 59-66.

Tatarenkov A, Bergström L, Jönsson RB, Serrão EA, Kautsky L, Johannesson K (2005). Intriguing asexual life in marginal populations of the brown seaweed Fucus vesiculosus. Molecular Ecology, 14(2), 647-651.

Teixido N, Gili JM, Uriz MJ, Gutt J, Arntz WE (2006) Observations of asexual reproductive strategies in Antarctic hexactinellid sponges from ROV video records. Deep-sea Research II, 53, 972-984.

Thompson RJ (1983) The relationship between food ration and reproductive effort in the green sea urchin, Strongylocentrotus droebachiensis. Oecologia, 56(1), 50-57.

Thomsen E, Håkansson E (1995) Sexual versus asexual dispersal in clonal animals: examples from cheilostome bryozoans. Paleobiology, 21(4), 496-508.

Tökölti J, Bradács F, Hóka N, Kozma N, Miklós M, Mucza O, Lénárt K, Ősz Z, Sebestyén F, Barta Z (2016) Effects of food availability on asexual reproduction and stress tolerance along the fast-slow life history continuum in freshwater hydra (Cnidaria: Hydrozoa). Hydrobiologia, $766,121-133$.

Tooley PW, Sweigard JA, Fry WE (1986) Fitness and virulence of Phytophthora infestans isolates from sexual and asexual populations. Phytopathology, 76, 1209-1212.

Tourinho JL, Solé-Cava AM, Lazoski C (2012) Cryptic species within the commercially most important lobster in the tropical Atlantic, the spiny lobster Panulirus argus. Marine Biology, 159(9), 1897-1906. 
Treml EA, Roberts JJ, Halpin PN, Possingham HP, Riginos C (2012) Reproductive output and duration of the pelagic larval stage determine seascape-wide connectivity of marine populations. Integrative and Comparative Biology, 52(4), 525-537.

Triantafillos L, Adams M (2005) Genetic evidence that the northern calamari, Sepioteuthis lessoniana, is a species complex in Australian waters. ICES Journal of Marine Science, 62(8), 1665-1670.

Turon X, Tarjuelo I, Uriz MJ (2002). Growth dynamics and mortality of the encrusting sponge Crambe crambe (Poecilosclerida) in contrasting habitats: correlation with population structure and investment in defence. Functional Ecology, 12(4), 631-639.

Uecker H, Hermisson J (2016) The role of recombination in evolutionary rescue. Genetics, 202(2), 721-732.

Uriz MJ, Garate L, Agell G (2017) Molecular phylogenies confirm the presence of two cryptic Hemimycale species in the Mediterranean and reveal the polyphyly of the genera Crella and Hemimycale (Demospongiar: Poecilosclerida). PeerJ, 5, e2958.

Uriz MJ, Maldonado M, Turon X, Marti R (1998) How do reproductive output, larval behaviour, and recruitment contribute to adult spatial patterns in Mediterranean encrusting sponges? Marine Ecology Progress Series, 167, 137-148.

Usher KM, Sutton DC, Toze S, Kuo J, Fromont J (2004) Sexual reproduction in Chondrilla australiensis (Porifera: Demospongiae). Marine and Freshwater Research, 55(2), 123-134.

Uthicke S (2001) Influence of asexual reproduction on the structure and dynamics of Holothuria (Halodeima) atra and Stichopus chloronotus populations of the Great Barrier Reef. Marine and Freshwater Research 52(5), 205-215.

Vacelet J (1999) Planktonic armoured propagules of the excavating sponge Alectona (Porifera: Demospongiae) are larvae: evidence from Alectona wallichii and A. mesatlantica sp. nov. Memoirs of the Queensland Museum, 44, 627-642.

Valentini A, Pompanon F, Taberlet P (2009) DNA barcoding for ecologists. Trends in Ecology and Evolution, 24(2), 110-117.

Van Oosterhout C, Hutchinson WF, Willis DPM, Shipley P (2004) Micro-checker: software for identifying and correcting genotyping errors in microsatellite data. Molecular Ecology Notes, 4, 535-538.

Van Oppen MJH, Worheide G, Takabayashi M (2000) Nuclear markers in evolutionary and population genetic studies of scleractinian corals and sponges. Proceedings of the $9^{\text {th }}$ International Coral Reef Symposium, Bali, Indonesia 23-27 October 2000, Vol. 1.

Van Soest R (2008) Tethya Lamarck, 1815. In: Van Soest RWM, Boury-Esnault N, Hooper JNA, Rützler K, de Voogd NJ, Alvarez B, Hajdu E, Pisera AB, Manconi R, Schönberg C, Klautau M, Picton B, Kelly M, Vacelet J, Dohrmann M, Díaz MC, Cárdenas P, Carballo JL, Ríos P, Downey R (Eds.) World Porifera Database. Accessed at http://www.marinespecies.org/porifera/porifera.php?p=taxdetails\&id=132077 on 2017-10-14. 
Van Soest RWM, Boury-Esnault N, Hooper JNA, Rützler K, de Voogd NJ, Alvarez B, Hajdu E, Pisera AB, Manconi R, Schönberg C, Klautau M, Picton B, Kelly M, Vacelet J, Dohrmann M, Díaz MC, Cárdenas P, Carballo JL, Ríos P, Downey R (2018) World Porifera database. Accessed at http://www.marinespecies.org/porifera on 2018-07-04.

Van Soest RWM, Boury-Esnault N, Vacelet J, Dohrmann M, Erpenbeck D, De Voogd NJ, Santodomingo N, Vanhoorne B, Kelly M, Hooper JNA (2012) Global diversity of sponges (Porifera). PLoS One, 7(4), e35105.

Van Valen L (1976) Ecological species, multispecies, and oaks. Taxon, 25, 233-239.

Van Woesik R (2010) Calm before the spawn: global coral spawning patterns are explained by regional wind fields. Proceedings of the Royal Society B: Biological Sciences, 277(1682), 715722 .

Vance RR (1973) On reproductive strategies in marine benthic invertebrates. The American Naturalist, 107(955), 339-352.

Vange V (2002) Breeding system and inbreeding depression in the clonal plant species Knautia arvensis (Dipsacaceae): implications for survival in abandoned grassland. Biological Conservation, 108(1), 59-67.

Varpe $\varnothing$, Jørgensen C, Tarling GA, Fiksen Ø (2009) The adaptive value of energy storage and capital breeding in seasonal environments. OIKOS, 118(3), 363-370.

Veale AJ (2007) Phylogeography of two intertidal benthic marine invertebrates around New Zealand: the waratah anemone (Actinia tenebrosa) and the snakeskin chiton (Sypharochiton pelliserpentis). MSc Thesis, The University of Auckland, Auckland, New Zealand. URI: https://researchspace.auckland.ac.nz/docs/uoa-docs/rights.htm.

Veale AJ, Lavery SD (2011) Phylogeography of the snakeskin chiton Sypharochiton pelliserpentis (Mollusca: Polypacophora) around New Zealand: are seasonal near-shore upwelling events a dynamic barrier to gene flow? Biological Journal of the Linnean Society, $104,552-563$.

Vergés A, Steinberg PD, Hay ME, Poore AGB, Campbell AH, Ballesteros E, Heck KL, Booth DJ, Coleman MA, Feary DA, Figueria W, Langlois T, Marzinelli EM, Mizerek T, Mumby PJ, Nakamura Y, Roughan M, van Sebille E, Sen Gupta A, Smale DA, Tomas F, Wermberg T, Wilson SK (2014) The tropicalization of temperate marine ecosystems: climate-mediated changes in herbivory and community phase shifts. Proceedings of the Royal Society B, Biological Sciences, 281(1789), 20140846.

Verspoor E, Hammart J (1991) Introgressive hybridization in fishes: the biochemical evidence. Journal of Fish Biology, 39, 309-334.

Visser ME, Both C (2005) Shifts in phenology due to global climate change: the need for a yardstick. Proceedings of the Royal Society B, 272(1581), 2561-2569.

Visser ME, Both C, Lambrechts MM (2004) Global climate change leads to mistimed avian reproduction. Advances in Ecological Research, 35, 89-110. 
Vrijenhoek RC (1989) Genetic and ecological constraints on the origins and establishment of unisexual vertebrates. Evolution and Ecology of Unisexual Vertebrates, 466, 24-31.

Vrijenhoek RC (2009) Cryptic species, phenotypic plasticity, and complex life histories: Assessing deep-sea faunal diversity with molecular markers. Deep-Sea Research Part II: Topical Studies in Oceanography, 56, 1713-1723.

Wallis GP, Cameron-Christie SR, Kennedy HL, Palmer G, Sanders TR, Winter DJ (2017) Interspecific hybridization causes long-term phylogenetic discordance between nuclear and mitochondrial genomes in freshwater fishes. Molecular Ecology, 26, 3116-3127.

Walther GR, Post E, Convey P, Menzel A, Parmesan C, Beebee TJC, Fromentin JM, HoeghGuldberg O, Bairlein F (2002) Ecological responses to recent climate change. Nature 416, 389395.

Waples RS (2015) Testing for Hardy-Weinberg proportions: have we lost the plot? Journal of Heredity 106(1), 1-19.

Warner RR, Cowen RK (2002) Local retention of production in marine populations: evidence, mechanisms and consequences. Bulletin of Marine Science, 70(1), 245-249.

Waugh J (2007). DNA barcoding in animal species: progress, potential and pitfalls. Phylogenetic Systematics, 29, 188-197.

Webster NS, Botte ES, Soo RM, Whalan S (2011) The larval sponge holobiont exhibits high thermal tolerance. Environmental Microbiology Reports, 3(6), 756-762.

Weersing K, Toonen RJ (2009) Population genetics, larval dispersal, and connectivity in marine systems. Marine Ecology Progress Series, 393, 1-12.

Wendel JF, Doyle JJ (1998) Phylogenetic incongruence: Window into genome history and molecular evolution. In: Soltis DE, Soltis PS, Doyle JJ (Eds.) Molecular Systematic of Plants II. Springer: Boston.

Whalan S, Battershill C, de Nys R (2007a) Sexual reproduction of the brooding sponge Rhopaloeides odorabile. Coral Reefs, 26, 655-663.

Whalan S, Battershill C, de Nys R (2007b) Variability in reproductive output across a water quality gradient for a tropical marine sponge, Marine Biology, 153, 163-169.

Whalan S, De Nys R, Smith-Kenune C, Battershill C, Jerry DR (2008) Low genetic variability within and among populations of the brooding sponge Rhodpaloeides odorabile on the central Great Barrier Reef. Aquatic Biology, 3(2), 111-119.

Whalan S, Ettinger-Epstein P, de Nys R (2008) The effect of temperature on larval presettlement duration and metamorphosis for the sponge, Rhopaloeides odorabile. Coral Reefs, 27(4), 738-786.

Wheeler Q, Meier R (Eds.) (2000) Species Concepts and Phylogenetic Theory: A Debate. Columbia University Press. 
Wiese J, Ohlendorf B, Blümel M, Schmaljohann R, Imhoff JF (2011) Phylogenetic identification of fungi isolated from the marine sponge Tethya aurantium and identification of their secondary metabolites. Marine Drugs, 9(4), 561-585.

Wilkinson CR, Evans E (1989) Sponge distribution across Davies Reef, Great Barrier Reef, relative to location, depth and water movement. Coral Reefs, 8(1), 1-7.

Will KW, Rubinoff D (2004) Myth of the molecule: DNA barcodes for species cannot replace morphology for identification and classification. Cladistics, 20(1), 47-55.

Willcox S, Moltschaniwskyj NA, Crawford C (2007) Asexual reproduction in scyphistomae of Aurelia sp.: effects of temperature and salinity in an experimental study. Journal of Experimental Marine Biology and Ecology, 353, 107-114.

Williams SL (2001) Reduced genetic diversity in eelgrass transplantations affects both population growth and individual fitness. Ecological Applications, 11(5), 1472-1488.

Willis PM, Ryan MJ, Rosenthal GG (2011) Encounter rate with conspecific males influence female mate choice in naturally hybridizing fish. Behavioural Ecology, 6(1), 1234-1240.

Wilson K, Hayward BW, Sabaa AT, Scott GH, Kennett JP (2005) A one-million-year history of a north-south segment of the Subtropical Front, east of New Zealand. Paleoceanography, 20, $1-10$.

Witte U (1996) Seasonal reproduction in deep-sea sponges - triggered by vertical particle flux? Marine Biology, 124(4), 571-581.

Witte U, Barthel D, Tendal O (1994) The reproductive cycle of the sponge Halichondria panicea Pallas (1766) and its relationship to temperature and salinity. Journal of Experimental Marine Biology and Ecology, 183, 41-52.

Wörheide G, Epp LS, Macis L (2008) Deep genetic divergence among Indo-Pacific populations of the coral reef sponge Leucetta chagoensis (Leucettidae): founder effects, vicariance, or both? BMC Evolutionary Biology, 8, 24.

Wörheide G, Erpenbeck D (2007) DNA taxonomy of sponges - progress and perspectives. Journal of the Marine Biological Association of the United Kingdom, 87, 1629-1633.

Wulff $\mathbf{J}$ (1986) Variation in clone structure of fragmenting coral reef sponges. Biological Journal of the Linnean Society, 27(4), 311-330.

Wulff J (1991) Asexual fragmentation, genotype success, and population dynamics of erect branching sponges. Journal of Experimental Marine Biology and Ecology, 149(2), 227-247.

Wulff JL (1994) Effects of a hurricane on survival and orientation of large erect coral reef sponges. Coral Reefs, 14(1), 55-61.

Wulff J (2010) Regeneration of sponges in ecological context: is regeneration an integral part of life history and morphological strategies? Integrative and Comparative Biology, 50(4), 494505. 
Xavier JR, Rachello-Dolmen PG, Parra-Velandia F, Schönberg CHL, Breeuwer JAJ, van Soest RWM (2010) Molecular evidence of cryptic speciation in the "cosmopolitan" excavating sponge Cliona celata (Porifera, Clionaidae). Molecular Phylogenetics and Evolution, 56, 1320.

Yamaguchi M (1974) Growth and reproductive cycles of the marine fouling ascidians Ciona intestinalis, Styela plicata, Botrylloides violaceus, and Leptoclinum mitsukurii at AburatsuboMoroiso Inlet (central Japan). Marine Biology, 29(3), 253-259.

Yamahira K (2004) How do multiple environmental cycles in combination determine reproductive timing in marine organisms? A model and test. Functional Ecology, 18, 4-15.

Yin Z, Zhu M, Davidson EH, Bottjer DJ, Zhao F, Tafforeau P (2015) Sponge grade body fossil with cellular resolution dating $60 \mathrm{Myr}$ before the Cambrian. Proceedings of the National Academy of Sciences of the United States of America, 112(12), E1453-E1460.

Yoder JA, McClain CR, Feldman GC, Esaias WE (1993) Annual cycles of phytoplankton chlorophyll concentrations in the global ocean: a satellite view. Global Biogeochemical Cycles, 7(1), 181-193.

Zeger SL, Liang KY (1986) Longitudinal data analysis for discrete and continuous outcomes. Biometrics, 42, 121-130.

Zilberberg C, Sole-Cava AM, Klautau M (2006) The extent of asexual reproduction in sponges of the genus Chondrilla (Demospongiae: Chondrosida) from the Caribbean and the Brazilian coasts. Journal of Experimental Marine Biology and Ecology, 336(2), 211-220.

Zorn CJW (2001) Generalized estimating equation models for correlated data: a review with applications. American Journal of Political Science, 45(2), 470-490. 


\section{APPENDIX 1: \\ INFORMATION FOR SAMPLES USED IN PHYLOGENETIC \\ ANALYSES}

Table A1. Information on collected specimen for Chapter 2. Colour refers to colour of sponge, where $\mathrm{P}=$ pink and $\mathrm{Y}=$ yellow. All sampling sites (Breaker Bay, BB: 41 ${ }^{\circ} 19^{\prime} 53.3^{\prime \prime} \mathrm{S}$ $174^{\circ} 49^{\prime} 52.6^{\prime \prime E}$; Kapiti Island, KP: 4053'23.6"S 174²'40.3"E; Matiu-Somes Island, SI:

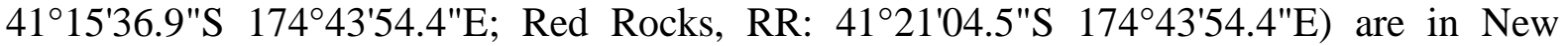
Zealand. All specimens were collected from 5-10 m depth. Phylogenetic clade refers to clades on individual gene phylogenetic tree. GenBank Accession numbers are given in parentheses after haplotype ID.

\begin{tabular}{|c|c|c|c|c|c|c|c|c|}
\hline \multirow{2}{*}{$\begin{array}{l}\text { Sample ID } \\
\text { (field code) }\end{array}$} & \multirow[b]{2}{*}{ Colour } & \multirow{2}{*}{$\begin{array}{l}\text { Sampling } \\
\text { site }\end{array}$} & \multicolumn{3}{|c|}{ Phylogenetic clade } & \multicolumn{3}{|c|}{ Haplotype } \\
\hline & & & $18 \mathrm{~S}$ & $\mathrm{rnl}$ & $\begin{array}{c}\text { COI- } \\
\text { ext }\end{array}$ & $18 \mathrm{~S}$ & $\mathrm{rnl}$ & COI-ext \\
\hline \multicolumn{9}{|c|}{ Tethya bergquistae } \\
\hline BB128 & $\mathrm{P}$ & BB & $\mathrm{C} 1$ & $\mathrm{C} 1$ & $\mathrm{C} 1$ & $\begin{array}{c}\text { 18S-H1 } \\
\text { (MH180010) }\end{array}$ & $\begin{array}{c}\text { rnl-H1 } \\
\text { (MH180013) }\end{array}$ & $\begin{array}{l}\text { COIext-H1 } \\
\text { (MH180020) }\end{array}$ \\
\hline BB124 & $P$ & BB & $\mathrm{C} 1$ & $\mathrm{C} 1$ & $\mathrm{C} 1$ & $\begin{array}{c}\text { 18S-H1 } \\
(\mathrm{MH} 180010)\end{array}$ & $\begin{array}{c}\text { rnl-H1 } \\
(\mathrm{MH} 180013)\end{array}$ & $\begin{array}{c}\text { COIext-H1 } \\
(\mathrm{MH} 180020)\end{array}$ \\
\hline BB142 & $\mathrm{P}$ & BB & $\mathrm{C} 1$ & $\mathrm{C} 1$ & $\mathrm{C} 1$ & $\begin{array}{c}\text { 18S-H1 } \\
(\mathrm{MH} 180010)\end{array}$ & $\begin{array}{c}\mathrm{rnl}-\mathrm{H} 1 \\
(\mathrm{MH} 180013)\end{array}$ & $\begin{array}{c}\text { COIext-H1 } \\
(\mathrm{MH} 180020)\end{array}$ \\
\hline BB151 & $\mathrm{P}$ & BB & $\mathrm{C} 1$ & $\mathrm{C} 1$ & $\mathrm{C} 1$ & $\begin{array}{c}\text { 18S-H1 } \\
(\mathrm{MH} 180010)\end{array}$ & $\begin{array}{c}\mathrm{rnl}-\mathrm{H} 1 \\
(\mathrm{MH} 180013)\end{array}$ & $\begin{array}{c}\text { COIext-H1 } \\
(\mathrm{MH} 180020)\end{array}$ \\
\hline BB117 & $\mathrm{P}$ & BB & $\mathrm{C} 1$ & $\mathrm{C} 1$ & $\mathrm{C} 1$ & $\begin{array}{c}\text { 18S-H1 } \\
(\mathrm{MH} 180010)\end{array}$ & $\begin{array}{c}\text { rnl-H1 } \\
(\mathrm{MH} 180013)\end{array}$ & $\begin{array}{c}\text { COIext-H1 } \\
(\mathrm{MH} 180020)\end{array}$ \\
\hline BB163 & $\mathrm{P}$ & BB & $\mathrm{C} 1$ & $\mathrm{C} 1$ & $\mathrm{C} 1$ & $\begin{array}{c}\text { 18S-H1 } \\
(\mathrm{MH} 180010)\end{array}$ & $\begin{array}{c}\text { rnl-H1 } \\
(\mathrm{MH} 180013)\end{array}$ & $\begin{array}{c}\text { COIext-H1 } \\
(\mathrm{MH} 180020)\end{array}$ \\
\hline BB176 & $\mathrm{P}$ & BB & $\mathrm{C} 1$ & $\mathrm{C} 1$ & $\mathrm{C} 1$ & $\begin{array}{c}\text { 18S-H1 } \\
(\mathrm{MH} 180010)\end{array}$ & $\begin{array}{c}\text { rnl-H1 } \\
(\mathrm{MH} 180013)\end{array}$ & $\begin{array}{l}\text { COIext-H1 } \\
\text { (MH180020) }\end{array}$ \\
\hline BB18 & Y & BB & $\mathrm{C} 1$ & $\mathrm{C} 1$ & $\mathrm{C} 1$ & $\begin{array}{c}\text { 18S-H1 } \\
(\mathrm{MH} 180010)\end{array}$ & $\begin{array}{c}\text { rnl-H1 } \\
(\mathrm{MH} 180013)\end{array}$ & $\begin{array}{l}\text { COIext-H1 } \\
\text { (MH180020) }\end{array}$ \\
\hline BB193 & $\mathrm{P}$ & BB & $\mathrm{C} 1$ & $\mathrm{C} 1$ & $\mathrm{C} 1$ & $\begin{array}{c}\text { 18S-H1 } \\
(\mathrm{MH} 180010)\end{array}$ & $\begin{array}{c}\text { rnl-H1 } \\
(\mathrm{MH} 180013)\end{array}$ & $\begin{array}{l}\text { COIext-H1 } \\
\text { (MH180020) }\end{array}$ \\
\hline BB205 & $\mathrm{P}$ & BB & $\mathrm{C} 1$ & $\mathrm{C} 1$ & $\mathrm{C} 1$ & $\begin{array}{c}\text { 18S-H1 } \\
(\mathrm{MH} 180010)\end{array}$ & $\begin{array}{c}\text { rnl-H1 } \\
(\mathrm{MH} 180013)\end{array}$ & $\begin{array}{c}\text { COIext-H1 } \\
(\mathrm{MH} 180020)\end{array}$ \\
\hline BB16 & $\mathrm{P}$ & BB & $\mathrm{C} 1$ & $\mathrm{C} 1$ & $\mathrm{C} 1$ & $\begin{array}{c}\text { 18S-H1 } \\
(\mathrm{MH} 180010)\end{array}$ & $\begin{array}{c}\text { rnl-H1 } \\
(\mathrm{MH} 180013)\end{array}$ & $\begin{array}{c}\text { COIext-H1 } \\
(\mathrm{MH} 180020)\end{array}$ \\
\hline KP29 & $\mathrm{P}$ & KP & $\mathrm{C} 1$ & $\mathrm{C} 1$ & $\mathrm{C} 1$ & $\begin{array}{c}\text { 18S-H1 } \\
(\mathrm{MH} 180010)\end{array}$ & $\begin{array}{c}\text { rnl-H1 } \\
(\mathrm{MH} 180013)\end{array}$ & $\begin{array}{c}\text { COIext-H1 } \\
(\mathrm{MH} 180020)\end{array}$ \\
\hline KP31 & $\mathrm{P}$ & KP & $\mathrm{C} 1$ & $\mathrm{C} 1$ & $\mathrm{C} 1$ & $\begin{array}{c}\text { 18S-H1 } \\
(\mathrm{MH} 180010)\end{array}$ & $\begin{array}{c}\mathrm{rnl}-\mathrm{H} 1 \\
(\mathrm{MH} 180013)\end{array}$ & $\begin{array}{c}\text { COIext-H1 } \\
(\mathrm{MH} 180020)\end{array}$ \\
\hline KP39 & $P$ & KP & $\mathrm{C} 1$ & $\mathrm{C} 1$ & $\mathrm{C} 1$ & $\begin{array}{c}\text { 18S-H1 } \\
(\mathrm{MH} 180010)\end{array}$ & $\begin{array}{c}\mathrm{rnl}-\mathrm{H} 1 \\
(\mathrm{MH} 180013)\end{array}$ & $\begin{array}{c}\text { COIext-H1 } \\
(\mathrm{MH} 180020)\end{array}$ \\
\hline KP45 & $\mathrm{P}$ & KP & $\mathrm{C} 1$ & $\mathrm{C} 1$ & $\mathrm{C} 1$ & $\begin{array}{c}\text { 18S-H1 } \\
(\mathrm{MH} 180010)\end{array}$ & $\begin{array}{c}\text { rnl-H1 } \\
(\mathrm{MH} 180013)\end{array}$ & $\begin{array}{c}\text { COIext-H1 } \\
(\mathrm{MH} 180020)\end{array}$ \\
\hline BB9 & $\mathrm{Y}$ & BB & $\mathrm{C} 1$ & $\mathrm{C} 1$ & $\mathrm{C} 1$ & $\begin{array}{c}\text { 18S-H1 } \\
(\mathrm{MH} 180010)\end{array}$ & $\begin{array}{c}\text { rnl-H1 } \\
(\mathrm{MH} 180013)\end{array}$ & $\begin{array}{c}\text { COIext-H1 } \\
(\mathrm{MH} 180020)\end{array}$ \\
\hline KP46 & $\mathrm{P}$ & KP & $\mathrm{C} 1$ & $\mathrm{C} 1$ & $\mathrm{C} 1$ & $\begin{array}{c}\text { 18S-H1 } \\
(\mathrm{MH} 180010)\end{array}$ & $\begin{array}{c}\mathrm{rnl}-\mathrm{H} 1 \\
(\mathrm{MH} 180013)\end{array}$ & $\begin{array}{c}\text { COIext-H1 } \\
\text { (MH180020) }\end{array}$ \\
\hline \multicolumn{9}{|c|}{ Tethya burtoni } \\
\hline BB255 & Y & BB & $\mathrm{C} 2$ & $\mathrm{C} 2$ & $\mathrm{C} 2$ & $\begin{array}{c}\text { 18S-H2 } \\
(\mathrm{MH} 180011)\end{array}$ & $\begin{array}{c}\text { rnl-H3 } \\
\text { (MH180015) }\end{array}$ & $\begin{array}{l}\text { COIext-H2 } \\
\text { (MH180021) }\end{array}$ \\
\hline
\end{tabular}




\begin{tabular}{|c|c|c|c|c|c|c|c|c|}
\hline BB258 & Y & BB & $\mathrm{C} 2$ & $\mathrm{C} 2$ & $\mathrm{C} 2$ & $\begin{array}{c}\text { 18S-H2 } \\
\text { (MH180011) }\end{array}$ & $\begin{array}{c}\text { rnl-H3 } \\
(\mathrm{MH} 180015)\end{array}$ & $\begin{array}{c}\text { COIext-H2 } \\
\text { (MH180021) }\end{array}$ \\
\hline BB270 & Y & BB & $\mathrm{C} 2$ & $\mathrm{C} 2$ & $\mathrm{C} 2$ & $\begin{array}{c}\text { 18S-H2 } \\
(\mathrm{MH} 180011)\end{array}$ & $\begin{array}{c}\text { rnl-H3 } \\
(\mathrm{MH} 180015)\end{array}$ & $\begin{array}{c}\text { COIext-H2 } \\
(\mathrm{MH} 180021)\end{array}$ \\
\hline BB264 & Y & BB & $\mathrm{C} 2$ & $\mathrm{C} 2$ & $\mathrm{C} 2$ & $\begin{array}{c}\text { 18S-H2 } \\
(\mathrm{MH} 180011)\end{array}$ & $\begin{array}{c}\text { rnl-H4 } \\
(\mathrm{MH} 180016)\end{array}$ & $\begin{array}{c}\text { COIext-H2 } \\
(\mathrm{MH} 180021)\end{array}$ \\
\hline BB265 & Y & BB & $\mathrm{C} 2$ & $\mathrm{C} 2$ & $\mathrm{C} 2$ & $\begin{array}{c}\text { 18S-H2 } \\
(\mathrm{MH} 180011)\end{array}$ & $\begin{array}{c}\text { rnl-H3 } \\
(\mathrm{MH} 180015)\end{array}$ & $\begin{array}{c}\text { COIext-H2 } \\
(\mathrm{MH} 180021)\end{array}$ \\
\hline KP63 & Y & KP & $\mathrm{C} 2$ & $\mathrm{C} 2$ & $\mathrm{C} 2$ & $\begin{array}{c}\text { 18S-H2 } \\
(\mathrm{MH} 180011)\end{array}$ & $\begin{array}{c}\text { rnl-H3 } \\
(\mathrm{MH} 180015)\end{array}$ & $\begin{array}{c}\text { COIext-H2 } \\
(\mathrm{MH} 180021)\end{array}$ \\
\hline KP66 & Y & KP & $\mathrm{C} 2$ & $\mathrm{C} 2$ & $\mathrm{C} 2$ & $\begin{array}{c}\text { 18S-H2 } \\
(\mathrm{MH} 180011)\end{array}$ & $\begin{array}{c}\text { rnl-H4 } \\
(\mathrm{MH} 180016)\end{array}$ & $\begin{array}{c}\text { COIext-H2 } \\
(\mathrm{MH} 180021)\end{array}$ \\
\hline SI324 & Y & SI & $\mathrm{C} 2$ & $\mathrm{C} 2$ & C3 & $\begin{array}{c}\text { 18S-H2 } \\
(\mathrm{MH} 180011)\end{array}$ & $\begin{array}{c}\text { rnl-H4 } \\
(\mathrm{MH} 180016)\end{array}$ & $\begin{array}{c}\text { COIext-H3 } \\
(\mathrm{MH} 180023)\end{array}$ \\
\hline KP80 & Y & KP & $\mathrm{C} 2$ & $\mathrm{C} 2$ & C3 & $\begin{array}{c}\text { 18S-H2 } \\
(\mathrm{MH} 180011)\end{array}$ & $\begin{array}{c}\text { rnl-H4 } \\
(\mathrm{MH} 180016)\end{array}$ & $\begin{array}{c}\text { COIext-H4 } \\
\text { (MH180022) }\end{array}$ \\
\hline KP78 & Y & KP & $\mathrm{C} 2$ & $\mathrm{C} 2$ & $\mathrm{C} 3$ & $\begin{array}{c}\text { 18S-H2 } \\
(\mathrm{MH} 180011)\end{array}$ & $\begin{array}{c}\text { rnl-H3 } \\
(\mathrm{MH} 180015)\end{array}$ & $\begin{array}{c}\text { COIext-H4 } \\
(\mathrm{MH} 180022)\end{array}$ \\
\hline KP68 & Y & KP & $\mathrm{C} 2$ & $\mathrm{C} 2$ & $\mathrm{C} 2$ & $\begin{array}{c}\text { 18S-H2 } \\
(\mathrm{MH} 180011)\end{array}$ & $\begin{array}{c}\mathrm{rnl}-\mathrm{H} 3 \\
(\mathrm{MH} 180015)\end{array}$ & $\begin{array}{c}\text { COIext-H2 } \\
(\mathrm{MH} 180021)\end{array}$ \\
\hline KP69 & Y & KP & $\mathrm{C} 2$ & $\mathrm{C} 2$ & $\mathrm{C} 2$ & $\begin{array}{c}\text { 18S-H2 } \\
(\mathrm{MH} 180011)\end{array}$ & $\begin{array}{c}\text { rnl-H3 } \\
(\mathrm{MH} 180015)\end{array}$ & $\begin{array}{c}\text { COIext-H2 } \\
(\mathrm{MH} 180021)\end{array}$ \\
\hline KP70 & Y & KP & $\mathrm{C} 2$ & $\mathrm{C} 2$ & $\mathrm{C} 2$ & $\begin{array}{c}\text { 18S-H2 } \\
(\mathrm{MH} 180011)\end{array}$ & $\begin{array}{c}\text { rnl-H3 } \\
(\mathrm{MH} 180015)\end{array}$ & $\begin{array}{c}\text { COIext-H2 } \\
(\mathrm{MH} 180021)\end{array}$ \\
\hline SI321 & Y & SI & $\mathrm{C} 2$ & $\mathrm{C} 2$ & $\mathrm{C} 2$ & $\begin{array}{c}\text { 18S-H2 } \\
(\mathrm{MH} 180011)\end{array}$ & $\begin{array}{c}\text { rnl-H4 } \\
(\mathrm{MH} 180016)\end{array}$ & $\begin{array}{c}\text { COIext-H2 } \\
(\mathrm{MH} 180021)\end{array}$ \\
\hline SI317 & Y & SI & $\mathrm{C} 2$ & $\mathrm{C} 2$ & $\mathrm{C} 2$ & $\begin{array}{c}\text { 18S-H2 } \\
(\mathrm{MH} 180011)\end{array}$ & $\begin{array}{c}\text { rnl-H3 } \\
(\mathrm{MH} 180015)\end{array}$ & $\begin{array}{c}\text { COIext-H2 } \\
(\mathrm{MH} 180021)\end{array}$ \\
\hline KP75 & Y & KP & $\mathrm{C} 2$ & $\mathrm{C} 2$ & $\mathrm{C} 2$ & $\begin{array}{c}\text { 18S-H2 } \\
(\mathrm{MH} 180011)\end{array}$ & $\begin{array}{c}\text { rnl-H3 } \\
(\mathrm{MH} 180015)\end{array}$ & $\begin{array}{c}\text { COIext-H2 } \\
(\mathrm{MH} 180021)\end{array}$ \\
\hline SI314 & Y & SI & $\mathrm{C} 2$ & $\mathrm{C} 2$ & C2 & $\begin{array}{c}\text { 18S-H2 } \\
(\mathrm{MH} 180011)\end{array}$ & $\begin{array}{c}\text { rnl-H4 } \\
(\mathrm{MH} 180016)\end{array}$ & $\begin{array}{c}\text { COIext-H2 } \\
(\mathrm{MH} 180021)\end{array}$ \\
\hline KP76 & Y & KP & $\mathrm{C} 2$ & $\mathrm{C} 2$ & C3 & $\begin{array}{c}\text { 18S-H2 } \\
(\mathrm{MH} 180011)\end{array}$ & $\begin{array}{c}\text { rnl-H3 } \\
(\mathrm{MH} 180015)\end{array}$ & $\begin{array}{c}\text { COIext-H4 } \\
\text { (MH180022) }\end{array}$ \\
\hline SI313 & Y & SI & $\mathrm{C} 2$ & $\mathrm{C} 2$ & $\mathrm{C} 3$ & $\begin{array}{c}\text { 18S-H2 } \\
(\mathrm{MH} 180011)\end{array}$ & $\begin{array}{c}\text { rnl-H4 } \\
(\mathrm{MH} 180016)\end{array}$ & $\begin{array}{c}\text { COIext-H4 } \\
\text { (MH180022) }\end{array}$ \\
\hline SI309 & Y & SI & $\mathrm{C} 2$ & $\mathrm{C} 2$ & C3 & $\begin{array}{c}\text { 18S-H2 } \\
(\mathrm{MH} 180011)\end{array}$ & $\begin{array}{c}\text { rnl-H4 } \\
(\mathrm{MH} 180016)\end{array}$ & $\begin{array}{c}\text { COIext-H4 } \\
\text { (MH180022) }\end{array}$ \\
\hline SI303 & Y & SI & $\mathrm{C} 2$ & $\mathrm{C} 2$ & C3 & $\begin{array}{c}\text { 18S-H2 } \\
(\mathrm{MH} 180011)\end{array}$ & $\begin{array}{c}\text { rnl-H4 } \\
(\mathrm{MH} 180016)\end{array}$ & $\begin{array}{c}\text { COIext-H4 } \\
\text { (MH180022) }\end{array}$ \\
\hline SI302 & Y & SI & $\mathrm{C} 2$ & $\mathrm{C} 2$ & C3 & $\begin{array}{c}\text { 18S-H2 } \\
(\mathrm{MH} 180011)\end{array}$ & $\begin{array}{c}\text { rnl-H3 } \\
(\mathrm{MH} 180015)\end{array}$ & $\begin{array}{c}\text { COIext-H4 } \\
\text { (MH180022) }\end{array}$ \\
\hline RR377 & $\mathrm{Y}$ & $\mathrm{RR}$ & $\mathrm{C} 2$ & $\mathrm{C} 2$ & $\mathrm{C} 3$ & $\begin{array}{c}\text { 18S-H2 } \\
(\mathrm{MH} 180011)\end{array}$ & $\begin{array}{c}\text { rnl-H4 } \\
(\mathrm{MH} 180016)\end{array}$ & $\begin{array}{c}\text { COIext-H3 } \\
\text { (MH180023) }\end{array}$ \\
\hline KP61 & Y & KP & $\mathrm{C} 2$ & $\mathrm{C} 2$ & $\mathrm{C} 2$ & $\begin{array}{c}\text { 18S-H2 } \\
(\mathrm{MH} 180011)\end{array}$ & $\begin{array}{c}\text { rnl-H3 } \\
(\mathrm{MH} 180015)\end{array}$ & $\begin{array}{c}\text { COIext-H2 } \\
\text { (MH180021) }\end{array}$ \\
\hline KP60 & Y & KP & $\mathrm{C} 2$ & $\mathrm{C} 2$ & $\mathrm{C} 2$ & $\begin{array}{c}\text { 18S-H2 } \\
(\mathrm{MH} 180011)\end{array}$ & $\begin{array}{c}\text { rnl-H2 } \\
(\mathrm{MH} 180014)\end{array}$ & $\begin{array}{c}\text { COIext-H2 } \\
\text { (MH180021) }\end{array}$ \\
\hline KP58 & Y & KP & $\mathrm{C} 2$ & $\mathrm{C} 2$ & C2 & $\begin{array}{c}\text { 18S-H2 } \\
(\mathrm{MH} 180011)\end{array}$ & $\begin{array}{c}\text { rnl-H3 } \\
(\mathrm{MH} 180015)\end{array}$ & $\begin{array}{c}\text { COIext-H2 } \\
\text { (MH180021) }\end{array}$ \\
\hline KP57 & $\mathrm{Y}$ & KP & $\mathrm{C} 2$ & $\mathrm{C} 2$ & $\mathrm{C} 2$ & $\begin{array}{c}\text { 18S-H2 } \\
(\mathrm{MH} 180011)\end{array}$ & $\begin{array}{c}\text { rnl-H4 } \\
(\mathrm{MH} 180016)\end{array}$ & $\begin{array}{c}\text { COIext-H2 } \\
(\mathrm{MH} 180021)\end{array}$ \\
\hline KP52 & Y & KP & $\mathrm{C} 2$ & $\mathrm{C} 2$ & $\mathrm{C} 2$ & $\begin{array}{c}\text { 18S-H2 } \\
(\mathrm{MH} 180011)\end{array}$ & $\begin{array}{c}\text { rnl-H3 } \\
(\mathrm{MH} 180015)\end{array}$ & $\begin{array}{c}\text { COIext-H2 } \\
(\mathrm{MH} 180021)\end{array}$ \\
\hline KP51 & Y & KP & $\mathrm{C} 2$ & $\mathrm{C} 2$ & $\mathrm{C} 2$ & $\begin{array}{c}\text { 18S-H2 } \\
(\mathrm{MH} 180011)\end{array}$ & $\begin{array}{c}\mathrm{rnl}-\mathrm{H} 3 \\
(\mathrm{MH} 180015)\end{array}$ & $\begin{array}{c}\text { COIext-H2 } \\
(\mathrm{MH} 180021)\end{array}$ \\
\hline RR375 & Y & RR & $\mathrm{C} 2$ & $\mathrm{C} 2$ & C3 & $\begin{array}{c}\text { 18S-H2 } \\
(\mathrm{MH} 180011)\end{array}$ & $\begin{array}{c}\text { rnl-H3 } \\
(\mathrm{MH} 180015)\end{array}$ & $\begin{array}{c}\text { COIext-H3 } \\
(\mathrm{MH} 180023)\end{array}$ \\
\hline SI300 & Y & SI & $\mathrm{C} 2$ & $\mathrm{C} 2$ & C3 & $\begin{array}{c}\text { 18S-H2 } \\
(\mathrm{MH} 180011)\end{array}$ & $\begin{array}{c}\text { rnl-H4 } \\
(\mathrm{MH} 180016)\end{array}$ & $\begin{array}{c}\text { COIext-H4 } \\
(\mathrm{MH} 180022)\end{array}$ \\
\hline
\end{tabular}




\begin{tabular}{|c|c|c|c|c|c|c|c|c|}
\hline SI295 & Y & SI & $\mathrm{C} 2$ & $\mathrm{C} 2$ & $\mathrm{C} 2$ & $\begin{array}{c}\text { 18S-H2 } \\
(\mathrm{MH} 180011)\end{array}$ & $\begin{array}{c}\text { rnl-H3 } \\
\text { (MH180015) }\end{array}$ & $\begin{array}{l}\text { COIext-H2 } \\
\text { (MH180021) }\end{array}$ \\
\hline RR385 & Y & RR & $\mathrm{C} 2$ & $\mathrm{C} 2$ & C3 & $\begin{array}{c}\text { 18S-H2 } \\
(\mathrm{MH} 180011)\end{array}$ & $\begin{array}{c}\text { rnl-H4 } \\
(\mathrm{MH} 180016)\end{array}$ & $\begin{array}{c}\text { COIext-H3 } \\
(\mathrm{MH} 180023)\end{array}$ \\
\hline SI310 & Y & SI & $\mathrm{C} 2$ & $\mathrm{C} 2$ & C3 & $\begin{array}{c}\text { 18S-H2 } \\
(\mathrm{MH} 180011)\end{array}$ & $\begin{array}{c}\text { rnl-H4 } \\
(\mathrm{MH} 180016)\end{array}$ & $\begin{array}{c}\text { COIext-H4 } \\
(\mathrm{MH} 180022)\end{array}$ \\
\hline KP77 & Y & KP & $\mathrm{C} 2$ & $\mathrm{C} 2$ & $\mathrm{C} 3$ & $\begin{array}{c}\text { 18S-H2 } \\
(\mathrm{MH} 180011)\end{array}$ & $\begin{array}{c}\text { rnl-H3 } \\
(\mathrm{MH} 180015)\end{array}$ & $\begin{array}{c}\text { COIext-H4 } \\
\text { (MH180022) }\end{array}$ \\
\hline KP71 & Y & KP & $\mathrm{C} 2$ & $\mathrm{C} 2$ & $\mathrm{C} 2$ & $\begin{array}{c}\text { 18S-H2 } \\
(\mathrm{MH} 180011)\end{array}$ & $\begin{array}{c}\text { rnl-H3 } \\
(\mathrm{MH} 180015)\end{array}$ & $\begin{array}{c}\text { COIext-H2 } \\
(\mathrm{MH} 180021)\end{array}$ \\
\hline RR372 & Y & $\mathrm{RR}$ & C3 & C3 & C3 & $\begin{array}{c}\text { 18S-H3 } \\
(\mathrm{MH} 180012)\end{array}$ & $\begin{array}{c}\text { rnl-H7 } \\
(\text { MH180019) }\end{array}$ & $\begin{array}{c}\text { COIext-H3 } \\
(\mathrm{MH} 180023)\end{array}$ \\
\hline RR384 & Y & RR & C3 & C3 & C3 & $\begin{array}{c}\text { 18S-H3 } \\
(\mathrm{MH} 180012)\end{array}$ & $\begin{array}{c}\text { rnl-H7 } \\
(\text { MH180019) }\end{array}$ & $\begin{array}{c}\text { COIext-H3 } \\
(\mathrm{MH} 180023)\end{array}$ \\
\hline KP62 & Y & KP & $\mathrm{C} 3$ & C3 & $\mathrm{C} 3$ & $\begin{array}{c}\text { 18S-H3 } \\
(\mathrm{MH} 180012)\end{array}$ & $\begin{array}{c}\text { rnl-H6 } \\
(\text { MH180018) }\end{array}$ & $\begin{array}{c}\text { COIext-H3 } \\
(\mathrm{MH} 180023)\end{array}$ \\
\hline KP43 & Y & KP & C3 & $\mathrm{C} 3$ & C3 & $\begin{array}{c}\text { 18S-H3 } \\
(\mathrm{MH} 180012)\end{array}$ & $\begin{array}{c}\text { rnl-H5 } \\
(\mathrm{MH} 180017)\end{array}$ & $\begin{array}{c}\text { COIext-H3 } \\
(\mathrm{MH} 180023)\end{array}$ \\
\hline BB280 & Y & BB & C3 & $\mathrm{C} 3$ & C3 & $\begin{array}{c}\text { 18S-H3 } \\
(\mathrm{MH} 180012)\end{array}$ & $\begin{array}{c}\text { rnl-H7 } \\
(\mathrm{MH} 180019)\end{array}$ & $\begin{array}{l}\text { COIext-H4 } \\
\text { (MH180022) }\end{array}$ \\
\hline BB275 & Y & BB & C3 & C3 & C3 & $\begin{array}{c}\text { 18S-H3 } \\
(\mathrm{MH} 180012)\end{array}$ & $\begin{array}{c}\text { rnl-H7 } \\
(\mathrm{MH} 180019)\end{array}$ & $\begin{array}{c}\text { COIext-H4 } \\
\text { (MH180022) }\end{array}$ \\
\hline BB167 & Y & BB & C3 & C3 & C3 & $\begin{array}{c}\text { 18S-H3 } \\
(\mathrm{MH} 180012)\end{array}$ & $\begin{array}{c}\text { rnl-H5 } \\
(\mathrm{MH} 180017)\end{array}$ & $\begin{array}{c}\text { COIext-H3 } \\
(\mathrm{MH} 180023)\end{array}$ \\
\hline BB252 & Y & BB & C3 & C3 & C3 & $\begin{array}{c}\text { 18S-H3 } \\
(\mathrm{MH} 180012)\end{array}$ & $\begin{array}{c}\text { rnl-H5 } \\
(\mathrm{MH} 180017)\end{array}$ & $\begin{array}{c}\text { COIext-H3 } \\
(\mathrm{MH} 180023)\end{array}$ \\
\hline BB254 & Y & BB & $\mathrm{C} 3$ & $\mathrm{C} 3$ & $\mathrm{C} 3$ & $\begin{array}{c}\text { 18S-H3 } \\
(\mathrm{MH} 180012)\end{array}$ & $\begin{array}{c}\text { rnl-H7 } \\
(\text { MH180019) }\end{array}$ & $\begin{array}{c}\text { COIext-H4 } \\
(\mathrm{MH} 180022)\end{array}$ \\
\hline BB256 & Y & BB & C3 & C3 & C3 & $\begin{array}{c}\text { 18S-H3 } \\
(\mathrm{MH} 180012)\end{array}$ & $\begin{array}{c}\text { rnl-H7 } \\
(\text { MH180019) }\end{array}$ & $\begin{array}{c}\text { COIext-H4 } \\
(\mathrm{MH} 180022)\end{array}$ \\
\hline BB257 & Y & BB & C3 & C3 & C3 & $\begin{array}{c}\text { 18S-H3 } \\
(\mathrm{MH} 180012)\end{array}$ & $\begin{array}{c}\text { rnl-H7 } \\
(\mathrm{MH} 180019)\end{array}$ & $\begin{array}{c}\text { COIext-H4 } \\
(\mathrm{MH} 180022)\end{array}$ \\
\hline BB260 & Y & BB & C3 & C3 & C3 & $\begin{array}{c}\text { 18S-H3 } \\
(\mathrm{MH} 180012)\end{array}$ & $\begin{array}{c}\text { rnl-H7 } \\
(\mathrm{MH} 180019)\end{array}$ & $\begin{array}{c}\text { COIext-H4 } \\
(\mathrm{MH} 180022)\end{array}$ \\
\hline BB272 & Y & BB & C3 & $\mathrm{C} 3$ & C3 & $\begin{array}{c}\text { 18S-H3 } \\
(\mathrm{MH} 180012)\end{array}$ & $\begin{array}{c}\text { rnl-H7 } \\
(\mathrm{MH} 180019)\end{array}$ & $\begin{array}{c}\text { COIext-H4 } \\
\text { (MH180022) }\end{array}$ \\
\hline BB271 & Y & BB & C3 & C3 & C3 & $\begin{array}{c}18 \mathrm{~S}-\mathrm{H} 3 \\
(\mathrm{MH} 180012)\end{array}$ & $\begin{array}{c}\text { rnl-H6 } \\
(\mathrm{MH} 180018)\end{array}$ & $\begin{array}{l}\text { COIext-H3 } \\
\text { (MH180023) }\end{array}$ \\
\hline BB263 & Y & BB & C3 & C3 & C3 & $\begin{array}{c}\text { 18S-H3 } \\
\text { (MH180012) }\end{array}$ & $\begin{array}{c}\text { rnl-H7 } \\
(\mathrm{MH} 180019)\end{array}$ & $\begin{array}{c}\text { COIext-H4 } \\
(\mathrm{MH} 180022)\end{array}$ \\
\hline \multicolumn{9}{|c|}{$\begin{array}{c}\text { Tethya bergquistae-burtoni } \\
\text { hybrids }\end{array}$} \\
\hline KP72 & Y & KP & $\mathrm{C} 1$ & $\mathrm{C} 1$ & $\mathrm{C} 2$ & $\begin{array}{c}\text { 18S-H1 } \\
\text { (MH180010) }\end{array}$ & $\begin{array}{c}\text { rnl-H1 } \\
(\mathrm{MH} 180013)\end{array}$ & $\begin{array}{l}\text { COIext-H2 } \\
\text { (MH180021) }\end{array}$ \\
\hline BB177 & Y & BB & $\mathrm{C} 2$ & $\mathrm{C} 2$ & $\mathrm{C} 1$ & $\begin{array}{c}18 \mathrm{~S}-\mathrm{H} 2 \\
(\mathrm{MH} 180011)\end{array}$ & $\begin{array}{c}\text { rnl-H3 } \\
(\mathrm{MH} 180015)\end{array}$ & $\begin{array}{l}\text { COIext-H1 } \\
\text { (MH180020) }\end{array}$ \\
\hline SI322 & Y & SI & $\mathrm{C} 2$ & $\mathrm{C} 2$ & $\mathrm{C} 1$ & $\begin{array}{c}\text { 18S-H2 } \\
(\mathrm{MH} 180011)\end{array}$ & $\begin{array}{c}\text { rnl-H4 } \\
(\mathrm{MH} 180016)\end{array}$ & $\begin{array}{c}\text { COIext-H1 } \\
\text { (MH180020) }\end{array}$ \\
\hline
\end{tabular}




\section{APPENDIX 2: \\ DEFINITION OF SPICULE TYPES}

Spicule type was assigned using descriptions provided in Bergquist and Kelly-Borges (1991) and Sarà and Sarà (2004). The following are definitions for the observed spicule types given in these studies:

\section{$\underline{\text { Megascleres }}$}

Megascleres contained in Tethya spp. were strongyloxea and anisostrongyles (also known as anisostrongyloxeas). Strongyloxea is used to describe a style which has two different ends (e.g., the distal end is different to the proximal end). Anisostrongyles are a certain type of strongyloxea in which the distal end is rounded and smaller than the proximal end. We also observed a smaller, thinner auxiliary style, which we refer to as simply 'style.'

\section{$\underline{\text { Microscleres }}$}

Microscleres observed were megasters $(\sim 40-50 \mu \mathrm{m})$ and micrasters $(\sim 5-15 \mu \mathrm{m})$. Megasters included spherasters and oxyspherasters. Spherasters are distinguishable from oxyspherasters in that the former have an R:C ratio (e.g., ratio between ray length and diameter of the centre) less than or equal to 1 , whereas the latter has an $\mathrm{R}: \mathrm{C}$ ratio greater than 1 but less than 3 . Micrasters included oxyasters (also known as oxyspheraters), tylasters, chiasters and strongylasters. Oxyasters are star-shaped asters, with conical rays, whereas tylasters are asters with knobbed ends that are generally spined. Both chiasters and strongylasters are asters that are an intermediate between oxyasters and tylasters, with the distinction that strongylasters contain rounded ray tips. These can also be referred to as acanthooxyasters, acanthochiasters, acanthotylasters, or acanthostrongylasters, where the prefix "acantho-" means containing spines. 
APPENDIX 3:

CORRELATION OF ENVIRONMENTAL COVARIATES

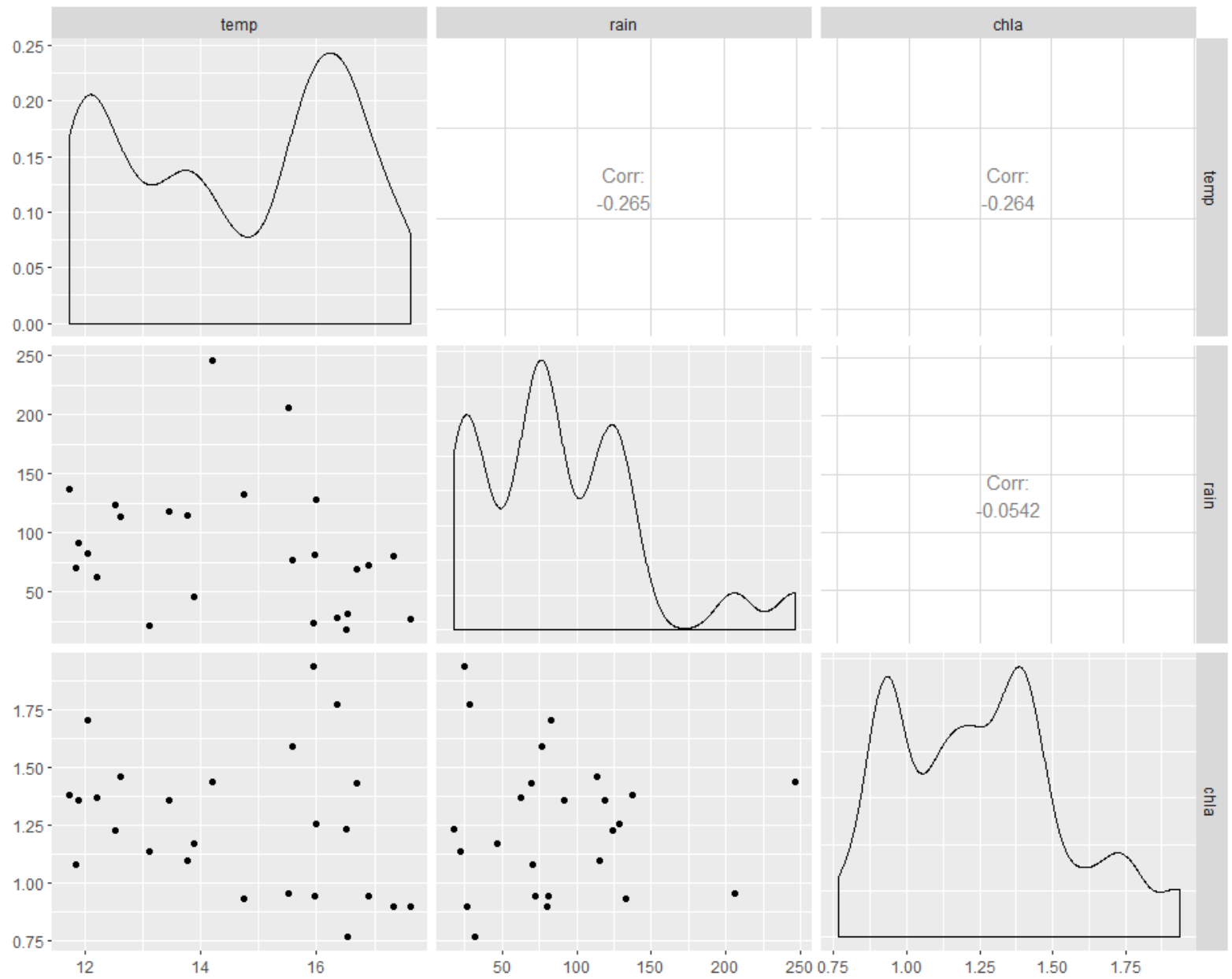

Figure A3. Pairwise correlations between monthly means of sea surface temperature (temp), total monthly rainfall (rain), and monthly means of chlorophyll- $a$ concentration (chla). 


\section{APPENDIX 4:}

\section{DIFFERENCES IN ROI BETWEEN SITE AND SPECIES}
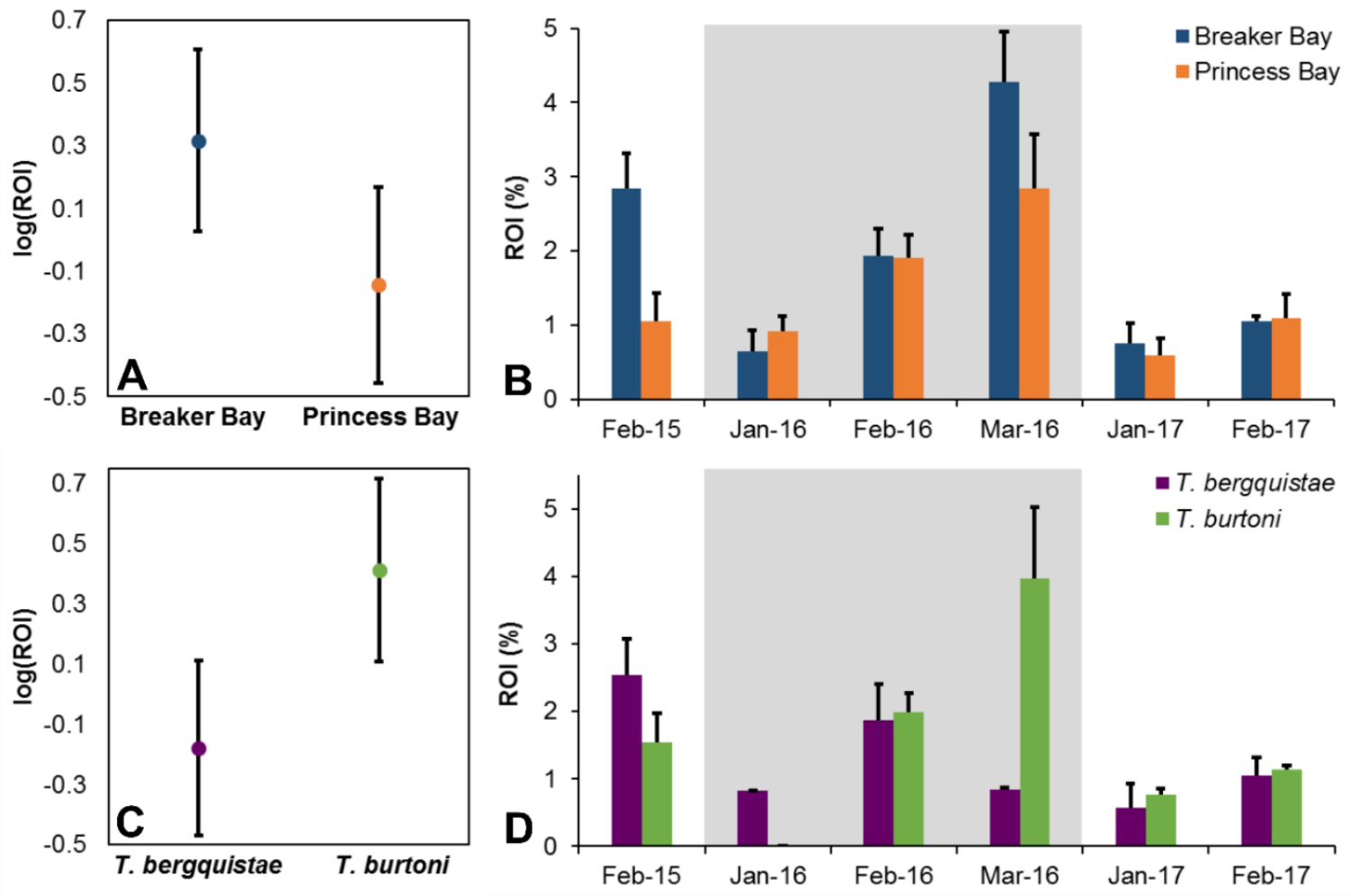

Figure A4. The effects of site (top) and species (bottom) on ROI for Tethya bergquistae (purple) and T. burtoni (green) from both Breaker Bay (blue) and Princess Bay (orange). ROI, or the reproductive output index, corresponds to the percent of tissue containing oocytes. Plots on left are means of the $\log (\mathrm{ROI})$ between site (A) and species (C), and bars are $95 \%$ confidence intervals. Plots on right are differences in ROI between sites (B) and species (D) presented by month, where the entire reproductive event captured is highlighted in grey. Sample sizes for species collected from each site are presented in Table 3.1. Error bars on plots on right correspond to standard error. 


\section{APPENDIX 5:}

\section{SUMMARIES OF RESULTS FROM ANOVAs AND LR $\chi^{2}$ TESTS FROM CHAPTERS 3 AND 4}

Table A5a. Results of likelihood ratio chi-square tests, testing the effects of site, sponge size, species, sea surface temperature, rainfall and chlorophyll- $a$ concentration on the probability of a sponge containing gametes (Chapter 3 ). On the left, temperature as a continuous variable; on the right, temperature as a categorical variable.

\begin{tabular}{|lccc|lccc|}
\hline \multicolumn{5}{|l}{ Probability of sexual reproduction } & \multicolumn{5}{l|}{} & & & \\
\hline & $d f$ & $\chi^{2}$ & $P$ & & $d f$ & $\chi^{2}$ & $P$ \\
\hline Site & 1 & 0.089 & 0.765 & Site & 1 & 0.177 & 0.674 \\
\hline Size & 1 & 12.416 & $\mathbf{< 0 . 0 0 1}$ & Size & 1 & 12.940 & $<\mathbf{0 . 0 0 1}$ \\
\hline Species & 1 & 0.058 & 0.810 & Species & 1 & 0.033 & 0.856 \\
\hline SST (contin.) & 1 & 93.221 & $\mathbf{< 0 . 0 0 1}$ & SST (cat.) & 1 & 78.268 & $<\mathbf{0 . 0 0 1}$ \\
\hline Rainfall & 1 & 7.568 & $\mathbf{0 . 0 0 6}$ & Rainfall & 1 & 14.893 & $<\mathbf{0 . 0 0 1}$ \\
\hline Chl- $a$ & 1 & 0.401 & 0.527 & Chl- $a$ & 1 & 0.856 & 0.355 \\
\hline
\end{tabular}

Table A5b. Results of an ANOVA testing effects of site, sponge size and species on oocyte density, ROI (or a reproductive output index, or the \% of tissue occupied by gametes) and oocyte size (Chapter 3 ).

\begin{tabular}{|l|ccc|ccc|ccc|}
\hline & \multicolumn{3}{|c|}{ Oocyte density } & \multicolumn{4}{c|}{ ROI } & \multicolumn{3}{c|}{ Oocyte size } \\
& $d f$ & $\mathrm{~F}$ & $P$ & $d f$ & $\mathrm{~F}$ & $P$ & $d f$ & $\mathrm{~F}$ & $P$ \\
\hline Site & 1 & 10.593 & $\mathbf{0 . 0 0 2}$ & 1 & 8.7105 & $\mathbf{0 . 0 0 4}$ & 1 & 0.6179 & 0.434 \\
Size & 1 & 7.177 & $\mathbf{0 . 0 0 9}$ & 1 & 6.3607 & $\mathbf{0 . 0 1 4}$ & 1 & 1.3163 & 0.255 \\
Species & 1 & 13.073 & $\mathbf{0 . 0 0 1}$ & 1 & 7.7786 & $\mathbf{0 . 0 0 7}$ & 1 & 1.1046 & 0.296 \\
Residuals & 82 & & & 82 & & & 82 & & \\
\hline
\end{tabular}


Table A5c. Results of likelihood ratio chi-square tests, testing the main effects of site, sponge size, species, sea surface temperature, rainfall and chlorophyll- $a$ concentrations on the probability of a sponge containing buds (Chapter 4). On the left, temperature as a continuous variable; on the right, temperature as a categorical variable.

\begin{tabular}{|c|c|c|c|c|c|c|c|}
\hline \multicolumn{8}{|c|}{ Probability of budding } \\
\hline & $d f$ & $\chi^{2}$ & $P$ & & $d f$ & $\chi^{2}$ & $P$ \\
\hline Site & 1 & 6.264 & 0.012 & Site & 1 & 6.133 & 0.013 \\
\hline Size & 1 & 11.115 & $<0.001$ & Size & 1 & 11.313 & $<0.001$ \\
\hline Species & 1 & 30.053 & $<0.001$ & Species & 1 & 29.536 & $<0.001$ \\
\hline SST (contin.) & 1 & 93.369 & $<0.001$ & SST (cat.) & 1 & 80.070 & $<0.001$ \\
\hline Rainfall & 1 & 0.138 & 0.710 & Rainfall & 1 & 0.619 & 0.431 \\
\hline Chl- $a$ & 1 & 1.380 & 0.240 & Chl- $a$ & 1 & 0.055 & 0.815 \\
\hline
\end{tabular}

Table A5d. Results of likelihood ratio chi-square for effects from the best fit model for the probability of budding. Species refers to either Tethya bergquistae or T. burtoni, site refers to either Breaker Bay or Princess Bay (Chapter 4). Sea surface temperature (SST) in ${ }^{\circ} \mathrm{C}$ and chlorophyll- $a$ concentrations in $\mathrm{mg} / \mathrm{m}^{3}$.

\begin{tabular}{|lccc|}
\hline \multicolumn{4}{|l}{ Probability of budding } \\
& $d f$ & $\chi^{2}$ & $P$ \\
\hline Site $\times$ Species & 1 & 10.609 & $\mathbf{0 . 0 0 1}$ \\
\hline Species $\times$ Size & 1 & 16.714 & $<\mathbf{0 . 0 0 1}$ \\
\hline SST $\times$ Chl- $a$ & 1 & 5.226 & $\mathbf{0 . 0 2 2}$ \\
\hline Chl $-a \times$ Species & 1 & 6.525 & $\mathbf{0 . 0 1 1}$ \\
\hline
\end{tabular}


Table A5e. Results from a Type-III ANOVA, testing the main effects of site, sponge size, species, sea surface temperature, rainfall and chlorophyll- $a$ concentrations on bud density (numbers per $\mathrm{cm}^{2}$; Chapter 4).

\begin{tabular}{|c|c|c|c|}
\hline \multicolumn{4}{|c|}{ Probability of budding } \\
\hline & $d f$ & $\mathrm{~F}$ & $P$ \\
\hline Site & 1 & 0.011 & 0.916 \\
\hline Size & 1 & 37.4319 & $<0.001$ \\
\hline Species & 1 & 26.9981 & $<0.001$ \\
\hline SST & 1 & 8.8614 & 0.003 \\
\hline Rainfall & 1 & 4.5641 & 0.033 \\
\hline Chl- $a$ & 1 & 0.1111 & 0.739 \\
\hline Residuals & 473 & & \\
\hline
\end{tabular}

Table A5f. Results from a Type-III ANOVA for effects from the best fit model for bud density. Species refers to either Tethya bergquistae or T. burtoni, site refers to either Breaker Bay or Princess Bay. Sea surface temperature (SST) in ${ }^{\circ} \mathrm{C}$ and chlorophyll- $a$ concentrations in $\mathrm{mg} / \mathrm{m}^{3}$ (Chapter 4).

\begin{tabular}{|c|c|c|c|}
\hline \multicolumn{4}{|c|}{ Probability of budding } \\
\hline & $d f$ & $\mathrm{~F}$ & $P$ \\
\hline SST & 1 & 9.3344 & 0.002 \\
\hline Rainfall & 1 & 5.4087 & 0.020 \\
\hline Size $\times$ Species & 1 & 4.1933 & 0.041 \\
\hline Residuals & 474 & & \\
\hline
\end{tabular}




\section{APPENDIX 6:}

\section{IN SITU OBSERVATIONS OF BUDDING EVENTS RECORDED FOR MONITORED SPONGES}

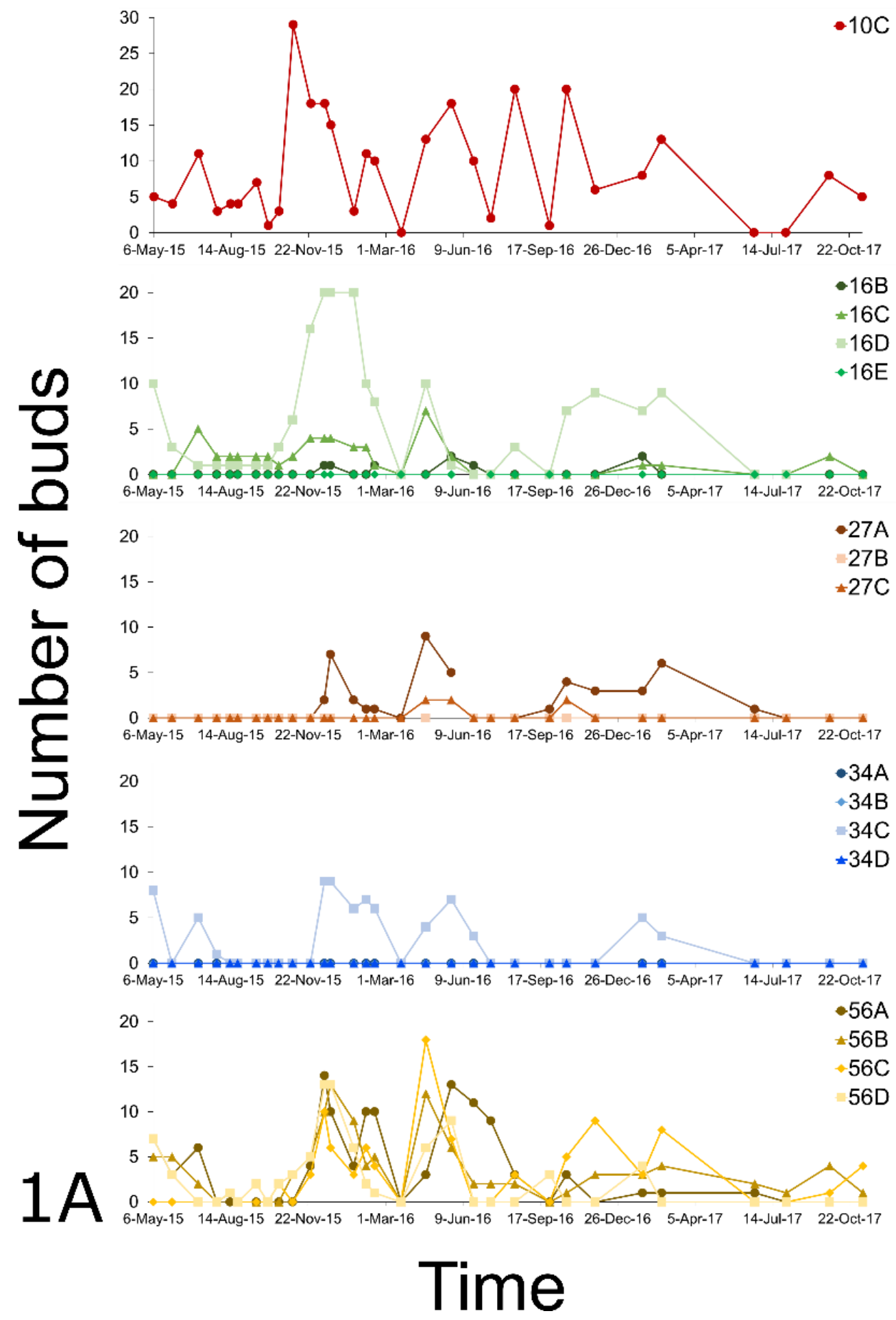

Figure A6a. Number of buds over time for each monitored Tethya bergquistae (sponge ID indicated by tag number in legend) for tagged sites. Each number (1-6) represents a site, each graph represents a tag, and each line represents a tagged sponge. 


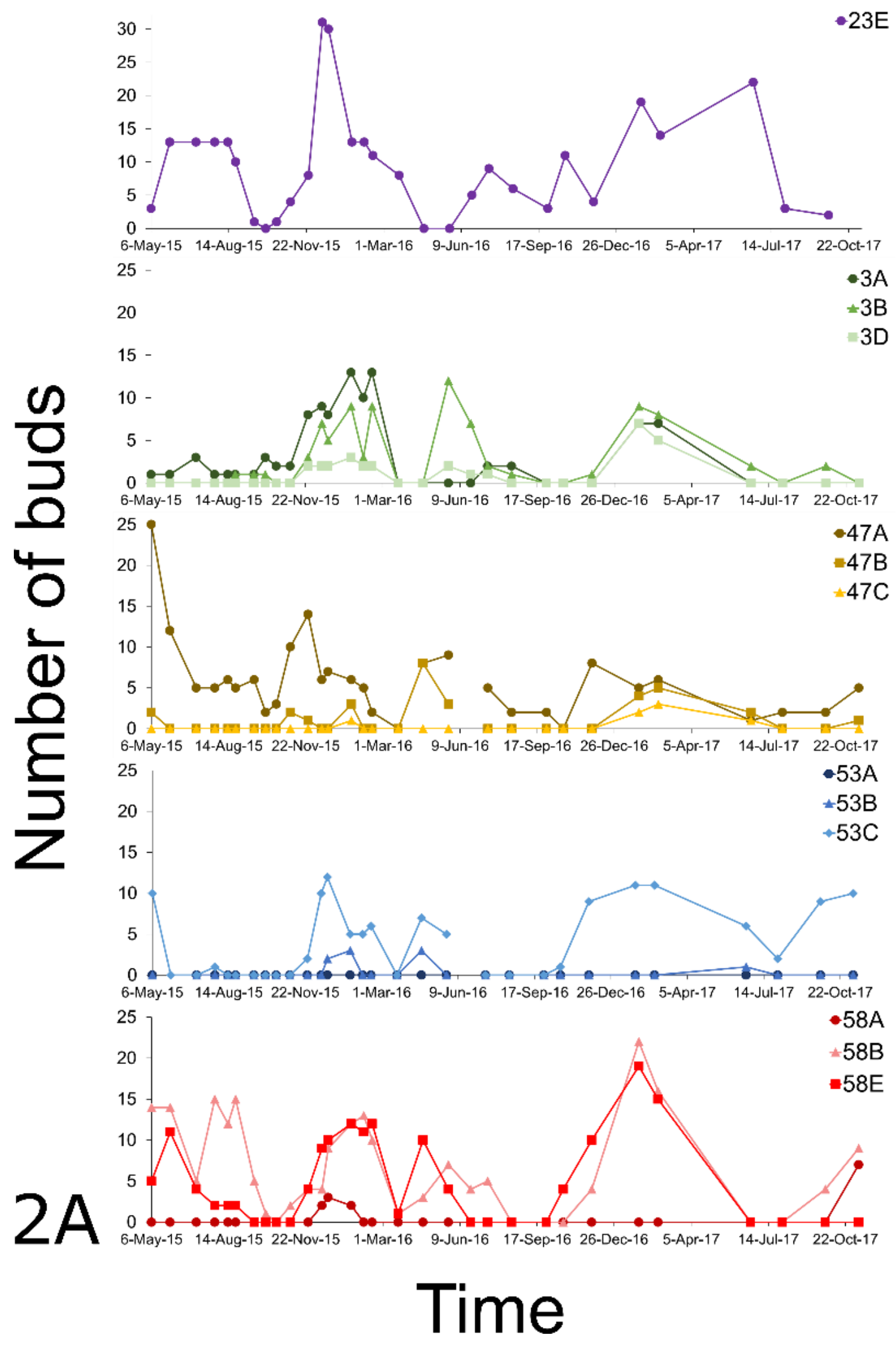

Figure A6a (contin.). Number of buds over time for each monitored Tethya bergquistae (sponge ID indicated by tag number in legend) for tagged sites. Each number (1-6) represents a site, each graph represents a tag, and each line represents a tagged sponge. 


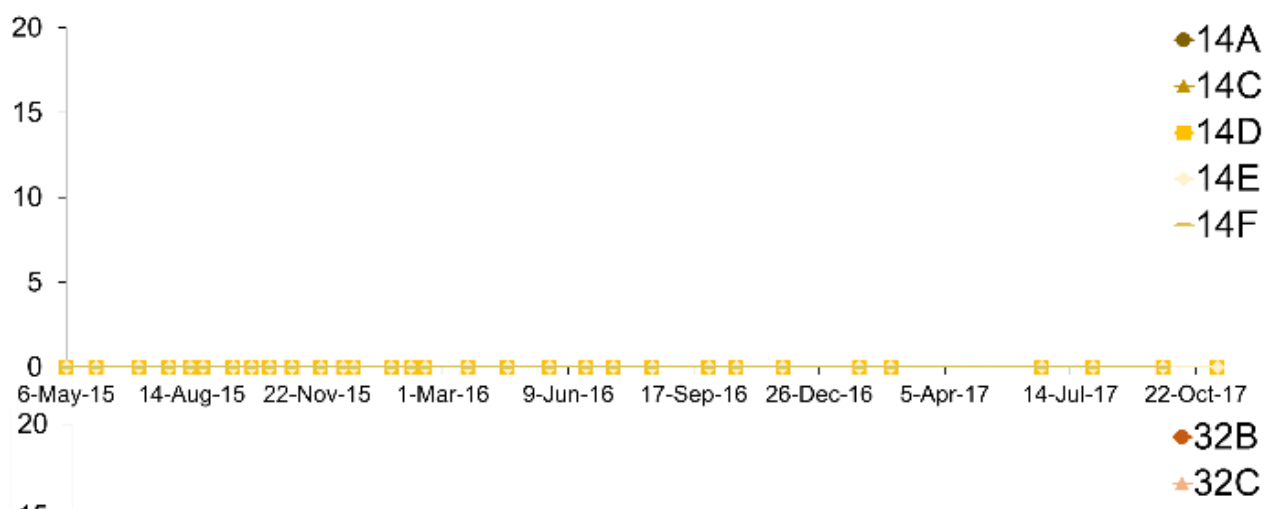

15
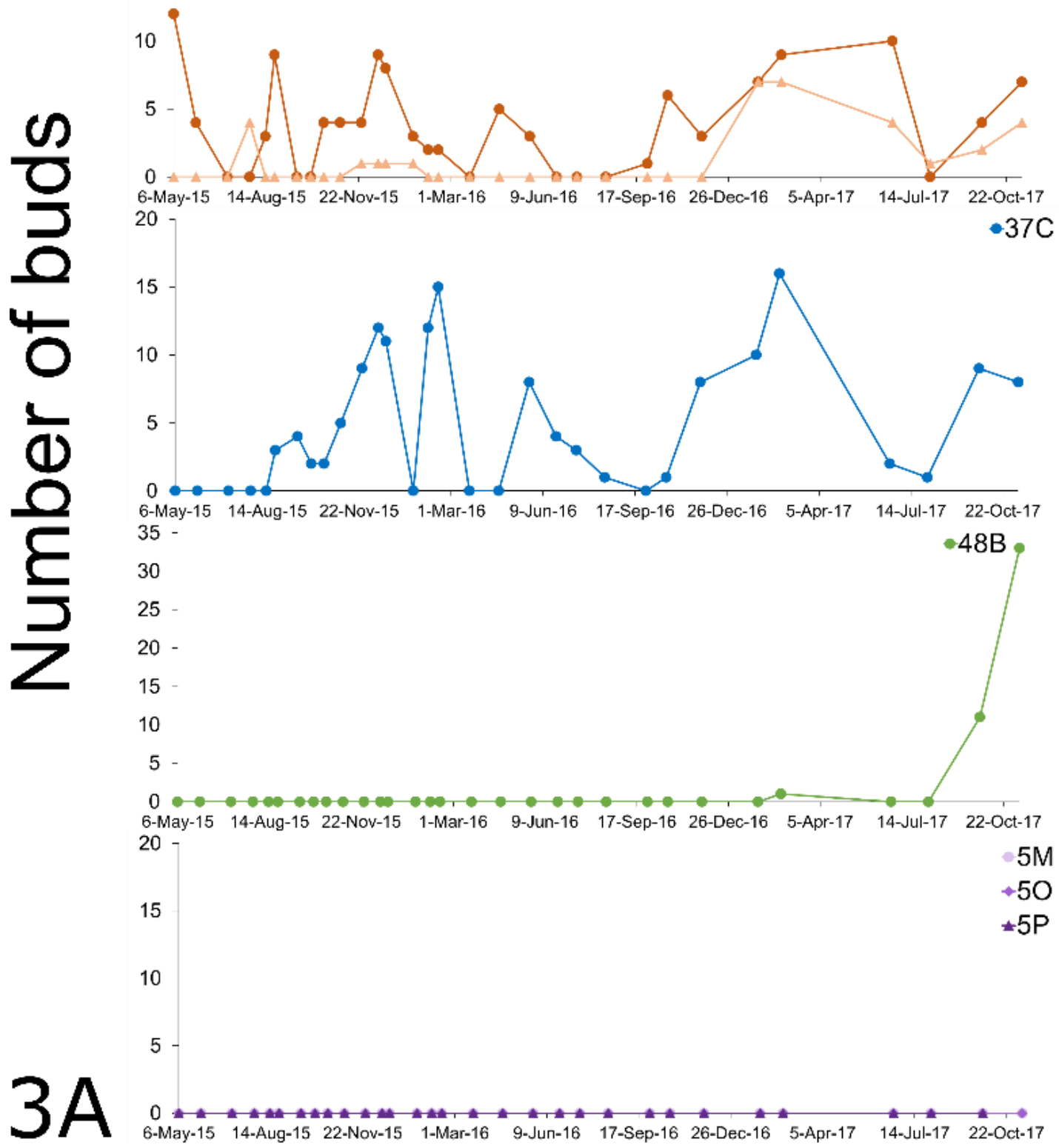

\section{Time}

Figure A6a (contin.). Number of buds over time for each monitored Tethya bergquistae (sponge ID indicated by tag number in legend) for tagged sites. Each number (1-6) represents a site, each graph represents a tag, and each line represents a tagged sponge. 


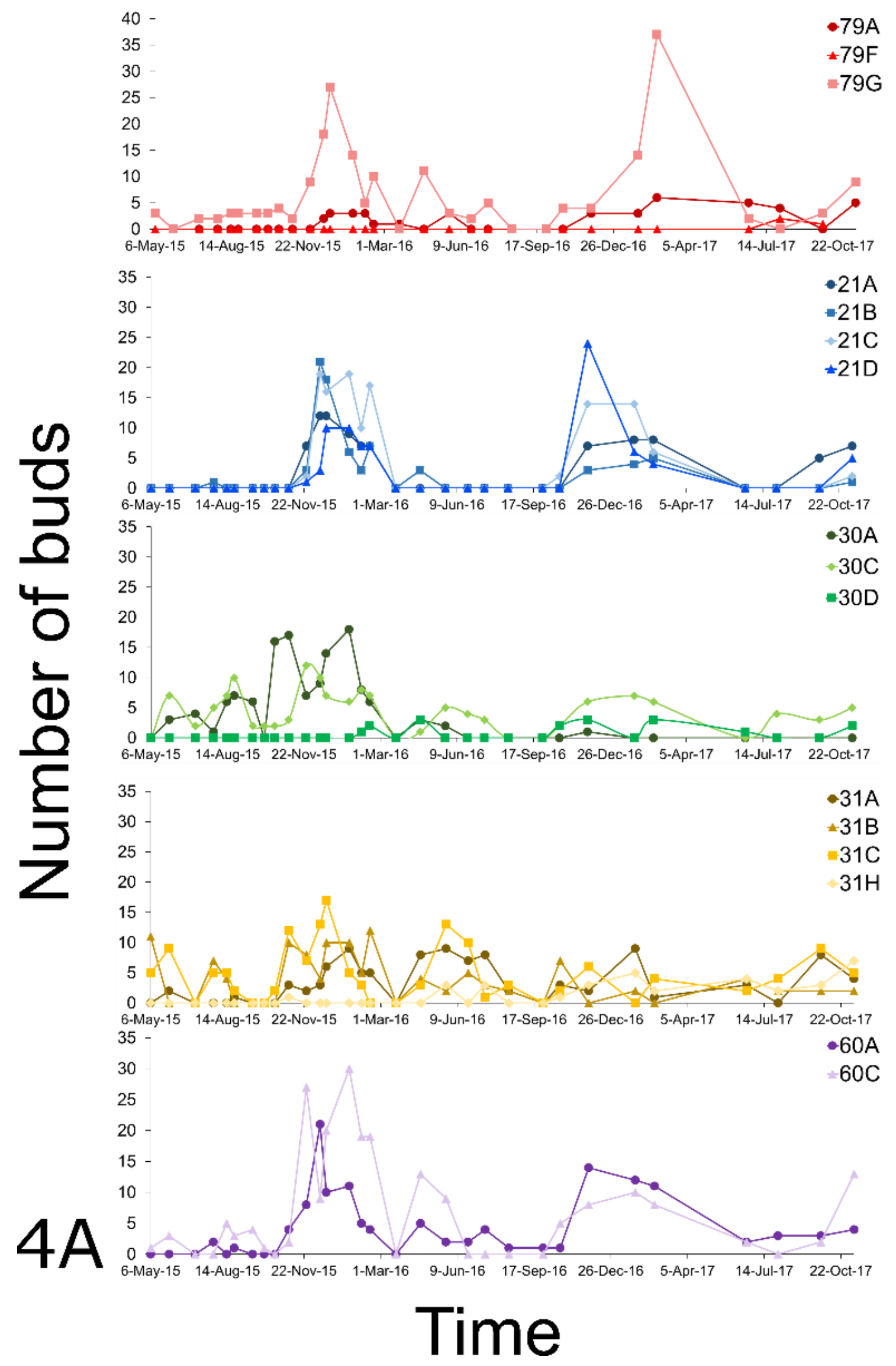

Figure A6a (contin.). Number of buds over time for each monitored Tethya bergquistae (sponge ID indicated by tag number in legend) for tagged sites. Each number (1-6) represents a site, each graph represents a tag, and each line represents a tagged sponge. 


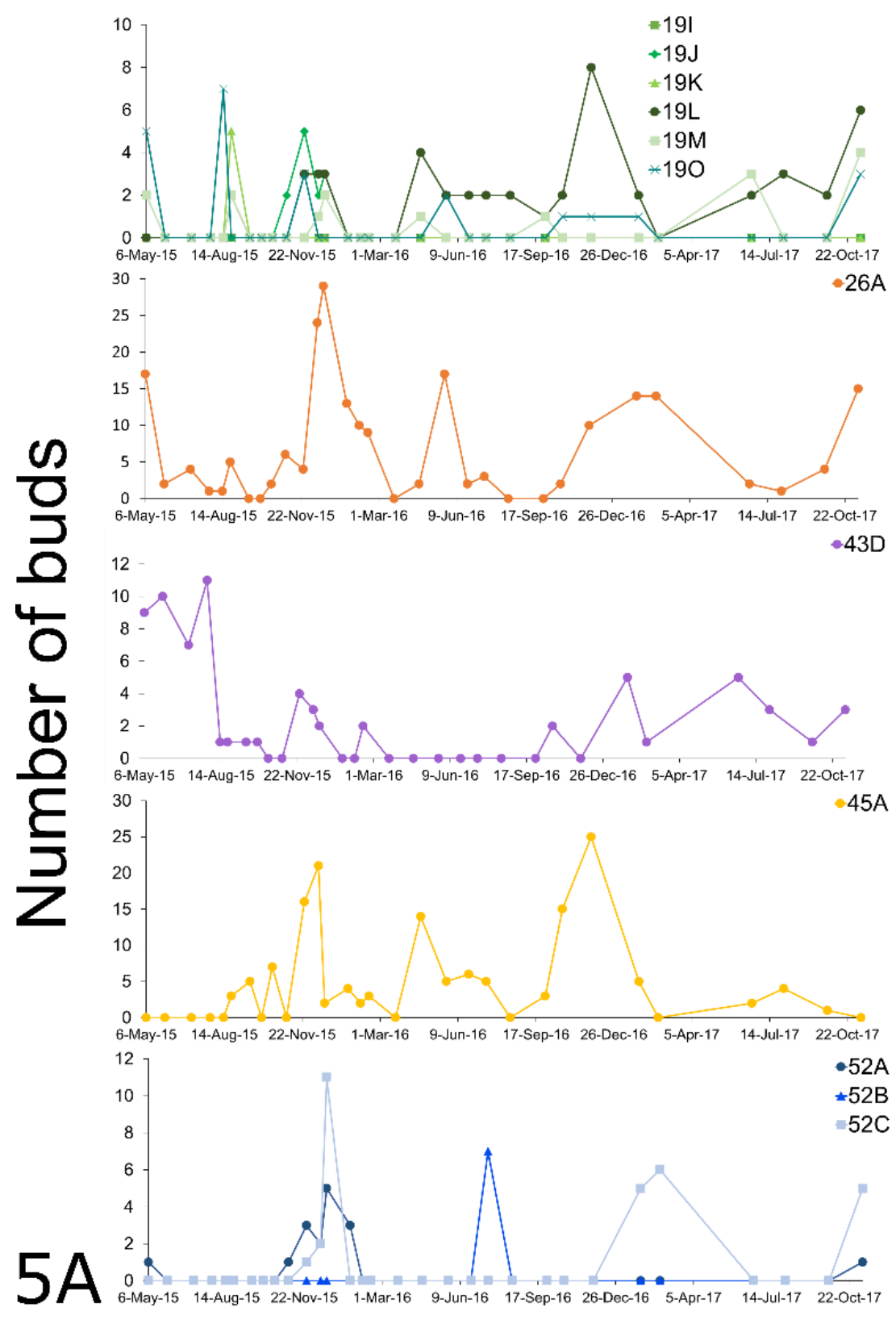

\section{Time}

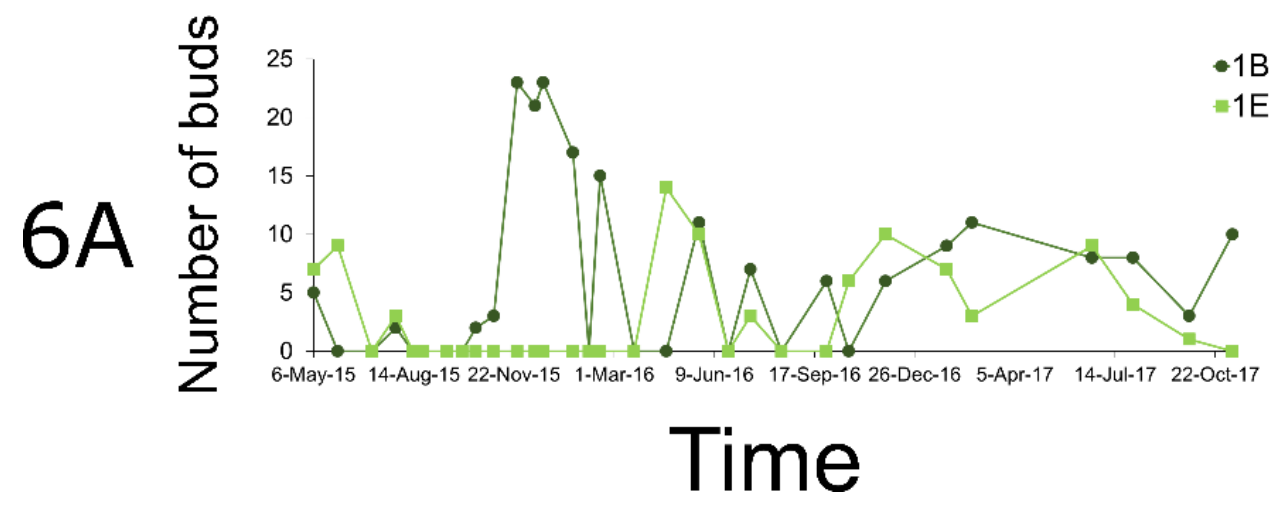

Figure A6a (contin.). Number of buds over time for each monitored Tethya bergquistae (sponge ID indicated by tag number in legend) for tagged sites. Each number (1-6) represents a site, each graph represents a tag, and each line represents a tagged sponge. 


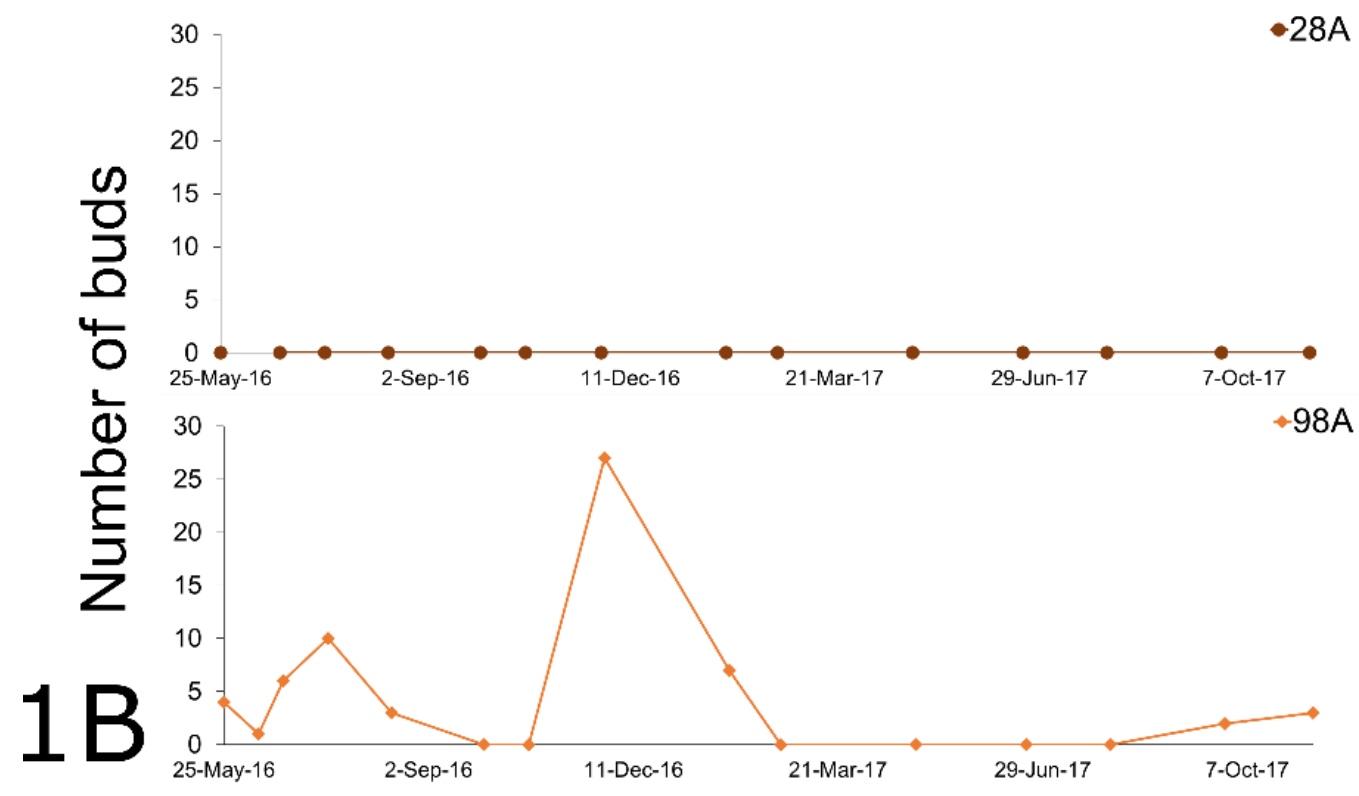

\section{Time}

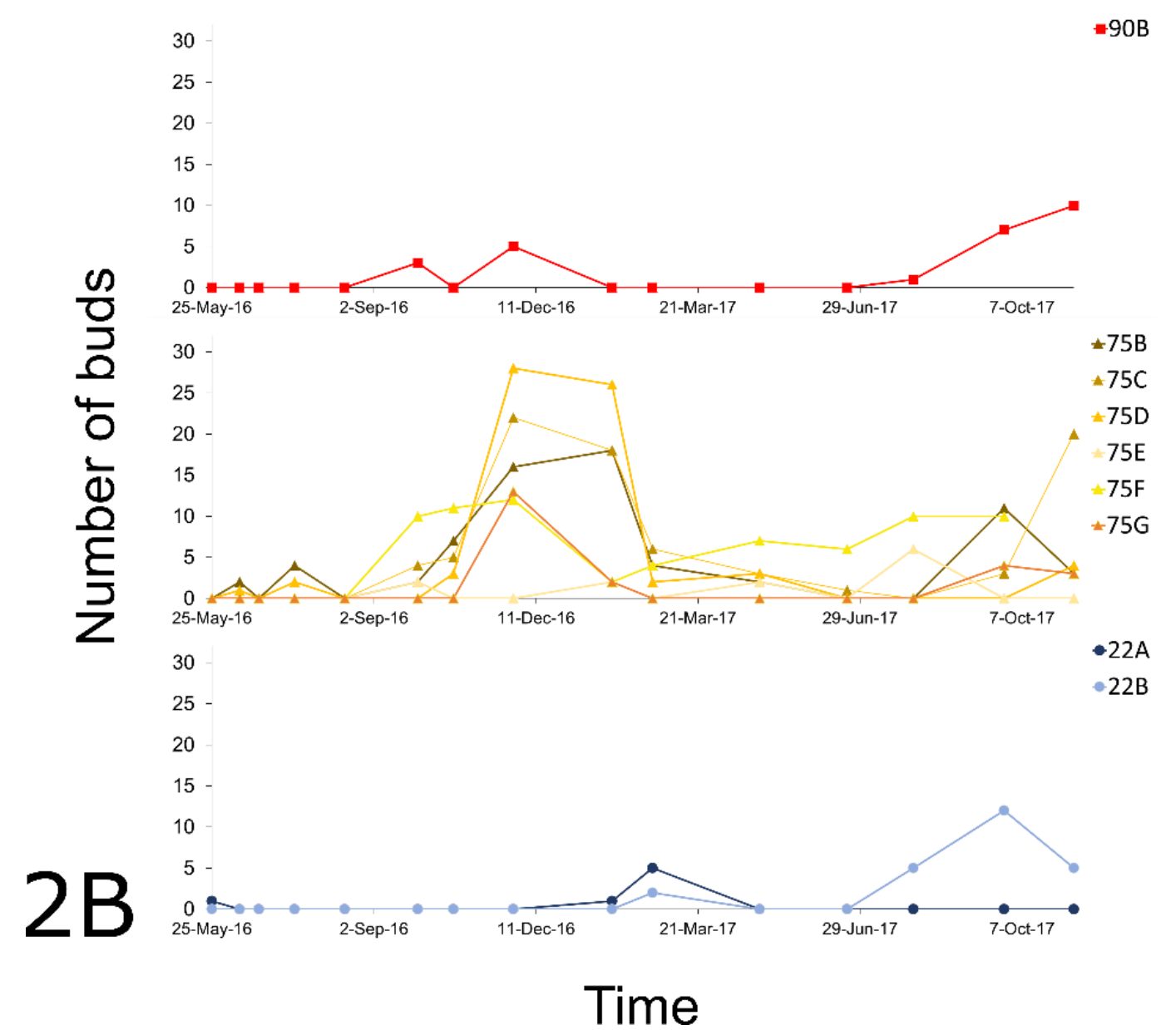

Figure A6b. Number of buds over time for each monitored Tethya burtoni (sponge ID indicated by tag number in legend) for tagged sites. Each number (1-6) represents a site, each graph represents a tag, and each line represents a tagged sponge. 


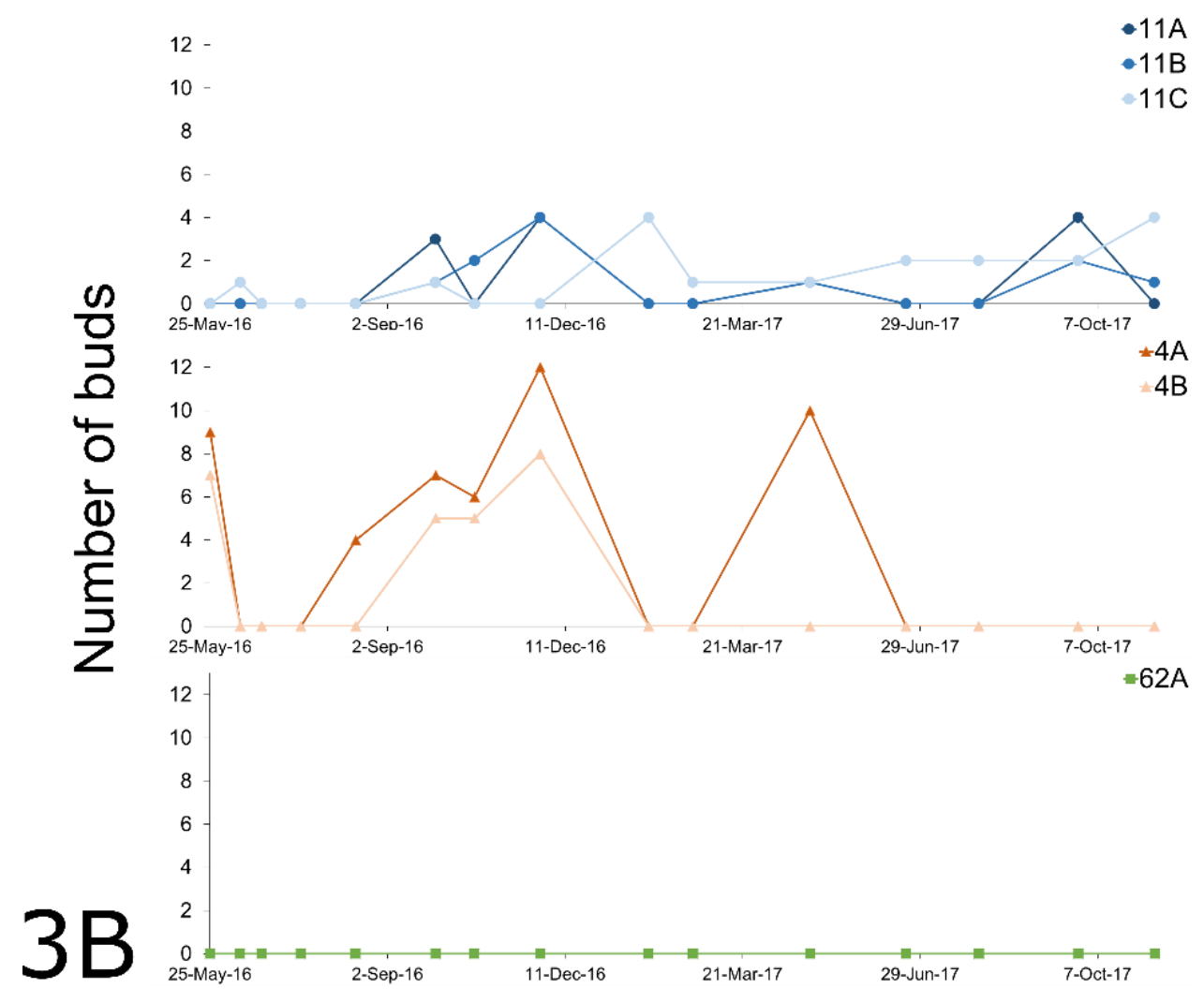

Time

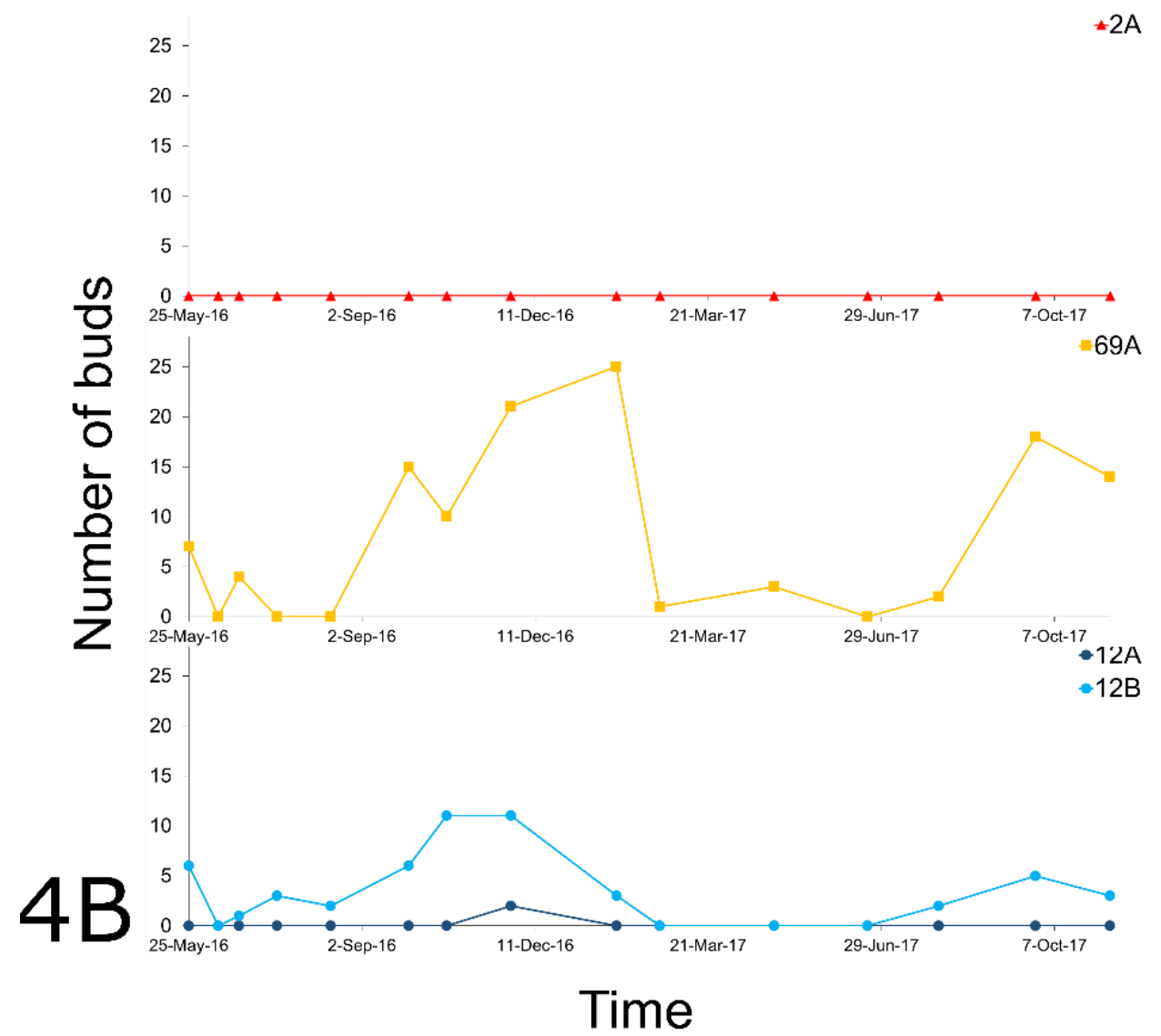

Figure A6b (contin.). Number of buds over time for each monitored Tethya burtoni (sponge ID indicated by tag number in legend) for tagged sites. Each number (1-6) represents a site, each graph represents a tag, and each line represents a tagged sponge. 

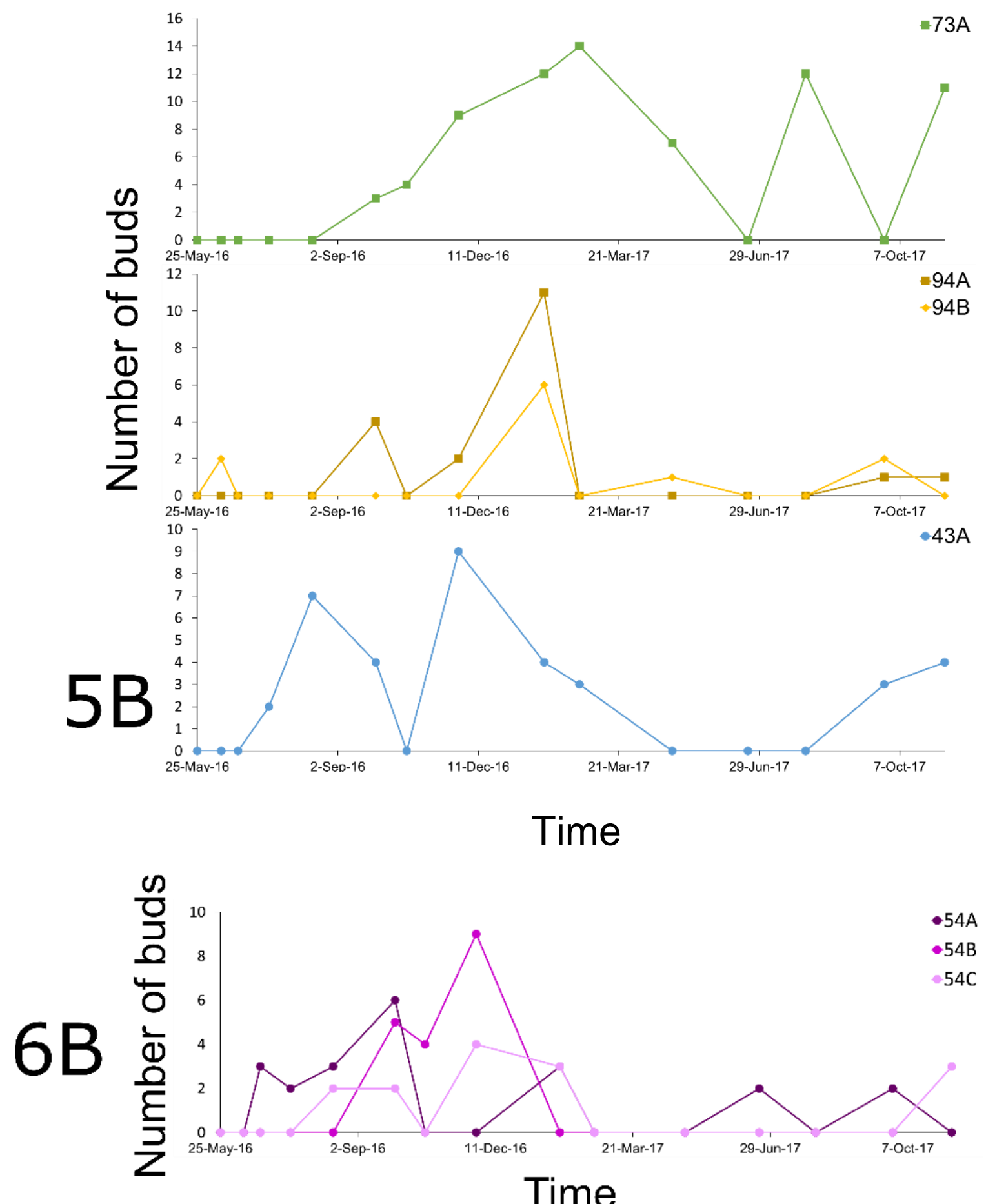

Figure A6b (contin.). Number of buds over time for each monitored Tethya burtoni (sponge ID indicated by tag number in legend) for tagged sites. Each number (1-6) represents a site, each graph represents a tag, and each line represents a tagged sponge. 


\section{APPENDIX 7: \\ INFORMATION ON CLONAL GROUPS}

Table A7. Clone groups of Tethya burtoni found at Breaker Bay, with information for clone group ID (1-4); sample ID (i.e. BB\#); gametes observed ( 0 = no gametes, 1 = gametes present, $\mathrm{O}=$ oocytes, $\mathrm{SC}=$ spermatic cysts); $\mathrm{ROI}$ (or reproductive output index, which is a measure of reproductive effort and is calculated as the percent of tissue occupied by gametes over the total tissue area); budding observed ( $0=$ no budding, $1=$ buds present); and budding period (time of budding events). Duration of the monitoring period, as well as times monitored (X mon), for each clone group are also given. Square area containing all clones is given in $\mathrm{cm}^{2}$, along with radial area (radial area around the sponge containing gametes to the point where all other clones are encompassed). Sponges in grey were mortalities over the monitoring period.

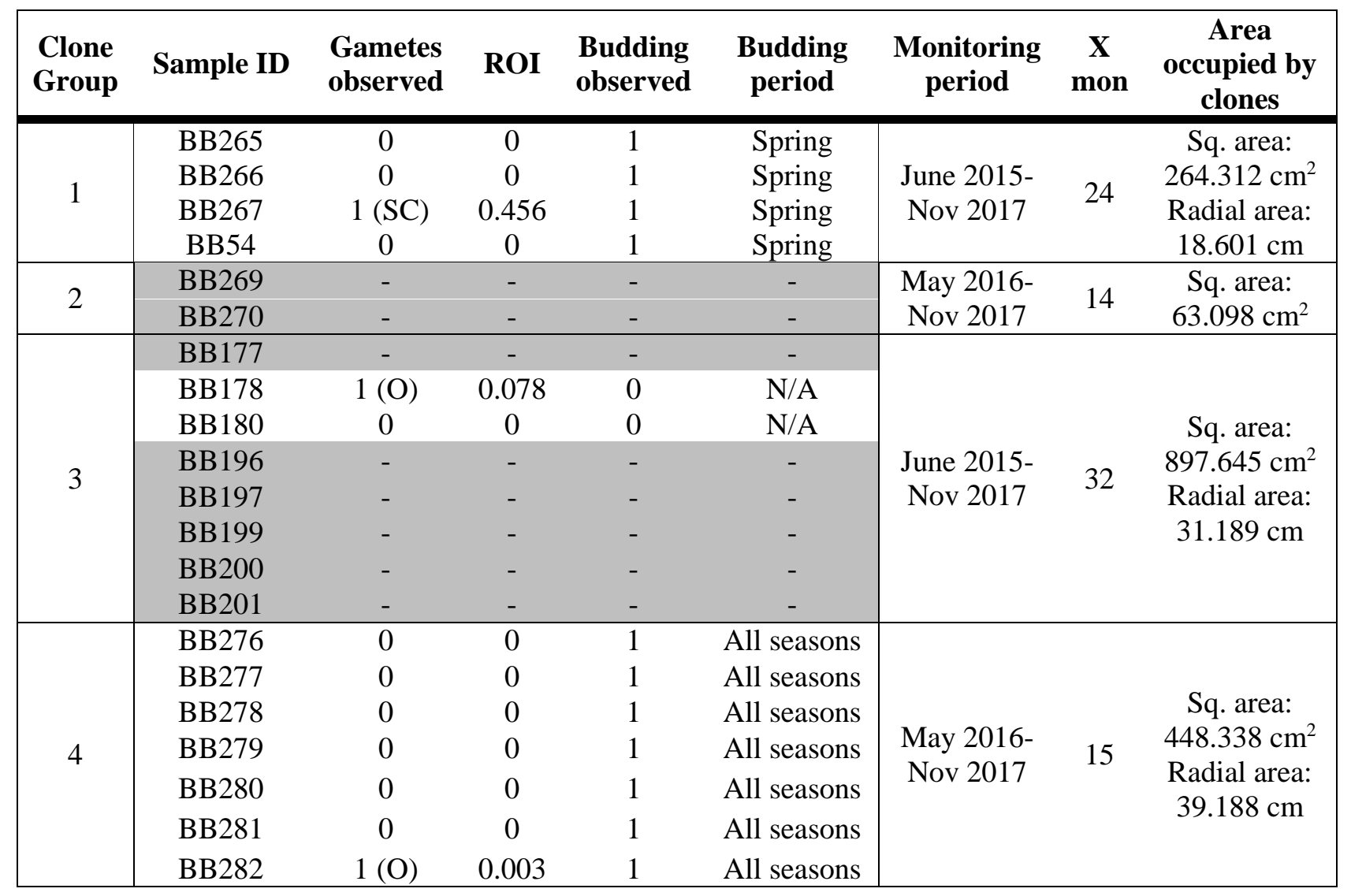




\section{APPENDIX 8:}

\section{LINKAGE DISEQUILIBRIUM BETWEEN PAIRS OF MICROSATELLITE LOCI}

Table A8. Linkage disequilibrium observed across populations of Tethya burtoni for analysis excluding clones (without clones) and including clones (with clones), for all microsatellite loci and for only those loci in HWE.

\begin{tabular}{|c|c|c|}
\hline $\begin{array}{c}\text { Linkage } \\
\text { disequilibrium }\end{array}$ & All loci & HWE loci \\
\hline Without clones & No pairs & No pairs \\
\hline With clones & $\begin{array}{l}\text { TBURT1 \& TBURT2 } \\
\text { TBURT1 \& TBURT3 } \\
\text { TBURT1 \& TBURT5 } \\
\text { TBURT1 \& TBURT10 } \\
\text { TBURT2 \& TBURT5 } \\
\text { TBURT2 \& TBURT9 } \\
\text { TBURT2 \& TBURT10 } \\
\text { TBURT3 \& TBURT6 } \\
\text { TBURT3 \& TBURT10 } \\
\text { TBURT4 \& TBURT10 } \\
\text { TBURT5 \& TBURT6 } \\
\text { TBURT5 \& TBURT9 } \\
\text { TBURT5 \& TBURT10 } \\
\text { TBURT6 \& TBURT10 } \\
\text { TBURT8 \& TBURT10 } \\
\text { TBURT9 \& TBURT10 }\end{array}$ & $\begin{array}{l}\text { TBURT1 \& TBURT2 } \\
\text { TBURT1 \& TBURT5 } \\
\text { TBURT1 \& TBURT6 } \\
\text { TBURT1 \& TBURT10 } \\
\text { TBURT2 \& TBURT5 } \\
\text { TBURT2 \& TBURT6 } \\
\text { TBURT2 \& TBURT9 } \\
\text { TBURT2 \& TBURT10 } \\
\text { TBURT4 \& TBURT10 } \\
\text { TBURT5 \& TBURT6 } \\
\text { TBURT5 \& TBURT9 } \\
\text { TBURT5 \& TBURT10 } \\
\text { TBURT6 \& TBURT9 } \\
\text { TBURT6 \& TBURT10 } \\
\text { TBURT8 \& TBURT10 } \\
\text { TBURT9 \& TBURT10 }\end{array}$ \\
\hline
\end{tabular}


APPENDIX 9:

PUBLICATION OF CHAPTER 2 


\title{
Hidden diversity in the genus Tethya: comparing molecular and morphological techniques for species identification
}

\author{
Megan R. Shaffer ${ }^{1} \cdot$ Simon K. Davy $\mathbb{( D}^{1} \cdot$ James J. Bell $\mathbb{( \mathbb { D }}^{1}$
}

Received: 25 April 2018 / Revised: 25 June 2018 / Accepted: 29 July 2018 / Published online: 21 August 2018

(c) The Genetics Society 2018

\begin{abstract}
Correctly determining species' identity is critical for estimating biodiversity and effectively managing marine populations, but is difficult for species that have few morphological traits or are highly plastic. Sponges are considered a taxonomically difficult group because they lack multiple consistent diagnostic features, which coupled with their common phenotypic plasticity, makes the presence of species complexes likely, but difficult to detect. Here, we investigated the evolutionary relationship of Tethya spp. in central New Zealand using both molecular and morphological techniques to highlight the potential for cryptic speciation in sponges. Phylogenetic reconstructions based on two mitochondrial markers (rnl, COI-ext) and one nuclear marker (18S) revealed three genetic clades, with one clade representing Tethya bergquistae and two clades belonging to what was a priori thought to be a single species, Tethya burtoni. Eleven microsatellite markers were also used to further resolve the T. burtoni group, revealing a division consistent with the $18 \mathrm{~S}$ and $\mathrm{rnl}$ data. Morphological analysis based on spicule characteristics allowed $T$. bergquistae to be distinguished from $T$. burtoni, but revealed no apparent differences between the $T$. burtoni clades. Here, we highlight hidden genetic diversity within $T$. burtoni, likely representing a group consisting of incipient species that have undergone speciation but have yet to express clear morphological differences. Our study supports the notion that cryptic speciation in sponges may go undetected and diversity underestimated when using only morphology-based taxonomy, which has broad scale implications for conservation and management of marine systems.
\end{abstract}

\section{Introduction}

Correctly identifying species units is critical for estimating biodiversity, defining species boundaries and measuring connectivity patterns (Hey et al. 2003; Frankham et al. 2012). Methods for species identification have been so hotly debated in the literature that the phrase 'species problem' has emerged to describe the conundrum that biologists face when defining a species (Mayden 1997; De Queiroz 2007). Mayr (1942) was the first to address this problem by conceiving the biological species concept, where he defined a species as a group of interbreeding individuals which are

Electronic supplementary material The online version of this article (https://doi.org/10.1038/s41437-018-0134-6) contains supplementary material, which is available to authorized users.

Megan R. Shaffer

Megan.Shaffer@vuw.ac.nz

1 School of Biological Sciences, Victoria University of Wellington, Wellington 6140, New Zealand reproductively isolated from other groups. Since then, other concepts have emerged based on: an organism's functional role/ecological niche (ecological species concept, Van Valen 1976); historical ancestry lineages (evolutionary species concept, Simpson 1951); and the sharing of common ancestors/alleles (phylogenetic species concept, Hennig 1966; Rosen 1979; Baum and Shaw 1995; reviewed in De Queiroz 2007). While many concepts exist, the most traditional, widespread and consistently employed method for taxonomy is based on comparative morphology (Padial et al. 2010).

The recent advancement and accessibility of molecular tools has begun to reveal conflicting evolutionary histories for many species previously defined by morphological characteristics (Wendel and Doyle 1998; Knowlton 2000; Lecompte et al. 2005; Lihová et al. 2007; Wallis et al. 2017). Distinguishing between intraspecific plasticity and interspecific cryptic diversity can be problematic when only using morphology-based taxonomic methods (Knowlton 1993; Vrijenhoek 2009; Capa et al. 2013, Czarna et al. 2016). Additionally, congeneric species that live in sympatry often challenge the notion of species boundaries 

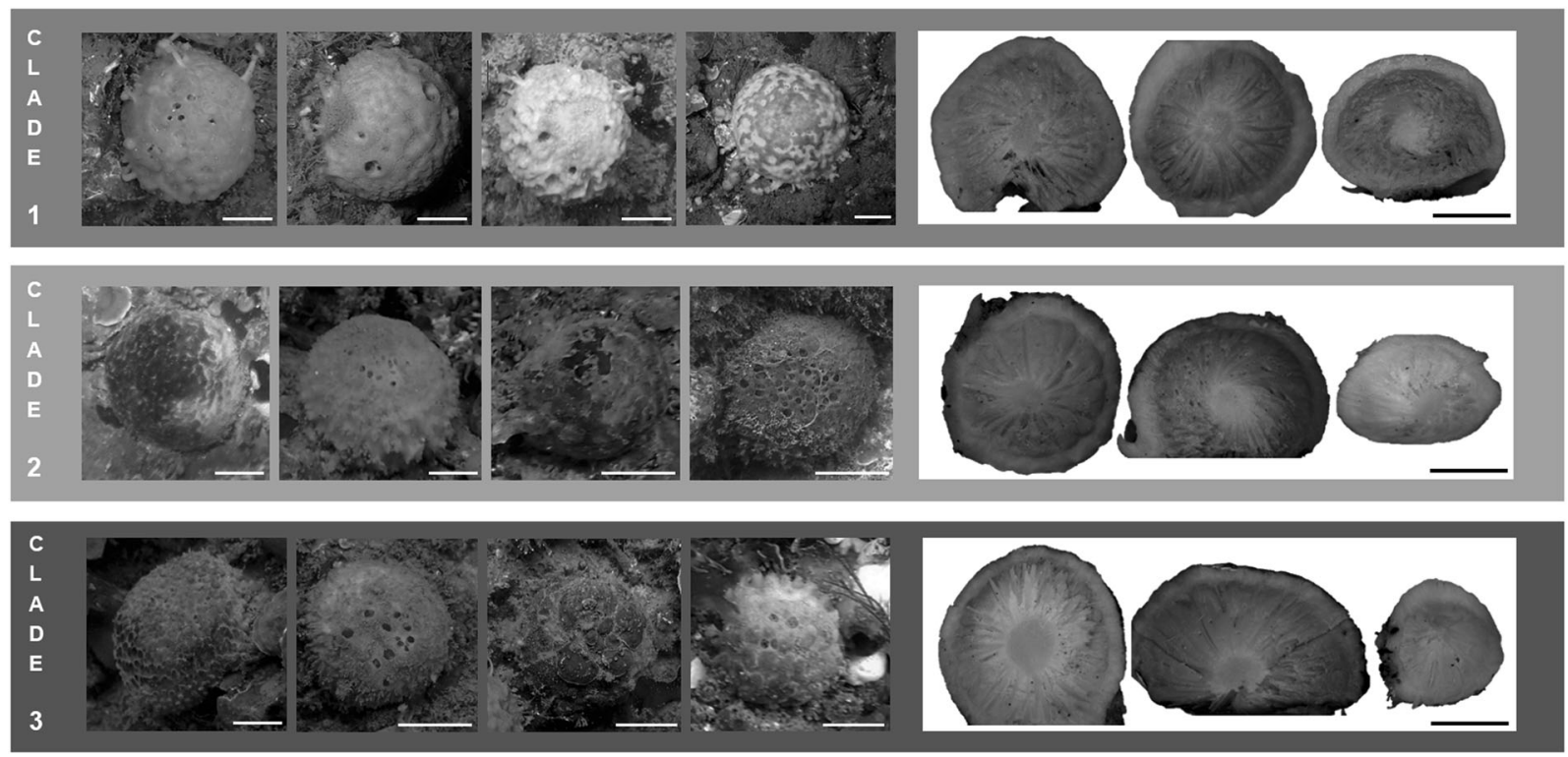

Fig. 1 Examples of sponges collected belonging to clades identified in Fig. 3, to allow comparison of macromorphological features. For each clade, cross sections that encompass the ectosome and choanosome are included for a subsample $(n=3)$ of genetically identical individuals.

because introgression (i.e. where alleles from one species enter the gene pool of another species via hybridization and backcrossing) is common (Harrison and Larson 2014). Failure to correctly identify species boundaries has consequences for implementing effective conservation and management strategies and for our overall understanding of important evolutionary processes like speciation (Gómez et al. 2002), bioinvasions (Knapp et al. 2015) and hybridization (Kauserud et al. 2006).

Organisms that have only a few defining traits available for comparison are particularly prone to identification problems (Knowlton 1993; Bickford et al. 2007; DeBiasse and Hellberg 2015). In such groups, cryptic species often emerge during molecular studies, as sequences provide more characteristics (bases) with which to differentiate organisms, which allows a finer resolution of relatedness between individuals to be determined (Hilis 1987; Hebet et al. 2004; Bickford et al. 2007). Sponges have relatively few morphological traits, yet a rich taxonomic literature exists delineating species boundaries within sponges based on phenotypic characteristics (Hooper and Van Soest 2002). Skeletal composition, arrangement and spicule morphology are key features used to classify and identify sponges (Hooper and Van Soest 2002), despite the fact that structural components of sponges can be highly dynamic (Bond 1992; Nakayama et al. 2015) and spicules can exhibit variability under different environmental conditions (Bavestrello et al. 1993; Bell et al. 2002; McDonald et al. 2002). Furthermore, recent studies on sponge taxonomy
Clade 1 (top) are examples of Tethya bergquistae, while Clades 2 and 3 (middle and bottom, respectively) are examples of $T$. burtoni. Scale bars in each photo $=10 \mathrm{~mm}$

have highlighted problems with using morphology as a sole defining trait and have used phenotypic traits combined with molecular analyses to resolve evolutionary relationships (Alvarez et al. 2000; Erwin and Thacker 2007; Morrow and Cárdenas 2015; Plotkin et al. 2017). As a result, phylogenetic analyses for many sponges have begun to reveal cryptic speciation throughout the phylum, suggesting that diversity within the phylum is even higher than previously thought (see Table 1 in Xavier et al. (2010) for reported cases of cryptic speciation in sponges, plus Andreakis et al. 2012; De Paula et al. 2012; DeBiasse and Hellberg 2015; Knapp et al. 2015; Uriz et al. 2017).

In this study, we used 'golf ball sponges' belonging to the genus Tethya in New Zealand as a model to investigate cryptic speciation and to better understand how evolutionary processes occur for species living in sympatry. Tethya is a ubiquitous genus, with 92 described species worldwide (Van Soest 2008), and has a particularly high diversity (31 species) recorded in coastal waters of Australia and New Zealand (Bergquist and Kelly-Borges 1991; Sarà 1998; Sarà and Sarà 2004; Heim et al. 2007). While members of the genus are easily distinguishable by their globular form, identifying Tethya to the species level can be difficult because they exhibit a high degree of morphological plasticity, and have only a few defining traits available for species differentiation. The challenge in species identification is reflected in the dynamic taxonomic literature, where new species are constantly being described (Corriero et al. 2015), and previously described species are regularly 
reconsidered and reclassified (Sarà 1987; Bergquist and Kelly-Borges 1991; Hooper and Wiedenmayer 1994; Sarà and Sarà 2004; Heim et al. 2007). In New Zealand, taxonomy has been based solely on morphology with no genetic phylogenies constructed to date (Bergquist and Kelly-Borges 1991; Sarà and Sarà 2004). Here, we used two common species of Tethya in New Zealand (Tethya bergquistae and Tethya burtoni) as models to investigate evolutionary relationships and cryptic speciation using both genetic (COI-ext, rnl, $18 \mathrm{~S}$ and 11 microsatellite markers) and morphological traits (spicules) in order to understand the advantages and limitations of both methods in determining species boundaries.

\section{Materials and methods}

\section{Study species}

Tethya bergquistae and $T$. burtoni are two of the most common species of Tethya found in the waters of central New Zealand, and both occur in the waters around Wellington, New Zealand (Fig. 1). Tethya bergquistae is described as rose-pink in colour, firm to the touch and with an irregular surface with grouped oscula (Bergquist and Kelly-Borges 1991; Hooper and Wiedenmayer 1994; Battershill et al. 2010). Tethya burtoni is bright orange to yellow and is described as having a warty surface with an inflated, soft texture (Sarà and Sarà 2004; Battershill et al. 2010). Tethya bergquistae is generally found in well-lit areas with moderate flow, whereas $T$. burtoni tends to prefer more shaded, sedimented areas (Battershill et al. 2010). Spicules for both include megascleres, which are strongyloxeas; and microscleres, which are megasters and micrasters of similar sizes (Bergquist and Kelly-Borges 1991; Sarà and Sarà 2004). A key distinguishing feature between both species relies on their spicular composition with respect to the location/density of megasters, as $T$. burtoni contains fewer megasters, which are mainly found in the cortex (Bergquist and Kelly-Borges 1991; Sarà and Sarà 2004).

Tracking the description of both species through the literature reveals a complex taxonomic classification history within New Zealand, highlighting the complexity in species identification common to Porifera. For instance, T. bergquistae was incorrectly identified as Tethya ingalli by Pritchard et al. (1984) and formerly described as Tethya australis by Bergquist and Kelly-Borges (1991), and T. burtoni has been also incorrectly referred to as Tethya aurantium by Pritchard et al. (1984). Overall in New Zealand, there are ten species of Tethya recorded, but two of these are also thought to exist in Australian waters
(Ledenfield 1888; Bergquist 1961; Bergquist and KellyBorges 1991; Sarà and Sarà 2004). Many of these ten species are thought to be endemic to certain regions (e.g. Tethya compacta to the Chatham Islands and Tethya fastigata to the Poor Knight Islands), but this has been based on observations from only one to a few sponges (e.g. $T$. compacta), or on descriptions dating from the 19th Century (e.g. Tethya multistella). In the literature, pictures identifying New Zealand Tethya are often inconsistent and classification by comparative morphology is therefore challenging. Descriptions of species within Tethya are based on cross sections and spicule compliments, but differences can be often subtle and difficult to detect, especially for non-specialists. Around New Zealand, sympatric Tethya spp. display a wide range of colours and textures, making them difficult to confidently match to descriptions in the current taxonomic literature. Tethya also appear to undergo seasonal morphological changes (M. Shaffer, personal observations from monthly monitoring for $2+$ years). For example, the same sponge's appearance may go from porous and soft to very firm over a period of a month. In addition, during the summer a sponge may be covered more extensively by crustose coralline algae (CCA), rendering it more reminiscent of Tethya amplexa (Bergquist and KellyBorges 1991), but in the winter be completely free of CCA. Because morphology can be very variable and temporally dependent, assessing species based solely on morphology has the potential to lead to erroneous conclusions. To date, molecular differences have allowed at least one other instance of sibling species within the genus Tethya to be uncovered. Sarà et al. (1993) reported cryptic speciation in two populations of Tethya robusta, which were morphologically similar but genetically different. The taxonomic history, phenotypic plasticity and previous report of a species complex within the Tethya genus makes other occurrences of cryptic speciation within this group likely.

\section{Collection}

Sponges which were a priori thought to be $T$. bergquistae and $T$. burtoni were collected using SCUBA from two different locations in waters around Wellington, New

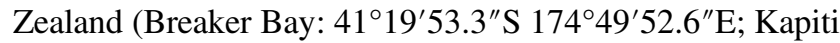
Island: $40^{\circ} 53^{\prime} 23.6^{\prime \prime} \mathrm{S} 174^{\circ} 52^{\prime} 40.3^{\prime \prime} \mathrm{E}$ ). Additional specimens of T. burtoni were collected from two further locations (Somes Island: $41^{\circ} 15^{\prime} 36.9^{\prime \prime} \mathrm{S} 174^{\circ} 43^{\prime} 54.4^{\prime \prime} \mathrm{E}$; Red Rocks: $\left.41^{\circ} 21^{\prime} 04.5^{\prime \prime} \mathrm{S} 174^{\circ} 43^{\prime} 54.4^{\prime \prime} \mathrm{E}\right)$. Sponges were collected $3-5 \mathrm{~m}$ apart to avoid collecting clones, at depths from 5 to $10 \mathrm{~m}$. Three sponges of unknown identity were collected from Breaker Bay $(n=2)$ and Kapiti Island $(n=1)$, which were texturally similar to $T$. bergquistae but the same colour as T. burtoni. A picture of each specimen was taken 
Table 1 Primer information for markers used: $18 \mathrm{~S}$, rnl and COIext. Forward and reverse sequences presented $5^{\prime} \rightarrow 3^{\prime}$. Product sizes are in base pairs. Accession numbers refer to distinct haplotypes and genotypes that were deposited to GenBank for each marker.

\begin{tabular}{lllll}
\hline Region & Primers & $\begin{array}{l}\text { Size } \\
(\mathrm{bp})\end{array}$ & Ref. & $\begin{array}{l}\text { Accession number of } \\
\text { products }\end{array}$ \\
\hline 18S & $\begin{array}{l}\text { 18S_1-600_F: } \\
\text { GCCAGTAGTCATATGCTTGTCTCA }\end{array}$ & 425 & $\begin{array}{l}\text { Knapp et al. } \\
2015\end{array}$ & MH180010-MH180012 \\
& $\begin{array}{l}\text { 18S_1-600_R: } \\
\text { GACTTGCCCTCCAATTGTTC }\end{array}$ & & & \\
rnl & $\begin{array}{l}\text { diplo-rnl-f1: } \\
\text { TCGACTGTTACCAAAAACATAGC }\end{array}$ & & Lavrov et al. & MH180013-MH180019 \\
& $\begin{array}{l}\text { diplo-rnl-r1: } \\
\text { AATTCAACATCGAGGTSGGAAAC }\end{array}$ & & & \\
COI-ext & COX1-R1: & 425 & Rot et al. 2006 & MH180020-MH180023 \\
& TGTTGRGGGAAAAARGTTAAATT & & & \\
& COX1-D2: & & \\
& AATACTGCTTTTTTGATCCTGCCGG & & & \\
\hline
\end{tabular}

to aid in later identification. Tissue was extracted for both genetic and spicule analysis, and the rest of the specimen was preserved in ethanol. In total, 71 sponges of various morphologies were collected for phylogenetic and morphological analyses. For those sponges assumed to be $T$. bergquistae, samples were collected from Breaker Bay $(n$ $=10)$ and Kapiti Island $(n=5)$. For those sponges assumed to be $T$. burtoni, samples were collected from Breaker Bay $(n=17)$, Kapiti Island $(n=20)$, Red Rocks $(n=5)$ and Somes Island $(n=12)$. For information on specimens collected for this study, see Table S1 in the Supplementary Information.

\section{DNA extraction, PCR amplification, sequencing and fragment analysis}

Tissue used for genetic analysis was taken from the inside (choanosome) of the sponge and rinsed thoroughly to reduce the risk of contamination from epibionts or other associated organisms. DNA was extracted using a DNeasy Blood \& Tissue Kit (Qiagen), following the protocol of the manufacturer. We tried five primer sets, but two regions failed to consistently give readable sequences across all samples (ITS, primer set: ITS2.2/ITSRA2, as in Adlard and Lester 1995; and COI, primer set: dgLCO1490/ dgHCO2198; Folmer et al. 1994), so were discarded for further analysis. Three markers were amplified in total: $18 \mathrm{~S}$, rnl and COI-ext. 18S is a nuclear gene that codes ribosomal DNA (rDNA), and $\mathrm{rnl}$ is a mitochondrial partition that codes the large ribosomal subunit RNA (Boore 1999). COIext is a region downstream of COI, which is a mitochondrial coding region for the cytochrome $c$ oxidase subunit used in cellular metabolism (Folmer et al. 1994). This extended region is thought to be more informative for closely related species because its substitution pattern (mainly consisting of transversions) reveals a more progressive stage of character evolution (Erpenbeck et al. 2006a). As such, this region exhibits variation during early stages of species divergence compared to the traditionally conserved COI region (by Folmer et al. 1994) that is commonly used (Erpenbeck et al. 2006a). Primer information and product sizes are shown in Table 1.

Reaction and cycling conditions were similar for all three markers. Reactions were carried out in $30 \mu \mathrm{l}$ volumes consisting of: $15 \mu \mathrm{l}$ MyTaq Red Mix (Bioline), $0.6 \mu \mathrm{l}$ of each primer $(10 \mu \mathrm{M}), \sim 25 \mathrm{ng}$ template DNA and a volume of distilled water to reach $30 \mu \mathrm{l}$. Amplification profiles were as follows: an initial denaturation at $94{ }^{\circ} \mathrm{C}$ for $5 \mathrm{~min}$; 35 cycles of $94{ }^{\circ} \mathrm{C}$ for $30 \mathrm{~s}, T_{\mathrm{a}}$ for $55 \mathrm{~s}\left(T_{\mathrm{a}}=50{ }^{\circ} \mathrm{C}\right.$ for $18 \mathrm{~S} ; T_{\mathrm{a}}=58^{\circ} \mathrm{C}$ for COI-ext and rnl), $72{ }^{\circ} \mathrm{C}$ for $45 \mathrm{~s}$; followed by a final extension at $72{ }^{\circ} \mathrm{C}$ for $7 \mathrm{~min}$. Products were visually checked on a $1.5 \%$ agarose gel stained with RedSafe Nucleic Acid Staining Solution (20,000×) and sequenced using automated DNA sequencing services provided by Macrogen, Inc. (Seoul, South Korea). Sequences were deposited into GenBank, with accession numbers for haplotypes of all individuals provided in Table S1 in the Supplementary Information.

Eleven microsatellite markers were developed for $T$. burtoni (see Supplementary Information for microsatellite development and Table S2 for microsatellite information). These markers failed to amplify for $T$. bergquistae. Fragment analysis was performed on sponges belonging to the T. burtoni clades. Reactions were carried out in $30 \mu \mathrm{l}$ reactions, with $15 \mu \mathrm{l}$ MyTaq Red Mix (Bioline), $0.6 \mu \mathrm{l}$ of each primer $(10 \mu \mathrm{M}), \sim 25 \mathrm{ng}$ template DNA and a volume of distilled water to reach $30 \mu \mathrm{l}$. Amplification profiles were as follows: an initial denaturation at $94{ }^{\circ} \mathrm{C}$ for $10 \mathrm{~min}$; 35 cycles of $94{ }^{\circ} \mathrm{C}$ for $45 \mathrm{~s}, 60^{\circ} \mathrm{C}$ for $55 \mathrm{~s}, 72^{\circ} \mathrm{C}$ for $60 \mathrm{~s}$; followed by a final extension at $72{ }^{\circ} \mathrm{C}$ for $10 \mathrm{~min}$. Fluorescently labelled products were visually checked, pooled, purified and then genotyped using the 3730xl DNA Analyzer (Applied Biosystems) from GeneScan Services for fragment analysis by Macrogen Inc. (Seoul, South Korea). 
Alleles were visualized in GeneMarker v2.2 (Hulce et al. 2011) and scored manually relative to a size standard (LIZ500). Some sponges that were thought to be T. burtoni had a lower amplification success for some loci, even after redoing the DNA extraction and fragment analysis two to three times. Because of this, missing information was considered informative in analyses.

\section{Genetic analysis}

All sequences were checked for contaminants and verified as being poriferan using BLAST (http://blast.ncbi.nlm.nih. gov/Blast.cgi). Sequences were edited in Geneious 11.0.2 (Kearse et al. 2012) and aligned using CLUSTALW2.0 (Larkin et al. 2007), as implemented in Geneious 11.0.2 (Kearse et al. 2012). Phylogenetic analyses were applied to $18 \mathrm{~S}$, rnl and COI-ext separately, as well as to the mitochondrial partitions concatenated $(\mathrm{COI}-\mathrm{ext}+\mathrm{rnl})$, creating four data sets. MrModeltest v2.3 (Nylander 2004) was used to select the best-fitting model of nucleotide substitution for each data set under the Akaike information criterion, and it was determined that the SYM + G model was the best fitting model for all data sets. The closely related Tethya actinia was chosen as an outgroup because both the $18 \mathrm{~S}$ region and its complete mitochondrion genome have been sequenced and made available on GenBank, making it an ideal candidate to root all three markers (accession numbers: AY320033 for rnl and COI-ext; AY878079 for 18S).

Phylogenetic trees were constructed using both Maximum Likelihood (ML) and Bayesian Inference (BI) methods for each data set. ML constructions were performed in PAUP* 4.0 (Swofford 2002), and BI constructions were performed in MrBayes 3.2.6 (Ronquist et al. 2012), using the model criterion generated from MrModeltest v2.3 (Nylander 2004). For ML analyses, a bootstrap analysis using a tree-bisection-reconnection (TBR) heuristic search with 1000 replicates was performed to determine topological confidence of the tree. For BI analyses, posterior probability of trees was estimated using Monte Carlo Markov Chain (MCMC) analyses, where one million generations were run sampling four chains every 100 generations, with a burn-in of 0.25 . For each marker, $p$ distances were calculated within and between clades as a measure of divergence using MEGA 7 (Kumar et al. 2015). In addition, $p$ distances were calculated between and within T. bergquistae (Clade 1) and $T$ burtoni (Clades $2+3$, combined).

For microsatellite data from sponges belonging to the $T$. burtoni clades, a principal coordinate analysis (PCA) based on genetic distances from allele frequencies was performed in the adegenet package (Jombart 2008) in R (https://www. r-project.org/). Assignment of individuals into genetic clusters was also assessed using STRUCTURE, which is a Bayesian clustering analysis for multilocus genotype data which probabilistically assigns individuals to clusters (Pritchard et al. 2000). Analyses were carried out in STRUCTURE v 2.3.2 (Pritchard et al. 2000), using 100,000 Markov Chain Monto Carlo (MCMC) iterations, a burn in of 10,000 iterations, 10 replicates per run and with the $K$ value set from 1 to 8 . The optimal $K$ was determined in Structure Harvester (Earl and vonHoldt 2012) to be $K=4$, and the 10 replications for $K=4$ were merged in CLUMPP (Jakobsson and Rosenberg 2007). Plots were visualized in MS Excel (2016). Clusters within T. burtoni were consistent with phylogenetic clades produced by $18 \mathrm{~S}$ and $\mathrm{rnl}$, and the degree of differentiation between these two groups was assessed using Fst. Because individuals belonging to Clade 3 contained loci that failed to amplify (missing data), these loci were removed for calculations of Fst. A pairwaise Fst value was calculated from the remaining 5 loci with significance determined by performing 20,000 permutations in Arlequin 3.1 (Excoffier et al. 2005). In addition, because measures of differentiation based on distance are sensitive to deviations from Hardy Weinberg Equilibrium (HWE; Waples 2015), Fst was calculated again removing one (out of five) loci that was not in HWE (Supplementary Information).

\section{Morphological analysis}

To determine if morphological characteristics were consistent with the observed genetic clades, a subsample of six sponges from each clade was selected for spicule analysis. We chose genetically identical individuals that fell consistently into the same clade for all three markers. Samples were collected from the same location and at the same time in order to reduce potential environmental and seasonal variation in spicule morphology (Bell et al. 2002). Each sponge was cut in half to photograph its cross section. Further, a small piece of tissue was embedded in paraffin wax and sectioned to $20 \mu \mathrm{m}$ using a rotary microtome (Leica Biosystems RM2235) to examine the location, arrangement and density of spicules across the ectosome and choanosome. A $\sim 5 \mathrm{~mm}^{3}$ tissue sample was then removed from each specimen and dissolved in bleach at $50{ }^{\circ} \mathrm{C}$ for $48 \mathrm{~h}$ and the remaining spicules were washed with distilled water and rinsed $3 \times$ with absolute ethanol, following the recommendations of Hooper (2003). One millilitre of the remaining spicule solution was mounted onto a slide using DPX and examined at a magnification of $\times 100-200$, using a compound light microscope (Leica Microsystems DM LB) with attached digital camera (Canon EOS 70D). Spicule type (see Supplementary Information for definitions of spicule types) and location (defined as region of tissue in the sponge-cortex versus choanosome) was recorded for a qualitative analysis. For a quantitative comparison, the sizes of 30 of each spicule type which were 


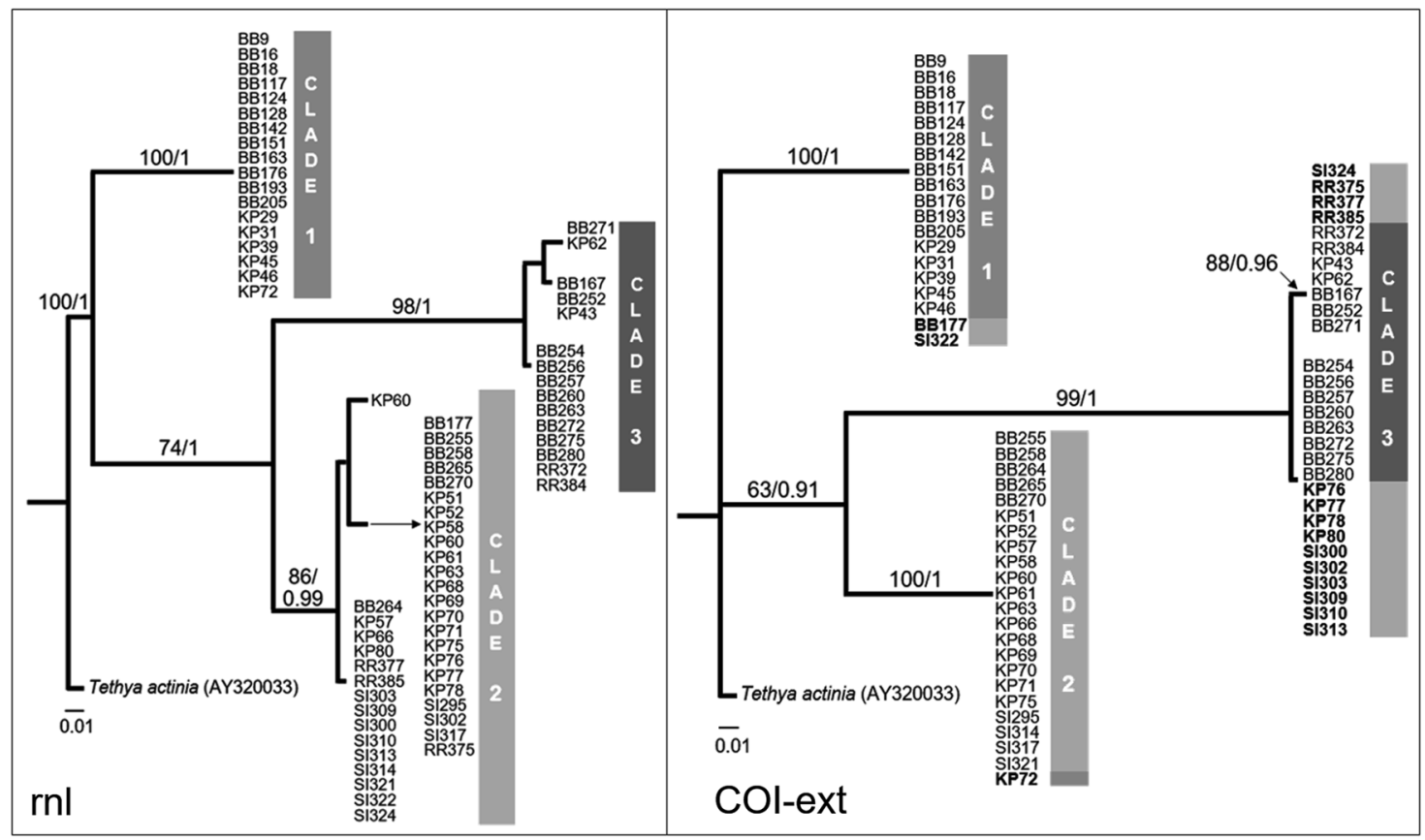

Fig. 2 Phylograms showing the relationship between Tethya bergquistae (Clade 1) and T. burtoni (Clades 2 and 3) based on individual mitochondrial markers (rnl and COI-ext). Topology generated from Maximum Likelihood (ML) analysis. ML bootstrap confidence values/ Bayesian Inference (BI) posterior probabilities given on each branch.
T. actinia was selected as an outgroup. Inconsistencies in the COI-ext tree versus $\mathrm{rnl}$ are highlighted by having sponges retain the colour of the rnl placement and by bolding individuals. Scale bar $=$ substitutions per site present in all sponges (strongyloxea, megasters and micrasters) were recorded per sponge. For megascleres (strongyloxea), the length and width were recorded for both thicker and thinner auxiliary types (stronglyoxea and styles, respectively). For both megasters and micrasters, the diameter and $\mathrm{R}: \mathrm{C}$ ratio (ray length to centre length ratio) were recorded, following Bergquist and Kelly-Borges (1991) and Sàra and Sàra (2004). Size measurements for all megasters (spherasters and oxyspherasters) were grouped, as well as micrasters (oxyasters, chiasters, stronglyasters and tylasters), because they could not be differentiated using light microscopy. Sizes were measured using ImageJ (Schneider et al. 2012). Non-parametric multidimensional scaling (nMDS) ordinations were generated using Euclidean distance on Primer v7 (Clarke 1993) to determine any clustering based on average, minimum and maximum sizes. Spicules from all 18 sponges that were subsampled for spicule analysis were also observed using Scanning Electron Microscopy (SEM), to visualize the megasters and micrasters at higher resolution and magnification. Spicules preserved in ethanol were mounted on stubs, air dried and coated with a gold/palladium alloy using a sputter coater. Scanning electron micrographs were taken with the Hitachi TM3000 Benchtop Scanning Electron Microscope in high vacuum mode.

\section{Results}

\section{Molecular phylogeny}

A total of $1420 \mathrm{bp}$ (425 bp for $18 \mathrm{~S}, 570 \mathrm{bp}$ for $\mathrm{rnl}$ and $425 \mathrm{bp}$ for COI-ext) were sequenced for 71 Tethya individuals. For all trees, branch confidence (via bootstrap/posterior probability values) ranged from 64 to $100 \% / 0.91$ to 1 for divergences between the main clades (Figs. 2 and 3). $18 \mathrm{~S}$ resolved the clades above $86 \% / 0.96$ and rnl above $74 \% / 0.99$, but COI-ext exhibited slightly lower confidence from $63 \% / 0.91$. The nuclear $18 \mathrm{~S}$ region grouped sponges into three genotypes, and for the mitochondrial rnl and COIext partitions there were seven and four haplotypes present, respectively. The concatenated data set for the mitochondrial markers revealed 15 different haplotypes (Fig. 3). For each individual locus, phylogenetic reconstructions revealed three divergent clades that each contained samples from all sampling locations; in other words, none of the clades contained individuals from strictly one location. For rnl and $18 \mathrm{~S}$, those sponges that were thought to be $T$. bergquistae all grouped together into one clade (Clade 1), but for COIext, one individual that was thought to be $T$. bergquistae grouped with Clade 2 (T. burtoni clade). For T. burtoni, rnl and $18 \mathrm{~S}$ generated congruent placement of sponges into two 


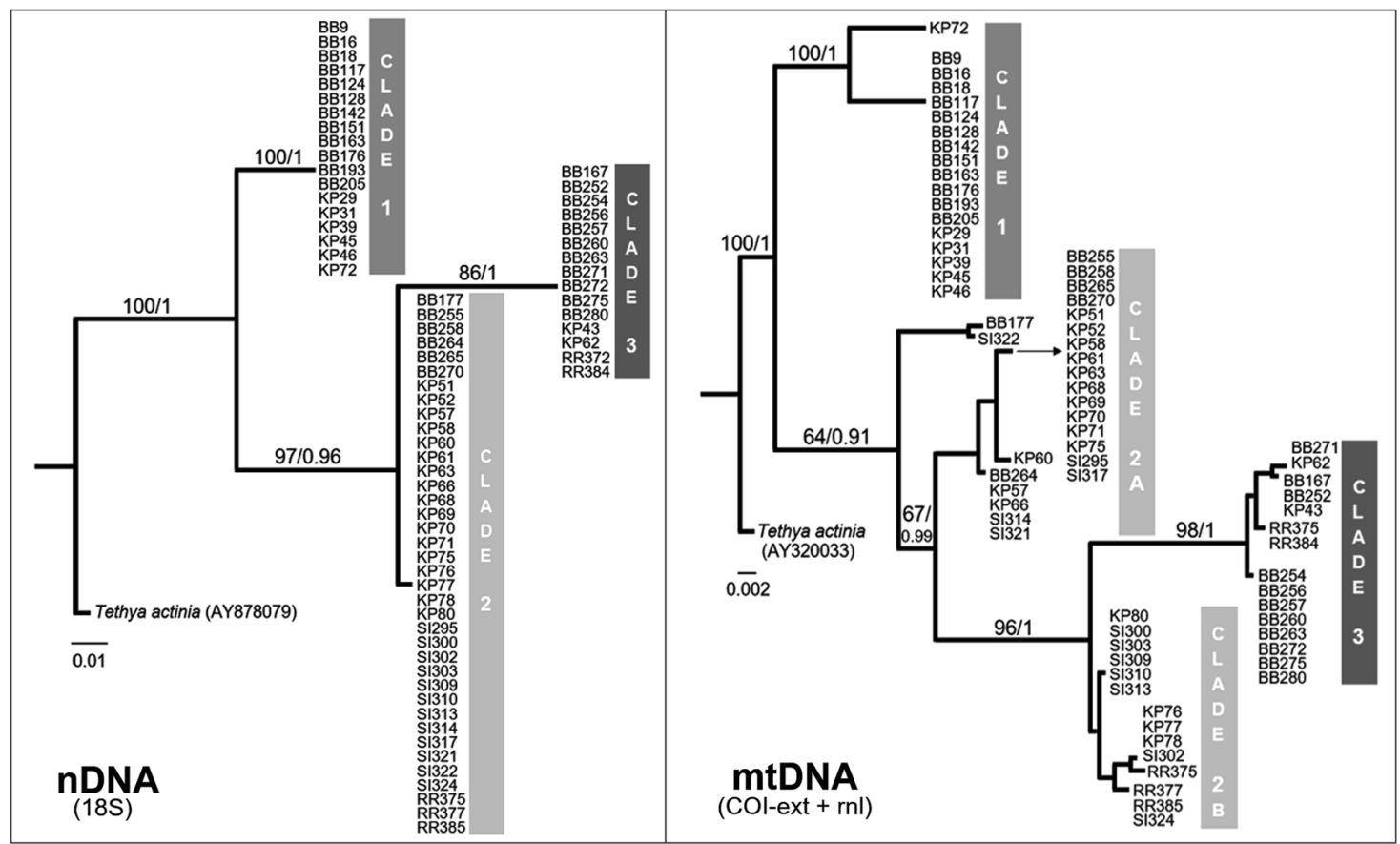

Fig. 3 Phylograms showing the relationship between Tethya bergquistae (Clade 1) and T. burtoni (Clades 2A $+\mathrm{B}$ and 3) based on nuclear DNA (18S) and mitochondrial DNA (concatenated COI-ext + rnl). Topology generated from Maximum Likelihood (ML) analysis.

ML bootstrap confidence values/Bayesian Inference (BI) posterior probabilities given on each branch. T. actinia was selected as an outgroup. Scale bar $=$ substitutions per site

separate groups. However, COI-ext placed sponges differently, where some sponges that belonged to Clade 2 for $\mathrm{rnl}$ and $18 \mathrm{~S}$ were placed into Clade 3; and in addition, two sponges which were thought to be $T$. burtoni were placed into Clade 1 ( $T$. bergquistae clade). The three uncertain yellow sponges (Fig. 1) grouped with $T$. bergquistae for all three markers.

Divergence was calculated between and within all genetic clades using $p$ distances (Table 2). Intraclade $p$ distance across all three markers ranged from 0 to $0.13 \%$ (average $=0.04 \%$, stdev $=0.06 \%$ ), whereas interclade $p$ distances for all pairwise clade comparisons across all markers ranged from 0.5 to $11.2 \%$ (average $=5.29 \%$, stdev $=3.83 \%$ ). For all pairwise clade comparisons, interclade $p$ distances for $18 \mathrm{~S}(0.5-1.2 \%)$ were lower than for the mtmarkers rnl and COI-ext (4.8-8.6, 6.4-11.2\%, respectively). For $T$. bergquistae (Clade 1), intraclade $p$ distances for all markers was zero. For T. burtoni (Clades $2+3$, combined), intraclade $p$ distances were: zero for $18 \mathrm{~S}, 2.1 \%$ for $\mathrm{rnl}$ and $4.2 \%$ for COI-ext. The divergence between $T$. bergquistae and T. burtoni was: $0.8 \%$ for $18 \mathrm{~S}, 6.6 \%$ for $\mathrm{rnl}$ and $9.0 \%$ for COI-ext.

For T. burtoni, both PCA and STRUCTURE based on microsatellite allele frequencies revealed two distinct genetic clusters that were congruent with the $T$. burtoni clades generated from phylogenetic analyses for $18 \mathrm{~S}$ and $\mathrm{rnl}$ (Fig. 4). The T. burtoni clades (Clades 2 and 3) were

Table 2 Genetic divergence ( $p$ distances, \%) for Tethya spp. for all gene markers, where Clade 1 is $T$. bergquistae and Clades 2 and 3 are T. burtoni. Intraclade $p$ distances are reported for individuals within clades, while interclade values are pairwise comparisons between all clades.

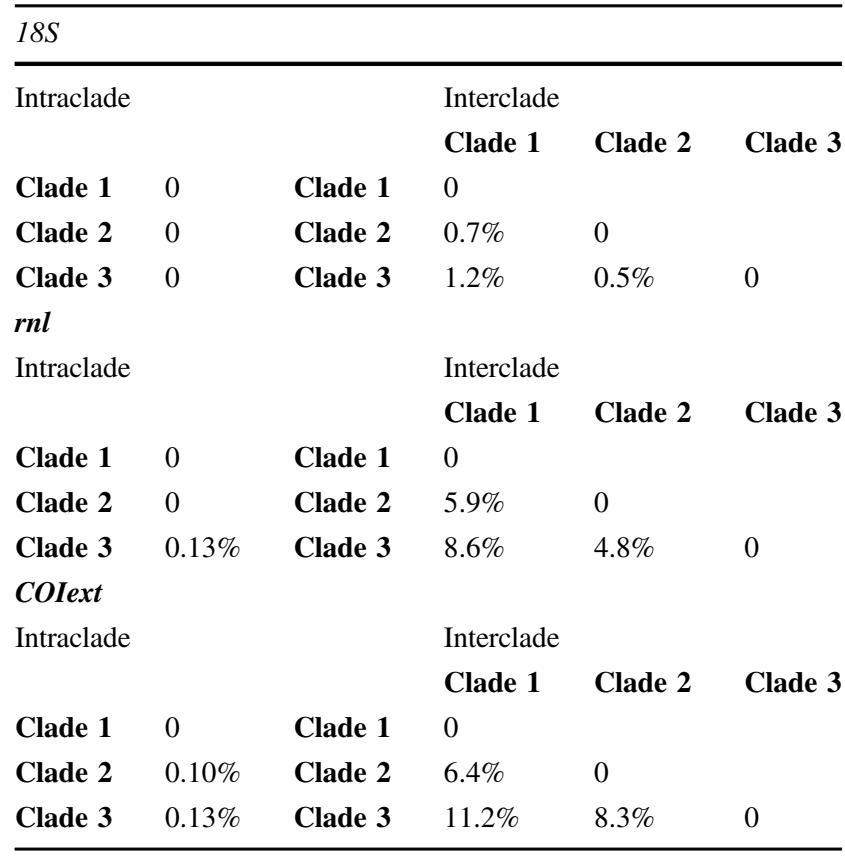

significantly different with respect to their genetic structure (Fst $=0.39, P<0.0001)$. When using only those loci in 


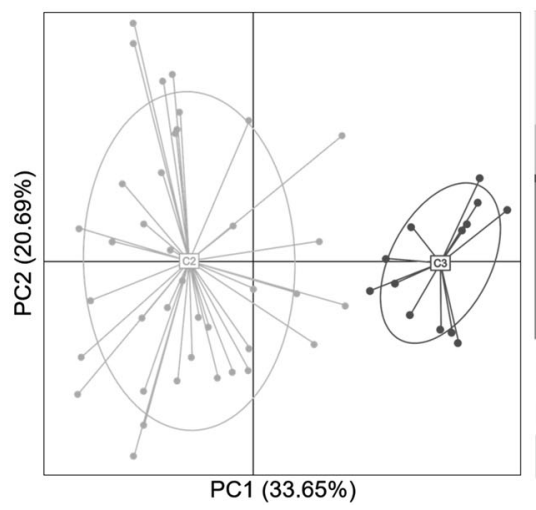

Fig. 4 Genetic differentiation within Tethya burtoni based on microsatellite data. On left: Principal coordinate analysis (PCA) based on genetic distances from microsatellite allele frequencies for the $T$. burtoni clades (Clades 2 and 3 from Fig. 3). Each point represents a $T$. burtoni genotype, and the two groups ( $\mathrm{C} 2$ and $\mathrm{C} 3)$ are labelled inside of their $67 \%$ inertia ellipses. On right: Group assignment of $T$. burtoni individuals into four genetic clusters $(K=4)$ based on STRUCTURE.
Each vertical line represents an individual, and the coloration is the proportion of that individual's estimated membership into each of the four genetic clusters. Labels along the horizontal refer to individual IDs (see Supplementary Table S1 for more information). Horizontal bars under individual IDs correspond to sampling locations, which are: Kapiti Island (black), Red Rocks (dark grey), Breaker Bay (light grey) and Somes Island (white)

Table 3 Mean lengths for spicules in Tethya bergquistae (Clade 1) and T. burtoni (Clades $2+3$ ) for three genetic clades identified by $18 \mathrm{~S}$ and rnl (Figs. 2 and 3) and for all samples combined (overall). Strongyloxea are presented as length $\times$ width. R:C refers to ray length:diameter-to-centre ratios. All sizes are in microns $(\mu \mathrm{m})$

\begin{tabular}{|c|c|c|c|c|c|c|}
\hline & $\begin{array}{l}\text { Stronglyoxea }(l \times w) \\
(\mu \mathrm{m})\end{array}$ & $\begin{array}{l}\text { Style }(l \times w) \\
(\mu \mathrm{m})\end{array}$ & $\begin{array}{l}\text { Megaster diameter } \\
(\mu \mathrm{m})\end{array}$ & Megaster R:C & $\begin{array}{l}\text { Micraster diameter } \\
(\mu \mathrm{m})\end{array}$ & Micraster R:C \\
\hline Clade 1 & $\begin{array}{l}1032.0 \pm 219.4 \times 22.7 \pm \\
5.7\end{array}$ & $\begin{array}{l}356.4 \pm 103.6 \times 14.4 \pm \\
3.3\end{array}$ & $42.9 \pm 12.5$ & $0.75 \pm 0.15$ & $13.7 \pm 3.2$ & $0.81 \pm 0.19$ \\
\hline Clade 2 & $\begin{array}{l}1054.7 \pm 320.4 \times 21.6 \pm \\
4.6\end{array}$ & $\begin{array}{l}424.1 \pm 108.1 \times 13.6 \pm \\
3.8\end{array}$ & $44.7 \pm 6.4$ & $0.73 \pm 0.15$ & $15.2 \pm 1.4$ & $0.79 \pm 0.16$ \\
\hline Clade 3 & $\begin{array}{l}1104.0 \pm 273.5 \times 16.7 \pm \\
5.3\end{array}$ & $\begin{array}{l}419.7 \pm 124.7 \times 9.9 \pm \\
2.9\end{array}$ & $44.1 \pm 9.0$ & $0.86 \pm 0.15$ & $12.4 \pm 2.0$ & $1.07 \pm 0.25$ \\
\hline Overall & $\begin{array}{l}1061.2 \pm 275.3 \times 20.6 \pm \\
5.8\end{array}$ & $\begin{array}{l}399.0 \pm 115.9 \times 12.8 \pm \\
3.9\end{array}$ & $43.9 \pm 9.7$ & $0.77 \pm 0.16$ & $13.8 \pm 2.6$ & $0.88 \pm 0.23$ \\
\hline
\end{tabular}

HWE, differentiation between clades was also significant and strong $(\mathrm{Fst}=0.47, P<0.0001)$.

\section{Morphological analysis}

All sponges contained megascleres that were strongyloxea and anisostronglyes, along with smaller, thinner auxiliary styles. Megasters were spherasters and oxyspherasters, and micrasters were tylasters, chiasters, stronglyasters and oxyasters. Spicule sizes are summarized in Table 3. Average strongyloxea and auxiliary style length $\times$ width were $1061 \pm 275 \times 21 \pm 6 \mu \mathrm{m}$ and $399 \pm 116 \times 13 \pm 4 \mu \mathrm{m}$, respectively. Respective megaster and micraster diameters were $44 \pm 10$ and $14 \pm 3 \mu \mathrm{m}$, and the average $\mathrm{R}: \mathrm{C}$ ratio was $\sim 0.8 \pm 0.2$ for all asters. n-MDS plots for spicule size (average, minimum and maximum sizes) revealed no evident clustering (Fig. 5). Qualitative differences were observed between the location and density of megasters within the different genetic clades. Those sponges belonging to Clade 1 contained megasters throughout both the cortex and choanosome, whereas those sponges in Clades 2 and 3 had megasters in the cortex, but little to none in the choanosome. Within the T. burtoni clades (Clades 2 and 3), there were no differences in spicule composition detectable by light microscopy. Scanning electron micrographs of spicules from the three genetic clades also revealed no visible differences in megastar or micraster morphology (Fig. 6). However, sponges belonging to the T. bergquistae clade contained an additional spined oxyaster, which was absent in the T. burtoni clades. A qualitative assessment of cross sections from this subsample of sponges revealed high plasticity within each genetic clade, and no clear differences between clades (Fig. 1). Further, $20 \mu \mathrm{m}$ cross sections examining the spicule composition between the choanosome and ectosome revealed that $T$. bergquistae contained more asters in the choanosome than T. burtoni (Fig. 7). 
Fig. 5 Nonparametricmultidimensional (nMDS) scaling ordination of mean spicule sizes for Tethya bergquistae (pink squares, Clade 1 ) and $T$. burtoni (orange circles, Clade 2; and blue triangles, Clade 3). Clades are identified in Fig. 3. Spicules sizes are based on measurements from strongyloxea, megasters and micrasters ( $n=30$ per sponge). Codes above each sample refer to individual sample ID (refer to Supplementary Table S1 for more information on individual samples). A subset of all samples are presented in the nMDS, based on a Euclidean distance matrix

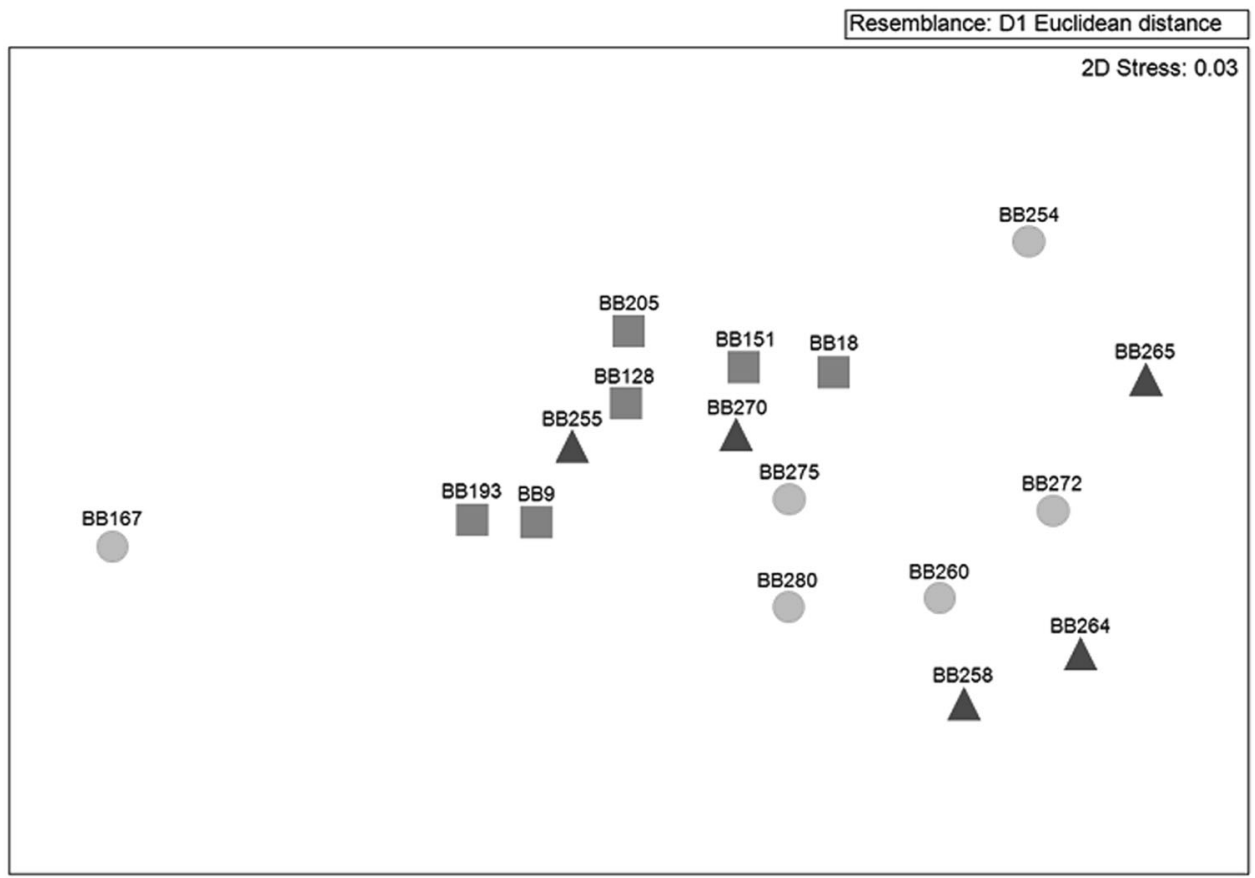

However, within $T$. burtoni, there were no evident differences between Clades 2 and 3. While some members of Clade 2 appeared to contain a slightly higher density of asters in the cortex, this was not consistent for all individuals within that clade.

\section{Discussion}

Here, we document discordance between molecular and morphological descriptions of Tethya spp. in central New Zealand and highlight the potential for cryptic speciation to go undetected based on morphological features alone. Phylogenetic reconstructions based on 18S, rnl and COI-ext revealed three genetic clades, with a speciation event likely occurring in the group described as T. burtoni. This division within $T$. burtoni was further confirmed using novel microsatellite markers. Morphological analyses based on spicule size and composition allowed $T$. bergquistae to be differentiated from $T$. burtoni but failed to reveal differences between the $T$. burtoni clades. To our knowledge, this is only the second report of cryptic species on the basis of molecular differences within the group Tethya (with the first being T. robusta, Sarà et al. 1993). Understanding how organisms evolve is central to ecology and evolution, but can be particularly complex for phenotypically similar organisms living in sympatry with no obvious barriers to reproduction, reflected in Tethya spp. examined here. Overall, our study highlights the need to use more than one method to define sponge species boundaries in a reliable way.

\section{Species delineation based on genetics}

The phylogenetic relationship of Tethya spp. was not congruent for all three markers, as COI-ext placed sponges into different clades with most discrepancies occurring in the $T$. burtoni group. Microsatellite markers also supported the $T$. burtoni clade division that was indicated in the $18 \mathrm{~S}$ and $\mathrm{rnl}$ trees (Figs. 2 and 3). The use of COI to delineate species has had differential success among sponges (Erpenbeck et al. 2006b; Wörheide and Erpenbeck 2007; Erpenbeck et al. 2007; Poppe et al. 2010; Belinky et al. 2012) as well as other organisms (Shearer and Coffroth 2008; Derycke et al. 2010), resulting in debate around its use as an informative marker (Waugh 2007). The COI region often has low variability (Bucklin et al. 2011), and this conservativeness can fail to capture evolutionary relationships between closely related species. We instead employed COI-ext to avoid this drawback, as this region downstream of $\mathrm{COI}$ is thought to substitute earlier during species divergence (Erpenbeck et al. 2006a). We captured variation between $T$. bergquistae and $T$. burtoni, as well as within $T$. burtoni; however, the relationship produced from COI-ext was incongruent with that produced from $18 \mathrm{~S}$, rnl and the 11 microsatellite markers. For species living in sympatry, introgression can be common, resulting in semipermeable species boundaries (Rüber et al. 2001, Harrison and Larson 2014). Genes can introgress at different rates (called differential introgression), which can result in phylogenies from multiple markers in disagreement (Harrison and Larson 2014). It is possible that the inconsistency in the COI-ext phylogeny is a product of this, and if true, gives evidence of introgression 

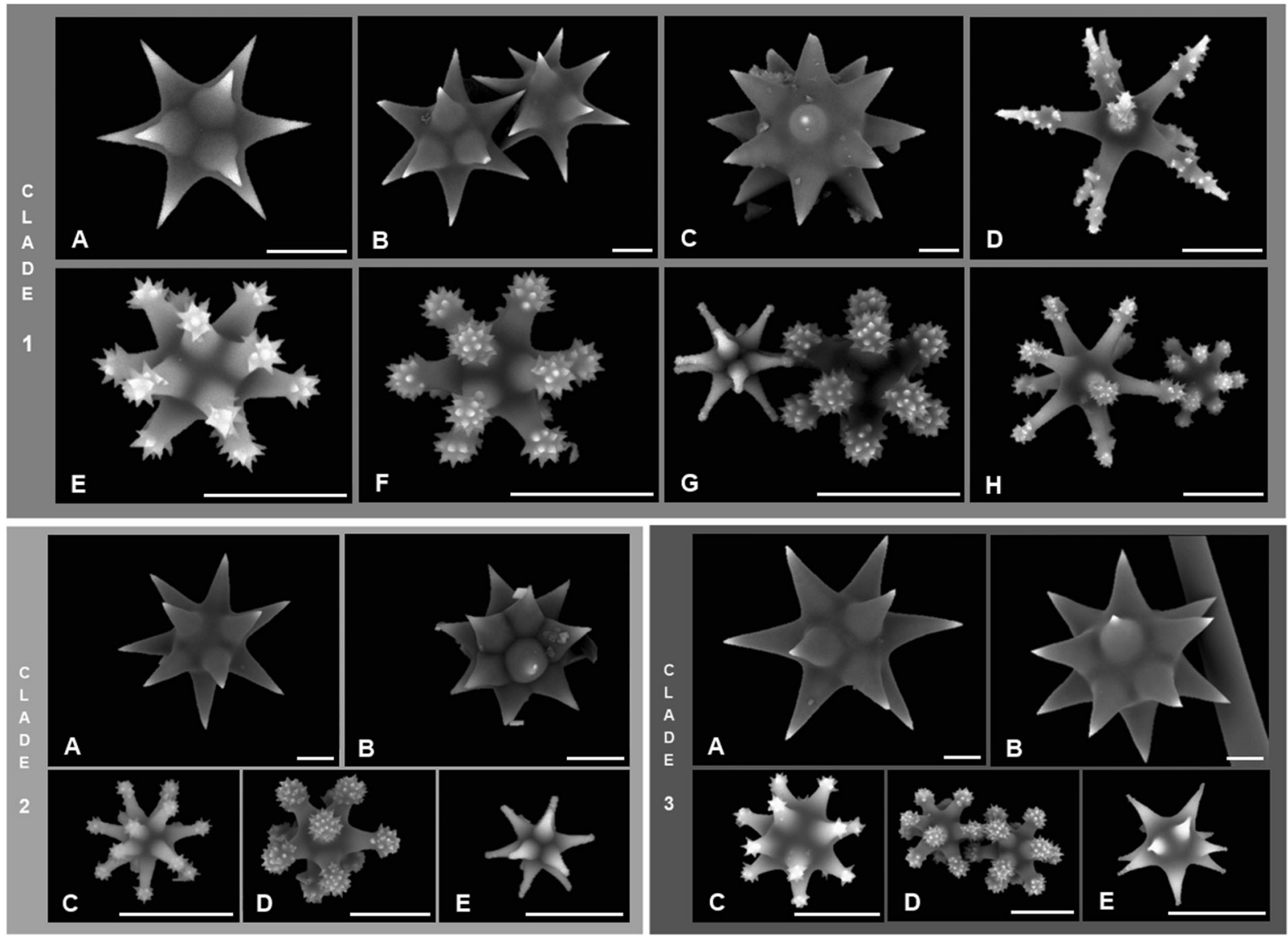

Fig. 6 Scanning electron micrographs of spicules belonging to Tethya bergquistae (Clade 1) and T. burtoni (Clade 2+3), from sponges belonging to the three genetic clades of Tethya identified in Fig. 3. Clade 1: $\mathrm{A}-\mathrm{C}=$ spherasters; $\mathrm{D}=$ spined oxyaster; $\mathrm{E}-\mathrm{H}=$ tylasters; $\mathrm{G}$

$=$ oxyaster. Clade 2 and 3: $\mathrm{A}-\mathrm{B}=$ spherasters; $\mathrm{C}-\mathrm{D}=$ tylasters; $\mathrm{E}=$ oxyaster. Scale bars are located in the bottom right of each micrograph and represent $10 \mu \mathrm{m}$

between $T$. bergquistae and T. burtoni. For instance, one sponge (KP72) that was thought to be $T$. bergquistae (as evidenced by its placement into Clade 1 for $18 \mathrm{~S}$ and $\mathrm{rnl}$ ) was placed into the T. burtoni clade (Clade 2) for COI-ext. In addition, two sponges (BB177 and SI322), which were thought to be $T$. burtoni (from their placement into Clade 2 by $18 \mathrm{~S}$ and $\mathrm{rnl}$ ), were placed into the $T$. bergquistae clade (Clade 1) for COI-ext. Within T. burtoni, there is more disagreement between COI-ext and the other markers within the clades. For example, those sponges belonging to Clade 2B of the mitochondrial concatenated data set (Fig. 3) were placed into Clade 2 by $18 \mathrm{~S}$, rnl and the microsatellite markers, but Clade 3 for COI-ext. It is possible that this group of sponges, which possess genes common to two different putative species (discussed below), have also undergone introgressive hybridization. Some corals have also been found to exhibit high degrees of sympatry, cryptic speciation and introgression (Ladner and Palumbi 2012), and perhaps shared life-history traits between corals and sponges (i.e. sessile, sexual and asexual reproductive ecology) shape the evolutionary history of these populations.

While additional sequencing work would provide clarity on this topic, it appears highly probable that the species boundaries between $T$. bergquistae and T. burtoni, as well as between the putative $T$. burtoni cryptic species, are actually semipermeable.

The definition of a species continues to be debated, and when complex evolutionary processes like introgression can shape species, understanding species delineations becomes more complicated. The topology produced from $18 \mathrm{~S}$ clearly differentiates our clades. $18 \mathrm{~S}$ is a slowly evolving gene region (Berntson et al. 2001), and as such often captures interspecific variation rather than intraspecific variation. Three separate clades, despite the slow evolving nature of this gene, provides strong evidence that these three clades correspond to three different species. Most interestingly, two clades exist within $T$. burtoni and suggest cryptic speciation within the group. Other measures exist (e.g. $p$ distances, Fst) to more definitively set a quantitative value to define species divisions. Comparing the $p$ distances obtained in this study to other observed divergence values for sponges (Table 4), both within- and between-clade 
Fig. 7 Cross sections of a small piece of tissue $(20 \mu \mathrm{m}$ thick) from Tethya spp., where Clade 1 is $T$. bergquistae and Clades 2 and 3 are T. burtoni. Arrows are pointing to the line that divides the outer cortex and the inner choanosomal tissue. Black scale bars represent $500 \mu \mathrm{m}$
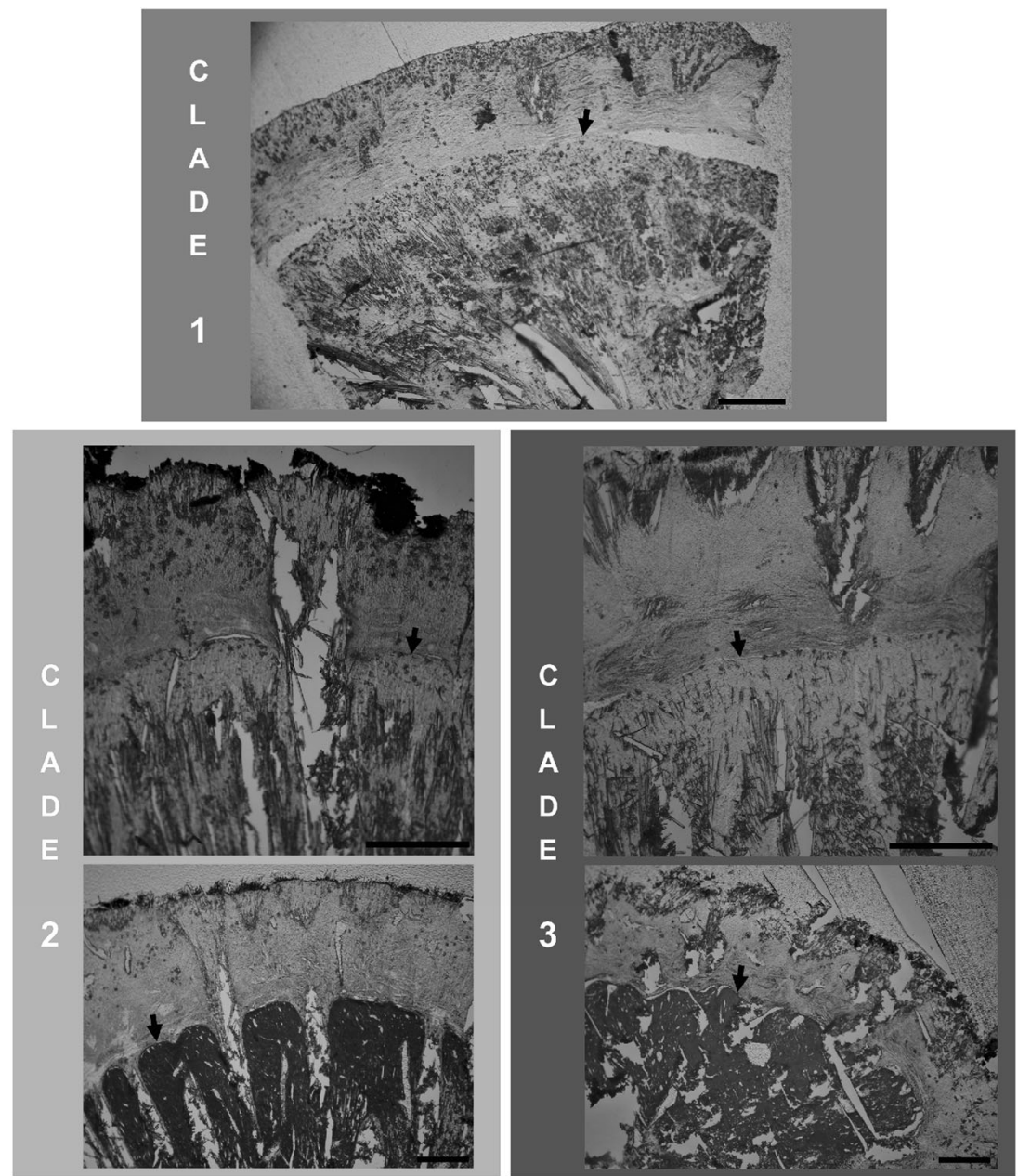

distances give further evidence for species-level relatedness. Our intraclade $p$ distances were about $~ 5-50$ times less than the interclade values for all pairwise comparisons of clades, which is consistent with intraspecific values for other sponge taxa. These intraclade distances suggest that sponges belonging to the same clade are conspecific. When comparing the individual markers used here to the same markers (or similarly evolving markers) in other studies, the interclade $p$ distances calculated for all pairwise clade comparisons in this study compare to interclade $p$ distances for other sponge species (Table 4). This further reveals our clades do in fact correspond to species. For example, Scopalina lophyropoda was reported as being comprised of a cryptic species complex (Blanquer and Uriz 2007), and the interclade $p$ distances for $18 \mathrm{~S}(0.3-2 \%)$ were on the same scale as for those clades we found within $T$. burtoni $(0.5 \%)$. Considering $T$. bergquistae (Clade 1) versus $T$. burtoni (Clades $2+3$, combined), divergence between the two species was: $0.8 \%$ for $18 \mathrm{~S}, 6.6 \%$ for $\mathrm{rnl}$ and $9 \%$ for COIext. Divergence between the T. burtoni clades (Clade 2 versus Clade 3 ) were on the same order: $0.5 \%$ for $18 \mathrm{~S}, 4.8 \%$ for $\mathrm{rnl}$ and $8.3 \%$ for COI-ext. Divergence within T. burtoni that is on the same scale as between $T$. burtoni and $T$. bergquistae gives further evidence that the two T. burtoni clades correspond to different species. The microsatellite data also reveal strong differentiation within the $T$. burtoni group (Fst $=0.39, P<0001)$. While microsatellite markers are traditionally not used as a tool for phylogenetic studies (but instead population structure analysis), used here in combination with the other genetic markers gives further evidence for strong genetic differentiation between clades. Interestingly, differentiation between both $T$. burtoni clades was not a product of location (population structure of $T$. burtoni discussed elsewhere), as evident from the STRUCTURE plot (Fig. 4). Instead, sponges that lived sympatrically were strongly genetically different, suggesting reproductive isolation for sponges in the same location. Cryptic speciation has been recorded throughout the Porifera phylum (Solé-Cava et al. 1991; Muricy et al. 1996; Nichols and Barnes 2005; Wörheide et al. 2008; Xavier et al. 2010; DeBiasse and Hellberg 2015; Knapp et al. 2015; Uriz et al. 2017), and is also likely for what's known as $T$. burtoni. We believe our genetic data combined gives strong evidence that $T$. burtoni is in fact be comprised of a species 
Table 4 Summary of genetic divergence ( $p$ distances, \%) for various sponge species. Ranges given for intraclade and interclade $p$ distances

\begin{tabular}{|c|c|c|c|c|}
\hline Species & Marker & $\begin{array}{l}\text { Intraclade } \\
p \text { distance }\end{array}$ & $\begin{array}{l}\text { Interclade } \\
p \text { distance }\end{array}$ & Reference \\
\hline \multirow[t]{2}{*}{ Aplysina spp. } & $\mathrm{COI}$ & 0 & - & \multirow{2}{*}{$\begin{array}{l}\text { Cruz-Barraza } \\
\text { et al. } 2012\end{array}$} \\
\hline & $\begin{array}{l}\text { ITS1- } \\
5.8- \\
\text { ITS2 }\end{array}$ & 0 & $0.1-2.2$ & \\
\hline $\begin{array}{l}\text { Astrosclera } \\
\text { willeyana }\end{array}$ & $\mathrm{COI}$ & - & $0.2-0.4$ & $\begin{array}{l}\text { Wörheide } \\
2006\end{array}$ \\
\hline \multirow[t]{2}{*}{ Cliona spp. } & $\mathrm{COI}$ & - & $2.7-8.7$ & \multirow{2}{*}{$\begin{array}{l}\text { De Paula et al } \\
2012\end{array}$} \\
\hline & ITS & - & $5.9-14.8$ & \\
\hline \multirow[t]{2}{*}{ Cliona celata $^{\mathrm{a}}$} & COI & - & $2.7-8.3$ & \\
\hline & ITS & - & $8.1-11.6$ & \\
\hline \multirow[t]{3}{*}{ Cliona celata $^{\mathrm{a}}$} & COI & $0.1-0.5$ & $6.2-8.4$ & \multirow{3}{*}{$\begin{array}{l}\text { Xavier et al. } \\
2010\end{array}$} \\
\hline & Atp8 & $0-0.5$ & $7.9-16$ & \\
\hline & $28 \mathrm{~S}$ & 0 & $2.8-5.4$ & \\
\hline \multirow[t]{2}{*}{ Haliclona sp. } & ITS1 & $1.7-3.0$ & - & \multirow{2}{*}{$\begin{array}{l}\text { Redmond and } \\
\text { McCormack } \\
2009\end{array}$} \\
\hline & ITS2 & $0.7-1.8$ & - & \\
\hline \multirow{3}{*}{$\begin{array}{l}\text { Hemimycale } \\
\text { spp. }\end{array}$} & $\mathrm{COI}$ & - & $8.4-8.7$ & \multirow{3}{*}{$\begin{array}{l}\text { Uriz et al. } \\
2017\end{array}$} \\
\hline & $28 \mathrm{~S}$ & - & $1.8-2.2$ & \\
\hline & $18 \mathrm{~S}$ & - & $1.4-1.9$ & \\
\hline \multirow[t]{2}{*}{ Hexadella spp. } & $\mathrm{COI}$ & $0-3.5$ & $3.9-8.7$ & \multirow{2}{*}{$\begin{array}{l}\text { Reveillaud } \\
\text { et al. } 2010\end{array}$} \\
\hline & ATPS & $0-6.3$ & $10-28.5$ & \\
\hline Ianthella basta ${ }^{\mathrm{a}}$ & COI & - & $0.5-1.75$ & $\begin{array}{l}\text { Andreakis } \\
\text { et al. } 2012\end{array}$ \\
\hline \multirow{2}{*}{$\begin{array}{l}\text { Plocamionida } \\
\text { spp. }\end{array}$} & $\mathrm{COI}$ & 0.5 & $1.3-20$ & \multirow{2}{*}{$\begin{array}{l}\text { Reveillaud } \\
\text { et al. } 2011\end{array}$} \\
\hline & $28 \mathrm{~S}$ & - & $0.1-3.3$ & \\
\hline \multirow{3}{*}{$\begin{array}{l}\text { Scopalina } \\
\text { lophyropoda }\end{array}$} & COI & $0-3$ & $13-22$ & \multirow{3}{*}{$\begin{array}{l}\text { Blanquer and } \\
\text { Uriz } 2007\end{array}$} \\
\hline & $28 \mathrm{~S}$ & $0-0.8$ & $2-19$ & \\
\hline & $18 \mathrm{~S}$ & 0.1 & $0.3-2$ & \\
\hline \multirow[t]{3}{*}{ Tethya spp. } & $\begin{array}{l}\text { COI- } \\
\text { ext }\end{array}$ & $0-0.1$ & $6.4-11.2$ & \multirow[t]{6}{*}{ This study } \\
\hline & $\mathrm{rnl}$ & $0-0.1$ & $4.8-8.6$ & \\
\hline & $18 \mathrm{~S}$ & 0 & $0.5-1.2$ & \\
\hline \multirow[t]{3}{*}{ Tethya burtoni ${ }^{\mathrm{a}}$} & $\begin{array}{l}\text { COI- } \\
\text { ext }\end{array}$ & 0.1 & 8.3 & \\
\hline & $\mathrm{rnl}$ & $0-0.1$ & 4.8 & \\
\hline & $18 \mathrm{~S}$ & 0 & 0.5 & \\
\hline
\end{tabular}

${ }^{a}$ Reported as cryptic species

complex, being more genetically diverse than previously thought based on morphology alone.

\section{Discordance between molecular and morphological characteristics}

Our analysis of spicule size/type, in conjunction with our qualitative assessment of macromorphological features (colour and cross sections), was inconsistent with the molecular phylogeny that we generated. Macromorphological features allowed sponges belonging to the $T$. bergquistae clade to be distinguished from sponges belonging to the T. burtoni clades; however, we were not able to identify sponges belonging to separate genetic clades within $T$. burtoni from morphology. The three uncertain, bright yellow specimens grouped together with those sponges belonging to $T$. bergquistae, which has not previously been described as yellow (Bergquist and KellyBorges 1991; Hooper and Wiedenmayer 1994; Battershill et al. 2010), indicating that colour is not a reliable distinguishing feature in this case. Qualitative observations revealed no obvious differences in cross sections between $T$. burtoni clades, and instead were highly variable within each clade. While generally cross sections are a key defining feature between species of Tethya, they did not provide clear resolution here. There was a marked difference between the spicule composition of T. bergquistae and the $T$. burtoni complex (more asters in the choanosome of the former), but there were no differences evident within the $T$. burtoni clades. Morphological characteristics therefore provided no clear evidence of speciation within T. burtoni. Because there is a lack of defining characteristics available for Porifera, it is common for genetic diversity (and cryptic speciation) to go undetected, where molecular and morphological relationships are commonly incongruent (McCormack et al. 2002; Erpenbeck et al. 2006b; Xavier et al. 2010). Determining which morphological traits are informative in species differentiation can also be complicated. For example, Erpenbeck et al. (2006b) documented that sponges containing highly divergent skeletal features are actually closely genetically related. In contrast, spicules within the same species may be highly variable due to environmental influences (Bell et al. 2002). We show here that morphological features may not be able to reveal species boundaries, as perhaps skeletal changes following divergence may be delayed. Mutations may accumulate without altering the phenotype when nucleotide changes have no effect on how its product (protein) folds and its function overall (Avise 2012), which could explain delayed morphological expression in genetically different individuals. While it is possible that subtle morphological differences may exist, they were not able to be detected in this study. The high phenotypic plasticity observed across individuals of $T$. burtoni resulted in a lack of clear diagnostic features for sponges belonging the different genetic clades. Furthermore, the potential introgression events between cryptic species may make detecting morphological differences between such species more difficult. To avoid this potential confounding factor, we used sponges which showed no introgression based on our markers for the 
morphological analysis presented here. However, we cannot confirm that these sponges have not undergone introgression, as we only surveyed three genes. A survey of other regions of the genome may reveal clearer relationships and show the scope of introgressive hybridization between the T. burtoni cryptic species. This may shed more light on differences between plasticity versus distinct morphological features that allow species to be characterized. With such incongruence between morphological and molecular data, it is imperative to use a combination of methods to correctly delineate species in order to fully understand the species evolutionary relationships.

\section{Conclusions}

Our study highlights the complexity in delineating sympatric, morphologically similar species when disagreement between morphological and molecular traits exist, and the importance of using multiple taxonomic methods. While traditional morphology-based taxonomy can be the first step in species identification, we show that it can fail to reveal cryptic species. Being able to confidently identify a species is crucial to any ecological or evolutionary study, yet this remains a challenge. Organisms with few defining traits, like sponges, present an opportunity to examine potential morphological and molecular discord, and highlight the complexity in defining species boundaries. Failure to detect cryptic species may result in an underestimation of biodiversity and an incorrect interpretation of functional roles of an organism. In addition, it can mislead our understanding of connectivity patterns and evolutionary relationships, if interspecific diversity is confused for intraspecific variation. As such, we strongly recommend the use of integrative taxonomy in species identification.

Acknowledgements This research was funded by a Victoria University of Wellington Doctoral Scholarship awarded to Megan Shaffer. A special thanks to Dr. Michael Gardner and Tessa Bradford (Flinders University) for their help with microsatellite development, and to Daniel Leduc (National Institute of Water and Atmospheric Research) for training and use of the SEM. We are also grateful to Dr. Manuel Maldonado (Center for Advanced Studies of Blanes, CEAB-CSIC), who offered insight into the interpretation of our results. Lastly, thanks to the technicians at the Victoria University Coastal Ecology Lab (Daniel McNaughtan, John van der Sman, Dan Crossett) and to Bucky Focht, who aided in the collection of these samples.

\section{Compliance with ethical standards}

Conflict of interest The authors declare that they have no conflict of interest.

Data availability Sequence data have been submitted to GenBank, under the accession numbers MH180010-180023. Microsatellite genotype data is available on Pangaea (https://doi.org/10.1594/ PANGAEA.889125).

\section{References}

Adlard RD, Lester JG (1995) Development of a diagnostic test for Mikrocytos roughleyi, the aetiological agent of Australian winter mortality of the commercial rock oyster, Saccostrea commercialis (Iredale \& Roughley). J Fish Dis 18:609-614

Alvarez B, Crisp MD, Driver F, Hooper JNA, Van Soest RWM (2000) Phylogenetic relationships of the family Axinellidae (Porifera: Demospongiae) using morphological and molecular data. Zool Scr 29:169-198

Andreakis N, Luter HM, Webster NS (2012) Cryptic speciation and phylogeographic relationships in the elephant ear sponge Ianthella basta (Porifera, Ianthellidae) from northern Australia. Zool J Linn Soc 166:225-235

Avise JC (2012) Molecular markers, natural history and evolution. Springer Science \& Business Media, New York, NY

Battershill CN, Bergquist PR, Cook SDC (2010) Phylum Porifera. In: Cook SDC (ed) New Zealand coastal marine invertebrates, vol 1. Canterbury University Press, Christchurch, New Zealand, pp 122-124.

Baum DA, Shaw KL (1995) Genealogical pers pectives on the species problem. In: Hoch PC, Stevenson AG (eds) Experimental and molecular approaches to plant biosystematics, vol 53. Missouri Botanical Garden, St. Louis, pp 289-303

Bavestrello G, Bonito M, Sará M (1993) Influence on depth on the size of sponge spicules. Sci Mar 57:415-420

Belinky F, Szitenberg A, Goldfarb I, Feldstein T, Wörheide G, Ilan M et al. (2012) ALG11 - a new variable DNA marker for sponge phylogeny: Comparison of phylogenetic performances with $18 \mathrm{~S}$ rDNA and the COI gene. Mol Phylogenet Evol 63:702-713

Bell J, Barnes D, Turner J (2002) The importance of micro and macro morphological variation in the adaptation of a sublittoral demosponge to current extremes. Mar Biol 140:75-81

Bergquist PR (1961) Demospongiae (Porifera) of the Chatham Islands and Chatham Rise, collected by the Chatham Islands 1954 Expedition. NZ Oceanogr Inst Mem 13:169-206

Bergquist PR, Kelly-Borges M (1991) An evaluation of the genus Tethya (Porifera: Demospongiae: Hadromerida) with descriptions of new species from the southwest Pacific. Beagle, Rec North Territ Mus Arts Sci 8:37-72

Berntson EA, Bayer FM, McArthur AG, France SC (2001) Phylogenetic relationships within the Octocorallia (Cnidaria: Anthozoa) based on nuclear 18S rRNA sequences. Mar Biol 138:235-246

Bickford D, Lohman DJ, Sodhi NS, Ng PL, Meier R, Winker K et al. (2007) Cryptic species as a window on diversity and conservation. Trends Ecol Evol 22:148-155

Blanquer A, Uriz MJ (2007) Cryptic speciation in marine sponges evidenced by mitochondrial and nuclear genes: a phylogenetic approach. Mol Phylogenetics Evol 45:392-397

Bond C (1992) Continuous cell movements rearrange anatomical structures in intact sponges. J Exp Zool A Ecol Integr Physiol 263:284-302

Boore JL (1999) Animal mitochondrial genomes. Nucleic Acids Res 27:1767-1780

Bucklin A, Steinke D, Blanco-Bercial L (2011) DNA barcoding of marine metazoan. Ann Rev Mar Sci 3:471-508

Capa M, Pons J, Hutchings P (2013) Cryptic diversity, intraspecific phonetic plasticity and recent geographical translocations in Branchiomma (Sabellidae, Annelida). Zool Scr 42:637-655

Clarke KR (1993) Non-parametric multivariate analyses of changes in community structure. Aust J Ecol 18:117-143 
Corriero G, Gadaleta F, Bavestrello G (2015) A new Mediterranean species of Tethya (Porifera: Tethyida: Demospongiae). Ital J Zool 82:535-543

Cruz-Barraza JA, Carballo JL, Rocha-Olivares A, Ehrlich H, Hog M (2012) Integrative taxonomy and molecular phylogeny of genus Aplysina (Demospongiae: Verongida) from Mexican Pacific PLoS ONE 7:e42049

Czarna A, Gawrońska B, Nowińska R, Morozowska M, Kosiński P (2016) Morphological and molecular variation in selected species of Erysimum (Brassicaceae) from Central Europe and their taxonomic significance. Flora 222:68-85

De Paula TS, Zilberberg C, Haidu E, Lobo-Haidu G (2012) Morphology and molecules on opposite sides of the diversity gradient: four cryptic species of the Cliona celata (Porifera, Demospongiae) complex in South America revealed by mitochondrial and nuclear markers. Mol Phylogenet Evol 62:529-541

De Queiroz K (2007) Species concepts and species delimitation. Syst Biol 56:879-886

DeBiasse MB, Hellberg ME (2015) Discordance between morphological and molecular species boundaries among Caribbean species of the reef sponge Callyspongia. Ecol Evol 5:663-675

Derycke S, Vanaverbeke J, Rigaux A, Backeljau T, Moens T (2010) Exploring the use of cytochrome oxidase c subnit 1 (COI) for DNA barcoding of free-living marine nematodes. PLoS ONE 5: e13716. https://doi.org/10.1371/journal.pone.0013716

Earl DA, vonHoldt BM (2012) STRUCTURE HARVESTER: a website and program for visualizing STRUCTURE output and implementing the Evanno method. Conserv Genet Resour 4:359-361

Erpenbeck D, Hooper JNA, Wörheide G (2006a) CO1 phylogenies in diploblasts and the 'Barcoding of Life' - are we sequencing a suboptimal partition? Mol Ecol Notes 6:550-553.

Erpenbeck D, Breeuwer JAJ, Parra-Velandia FJ, Van Soest RWM (2006b) Speculation with speculation? Three independent gene fragments and biochemical characters versus morphology in demosponge higher classification. Mol Phylogenet Evol 38:293-205.

Erpenbeck D, Duran S, Rutzler K, Paul V, Hooper JNA, Wörheide G (2007) Towards a DNA taxonomy of Caribbean demosponges: a gene tree reconstructed from partial mitochondrial CO1 gene sequences supports previous rDNA phylogenies and provides a new perspective on the systematics of Demospongiae. J Mar Biol Assoc UK 87:1563-1570

Erwin PM, Thacker RW (2007) Phylogenetic analyses of marine sponges within the order Verongida: a comparison of morphological and molecular data. Invertebr Biol 126:220-234

Excoffier L, Laval G, Schneider S (2005) Arlequin ver. 3.0: an integrated software package for population genetics data analysis. Evol Bioinform Online 1:47-50

Folmer O, Black M, Hoeh W, Lutz R, Vrijenhoek R (1994) DNA primers for amplification of mitochondrial cytochrome $\mathrm{C}$ oxidase subunit I from diverse metazoan invertebrates. Mol Mar Biol Biotechnol 3:294-299

Frankham R, Ballou JD, Dudash MR, Eldridge MDB, Fenster CB, Lacy RC et al. (2012) Implications of different species concepts for conserving biodiversity. Biol Conserv 153:25-31

Gómez A, Serra M, Carvalho GR, Lunt DH (2002) Speciation in ancient cryptic species complexes: evidence from the molecular phylogeny of Branchionus plicatilis (Rotifera). Evolution 56:1431-1444

Harrison RG, Larson EL (2014) Hybridization, introgression and the nature of species boundaries. J Hered 105:795-809

Hebet PDN, Penton EH, Burns JM, Janzen DH, Hallwachs W (2004) Ten species in one: DNA barcoding reveals cryptic species in the neotropical skipper butterfly Astraptes fulgerator. Proc Natl Acad Sci USA 41:14812-14817
Heim I, Nickel M, Brümmer F (2007) Phylogeny of the genus Tethya (Tethyidae: Hadromerida: Porifera): molecular and morphological aspects. J Mar Biol Assoc UK 87:1615-1627

Hennig W (1966) Phylogenetic systematics. University of Illinois Press, Urbana

Hey J, Waples RS, Arnold ML, Butlin RK, Harrison RG (2003) Understanding and confronting species uncertainty in biology and conservation. Trends Ecol Evol 18:597-603

Hilis DM (1987) Molecular versus morphological approaches to systematics. Annu Rev Ecol Evol Syst 18:23-42

Hooper JNA (2003) 'Sponguide'. A guide to sponge collection and identification. In: Hooper JNA, Van Soest RWM (eds) 2002. Systema Porifera. A guide to the classification of sponges, vol 1-2. Kluwer Academic/Plenum Publishers, New York, NY, Boston, Dordrecht, London, Moscow, pp xlviii-1708.

Hooper JNA, Wiedenmayer F (1994) Porifera. In: Wells A (ed) Zoological catalogue of Australia, vol 12. CSIRO, Melbourne, pp $1-620$.

Hooper JNA, Van Soest RWM (2002) Systema Porifera. a guide to the classification of sponges. Kluwer Academic/Plenum Publishers, New York, NY, Boston, Dordrecht, London, Moscow

Hulce D, Li X, Snyder-Leiby T (2011) GeneMarker genotyping software: tools to increase the statistical power of DNA fragment analysis. J Biomol Tech 22:S35-S36

Jakobsson M, Rosenberg NA (2007) CLUMPP: a clustering matching and permutation program for dealing with label switching and multimodality in analysis of population structure. Bioinformatics 23:1801-1806

Jombart T (2008) Adegenet: R package for the multivariate analysis of genetic markers. Bioinformatics 24:1403-1405

Kauserud H, Svegarden IB, Decock C, Hallenberg N (2006) Hybridization among cryptic species of the cellar fungus Coniophora puteana (Basidiomycota). Mol Ecol 16:389-399

Kearse M, Moir R, Wilson A, Stones-Havas S, Cheung M, Sturrock S et al. (2012) Geneious basic: an integrated and extendable desktop software platform for the organization and analysis of sequence data. Bioinformatics 28:1647-1649

Knapp IS, Forsman ZH, Williams GJ, Toonen RJ, Bell JJ (2015) Cryptic species obscure introduction pathway of the blue Carribean sponge (Haliclona (Soestella) caerulea), (order: Haplosclerida) to Palmyra- Atoll, Central Pacific. Peer J 3: e1170

Knowlton N (1993) Sibling species in the sea. Annu Rev Ecol Syst 24:189-216

Knowlton N (2000) Molecular genetic analyses of species boundaries in the sea. Hydrobiologia 420:73-90

Kumar S, Stecher G, Tamura K (2015) MEGA7: molecular evolutionary genetics analysis version 7.0 for bigger datasets. Mol Biol Evol 33:1870-1874

Ladner JT, Palumbi SR (2012) Extensive sympatry, cryptic diversity and introgression throughout the geographic distribution of two coral species complexes. Mol Ecol 21:2224-2238

Larkin MA, Blackshields G, Brown NP, Chenna R, McGettigan PA, McWilliam H et al. (2007) ClustalW and ClustalX. Bioinformatics 23:2947-2948

Lavrov DV, Wang XJ, Kelly M (2008) Reconstructing ordinal relationships in the Demospongiae using mitochondrial genomic data. Mol Phylogenet Evol 49:111-124

Lecompte E, Denys C, Granjon L (2005) Confrontation of morphological and molecular data: the Praomys group (Rodentia, Murinae) as a case of adaptive convergences and morphological stasis. Mol Phylogenet Evol 37:899-919

Ledenfield R (1888) Descriptive catalogue of sponges in the Australian Museum, Sydney. Taylor and Francis, London

Lihová J, Kučera J, Perný M, Marhold K (2007) Hybridization between two polypoloid Cardamine (Brassicacea) species in 
north-western Spain: Discordance between morphological and genetic variation patterns. Ann Bot 99:1083-1096

Mayden RL (1997) A hierarchy of species concepts: the denouement in the saga of the species problem. In: Claridge MF, Dawah HA, Wilson MR (eds) Species: the units of biodiversity. Chapman and Hall, London, pp 381-424.

Mayr E (1942) Systematics and the origin of species. Columbia University Press, New York, NY

McCormack GP, Erpenbeck D, Van Soest RWM (2002) Major discrepancy between phylogenetic hypotheses based on molecular and morphological criteria within the Order Haplosclerida (Phylum Porfera: Class Demospongiae). J Zool Syst Evol Res 40:237-240

McDonald JI, Hooper JNA, McGuinness KA (2002) Environmentally influenced variability in the morphology of Cinachyrella australiensis (Carter 1886) (Porifera: Spirophorida: Tellidae). Mar Freshw Res 53:79-84

Morrow C, Cárdenas P (2015) Proposal for a revised classification of the Demospongiae (Porifera). Front Zool 12:7

Muricy G, Solé-Cava AM, Thorpe JP, Boury-Esnault N (1996) Genetic evidence for extensive cryptic speciation in the subtidal sponge Plakina trilopha (Porifera: Demospongiae: Homoscleromorpha) from the Western Mediterranean. Mar Ecol Prog Ser 138:181-187

Nakayama S, Arima K, Kawai K, Mohri K, Inui C, Sugano W et al. (2015) Dynamic transport and cementation of skeletal elements build up the pole-and-beam structured skeleton of sponges. Curr Biol 25:2549-2554

Nichols SA, Barnes PAG (2005) A molecular phylogeny and historical biogeography of the marine sponge genus Placospongia (Phylum Porifera) indicate low dispersal capabilities and widespread crypsis. J Exp Mar Biol Ecol 323:1-15

Nylander JAA (2004) MrModeltest v2. Program distributed by the author. Evolutionary Biology Centre, Uppsala University: Sweden.

Padial JM, Miralles A, De la Rive I, Vences M (2010) The integrative future of taxonomy. Front Zool 7:16

Poppe J, Sutcliffe P, Hooper JNA, Wörheide G, Erpenbeck D (2010) COI barcoding reveals new clades and radiation patterns of IndoPacific sponges of the family Irciniidae (Demospongiae: Dictyoceratida). PLoS ONE 5:e9950

Plotkin A, Voight O, Willassen E, Rapp HT (2017) Molecular phylogenies challenge the classification of Polymastiidae (Porifera, Demospongiae) based on morphology. Org Divers Evol 17:45-66

Pritchard K, Ward V, Battershill C, Bergquist PR (1984) Marine sponges: forty-six sponges of Northern New Zealand. Leigh Lab Bull 14:149

Pritchard JK, Stephens M, Donnelly P (2000) Inference of population structure using multilocus genotype data. Genetics 155:945-959

Redmond NE, McCormack GP (2009) Ribosomal internal transcriber spacer regions are not suitable for intra- or inter-specific phylogeny reconstruction in haplosclerid sponges (Porifera: Demospongiae). J Mar Biol Assoc UK 89:1251-1256

Reveillaud J, Remerie T, Van Soest R, Erpenbeck D, Cardenas P, Derycke $S$ et al. (2010) Species boundaries and phylogenetic relationships between Altanto-Mediterranean shallow-water and deep-sea coral associated with Hexadella species (Porifera, Ianthellidae). Mol Phylogenet Evol 56:104-114

Reveillaud J, van Soest R, Derycke S, Picton B, Rigaux A, Vanreusel A (2011) Phylogenetic relationships among NE Atlantic Plocamionida Topsent (1927) (Porifera, Poecilosclerida): underestimated diversity in reef ecosystems. PLoS ONE 6:e16533

Ronquist F, Teslenko M, Van der Mark P, Ayres D, Darling A, Höhna $S$ et al. (2012) MrBayes 3.2: efficient Bayesian phylogenetic inference and model choice across a large model space. Syst Biol 61:539-542

Rosen DE (1979) Fishes from the uplands and intermontane basins of Guatemala: revisionary studies and comparative geography. Bull Am Mus Nat Hist 162:267-376

Rot C, Goldfarb I, Ilan M, Huchon D (2006) Putative cross-kingdom horizontal gene transfer in sponge (Porifera) mitochondria. BMC Evol Biol 6:71

Rüber L, Meyer A, Sturmbauer C, Verheyen E (2001) Population structure in two sympatric species of the Lake Tanganyika cichlid tribe Eretmodini: evidence for introgression. Mol Ecol 10:1207-1225

Sarà M (1987) A study on the genus Tethya (Porifera Demospongiae) and new perspectives in sponge systematics. In: Vacelet J, BouryEsnault N (eds) Taxonomy of Porifera. NATO ASI Series (Series G: Ecological Sciences), vol 13. Springer, Berlin.

Sarà M (1998) A biogeographic and evolutionary survey of the genus Tethya (Porifera, Demospongiae). In: Watanabe Y, Fusetani N (eds) Sponge sciences, vol 1. Springer, Tokyo, pp 83-94.

Sarà M, Corriero G, Bavestrello G (1993) Tethya (Porifera, Demospongiae) species coexisting in a Maldivian coral reef lagoon: Taxonomical, genetic and ecological data. Mar Ecol 14:341-355

Sarà M, Sarà A (2004) A revision of Australian and New Zealand Tethya (Porifera: Demospongiae) with preliminary analysis of species-groupings. Invertebr Syst 18:117-156

Schneider CA, Rasband WS, Eliceiri KW (2012) NIH Image to ImageJ: 25 years of image analysis. Nat Methods 9:671-675

Shearer TL, Coffroth MA (2008) DNA barcoding: barcoding corals: limited by interspecific divergence, not intraspecific variation. Mol Ecol Res 8:247-255

Simpson GG (1951) The species concept. Evolution 5:285-298

Solé-Cava AM, Klautau M, Boury-Esnault N, Borojecic R, Thorpe JP (1991) Genetic evidence for cryptic speciation in allopatric populations of two cosmopolitan species of the calcareous sponge genus Clathrina. Mar Biol 111:381-386

Swofford DL (2002) PAUP*. Phylogenetic analysis using parsimony (*and other methods). Version 4. Sinaeur Associates, Sunderland, Massachusetts

Uriz MJ, Garate L, Agell G (2017) Molecular phylogenies confirm the presence of two cryptic Hemimycale species in the Mediterranean and reveal the polyphyly of the genera Crella and Hemimycale (Demospongiar: Poecilosclerida). Peer J 5:e2958

Van Soest RWM, Boury-Esnault N, Hooper JNA, Rützler K, de Voogd NJ, Alvarez B, et al. (2018) World Porifera database. Accessed at http://www.marinespecies.org/porifera/porifera.php? $\mathrm{p}=$ taxdetails\&id $=132077$ on $2017-10-14$

Van Valen L (1976) Ecological species, multispecies, and oaks. Taxon $25: 233-239$

Vrijenhoek RC (2009) Cryptic species, phenotypic plasticity, and complex life histories: assessing deep-sea faunal diversity with molecular markers. Deep-Sea Res Part II 56:1713-1723

Wallis GP, Cameron-Christie SR, Kennedy HL, Palmer G, Sanders TR, Winter DJ (2017) Interspecific hybridization causes longterm phylogenetic discordance between nuclear and mitochondrial genomes in freshwater fishes. Mol Ecol 26:3116-3127

Waples RS (2015) Testing for Hardy-Weinberg proportions: have we lost the plot? J Hered 106:1-19

Waugh J (2007) DNA barcoding in animal species: progress, potential and pitfalls. Phylogenet Syst 29:188-197

Wendel JF, Doyle JJ (1998) Phylogenetic incongruence: window into genome history and molecular evolution. In: Soltis DE, Soltis PS, Doyle JJ (eds) Molecular systematic of plants II. Springer, Boston

Wörheide G, Erpenbeck D (2007) DNA taxonomy of sponges progress and perspectives. J Mar Biol Assoc UK 87:1629-1633

Wörheide G, Epp LS, Macis L (2008). Deep genetic divergence among Indo-Pacific populations of the coral reef sponge Leucetta 
chagoensis (Leucettidae): founder effects, vicariance, or both? BMC Evol Biol 8: https://doi.org/10.1186/1471-2148 $-8-24$.

Xavier JR, Rachello-Dolmen PG, Parra-Velandia F, Schönberg CHL, Breeuwer JAJ, van Soest RWM (2010) Molecular evidence of cryptic speciation in the "cosmopolitan" excavating sponge
Cliona celata (Porifera, Clionaidae). Mol Phylogenet Evol $56: 13-20$

Wörheide G (2006) Low variation in partial cytochrome oxidase subunit I (COI) mitochondrial sequences in the coralline demosponge Astrosclera willeyana across the Indo-Pacific. Mar Biol 148:907-912 